Studien zum Physik- und Chemielernen H. Niedderer, H. Fischler, E. Sumfleth [Hrsg.]

260

Frank Finkenberg

\title{
Flipped Classroom im Physikunterricht
}




\section{Studien zum Physik- und Chemielernen}

Herausgegeben von Hans Niedderer, Helmut Fischler und Elke Sumfleth

Diese Reihe im Logos-Verlag bietet ein Forum zur Veröffentlichung von wissenschaftlichen Studien zum Physik- und Chemielernen. In ihr werden Ergebnisse empirischer Untersuchungen zum Physik- und Chemielernen dargestellt, z. B. über Schülervorstellungen, Lehr-/Lernprozesse in Schule und Hochschule oder Evaluationsstudien. Von Bedeutung sind auch Arbeiten über Motivation und Einstellungen sowie Interessensgebiete im Physik- und Chemieunterricht. Die Reihe fühlt sich damit der Tradition der empirisch orientierten Forschung in den Fachdidaktiken verpflichtet. Die Herausgeber hoffen, durch die Herausgabe von Studien hoher Qualität einen Beitrag zur weiteren Stabilisierung der physik- und chemiedidaktischen Forschung und zur Förderung eines an den Ergebnissen fachdidaktischer Forschung orientierten Unterrichts in den beiden Fächern zu leisten. 
Studien zum Physik- und Chemielernen

Band 260 

Frank Finkenberg

Flipped Classroom im Physikunterricht 
Studien zum Physik- und Chemielernen

Hans Niedderer, Helmut Fischler, Elke Sumfleth [Hrsg.]

Bibliografische Information der Deutschen Nationalbibliothek

Die Deutsche Nationalbibliothek verzeichnet diese Publikation in der Deutschen Nationalbibliografie; detaillierte bibliografische Daten sind im Internet über http://dnb.d-nb.de abrufbar.

(C) Copyright Logos Verlag Berlin GmbH 2018

Alle Rechte vorbehalten.

ISBN 978-3-8325-4737-0

ISSN 1614-8967

(C) $(1) \Theta$

Logos Verlag Berlin GmbH

Comeniushof, Gubener Str. 47,

D-10243 Berlin

Tel.: $\quad+49(0) 30 / 42851090$

Fax: $+49(0) 30 / 42851092$

https://www.logos-verlag.de 
Eingereicht am: 23.04 .2018

bei der Fakultät für Physik und Astronomie

1. Gutachter: Prof. Dr. Thomas Trefzger, Universität Würzburg

2. Gutachter: Prof. Dr. Roger Erb, Universität Frankfurt der Dissertation

Vorsitzende(r): Prof. Dr. Ansgar Denner, Universität Würzburg

1. Prüfer: $\quad$ Prof. Dr. Thomas Trefzger, Universität Würzburg

2. Prüfer: $\quad$ Prof. Dr. Roger Erb, Universität Frankfurt

3. Prüfer: $\quad$ Prof. Dr. Karl Mannheim, Universität Würzburg im Promotionskolloquium

Tag des Promotionskolloquiums: 27.06.2018 


\section{Zusammenfassung}

In der Unterrichtsmethode Flipped Classroom sind schulische und häusliche Aktivitäten vertauscht. Instruktionale Elemente werden in online verfügbare Lernvideos ausgelagert, welche die Schüler als häusliche Vorbereitung ansehen. Im Unterricht stehen dann schülerzentrierte Tätigkeiten im Vordergrund, in denen die Schüler ihr Wissen anwenden und vertiefen können. Durch die Auslagerung von Inputphasen wandelt sich die Rolle des Lehrers vom Instructor zum Lernbegleiter.

Die vorliegende quasi-experimentelle Studie im Pre-/Postdesign mit Kontrollgruppe untersuchte die Wirkungen des Flipped Classroom in Physikkursen der Oberstufe (Grundkursniveau) an zwei deutschen Gymnasien mit $N=151$ Schülerinnen und Schülern. Acht Physikkurse der 11. Jahrgangsstufe nahmen an der Studie teil, die sich über einen Zeitraum von zwei Schuljahren erstreckte (2015/16 und 2016/17). Vier der fünf teilnehmenden Lehrkräfte unterrichteten sowohl einen Kontroll- als auch einen Treatmentkurs. Sämtliche Lernvideos wurden von den Lehrkräften selbst erstellt. Dabei integrierten sie reale Experimente, um dem Anspruch physikauthentischen Unterrichts gerecht zu werden. Die Forschungsfragen richteten sich sowohl auf die Leistung in einem Fachwissenstest als auch auf affektive Lernmerkmale wie die Motivation, das Interesse und das Selbstkonzept. Zusätzlich wurden die wahrgenommene Lehrerunterstützung und das Hausaufgabenverhalten untersucht.

Die Anwendung von Flipped Classroom im Physikunterricht zeigte größtenteils positive Effekte. Die Schülerinnen und Schüler im Flipped Classroom hatten einen höheren kognitiven Lernzuwachs und ein besseres Selbstkonzept als ihre Mitschüler, die traditionell unterrichtet wurden. Das Leistungsniveau und das Geschlecht der Schülerinnen und Schüler hatten dabei keinen Einfluss auf diese Effekte. Während die Motivation, sich mit Physik zu beschäftigen, in der Kontrollgruppe sank, blieb sie in der Treatmentgruppe auf konstantem Niveau. Bei genauerem Blick zeigte sich, dass die Motivation bei Schülerinnen im Flipped Classroom anstieg, bei Schülerinnen im traditionellen Unterricht jedoch abnahm. Das Interesse am Unterrichtsfach Physik wurde in beiden Gruppen geringer. Sowohl die wahrgenommene Lehrerunterstützung als auch die Hausaufgabendauer blieben in beiden Gruppen zwischen Pre- und Posttest unverändert. Die Hausaufgabendisziplin war im Flipped Classroom jedoch deutlich höher, was zeigt, dass die Schülerinnen und Schüler eher bereit waren, sich instruktionale Lernvideos anzusehen als klassische Hausaufgaben zu bearbeiten. 


\begin{abstract}
Flipped Classroom inverts traditional teaching methods by delivering direct instruction in online learning videos. The students watch the videos at home so that class time is freed up for student centered and collaborative activities that allow a deeper exploration of the content. By outsourcing lectures, the role of the teacher shifts from instructing to coaching the students.

The quasi-experimental pre/post-study with control group examined the effects of flipped classroom applied to basic physics courses at two German secondary schools with $\mathrm{N}=151$ students in a three-months-treatment. Eight $11^{\text {th }}$ grade physics courses took part in the study that was conducted in the school years 2015/16 and 2016/17. Four of five teachers involved in the study taught both control and treatment courses. All videos were produced by the teachers and incorporated real experiments to ensure an authentic physics education experience. The research questions focused on the performance in a content knowledge test as well as noncognitive attitudes such as motivation, interest and self-concept. In addition, perceived teacher support and homework habits were also evaluated.

Applying flipped classroom in physics school education showed largely positive results. The students in flipped classroom had a higher gain in cognitive learning and a better selfconcept than those in a traditional classroom setting. Physics aptitude as well as gender did not moderate these effects. Whereas the motivation to engage in physics declined in the control group, it remained unchanged in the treatment group. In particular, female students in flipped classroom developed a higher motivation to engage in physics than their female peers who lost motivation in the traditional classroom. The interest in physics as a school subject decreased in both groups. The perceived teacher support and the average length of homework stayed the same in both groups between pre- and post-test. However, the homework discipline was considerably higher in flipped classroom which showed that students were more likely to watch instructional videos than do traditional homework.
\end{abstract}




\section{Inhaltsverzeichnis}

\section{LERNEN UND FLIPPED CLASSROOM - EINE THEORETISCHE FUNDIERUNG}

2.1 Schüleraktivierung und erfolgreiches Lernen

2.2 Motivation, Interesse, Selbstkonzept und Lernleistung 6

2.2.1 Motivation in der Selbstbestimmungstheorie 6

2.2.2 Interesse: individuell, situational, Sach- und Fachinteresse $\quad 8$

2.2.3 Selbstkonzept: schulisch und fachspezifisch 11

2.2.4 Schulische Lernleistung 13

2.3 Flipped Classroom als Unterrichtsmethode $\quad 15$

2.3.1 Ursprung und Formen des Flipped Classroom 15

2.3.2 Gemeinsamer Nenner: Basisform des Flipped Classroom 17

2.3.3 Theoretische Ebenen: Schüleraktivierung, Selbstbestimmung, Lernbegleitung und multimediales Lernen $\quad 19$

2.3.4 Probleme im Flipped Classroom 22

2.4 Flipped Classroom in der didaktischen Forschung 22

2.4.1 Bisherige Forschungsergebnisse zum Flipped Classroom 23

2.4.2 Physikdidaktisches Interesse am Flipped Classroom 26

3 FORSCHUNGSFRAGEN UND HYPOTHESEN

\section{INHALTE UND LERNMATERIALIEN}

4.1 Inhalte der Unterrichtsreihe »Elektromagnetische Induktion und elektromagnetischer Schwingkreis «

4.2 Pool an Übungsaufgaben 


\subsection{Untersuchungsdesign}

5.1.1 Schulorganisatorischer Rahmen 44

5.1.2 Teilnehmende Lehrkräfte 44

5.1.3 Dauer und Ablauf der Untersuchung 45

5.2 Beschreibung des traditionellen Unterrichts $\quad 46$

$\begin{array}{lll}\text { 5.3 Beschreibung der Intervention } & 48\end{array}$

$\mathbf{5 . 4}$ Messinstrumente $\quad \mathbf{5 1}$

5.4.1 Fachwissenstest $\quad 51$

5.4.2 Fragebogen 54

$\begin{array}{lll}5.5 & \text { Auswertungsmethoden } & 58\end{array}$

6 ERGEBNISSE UND DISKUSSIONEN

6.1 Ausgangslage: Deskriptive Statistik zur Stichprobe $\quad 61$

$\begin{array}{lll}\text { 6.1.1 Kognitive Lernausgangslage } & 62\end{array}$

6.1.2 Affektive Lernausgangslage und Stützfaktoren 64

6.2 Forschungsfrage 1: Einfluss auf Lernleistung, Motivation, Interesse und Selbstkonzept

$\begin{array}{lll}\text { 6.2.1 Hypothese 1a: Lernleistung } & 67\end{array}$

6.2.2 Hypothese 1b: Motivation, Fachinteresse und Selbstkonzept 68

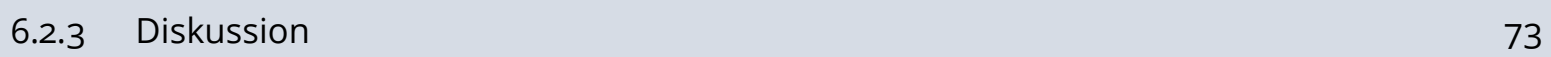

6.3 Forschungsfrage 2: Einfluss von Leistungsniveau und Geschlecht 77

6.3.1 Hypothese 2a: Leistungsniveau $\quad 78$

6.3.2 Hypothese 2b: Geschlechtereffekt $\quad 80$

6.3.3 Einfluss der Lehrkraft $\quad 86$

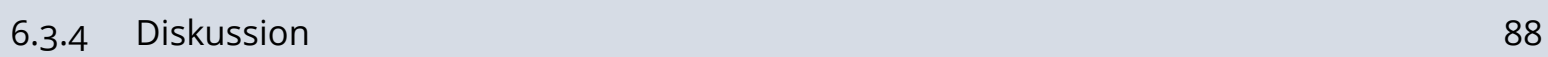

6.4 Forschungsfrage 3: Veränderung der Lehrerunterstützung und der Hausaufgabenbearbeitung $\quad 90$

6.4.1 Hypothese 3a: Lehrerunterstützung 90

6.4.2 Hypothese 3b: Hausaufgabendisziplin und -dauer 91

6.4.3 Einfluss der Lehrkraft $\quad 95$

$\begin{array}{lll}6.4 .4 & \text { Diskussion } & 97\end{array}$ 
6.5 Rückmeldungen der Schüler im Flipped Classroom 99

6.5.1 Lernvideos und häusliche Vorbereitungszeit 101

6.5.2 Übungsaufgaben im Unterricht und Schüleraktivierung 104

$\begin{array}{ll}\text { 6.5.3 Experimente im Physikunterricht } & 105\end{array}$

$\begin{array}{lll}6.5 .4 & \text { Veränderte Lehrerrolle } & 106\end{array}$

6.5.5 Weitere Rückmeldungen 106

$\begin{array}{ll}\text { 6.5.6 Diskussion } & 107\end{array}$

7 ZUSAMMENFASSUNG UND AUSBLICK 110

$\begin{array}{lll}\text { 7.1 Ergebnisse in zehn Punkten } & 110\end{array}$

$\begin{array}{lll}7.2 & \text { Kritische Betrachtung der Untersuchung } & 114\end{array}$

$\begin{array}{lll}7.3 & \text { Perspektiven } & 115\end{array}$

8 TABELLENVERZEICHNIS $\quad 119$

9 ABBILDUNGSVERZEICHNIS

10 LITERATURVERZEICHNIS 123

$\begin{array}{llr}11 & \text { ANHANG } & 139\end{array}$ 



\section{Einleitung}

Neue Mittel schaffen neue Wege, neue Medien schaffen neue Methoden. Die Transformation der Lebenswelt durch die Allgegenwart internetfähiger Endgeräte und digitaler Medien schließt auch die Lernwelt von Jugendlichen in Schulen ein. Auch wenn der Schulbereich üblicherweise nicht die Pionierarbeit in der Nutzung neuer Technologien leistet, so werden in den Schulen digitale Hilfsmittel eingesetzt, um bestehende Lehrmedien zu ersetzen, zu erweitern, zu ändern oder von Grund auf neu zu schaffen. Diese Implementierungsformen werden durch das SAMR-Modell (Substitution, Augmentation, Modification, Redesign) von Puentedura (2006) beschrieben. Der Mehrwert der Nutzung digitaler Medien in der schulischen Bildung geht über den inhaltlichen und motivationalen Aspekt hinaus und entspricht auch dem bildungspolitischen Auftrag zur Förderung der digitalen Kompetenz der Schüler. ${ }^{1}$

Flipped Classroom ist eine junge Unterrichtsmethode, die sich auf online verfügbare Lernvideos stützt, welche der individuellen Unterrichtsvorbereitung dienen. Im Unterricht steht dadurch mehr Zeit für schülerzentrierte Tätigkeiten zur Verfügung. Nach den Kriterien von Meyer (2002) spricht man hier von einer methodischen Großform. Sie hat sich parallel zum Ausbau leistungsfähiger Breitbandnetze entwickelt, die die infrastrukturellen Voraussetzungen für die Onlinenutzung von Lernvideos bieten. Verbreitung hat Flipped Classroom seit etwa 2010 zunächst vor allem in den USA und im englischen Sprachraum gefunden. In Deutschland, sowie auf dem übrigen europäischen Festland, wurde diese Methode später und dort zunächst im Hochschulbereich rezipiert, bis seit etwa 2014 der Einsatz vor allem im schulischen Mathematikunterricht nachweislich wurde, so etwa durch Schmidt (2018), Fähnrich \& Thein (2018), und Stoll (2018).

Die vorliegende Arbeit fragt danach, wie sich der Flipped Classroom auf das Lernen im schulischen Physikunterricht auswirkt. Um hierüber Aussagen zu treffen, wird diese Methode mit dem traditionellen Unterricht verglichen. Damit ist die Studie eine Methodenvergleichsstudie. Die Bezeichnung »Methode « wird in dieser Arbeit vereinfachend in der Bedeutung eines Unterrichtsformats verwendet. Demnach ist Flipped Classroom eine »Methode «, die der »Methode« des traditionellen Unterrichts gegenübergestellt wird.

Der Einsatz einer Unterrichtsmethode wird durch ihren Kontext begründet. Es gibt nicht die Unterrichtsmethode, die in allen Lerngruppen und für alle Lernziele die besten Ergebnisse zu Tage fördert, sondern nur eine gute oder weniger gute Passung von Methode, Lernzielen, Lernvoraussetzungen und Lerninhalten. Aus diesem Grund ist es ausdrücklich nicht Ziel der Arbeit, einen Methodenwettstreit zu führen, der über die allgemeingültige Überlegenheit der einen über die andere Methode entscheiden soll. In der Analogie von Souvignier \& Gold (2006)

1 In dieser Arbeit wird aus Gründen der besseren Lesbarkeit das generische Maskulinum verwendet, welches das männliche und das weibliche Geschlecht gleichermaßen einschließt. 
entspricht das der Suche nach dem besten Sportler - eine Suche, die aufgrund der Unbestimmtheit der Sportart und der Sportlerklasse zu keinem eindeutigen Ergebnis kommen wird. So, wie die Sportart (etwa Ringen) und die Sportlerklasse (etwa Mittelgewicht) für einen Vergleich bestimmt sein müssen, müssen für eine empirische Methodenvergleichsstudie Lernziel, -voraussetzungen und -inhalt benannt werden, auf die der Vergleich angewendet werden soll.

In der Unterrichtsreihe, in der der Einsatz des Flipped Classroom getestet wurde, sollte vor allem das deklarative Wissen gefördert werden. Damit lag das Lernziel beim physikalischen Fachwissen, das zum Vergleichskriterium wird. Neben dem kognitiven Lernergebnis wurden aber auch affektive Lernergebnisse untersucht, also die Auswirkungen auf die Motivation, das Interesse und das Selbstkonzept der Schüler. Die Lernvoraussetzungen, die für die Methode berücksichtigt werden mussten, waren zum großen Teil durch die Jahrgangsstufe bestimmt. Aus inhaltlichen und organisatorischen Gründen, die in Kap. 4 erläutert werden, fiel die Wahl auf die 11. Jahrgangsstufe, die in Bayern, wo die Studie durchgeführt, zur differenzierten Oberstufe gehört.

Der Physikunterricht der 11. Jahrgangsstufe ist noch aus einem weiteren Grund für die Studie ausgewählt worden. Seit der Einführung des achtjährigen Gymnasiums ist es die Erfahrung vieler Physiklehrer, dass die Physikleistungen der Schüler beim Übergang von der 10. in die 11. Jahrgangsstufe schlechter werden. Dieser Eindruck artikuliert sich bei gymnasiumsübergreifenden Fachsitzungen und regionalen Fachbetreuertagungen. Ein Notenvergleich in der Stichprobe, die für die vorliegende Studie herangezogen wurde, bestätigt diese Beobachtung quantitativ. Die Physiknoten der Schüler im Halbjahreszeugnis der 11. Jahrgangsstufe waren signifikant schlechter als noch ein halbes Jahr zuvor im Jahresendzeugnis der 10. Klasse. ${ }^{2}$ Das ist umso überraschender, als dass die Physikkurse der Oberstufe eine Positivauslese an Schülern bieten, die eine freie Wahlentscheidung für das Fach Physik getroffen haben. Zudem gibt es im achtjährigen bayerischen Gymnasium keine Leistungskurse mehr. Das bedeutet, dass leistungsstarke und motivierte Schüler, die früher Physikleistungskurse gewählt hätten, sich nun in dreistündigen Einheitsphysikkursen der Oberstufen befinden, welche das Anforderungsniveau der früheren Grundkurse besitzen. Die Gründe für den Leistungsabfall im Physikunterricht der Oberstufe liegen mutmaßlich in einer erhöhten Lehrplan- und Stoffdichte, einem zunehmend instruktiven und lehrerzentrierten Unterricht, kürzeren Phasen der Anwendung und Vertiefung und einer unterentwickelten Hausaufgabendisziplin. Die genauen Gründe zu erheben liegt nicht im Rahmen der vorliegenden Studie, doch scheinen gerade im Physikunterricht der Oberstufe Voraussetzungen vorzuliegen, die für den Versuch sprechen, Flipped Classroom einzusetzen: Auslagerung von Instruktion in Lernvideos, Freisetzen von Unterrichtszeit für das aktive Anwenden des Wissens, wechselseitige Hilfestellung durch Mitschüler und individuelle Begleitung durch den Lehrer.

2 Für den Vergleich wurde die Punkteskala 0-15 der Oberstufe in die Notenskala 1-6 der Mittelstufe umgerechnet. Danach sanken die Noten von $M_{10}$. fgst $=2.36(S D=.889)$ auf $M_{11}$. fgst $=2.79(S D=1.112), F(1,140)=9.051$, $p<.001, \eta_{p}{ }^{2}=.218$. Der Effekt ist nach Cohen (1988) sehr groß. 
Der »traditionelle « Physikunterricht der Oberstufe, der in Kap. 5.2 näher beschrieben wird, ist nach Erfahrung von Physiklehrern fachwissensorientierter und stärker instruktionsgestützt als in der Mittelstufe. Damit ist er leichter einheitlich zu klassifizieren als in der Mittelstufe, wodurch die Vergleichskategorie »traditionell« gegenüber »Flipped Classroom« gerechtfertigt werden kann. Der Begriff »Treatment « wird in dieser Arbeit durchgängig als Synonym für die Intervention mit der Methode Flipped Classroom verwendet. »Kontrollgruppe« bezeichnet dem gegenüber die Schüler, die traditionell unterrichtet werden.

Flipped Classroom ist keine physikspezifische Methode. Wo liegt das Interesse einer physikdidaktischen Auseinandersetzung? Es ist ein Anliegen der Physikdidaktik, Physikunterricht weiterzuentwickeln. Dabei werden auch didaktische Entwicklungen aufgegriffen, die außerhalb der Physik entstehen, um sie für die Physikausbildung nutzbar zu machen, wenn es konvergente Ziele gibt. Der innovative Charakter des Flipped Classroom liegt darin, niederschwellige motivationale Anreize für die häusliche Unterrichtsvorbereitung zu setzen und die klassisch im Unterricht verortete Instruktion zeitlich und räumlich aus dem Unterricht auszulagern. So kommt es zu einer Veränderung der unterrichtlichen Aktivitäten von Lehrern und Schülern. Die Frage, ob sich dadurch auch im Physikunterricht positive Effekte für den kognitiven und affektiven Lerngewinn ergeben, entspricht einem originären Interesse der Physikdidaktik.

Die Adaption des Flipped Classroom für den Physikunterricht erfordert vor allem die Berücksichtigung realer Experimente, um wissenschaftsdidaktisch authentisch zu sein. Hier liegt die praktische Herausforderung für den Flipped Classroom. Der Anspruch, reale Experimente als Ausgang, Mittel und Kriterium naturwissenschaftlicher Erkenntnisgewinnung zu betrachten, führt zwingend dazu, ihnen auch im Flipped Classroom Raum zu geben - in den Lernvideos oder in der Unterrichtsaktivität der Schüler. Diesen Anspruch löst die vorliegende Studie ein, indem videografierte Realexperimente in alle Lernvideos aufgenommen wurden. Durch die Ausrichtung an den spezifischen Fachinhalten und realen Experimenten wird Flipped Classroom zu einer physikadäquaten Unterrichtsmethode.

Der Einsatz von Flipped Classroom im deutschen Schulsystem war bisher noch nicht Gegenstand fachdidaktischer Forschung, trotz zunehmender Rezeption vor allem im Mathematikunterricht. Für den Physikunterricht im Speziellen gibt es kaum Hinweise darauf, dass diese Methode an deutschen Schulen auch nur phasenweise eingesetzt wird. Die physikdidaktische Auseinandersetzung mit dem Einsatz des Flipped Classroom im Physikunterricht ist daher bislang noch nicht unternommen worden. Die vorliegende Arbeit soll hierfür einen substanziellen Ausgangspunkt bieten.

Die Arbeit gliedert sich in die folgenden Kapitel: In Kap. 2 wird der Zusammenhang von Lernen, Leistung und affektiven Lernmerkmalen dargestellt, weil er die theoretische Grundlage für den Einsatz des Flipped Classroom bildet. Dabei wird die Wahl der Variablen begründet, die im Rahmen der Studie erhoben und analysiert wurden. Anschließend wird der Flipped Classroom als Unterrichtsmethode und Forschungsgegenstand beleuchtet. In Kap. 3 werden die Forschungsfragen und die sich hieraus ergebenden Hypothesen erläutert und begründet. 
Kap. 4 stellt die unterrichts- und interventionsrelevanten Entscheidungen und Lernmaterialien vor, auf die sich die Studie stützt. Eine umfassende Beschreibung des Untersuchungsdesigns, der Vorgaben für den traditionellen Unterricht wie für das Treatment, der Messinstrumente und der Auswertungsmethoden erfolgt anschließend in Kap. 5. In Kap. 6 werden die Ergebnisse der quantitativen Erhebung vorgestellt und nacheinander, in der Reihenfolge der Forschungsfragen, diskutiert. Abschließend werden in Kap. 7 die Ergebnisse gebündelt zusammengefasst, die Studie kritisch reflektiert und weitergehende Perspektiven des Flipped Classroom und der Folgeforschung beschrieben. 


\section{Lernen und Flipped Classroom - eine theore- tische Fundierung}

In diesem Kapitel wird die Unterrichtsmethode Flipped Classroom lernpsychologisch und fachdidaktisch verortet. Dazu erfolgt zunächst ein Überblick über die Bedeutung schüleraktivierenden Lernens, zu dessen Formen Flipped Classroom gezählt werden kann.

\subsection{Schüleraktivierung und erfolgreiches Lernen}

Wann gelingt guter Unterricht? Nach Meyer (2016, S. 13) ist Unterricht gut, wenn dort »eine sinnstiftende Orientierung und ein Beitrag zur nachhaltigen Kompetenzentwicklung aller Schülerinnen und Schüler geleistet wird«. Um die Bedingungen für das Gelingen guten Unterrichts zu beschreiben, unterscheiden Kunter \& Voss (2011) in Anlehnung an Oser \& Baeriswyl (2001) zwischen Sichtstrukturen, welche den Unterricht äußerlich kennzeichnen, und Tiefenstrukturen, welche die Qualität von Unterrichtsprozessen darstellen. Zu den Sichtstrukturen zählen formale und damit leicht identifizierbare Organisationsmerkmale wie Lehrmethoden, Sozialformen oder Lernmedien. Die Tiefenstrukturen hingegen sind zwar in Sichtstrukturen realisiert, drücken aber die vertikale Qualität der damit verbundenen Lerngelegenheiten aus. Sie umfassen drei Dimensionen (Kunter \& Voss 2011):

(a) die Effizienz der Klassenführung,

(b) das Potenzial zur kognitiven Aktivierung und

(c) die konstruktive Unterstützung der Lernenden.

Diese Tiefenstrukturen zu schaffen ist Aufgabe und Herausforderung der Lehrperson. Es erfordert ihre professionelle Gestaltungskompetenz, Bedingungen für Lernprozesse zu ermöglichen, die guten Unterricht ausmachen. Von den Tiefenstrukturen wird nun die kognitive Aktivierung herausgegriffen werden, weil sie für die Einbettung des Flipped Classroom die größte Relevanz besitzt.

Kognitive Aktivierung oder Schüleraktivierung wird auch in der pädagogischen Psychologie als zentrale Kategorie guten Unterrichts angesehen (Gudjons 2014; Hasselhorn \& Gold 2017; Klippert 2008; Meyer 2016). Damit bezeichnet man jede schulische Lernform, bei der der instruktive Frontalunterricht zurücktritt und die Eigentätigkeit der Schüler in den Vordergrund rückt (Andrews et al. 2011). Der Begriff der Aktivität bezieht sich dabei nicht auf jede beliebige Form der Selbsttätigkeit, wie etwa der verhaltensbezogenen, sondern explizit auf die mentale Selbsttätigkeit (Hasselhorn \& Gold 2017). Die Lehrperson kann Prozesse der kognitiven Aktivierung anstoßen und fördern, indem sie die Schüler mit herausfordernden Aufgaben konfrontiert, kognitive Konflikte provoziert, die Schüler anregt, ihre Gedanken, Konzepte, Ideen und Lösungswege darzulegen und zu erläutern, anregende Fragen stellt und 
generell eine diskursive Unterrichtskultur pflegt (Lipowsky 2009). Aus lernpsychologischer Sicht werden durch kognitive Aktivierung vorhandene Wissensstrukturen der Schüler verändert, erweitert, vernetzt, umstrukturiert oder neu gebildet (Kunter \& Voss 2011). Daraus resultiert ein höherer Lernerfolg. Hake (1998) untersuchte in einer Studie den Effekt von aktivierenden gegenüber traditionellen Lehrmethoden in 62 Einführungskursen der Physik mit $\mathrm{N}=6542$ Teilnehmern und stellt einen mehr als doppelt so hohen Lernzuwachs fest: ${ }^{3} \mathrm{~g}_{a k t}=.48$, $S D=.14 ; g_{\text {trad }}=.23, S D=.04$. Auch auf Basis der TIMSS-Videostudie konnte ein positiver $\mathrm{Zu}$ sammenhang zwischen kognitiver Aktivierung der Lernenden und dem Lernzuwachs gezeigt werden (Klieme et al. 2001).

Dem gegenüber gibt es jedoch Ergebnisse vereinzelter Studien, in den keine direkten Beziehungen zwischen aktivierenden Methoden und dem Lernergebnis gefunden werden konnten (Andrews et al. 2011). Dadurch ist ersichtlich, dass Lernprozesse multidimensional und komplex sind. Schon einzelne Störfaktoren können zu starker Beeinträchtigung führen. Andrews et al. (2011) führen die Abweichung in ihrer Studie auf die unterschiedlichen Voraussetzungen der beteiligten Lehrpersonen zurück.

Ob Schüler die Lernformen annehmen, die ihnen im Unterricht angebotenen werden und die zu kognitiver Aktivierung und Tiefenlernen führen, hängt wesentlich von ihrer Motivation ab. Sie ist sowohl Voraussetzung als auch Ergebnis schüleraktivierender Lernformen. Auf ihre Rolle wird nun detaillierter eingegangen.

\subsection{Motivation, Interesse, Selbstkonzept und Lernleistung}

Allgemein ist Motivation definiert als »die Bereitschaft einer Person, sich intensiv und anhaltend mit einem Gegenstand zu beschäftigen «(Hasselhorn \& Gold 2017, S. 101). Im Kontext des Lernens ist Motivation die Bereitschaft, sich Lernanforderungen zu stellen, sich ihnen fokussiert zu widmen und mit Ausdauer und Anstrengung dabei zu bleiben - kurz: kognitiv aktiv zu sein. Kognitive Aktivierung kann nur gelingen, wenn die motivationalen Voraussetzungen gegeben sind, sonst werden die Lernergebnisse nicht befriedigend sein. Deshalb ist Motivation ein zentraler affektiver Bedingungsfaktor schulischer Lernergebnisse (Krapp 2003).

\subsubsection{Motivation in der Selbstbestimmungstheorie}

Eine erste, eher temporale Differenzierung der Motivation besteht in der Einteilung in aktuelle und habituelle Motivation. Während die aktuelle Motivation die konkrete, situationsbezogene und zeitlich begrenzte Bereitschaft einer Person kennzeichnet, sich auf einen Lerngegenstand

3 Gemessen wurde der mittlere normierte Gain Score g, der das Verhältnis des tatsächlichen zum theoretisch möglichen durchschnittlichen Punktezugewinnes im standardisierten pre/post-Fachwissenstest FCI beschreibt.

4 Im Kontext schulischen Lernens verwendet diese Arbeit den zunächst unbestimmten Begriff ,Motivation' stets im Sinne der ,Lernmotivation'. 
einzulassen, versteht man unter habitueller Motivation das wiederholte beziehungsweise gewohnheitsmäßige Auftreten aktueller Motivation (Schiefele 2009). Die habituelle Motivation an sich ist kein dispositionales Persönlichkeitsmerkmal, hat jedoch Rückbindungen zu anderen dispositionalen Merkmalen, etwa den Interessen, Wert- und Zielüberzeugungen (Pekrun 1988). In der pädagogischen Forschung steht eher die habituelle Motivation im Vordergrund (Schiefele 2008). Sie ist stabil, aber formbar, und bietet für den nachhaltigen Lernerfolg die ergiebigere Grundlage.

Eine weitere, eher lokale Differenzierung der Motivation geschieht durch das Begriffspaar intrinsisch und extrinsisch. Liegt das Ziel einer Lernhandlung in der Durchführung selbst, etwa aus reinem Interesse am Lerngegenstand oder aus Freude am Lernen, so spricht man von intrinsischer Motivation. Extrinsisch hingegen ist eine Motivation, wenn die Durchführung der Lernhandlung auf ihre Folgen gerichtet ist, etwa auf gute Noten, Kompetenzerweiterung, Lob durch den Lehrer, Vermeidung unangenehmer Folgen, soziale Anerkennung oder Karriereabsichten (Pekrun \& Schiefele 1996). Intrinsische Motivation ist für den Lernprozess von hoher Bedeutung, denn Schüler, die in einer Lernsituation intrinsisch motiviert sind, sind in hohem Maße kognitiv aktiviert. Sie lernen hauptsächlich aus Neugier und Interesse am Gegenstand, ohne dass äußere Anreize notwendig sind. Intrinsische und extrinsische Motivation schließen sich nicht gegenseitig aus. Sie können koexistieren, sind aber voneinander abgrenzbar (Amabile et al. 1994; Buff 2001).

Die Selbstbestimmungstheorie von Deci \& Ryan (2002) geht davon aus, dass der Ursprung intrinsischer Motivation dort liegt, wo das Grundbedürfnis des Menschen nach Kompetenz, Selbstbestimmung und sozialer Eingebundenheit gedeckt wird. Die Erfüllung dieser Grundbedürfnisse sorgt für ein positives emotionales Erleben, wodurch Voraussetzungen geschaffen werden, dass intrinsische Motivation wachsen kann (Klieme et al. 2008). Schiefele (2009) führt aus, dass im Gegenzug dort, wo das Erleben von Kompetenz und Selbstbestimmung eingeschränkt ist, auch die intrinsische Motivation nachlässt.

Die intrinsische Motivation ist im schulischen Kontext nicht die dominante Lernmotivation (Kunter 2005; Rakoczy et al. 2008). Die schulischen Lernanforderungen sind von den Schülern in der Regel nicht selbst gesucht, sondern werden von außen durch ein Curriculum an sie herangetragen. Die oben erwähnte Selbstbestimmungstheorie von Deci \& Ryan (2002) differenziert die dichotome extrinsische und intrinsische Motivation weiter und beschreibt graduelle Übergange. So gibt es Formen extrinsischer Motivation, in denen Normen, Einstellungen und Handlungsziele so stark internalisiert sind, dass sie in ihrer Erlebensqualität vergleichbar der selbstbestimmten intrinsischen Motivation sind (Deci \& Ryan 2002; Rakoczy et al. 2008). Je stärker die drei prämotivationalen Grundbedürfnisse nach Kompetenz, Selbstbestimmung und befriedigenden Sozialbeziehungen verwirklicht werden können, desto mehr ist eine Person bereit, eine Handlung auszuführen, zu der sie zunächst nicht intrinsisch motiviert ist. Nach und nach kann dabei die Ebene der sogenannten identifizierten Motivation erreicht werden, wenn das Maß an erlebter Kompetenz, Selbstbestimmung und sozialer Eingebundenheit zunimmt. Hier ist ein wichtiger Hebelpunkt für pädagogisch-didaktisches Handeln in der Schule zu sehen. Wo es unterrichtlich gelingt, Lernprozesse anzustoßen, in denen Schüler 
Kompetenz, Selbstbestimmung und soziale Eingebundenheit erfahren, sind motivationale Voraussetzung für kognitive, aber auch metakognitive Aktivierung geschaffen. Empirische Belege, dass intrinische Motivation wächst, wenn die Grundforderungen der Selbstbestimmungstheorie erfüllt sind, sind zahlreich (Berger \& Hänze 2004; Daniels 2008; Klieme et al. 2001; Krapp \& Prenzel 2011; Lewalter \& Schreyer 2000; Zahay et al. 2017; Zimmerman 2000).

\subsubsection{Interesse: individuell, situational, Sach- und Fachinteresse}

Die Selbstbestimmungstheorie gibt Auskunft über die formalen Bedingungen der Motivation und Schüleraktivierung. Die inhaltliche Bedingung und Ursache der Motivation ist hingegen das Interesse eines Schülers (Daniels 2008; Schiefele 2009). Interesse kennzeichnet allgemein die Beziehung einer Person zu einem Gegenstand (Krapp 1992) und ist damit ebenfalls eine affektive Größe. In ihrer Orientierung unterscheiden sich Interesse und intrinsische Motivation deshalb nicht, denn jedes intrinsisch motivierte Verhalten basiert auf Interesse. Wer sich aus Interesse eine Wissenschaftssendung ansieht, handelt intrinsisch motiviert. Wer sie ansieht, weil es Hausaufgabe war, handelt extrinsisch motiviert. Für Tobias (1994) sind Interesse und intrinsische Motivation praktisch Synonyme. Inhaltlich lassen sie sich fast identisch operationalisieren.

Wie auch bei der Motivation unterscheidet man zwei Arten von Interesse. Beide lösen intrinsische Motivation aus. Das individuelle Interesse ist eine zeitlich relativ stabile, endogene Gegenstandspräferenz, wohingegen das situationale Interesse durch exogene Reize ausgelöst werden kann. Sie sind die äußere Erscheinung des Lerngegenstands, die sich etwa in einer geschickten didaktischen Aufbereitung zeigt (Krapp 1992). Das situationale Interesse ist demnach eher die Wirkung der »Interessantheit « von Lernmaterialien, während sich das individuelle Interesse als Merkmalsausprägung verstehen lässt, die eine Person mitbringt. Nach Hidi \& Renninger (2006) gibt es jedoch auch zwischen diesen beiden Interessensausprägungen einen mehrstufigen Übergang, den sie als Vier-Phasen-Modell der Interessensentwicklung bezeichnen. Das Modell hilft verstehen, welche Möglichkeiten die Unterrichtsgestaltung bei der Entwicklung des Interesses und der Motivation besitzt.

Die erste Phase der Interessensentwicklung ist das ausgelöste situationale Interesse, das etwa durch kognitive Konflikte, attraktive Lernumgebungen oder neuartige Unterrichtsformen und -medien provoziert wird (Hidi \& Renninger 2006). Mitchell (1993) spricht von der catchPhase. Hier wird das Interesse geweckt. Die zweite Phase ist das aufrechterhaltene situationale Interesse, das durch zunehmende Eigentätigkeit und Aktivität der Schüler stabilisiert wird. Diese Phase heißt auch hold-Phase (Mitchell 1993). Aufgaben, deren Relevanz sich den Schülern erschließt, herausfordernde kognitive Aktivitäten sowie projektbasiertes und kooperatives Lernen unterstützen demnach die Entwicklung des Interesses (Hidi \& Renninger 2006; Hoffmann 2002; Mitchell 1993; Schraw \& Dennison 1994). Hier zeigt sich deutlich, dass Interesse, Motivation und Schüleraktivität gekoppelte Konzepte sind, die in gegenseitiger Abhängigkeit und Beeinflussung stehen. Diese ersten beiden Interessensphasen liegen in besonderer 
Verantwortung der Lehrperson, da hier die äußeren, exogenen Anreize des Unterrichts die dispositionalen, endogenen Lernmerkmalen überwiegen. Die dritte Phase ist die des aufkommenden individuellen Interesses. Hier beginnt eine Eigendynamik in der Interessensentwicklung, bei der durch Wissenszuwachs sowie gefühls- und wertbezogene Valenzüberzeugungen im Schüler ein endogenes Interesse keimen kann. In dieser Phase ist noch eine Unterstützung von außen durch das soziale Umfeld nötig, um die positive emotionale Konnotierung zu festigen. Das weit entwickelte individuelle Interesse der vierten Phase ist ein ausgesprochenes Eigeninteresse, das den Schüler mit großer Neugier Fragen stellen lässt, ihm Ausdauer und Energie verleiht und ihn Widerstände zu überwinden hilft (Hidi \& Renninger 2006; Izard et al. 2000). Die unmittelbare Verbindung des weit entwickelten individuellen Interesses und der intrinsischen Motivation liegt auf der Hand.

Das Vier-Phasen-Modell bildet keinen Automatismus ab, sondern ist ein theoretisches Modell, in dem eine potentielle Interessensentwicklung beschrieben wird. Für das unterrichtliche Wirken in der Schule ist es von Bedeutung, weil deutlich wird, dass äußere Anreize der Lernumgebung die individuelle Interessensbeziehung der Schüler zum Unterrichtsinhalt performativ beeinflussen können. Lernangebote, die situationales Interesse wecken, können unter geeigneten Umständen die Basis für aufkommendes und anhaltendes Interesse bilden, welches die motivationale Bedingung schulischen Lernens ist (Krapp \& Prenzel 2011). Krapp (1998) macht deutlich, dass Lehrkräfte an allgemeinbildenden Schulen nur selten auf ein bereits bestehendes und entwickeltes Sachinteresse ihrer Schüler zurückgreifen können. Vielmehr seien sie darauf angewiesen, situationales Interesse erst herzustellen und über gewisse Zeit aufrechtzuerhalten. Hierin verbirgt sich eine eigene Zieldimension von Unterricht. Beispielhaft sei hierfür der Bayerische LehrplanPLUS angeführt, der fordert, dass der flexible Einsatz verschiedener, auf die Lernsituation abgestimmter Unterrichtsmethoden Lernmotivation, Interesse und Konzentration der Schüler fördern solle (BStMUK, Bayerisches Staatsministerium für Unterricht und Kultus 2015, Kap. 3.3, Abs. 3).

Während das Interesse bisher als allgemeines psychologisches Konstrukt für schulische Lernprozesse betrachtet wurde, soll nun das physikbezogene Interesse in den Blick genommen werden. In Anlehnung an frühere Studien trafen Hoffmann et al. (1998) für die IPN-Interessenstudie die Unterscheidung zwischen Interesse an Physik im Allgemeinen und Interesse am Unterrichtsfach Physik, also von Sachinteresse und Fachinteresse. Dabei bezieht sich das Sachinteresse ausschließlich auf physikalische Themen und steht damit dem individuellen Interesse nahe. Das Fachinteresse hingegen ist eine Kombination aus drei Faktoren,

(a) dem individuellen Sachinteresse,

(b) dem unmittelbaren, situationalen Interesse, ausgelöst durch die didaktisch unterstützte Interessantheit des Lerngegenstandes und

(c) dem sozialen Lernklima in der Physikklasse, womit sowohl die Schüler-Schüler- als auch die Lehrer-Schüler-Beziehung gemeint ist (Hoffmann 2002; Hoffmann et al. 1998).

Die Gewichtung der einzelnen Faktoren variiert individuell zwischen Schülern, so dass ein hohes Fachinteresse mit einem niedrigen Sachinteresse einhergehen kann und umgekehrt 
(Hoffmann 2002). Aufgrund der verschiedenen Einflussgrößen kann das Fachinteresse eine zeitlich größere Schwankung besitzen als das Sachinteresse. Zwar misst die IPN-Studie beispielsweise für die 8. Jahrgangsstufe eine relativ hohe Korrelation zwischen Sach- und Fachinteresse mit $r=.57$ (Hoffmann et al. 1998), aber Häussler \& Hoffmann (2000) zeigen in einer regressionsanalytischen Untersuchung des Fachinteresses auch, dass der stärkste Prädiktor für das Fachinteresse nicht das Sachinteresse, sondern das physikbezogene Selbstkonzept ist $(\beta=.43)$, welches eng an die Notenleistung gekoppelt ist, gefolgt von der Stimulation des Interesses durch die Lehrkraft $(\beta=.17)$. Wenn die erwartete Notenleistung oder Fremdbestätigung ausbleibt, es Verstimmungen in der Lehrer-Schüler-Beziehung gibt, die Lehrkraft eine Unterrichtsreihe ungeschickt aufbereitet oder andere ungünstige Lernbedingungen herrschen - räumlich oder zeitlich -, kann das zu einem mittelbaren Absinken von Fachinteresse und Motivation führen, ohne dass das Sachinteresse involviert wäre. Tatsächlich besitzt das Sachinteresse mit $\beta=.08$ eine überraschend geringe Vorhersagekraft für das Interesse am Unterrichtsfach Physik. Hieraus ziehen Häussler \& Hoffmann (2000) die Schlussfolgerung, dass der Großteil des Physikunterrichts inhaltlich an den physikalischen Sachinteressen der Schüler vorbeigeht.

In Längsschnittstudien wird immer wieder gezeigt, dass das Interesse an Schulfächern im Laufe der Schulzeit kontinuierlich sinkt, wovon besonders stark die naturwissenschaftlichen Fächer Physik und Chemie sowie die Mathematik in der Unter- und Mittelstufe betroffen sind (Daniels 2008; Hannover \& Kessels 2001; Hidi 2000; Hoffmann et al. 1998; Kleickmann 2011; Krapp 2002; Wild \& Hofer 200o). Die Abnahme des Fachinteresses ist dabei bei den Mädchen stärker als bei den Jungen (Hoffmann 2002; Krapp 2006). Die vorliegende Studie bezieht sich auf Schüler der 11. Jahrgangsstufe. Diese haben bereits die Fächerwahl für die Oberstufe getroffen. Die Lernmotivation und die Interessenswerte von Schülern - Jungen wie Mädchen -, die freiwillig Physik in der Oberstufe belegen, sollte über dem Durchschnitt der Schüler in der Mittelstufe liegen. Es wird zu untersuchen sein, ob sich das Interesse von Jungen und Mädchen während der Studie unterschiedlich entwickelt.

Neben dem makroskopischen Rückgang an Fachinteresse über Schuljahre hinweg scheint es auch mesoskopische Dynamiken auf kleinerer Zeitskala innerhalb eines Schuljahres zu geben. Die BIJU-Längsschnittstudie mit 3787 Schülern zeigte bereits zwischen drei Messzeitpunkten innerhalb der 7. Jahrgangsstufe einen signifikanten Fachinteressensrückgang über alle Fächer hinweg. Das unterrichtsbezogene Interesse an physikalischen Themen oder Kontexten (das sogenannte topologische Interesse) fiel innerhalb weniger Monate ebenfalls signifikant ab (Daniels 2008). Auch schon in mehrwöchigen Unterrichtsverläufen fallen signifikante Abnahmen von Interessenswerten auf (Weber 2003; Winkelmann 2013). Erklärungen hierfür stehen aus, müssen aber entsprechend auf mesoskopischer Ebene systemimmanent zu suchen sein.

Zusammenfassend ist festzuhalten, dass das Sachinteresse ein der Lernmotivation vorgelagertes inhaltsbezogenes Konzept ist, das von einer eher oberflächlichen, situativen Erscheinung bis zu einem individuellen, dispositionalen Persönlichkeitsmerkmal reichen kann. An 
der Vertiefung des Sachinteresses kann der schulische Unterricht mitwirken. Das Sachinteresse ist deshalb von Bedeutung, weil es - neben dem Erleben von Kompetenz, Selbstbestimmung und sozialer Eingebundenheit - die gegenstandsbezogene intrinsische Lernmotivation der Schüler steuert. Vom inhaltlichen Sachinteresse zu unterscheiden ist das komplexere Fachinteresse. Hier fließen mehrere, zeitlich variable Faktoren ein, die im einzelnen Schüler zu einem Gesamtzustand führen, der sich als Fachinteresse operationalisieren lässt. Ebenso wie das Sachinteresse löst auch das Fachinteresse intrinsische Motivation aus, auch wenn das Fachinteresse kontextuelle Faktoren integriert, die nicht inhaltsbezogen sind.

\subsubsection{Selbstkonzept: schulisch und fachspezifisch}

Das Selbstkonzept ist neben der Motivation und dem Interesse ein weiteres affektives Konstrukt, das für Lernprozesse hohe Relevanz besitzt. Unter dem Begriff Selbstkonzept versteht man die »Wahrnehmung und Einschätzung eigener Fähigkeiten und Eigenschaften « (Hasselhorn \& Gold 2017, S. 112). Die mit dem Selbstkonzept verwandte Selbstwirksamkeit oder Selbstwirksamkeitserwartung ist die subjektiv empfundene Wahrscheinlichkeit, eine neue oder schwierige Situation aufgrund eigener Fähigkeiten meistern zu können (Bandura 1997). Die Selbstwirksamkeitserwartung misst sich also immer an einer konkreten Hürde, die zu nehmen ist, wohingegen das Selbstkonzept eine subjektive mentale Repräsentation der eigenen Fähigkeiten ist. Die Ausbildung eines positiven Selbstkonzepts von Schülern ist eine wichtige Zieldimension unterrichtlichen Handelns, da das psychische Wohlbefinden von Kindern, Jugendlichen und Erwachsenen von einer positiven Selbstbewertung profitiert (Möller \& Trautwein 2009; Stiensmeier-Pelster \& Schöne 2008).

Nach Shavelson et al. (1976) ist das Selbstkonzept hierarchisch und multidimensional geordnet. Wird die Einschätzung des eigenen schulischen Leistungsstands von Schülern in den Blick genommen, spricht man vom akademischen oder schulischen Selbstkonzept oder auch schulischen Fähigkeitsselbstkonzept. Das schulische Selbstkonzept ist untergliedert in Subdimensionen. So lässt sich ein mathematisch-naturwissenschaftliches Selbstkonzept beschreiben, das sich von anderen Subdimensionen, etwa einem sprachlichen Selbstkonzept abgrenzen lässt (vgl. Abb. 1). Diese Differenzierung setzt sich in den einzelnen Fächern fort und resultiert in fachspezifischen Selbstkonzepten.

Wie auch das Interesse ist das Selbstkonzept ein stabiles, aber formbares Personenmerkmal, das umso leichter beeinflussbar ist, je tiefer es sich in der Hierarchie befindet und je spezialisierter es ist. (Demo \& Savin-Williams 1992; Markus \& Kunda 1986; Shavelson et al. 1976). Diese Tatsache lässt nach der Möglichkeit fragen, in welchem Maße die Lehrperson und die Unterrichtsgestaltung Einfluss auf das fachspezifische Selbstkonzept nehmen können.

Es gibt drei Bezugsnormen, an denen sich ein Schüler orientiert und so ein fachspezifisches Selbstkonzept generiert: 


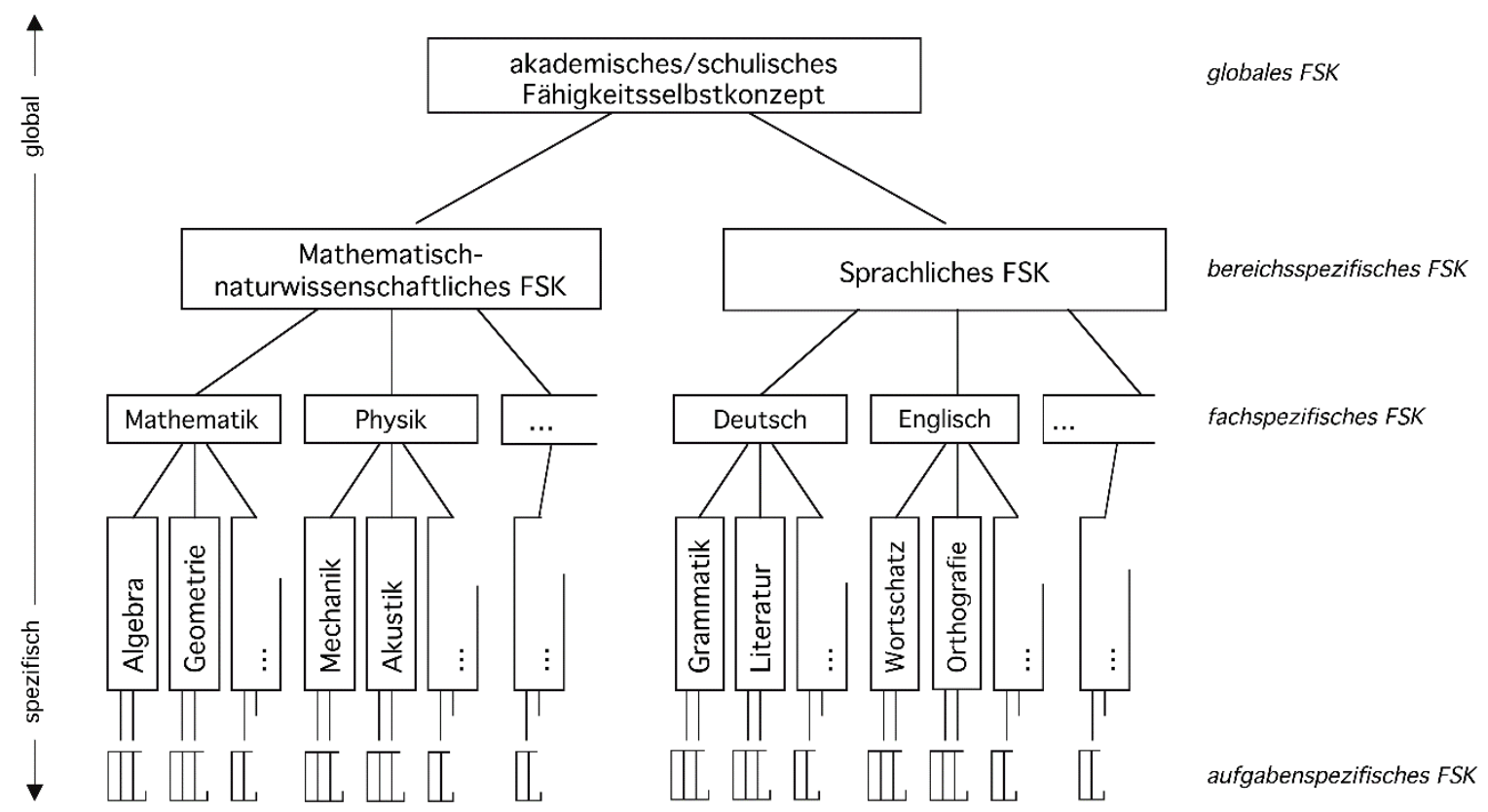

Abb. 1. Hierarchische Struktur des Selbstkonzepts (Stiensmeier-Pelster \& Schöne 2008, S. 63)

(a) Die sachliche oder kriteriale Bezugsnorm für einen Schüler ist die Fremdbewertung der Leistung durch die Lehrperson, die eine gewisse Leistungserwartung besitzt. Der sogenannte Skill-Development-Ansatz sieht formatives oder normatives Feedback durch die Lehrperson als wirkursächlich für die Genese fachspezifischer Selbstkonzepte an. Noten prägen demnach das Selbstkonzept.

(b) Die soziale Bezugsnorm für einen Schüler bilden die Leistungen und Fähigkeiten der Mitschüler. Schüler messen sich demnach an den Leistungen einer Referenzgruppe.

(c) Die individuelle Bezugsnorm für einen Schüler ist die eigene Leistung zu einem früheren Zeitpunkt (temporaler Vergleich) oder in anderen Fächern (dimensionaler Vergleich) (Stiensmeier-Pelster \& Schöne 2008).

Es wird deutlich, dass sich das Selbstkonzept in einem Spiel von extra- und intraindividuellen Bezügen befindet. Es gibt nur begrenzten unterrichtlichen Gestaltungsspielraum für die Unterstützung eines positiven Selbstkonzepts, der sich auf eine konstruktive Art und Weise der Leistungsrückmeldung durch die Lehrperson beschränkt. Vielmehr ist der Hebelpunkt die Lernleistung selbst, die über Motivation und situationales Interesse gefördert werden kann. Vereinfacht gesagt führt eine hohe Motivation und kognitive Aktivierung zu positiven Lernerfahrungen, welche sich günstig auf das spezifische Selbstkonzept auswirken. In einem Prozess der Selbstverstärkung wirkt sich ein erhöhtes Selbstkonzept auf Interesse, Lern- und Leistungsmotivation aus, so dass auch ein Einfluss des Selbstkonzepts auf die Lernleistung nachgewiesen werden kann (Self-Enhancement-Ansatz) (Köller et al. 2006; Marsh \& Craven 2006; Trautwein et al. 2006; Valentine et al. 2004). 
Die bis hierher betrachteten lernpsychologischen Konstrukte Motivation, Interesse und Selbstkonzept hängen eng miteinander zusammen. Nach Rost \& Sparfeldt (2002) gibt es signifikante Korrelationen zwischen Fachinteresse und fachspezifischem Selbstkonzept bei 564 Schülern der 8. bis 10. Jahrgangsstufe in Deutschland, wobei Physik $(r=.70, p<.01)$ und Mathematik $(r=.72, p<.01)$ die höchsten Korrelationswerte besaßen. Es ist nicht verwunderlich, dass sich die Geschlechterdifferenz des Interesses am Schulfach Physik zwischen Jungen und Mädchen auch im Selbstkonzept widerspiegelt. Bei Rost \& Sparfeldt (2002) wirkte das Geschlecht auf das Selbstkonzept Physik mit einer Effektstärke von Cohen's $d=.77$, was einen starken Effekt kennzeichnet. Da jedoch Jungen durchschnittlich nur geringfügig bessere Notenleistungen im Fach Physik erbringen als Mädchen (Häussler \& Hoffmann 1998; Prenzel et al. 2007; Schiepe-Tiska et al. 2016), gibt es neben der Leistung noch andere, geschlechtsbezogene und sozial vermittelte Einflüsse auf das physikspezifische Selbstkonzept. Anders lässt sich nicht erklären, dass Mädchen mit der Note »gut « in Physik im Mittel das gleiche Selbstkonzept haben wie Jungen mit der Note »befriedigend « (Schilling et al. 2006). Ansätze, auf die niedrigen Interessens- und Selbstkonzeptwerte zu reagieren, sind zeitweilige Monoedukation (Hannover \& Kessels 2001; Herwartz-Emden 2007; Häussler \& Hoffmann 1998; Neumann \& Borowski 2010), Anpassung des Unterrichtsmaterials durch stärker lebensweltliche Kontexte (Hirsch 2005; Häussler \& Hoffmann 1995; Schnirch 2006; Walper 2017) oder Reattributionstraining, das Selbstverstärkungsmechanismen negativer Selbstkonzepte aufbricht (Wodzinski 2007).

Bis hierher wurden die erklärenden Konstrukte kognitiver Aktivierung von Schülern beschrieben: Motivation, Interesse und Selbstkonzept. Sie stehen in einem wechselseitigen Abhängigkeitsverhältnis untereinander. Im nächsten Unterkapitel soll der interdependente $\mathrm{Zu}$ sammenhang der schulischen Lernleistung mit diesen Variablen skizziert werden.

\subsubsection{Schulische Lernleistung}

Der schulische Lernerfolg ist eine multidimensionale Größe. Sie ist das Ergebnis des Lernprozesses im ganzheitlichen Bildungs- und Erziehungsauftrag der Schule. Das spezifische Lernergebnis eines Unterrichtsfachs lässt sich innerhalb der Kompetenzbereiche Fachwissen, Erkenntnisgewinnung, Kommunikation und Bewertung messen. Im Rahmen dieser Arbeit steht der Erwerb des Fachwissens im Vordergrund. Alle bisher vorgestellten affektiven Lernmerkmale - Motivation, Interesse und Selbstkonzept - stehen in Zusammenhang zum kognitiven Fachwissenszuwachs, der hier Lernleistung genannt wird.

Über den Zusammenhang von intrinsischer Lernmotivation und Lernleistung gibt die Metaanalyse von Schiefele \& Schreyer (1994) Aufschluss. In 30 Studien zeigte sich im Mittel eine positive, wenn auch nicht sehr hohe Korrelation von $r=.23$ von fachspezifischer Lernmotivation und Fachnoten beziehungsweise standardisierten Leistungstests. Hattie et al. (2015) geben für den - hier undifferenzierten - Einflussfaktor Motivation auf die Lernleistung eine Effektstärke von $d=.48$ an, was einem mittleren Effekt entspricht. Csikszentmihalyi \& Wong 
(2014) stellen ergänzend heraus, dass nur Lernmotivation, die von positiven Emotionen begleitet ist, zu einem höheren Lernergebnis führt. Extrinsisch motiviertes Lernen aus einem Gefühl der Pflichterfüllung heraus ist kein Prädiktor für gute Leistungen.

Die mittlere Korrelation von Interesse und Lernleistung bei 127 untersuchten Studien zwischen 1965 und 1990 lag bei $r=.30, S D=.134$, für das Fach Physik bei $r=.31, S D=.117$ (Krapp et al. 1993). Dieselbe Metastudie zeigte, dass die Interessensausprägung ungefähr $10 \%$ der beobachteten Varianz erklärt, jedoch ohne Einbeziehung von Moderatoreffekten (Fach, Geschlecht, Fähigkeitsniveau der Probanden). Die IPN-Interessenstudie stellt für die 8. Jahrgangsstufe im Fach Physik eine Korrelation von $r=.38$ zwischen Fachinteresse und Leistung fest (Hoffmann et al. 1998).

Die Beziehung zwischen Selbstkonzept und Lernleistung ist empirisch ebenfalls gut belegt (Baumeister et al. 2003; Marsh \& Craven 2006; Wylie 1979). Die Metastudie von Valentine et al. (2004) bezieht sich auf 6o Längsschnittstudien, die allesamt positive Effekte der Selbsteinschätzung auf schulische Leistungen zeigten. Wie oben dargestellt, beschreiben sich ergänzende Modelle das Verhältnis als reziprok, so dass also sowohl Leistungen ursächlich für Selbstkonzepte sind, als auch umgekehrt Selbstkonzepte Lernleistungen beeinflussen (Köller et al. 2006; Möller \& Trautwein 2009).

Die theoretische Fundierung der PISA-Studie 2015, auf deren Skalen sich die vorliegende Studie fast ausschließlich stützt, verknüpft diese Variablen in einen unauflösbaren Zusammenhang:

»Motivation to learn that is based on interest and enjoyment is experienced as self-determinate and intrinsic.
It affects student engagement, learning activities, performance and career choices, and can be shaped by
classroom instructions and parental motivation practices."

(OECD, Organisation for Economic Co-operation \& Development 2016, S. 109)

Nachfolgend soll Abb. 2 den Zusammenhang der wichtigsten lernpsychologischen Größen abschließend veranschaulichen.

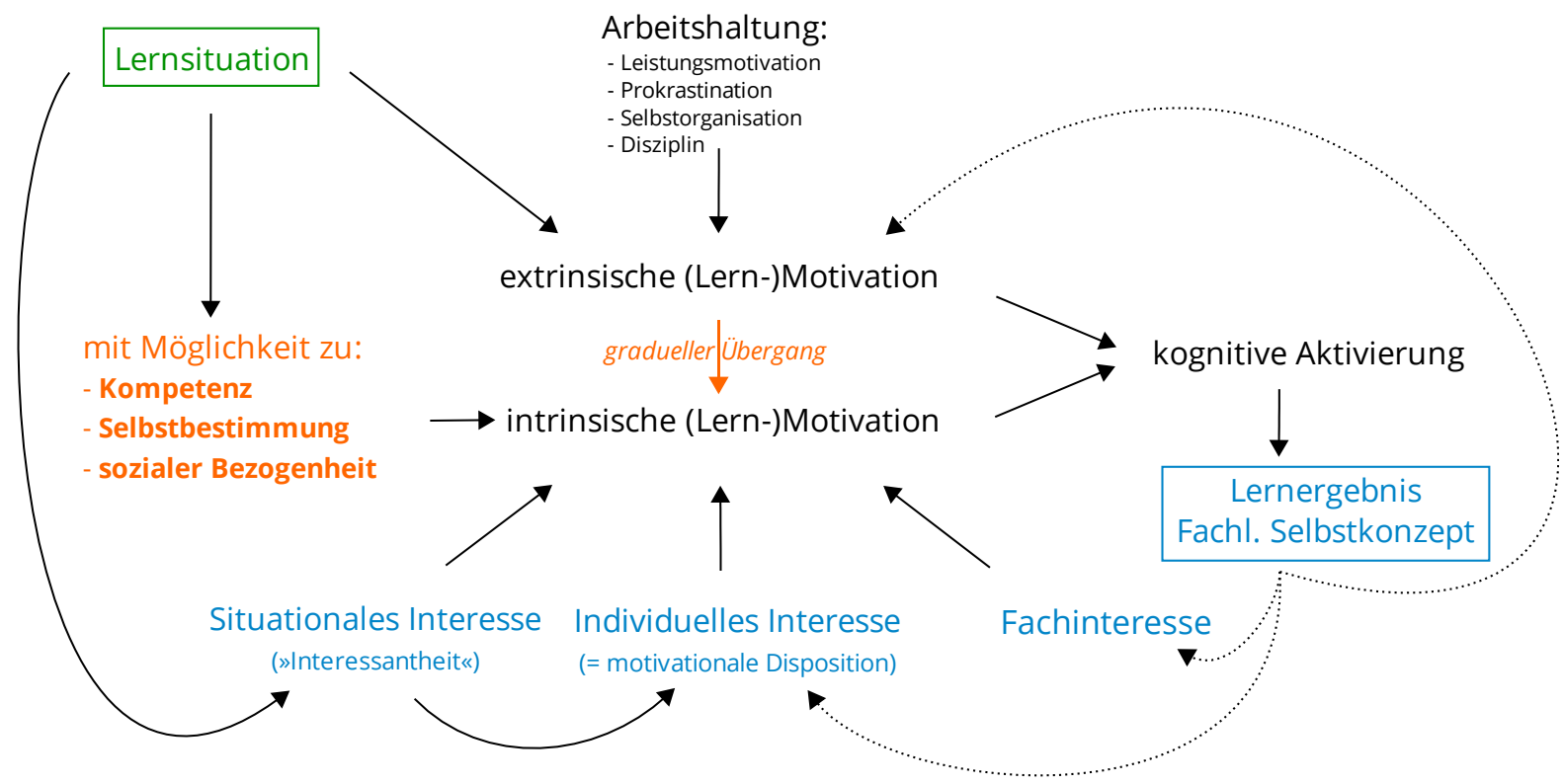

Abb. 2. Zusammenhang lernrelevanter Unterrichtsgrößen 


\subsection{Flipped Classroom als Unterrichtsmethode}

Die ersten beiden Unterkapitel haben auf lernpsychologischer Basis gezeigt, dass erfolgreiches Lernen von kognitiver Aktivierung abhängt. Dabei sind kognitive und affektive Lernprozesse wechselseitig miteinander verbunden. Ausgehend von dieser Erkenntnis wird nun die Unterrichtsmethode Flipped Classroom in Theorie und Praxis beschrieben. Dabei wird gezeigt, dass sie eine in besonderer Weise schüleraktivierende Form des Unterrichtens ist.

\subsubsection{Ursprung und Formen des Flipped Classroom}

Flipped Classroom ist eine methodische Großform, in der die Instruktionsphase aus dem Unterricht in die häusliche Vorbereitung ausgelagert wird, damit im Unterricht mehr Zeit für die selbstständige und vertiefte Auseinandersetzung mit den Lerninhalten zur Verfügung steht. »Flipped «, also umgedreht, wird diese Methode genannt, weil sie die Aktivitäten, die in einem lehrerzentrierten Frontalunterricht in der Schule stattfinden, mit den Aktivitäten, die sich für die Hausaufgabe daraus ableiten, vertauscht. Die Instruktion, die klassischerweise ihren Platz im Unterricht besitzt, erfolgt zuhause, während das Anwenden und Üben, das sonst in den Hausaufgaben geleistet wird, ausschließlich im Unterricht stattfindet. Abeysekera \& Dawson (2015) nennen drei Elemente, die den Kern des Flipped Classroom bilden:

(a) Der Großteil der Inhaltsvermittlung geschieht außerhalb des Unterrichts,

(b) die Unterrichtszeit wird für aktivierende und kooperative Lernformen genutzt und

(c) von den Schülern werden vor- und/oder nachbereitende Tätigkeiten erwartet, um einen größtmöglichen persönlichen Nutzen zu erzielen.

Über welches Medium die Auslagerung der Inhaltsvermittlung geschieht, ist offen. So können etwa Texte oder das Schulbuch als Medium der häuslichen Vorbereitung verwendet werden, mit Hilfe derer die Schüler sich Wissen aneignen sollen, das im Unterricht vertieft wird. Tatsächlich ist der Begriff »Flipped Classroom « in der Praxis meist mit im Internet verfügbaren Lernvideos als Instruktionsmedium verknüpft, weshalb die Methode gern in den Bereich der digitalen Bildung eingeordnet wird. Die Ausbreitung der Methode ist dabei eng an den Ausbau leistungsfähiger Breitbandnetze gebunden. Erst durch eine vorhandene Infrastruktur wird der unkomplizierte Zugang zu den Lernvideos möglich. Die deutsche Bezeichnung »Umgedrehter Unterricht « ist weniger verbreitet.

Die beiden US-amerikanischen Chemielehrer Bergmann \& Sams (2012) gelten als Pioniere des Flipped Classroom, seit sie im Jahr 2006 an der Woodland Park High School in Woodland Park, Colorado, begonnen hatten, die Inhalte ihres Unterrichts als PowerPoint-Folienpräsentationen über eine Screencast-Software aufzuzeichnen und ihren Schülern als Videos online zur Verfügung zu stellen. Etwas früher begann die Entwicklung im Hochschulbereich, wobei hier ganze Vorlesungen aufgezeichnet und online gestellt wurden (Lage et al. 200o). Hier wurde der Begriff »Inverted Classroom « geprägt, der heutzutage synonym verwendet wirdallerdings fast ausschließlich nur noch im deutschen Sprachraum. 
Der Flipped Classroom ist eine Form des blended learning, weil er Elemente des individuellen Online-Lernens mit Präsenzlernen im Klassenzimmer verbindet. Der entscheidende Faktor sind dabei jedoch nicht die Lernvideos, sondern die freigesetzte Unterrichtszeit, die intensiv für kooperative und schüleraktivierende Lernformen genutzt werden kann. Dieser Punkt muss deutlich hervorgehoben werden, weil Flipped Classroom oft einseitig in den Bereich des multimedialen Lernens und digitalen Kompetenzerwerbs eingeordnet wird. Über die online verfügbaren Lernvideos besitzt der Flipped Classroom einen internettechnologischen und multimedialen Aspekt, doch sind die Lernvideos lediglich Vehikel, um zeitliche Ressourcen für das unterrichtliche und lehrerbegleitete Lernen zu schaffen. Nicht das Medium der häuslichen Inhaltsvermittlung steht im Vordergrund, sondern die Formen der unterrichtlichen Inhaltsvertiefung.

Unterschiedliche Formen der Lernvideos und der schulischen Lernmaterialien schaffen eine Vielzahl von Variationen des Flipped Classroom. So können die Lernvideos Aufgaben enthalten, die die Schüler ebenfalls zuhause bearbeiten sollen. Interaktive Lernvideos setzen die Wiedergabe nach einer Haltemarke erst fort, wenn eine Eingabe getätigt wurde. Auch ist es möglich, dass die Schüler die Lernvideos nicht vor- sondern nachbereitend zum Unterricht schauen ( $h a l f f l i p p e d$ ) oder in einer selbstgesteuerten Lernumgebung während des Unterrichts (in-class flipped) (Bergmann 2014; Fryling et al. 2016; Schmidt 2018). Welche Formen der Schüleraktivität im Unterricht gewählt werden, ist von der Lernsituation abhängig. Je nach Erfordernissen können es schriftliche, mündliche oder praktische Lernaufgaben sein. Die Sozialform (Einzel-, Partner-, Gruppen-, Plenararbeit) kann dabei ebenso variieren wie die im Unterricht verwendeten Handlungsmuster und Arbeitstechniken (z.B. Freiarbeit, Lernspiele, themenzentrierte Selbstdarstellungen, Lerntheke, Wochenplan, Arbeiten mit dem Schulbuch).

Verfügen Lehrpersonen über ein ganzes Set von Lernvideos und korrespondierenden Anschlussaufgaben, ist ein Flipped Mastery Learning möglich (Bergmann \& Sams 2014; Said \& Zainal 2017). Hier lernen die Schüler in individueller Geschwindigkeit und rücken zum nächsten Wissensgebiet vor, wenn das vorangehende zufriedenstellend abgeschlossen ist, was durch formative Selbst- und Fremdevaluationen überprüft wird. Das Konzept des Mastery Learning mit seinen kleinen Lerneinheiten und Feedbackschleifen hat eine wesentlich längere Tradition als der Flipped Classroom und geht auf Bloom (1968) zurück. Doch lässt sich das Mastery Learning organisch in den Flipped Classroom einbinden, da die binnendifferenzierte Passung der Instruktion an den Wissensstand des einzelnen Schülers über die Lernvideos und ihre Aufgaben leicht möglich ist. Dieses Konzept ist etwa für den Inklusionsansatz an Schulen vielversprechend (Altemueller \& Lindquist 2017).

Eine wichtige Gelenkstelle zwischen Lernvideo und Unterrichtsaktivität nimmt das Assessment ein. Häufig wird dazu ein Quiz verwendet, das dem Schüler und der Lehrkraft dazu dient, einzuschätzen, wie gut die Vorbereitung erfolgt ist und das Lernvideo verstanden wurde. Es kann als häusliches Online-Fragequiz konzipiert sein, zu dem sich der Schüler im Anschluss an das Video mit einer Kennung einloggen muss (Aşıksoy \& Özdamlı 2016; Lo \& Hew 2017). Nach jeder Frage wird dem Schüler die richtige Antwort zur Kontrolle geboten. Gängiger ist die Praxis, ein Quiz mit konzeptuellen Multiple-Choice-Fragen zu Beginn der 
Unterrichtsstunde durchzuführen. Alle Schüler erhalten dazu ein Abstimmungsinstrument, mit dem sie die ihrer Ansicht nach richtige Antwort auf die präsentierte Frage geben. Ein solches Classroom Response System sind beispielsweise Clicker, Website- oder App-basierte Voting-Möglichkeiten oder im einfachsten Fall verschiedenfarbige Abstimmkarten. Alle funkbasierten Lösungen erleichtern es den Schülern, anonym und unabhängig vom Nachbarn abzustimmen. Das Abstimmungsergebnis wird in Echtzeit der Klasse präsentiert. Ist die Quote der richtigen Antworten hoch genug (zum Beispiel 75\%), wird die Frage aufgelöst und die nächste Frage wird gestellt. Ist die Quote niedriger, wird das als Anlass dafür genommen, dass die Schüler mit ihren Sitznachbarn über die Lösung diskutieren. Anschließend wird erneut abgestimmt und die Frage aufgelöst. Dieses Verfahren wurde von Mazur (1999) als Peer Instruction ursprünglich in der Hochschullehre eingeführt und wurde seitdem in verschiedenen Kontexten rezipiert. Smith et al. (2009) zeigten, dass das Lernen durch Peer Instruction zu konzeptuellen Fragen zu einem höheren Lerngewinn führt als ohne den Austausch mit Mitschülern. Im Flipped Classroom dient die Quizphase auch dazu, offene Fragen zum Lernvideo zu klären, ohne jedoch die Inhalte des Videos noch einmal zusammenfassen. Würden die Inhalte der Videos im Unterricht mündlich wiederholt, hätten die Schüler weniger Veranlassung, die Videos weiterhin zur Vorbereitung anzusehen. Das Quiz bietet die einfachste Möglichkeit, das Wissen der Schüler zu aktivieren, sie in einen Austausch zu bringen, sich gegenseitig zu korrigieren und zu ergänzen. Deshalb wird in den meisten Formen des Flipped Classroom das Quiz als Unterrichtseinstieg eingesetzt, von dem ausgehend schüleraktivierende Lernprozesse unterschiedlicher Form initiiert werden. In den meisten Fällen ist das selbstständiges, kooperatives und differenzierendes Lernen in Paaren oder Gruppen (Bergmann \& Sams 2012; Bishop \& Verleger 2013; Schmidt 2018).

\subsubsection{Gemeinsamer Nenner: Basisform des Flipped Classroom}

Aus den unterschiedlichen Variationen des Flipped Classroom kann eine Basisform herauskristallisiert werden, die eine Art gemeinsame Wurzel darstellt und auch in dieser Studie angewandt wurde. Die Basisform steht in Übereinstimmung mit einer Vielzahl von Praxisbeispielen (Leibniz-Institut für Wissenschaftsmedien 2017; Abeysekera \& Dawson 2015; Ash 2012; Bergmann \& Sams 2012; Brame 2013; Demski 2013; Roehl et al. 2013; Schmidt 2018; Yıldrım \& Kıray 2016) und besteht aus den folgenden drei Elementen (vgl. Abb. 3):

Instruction: Die Instruktionsvideos sind generell eher kurz. Je kürzer, desto höher ist die Wahrscheinlichkeit, dass die Schüler ein Video bis zum Ende sehen. Guo et al. (2014) empfehlen, eine Länge von sechs Minuten Video nicht zu überschreiten. Um die Auseinandersetzung mit den Inhalten zu fördern und eine schriftliche Grundlage für Prüfungsvorbereitungen zu liefern, bieten die Lernvideos am Ende oft eine Zusammenfassung, die die Schüler in ihr Heft übertragen. 


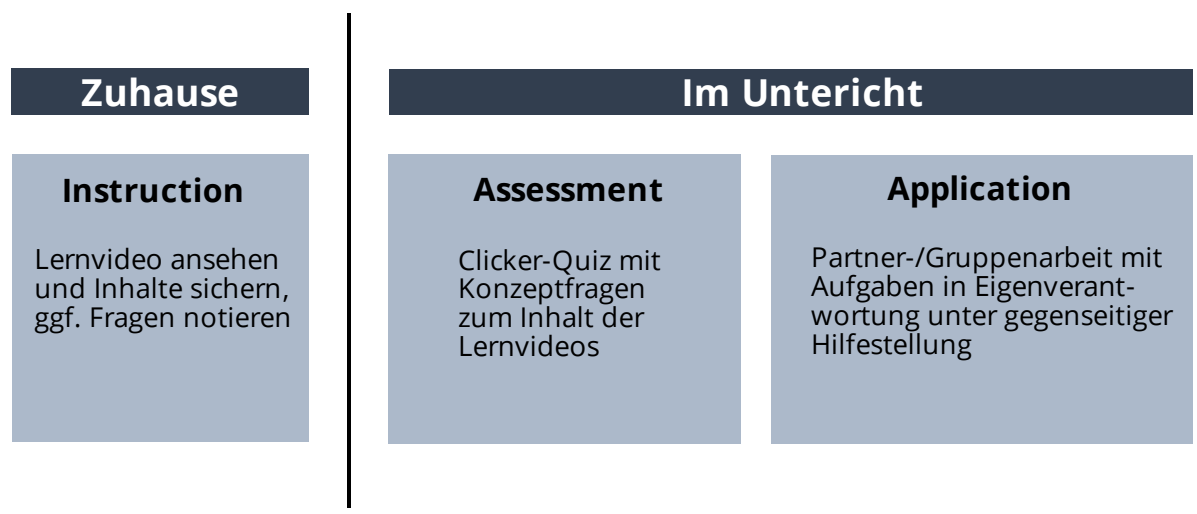

Abb. 3. Basisform des Flipped Classroom

Assessment: Multiple- oder Single-Choice-Konzeptfragen im interaktiven Clicker-Quiz regen die Schüler zu Beginn der Stunde an, die Inhalte des Videos zu rekapitulieren. Die Lehrkraft erhält eine anonyme und direkte Rückmeldung über den Verständnisgrad bei den Schülern. Konzeptfragen eignen sich deshalb besonders, weil sie nicht nur auf Reproduktion der Lerninhalte ausgerichtet sind, sondern auf die dem Video zugrundeliegenden Konzepte und Prinzipien abzielen. Wenn die überwiegende Mehrheit der Schüler die Frage richtig beantwortet hat, wird sie aufgelöst, andernfalls müssen die Schüler sich mit ihren Nachbarn austauschen und im Gespräch zu einer Lösung kommen. Anschließend wird die Frage erneut zur Beantwortung frei gegeben. Ist die Quote richtiger Antworten immer noch zu niedrig, löst die Lehrkraft die Frage mit erklärenden Hinweisen auf. Die Phase des Clicker-Quiz sollte nicht zu viel Zeit in Anspruch nehmen, aber die grundlegenden Inhalte des Lernvideos abdecken.

Application: Nun beginnt die eigentliche Lernaktivität, zu der das Lernvideo und das Clicker-Quiz hinführen. Je nach Einzel- oder Doppelstundenformat steht dabei ein unterschiedliches Maß an Zeit zur Verfügung. In Unterrichtsreihen, die aufgrund ihrer kognitiven Herausforderungen ein hohes Maß an selbsttätigem Üben und Anwenden erforderlich machen, bietet sich das Lösen von Aufgaben und Problemen an. Die Schüler haben dabei ausgiebig Gelegenheit, in kooperativen Arbeitsformen ihr Basiswissen anzuwenden und zu Expertenwissen zu vertiefen. Geschieht das in Partner- oder Gruppenarbeit, können die Schüler sich gegenseitig unterstützen, ihr Wissen in eigene Worte fassen, Teilaspekte erklären, Verständnisschwierigkeiten artikulieren und gegebenenfalls arbeitsteilig agieren. Neben der Förderung der kommunikativen Fähigkeiten, die damit geübt werden, ermöglichen diese Lerngelegenheiten das Erleben von Kompetenz und Bestätigung, aber auch von Angewiesenheit. Die Hemmschwelle, im informellen Rahmen Fragen zu stellen und Unwissen zu offenbaren, ist in der Peer Instruction wesentlich niedriger (Mazur 2014), so dass durch die Schüler-Schüler-Interaktion ein hohes Maß an kognitiver Aktivierung und Lernfortschritt erzielt werden kann. Um dem unterschiedlichen Lerntempo der Arbeitsgruppen gerecht zu werden, bietet es sich an, Musterlösungen und Erwartungshorizonte an zentraler Stelle auszulegen, damit die Schüler ihre Ergebnisse selbstständig überprüfen können. Exemplarische Schlüsselprobleme können auch gemeinsam an der Tafel besprochen werden, wenn sie übergeordnete Bedeutung für den weiteren Lernfortschritt besitzen. 


\subsubsection{Theoretische Ebenen: Schüleraktivierung, Selbstbestimmung, Lernbegleitung und multimediales Lernen}

Der Flipped Classroom stellt sich als sehr variable Unterrichtsmethode dar, weshalb eine Basisform definiert wurde. Im Folgenden soll der Flipped Classroom lerntheoretisch verortet werden. Anknüpfungspunkte sind dabei die Überlegungen der Unterkapitel 2.1 und 2.2. Vier Ebenen bieten sich an, auf die der Flipped Classroom bezogen werden kann, die Schüleraktivierung, die Selbstbestimmung, die Lernbegleitung und das multimediale

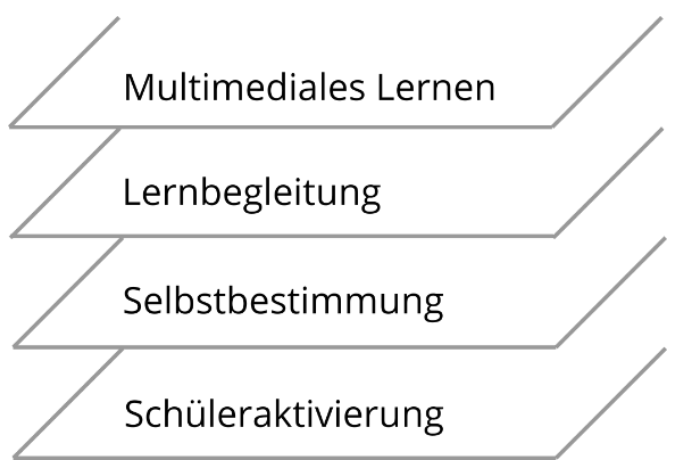
Lernen (vgl. Abb. 4).

Abb. 4. Theoretische Ebenen des Flipped Classroom

(a) Schüleraktivierung: Im Zentrum des Flipped Classroom steht nicht das Video, sondern die gewonnene Unterrichtszeit. Die Lernvideos dienen dazu, die Instruktion aus dem Unterricht auszulagern und dem Tun der Schüler mehr Raum zu geben. Daher ist der Flipped Classroom von seiner Anlage eine Form der unterrichtlichen Schüleraktivierung. Die Gefahr, das Lerngeschehen in den Clicker-Quizze und der Partner- und Gruppenarbeit passiv an sich vorbeiziehen zu lassen, ist im Unterrichtsalltag nicht ausgeschlossen, doch geringer als im traditionellen Unterricht mit einem hohen Instruktionsanteil der Lehrkraft. Die Schüleraktivierung ist lerntheoretisch die wichtigste Ebene, auf der der Flipped Classroom verortet ist. (Bishop \& Verleger 2013; Estes et al. 2014). Zahlreiche Studien belegen mit Hilfe von Fragebögen und Unterrichtsbeobachtungen, dass der Flipped Classroom den Wechsel vom lehrerzum schülerzentrierten Unterrichten fördert (Bormann 2014; Butt 2014; Deslauriers et al. 2011; Enfield 2013; Fryling et al. 2016; Johnson \& Renner 2012; Roehl et al. 2013).

(b) Selbstbestimmung: Eine weitere Ebene, auf die der Flipped Classroom bezogen werden kann, ist die Selbstbestimmungstheorie von Deci \& Ryan (2002, vgl. Kap. 2.2.1). Gemäß ihren Prinzipen kann intrinsische Motivation wachsen, wenn in Lernprozessen die Wahrnehmung von Kompetenz, Selbstbestimmung und sozialer Eingebundenheit gefördert wird. In der Basisform des Flipped Classroom ist die Selbstbestimmung noch recht rudimentär in der Selbststeuerung angelegt. Die Selbststeuerung wird auf zwei Ebenen eröffnet. Zunächst bietet das Medium der online verfügbaren Lernvideos den Schülern bei der häuslichen Vorbereitung die Möglichkeit, über Lernweg, Lernzeit und Lernort frei verfügen zu können. Sie können Videos anhalten, in ihnen zurück- und vorspringen und etwas wiederholen lassen. Durch die permanente Verfügbarkeit der Videos und die hohe Gerätemobilität der Schüler sind sie in der Lage, die Lernvideos zu beliebigen Zeiten und an beliebigen Orten zu nutzen. Des Weiteren wird für die Schüler im Unterricht durch ein binnendifferenziertes Aufgabenangebot die Möglichkeit geschaffen, über die Pflichtaufgaben hinaus frei und der Einschätzung des eigenen Leistungsniveaus entsprechend Probleme zu lösen und sich Wahlthemen zu erarbeiten.

Neben dem Prinzip der Selbststeuerung ist der Flipped Classroom durch kooperative Arbeitsformen gekennzeichnet, die die soziale Eingebundenheit der Schüler fördern. Sowohl die 
Peer Instruction der Quizphase als auch die anschließende Partner- und Gruppenarbeit bieten die Möglichkeit zum intensiven sozialen Austausch, der eng an die eigentlichen Lernhandlungen gekoppelt ist. Nach Wild \& Möller (2009) sind diese Formen der Teamarbeit und des kooperatives Lernens besonders geeignet, soziale Eingebundenheit zu fördern, welche sich positiv auf die Entwicklung intrinsischer Motivation auswirkt. So trägt die strukturelle Förderung der sozialen Eingebundenheit und der Selbststeuerung im Flipped Classroom dazu bei, was nach der Selbstbestimmungstheorie zum erfolgreichen Lernen gehört - die Vorbereitung und das Üben sowohl in eigener Regie wie in kooperativ-gegenseitiger Unterstützung (Clark et al. 2006; Deci \& Ryan 2002). Zainuddin \& Perera (2017) zeigten in einer kontrollierten Studie zur empirischen Überprüfung der selbstbestimmungstheoretischen Grundlagen des Flipped Classroom, dass Schüler im Flipped Classroom ihr Lernen kompetenzfördernder, selbstregulierter und in stärkerer sozialer Interaktion erlebten.

(c) Lernbegleitung: Der Lehrer nimmt in der Phase der Schüleraktivität eine beratende Rolle für die Schüler ein, in der er mit Hilfestellungen und Anregungen die Lernprozesse individuell, aber zurückhaltend begleiten kann. Das Lehrerhandeln im Flipped Classroom ist geprägt durch die Rolle des Lehrers als Lernbegleiter. Er gibt in dialogischer Gesprächsführung Hilfen, wenn sie gefragt sind, und Anregungen, wenn sie gebraucht werden. Durch seinen Rollenwechsel ist der Lehrer zudem besser in der Lage, individuelle Lernschwierigkeiten zu diagnostizieren und den Schüler gezielt zu unterstützen (Fahland et al. 2012; Nicolaisen 2016; Perkhofer-Czapek \& Potzmann 2016).

Genau genommen erfüllt der Lehrer im Flipped Classroom beide paradigmatischen Rollen: im Lernvideo die des Instructors, der im Instruktionsparadigma (in der Tradition von Pawlow) den Lernprozess von außen herstellt durch Anleitung, Darbietung und Erklärung, und im Unterricht als Coach, der im konstruktivistischen Paradigma (in der Tradition von Piaget) das selbstständige, vom Schüler ausgehende Lernen durch Unterstützen, Anregen und Beraten begleitet. Das Nebeneinander beider Lehrerrollen deckt sich mit der in der Neuen Lernkultur geforderten Balance von komplementären klassisch-pädagogischen und reformpädagogischen Positionen (Perkhofer-Czapek \& Potzmann 2016), wie sie in Abb. 5 skizziert ist.

Individuell ausgerichtete Lernbegleitung gehört nach Kunter \& Voss (2011) wesentlich zur konstruktiven Unterstützung von Lernenden. Damit werden auf tiefenstruktureller Ebene Grundlagen zu gelingenden Lernprozessen (vgl. Kap. 2.1) gelegt. Cornelius-White (2007) zeigt in einer groß angelegten Meta-Studie über 119 englisch- und deutschsprachige Einzelstudien, dass Variablen personenzentrierten Lehrerverhaltens (z.B. nichtdirektiv, empathisch, lernermutigend, am Lernenden orientiert, abstraktes Denken fördernd) einen überdurchschnittlich

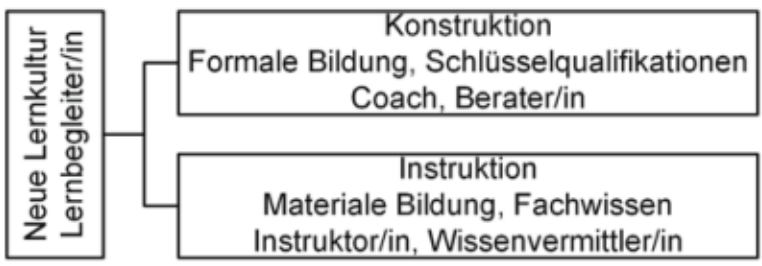

Abb. 5. Kategoriale Doppelfunktion von Lernbegleitern (nach Perkhofer-Czapek \& Potzmann 2016, S. 62) 
hohen Einfluss auf kognitive und affektive Lernergebnisse besitzen. Die Korrelation aller personenzentrierten Lehrervariablen auf die kognitiven Lernleistungen beträgt $r=.31, S D=.25$ und auf die affektiven Variablen $r=.35, S D=.20$. Der Flipped Classroom bietet den Lehrern von seiner konzeptionellen Anlage her die Gelegenheit, die Rolle des Lernbegleiters besser auszufüllen als im traditionellen Unterricht. Wenn bei Fehlern oder Hindernissen strukturiert eingegriffen werden kann und mehr Zeit für den einzelnen Schüler oder die Gruppe zur Verfügung steht, ist verständniserleichterndes Scaffolding leichter umzusetzen als im Plenum. Lernbegleitendes Lehrerhandeln im Flipped Classroom prägt demnach die Tiefenstruktur des Unterrichts und schafft Voraussetzungen für gelingende Lernprozesse.

(d) Multimediales Lernen: Eine vierte Ebene bezieht den Flipped Classroom auf die Gestaltungsrichtlinien der kognitiven Theorie des multimedialen Lernens von Mayer (2014). Der Einsatz von Lernvideos, die - wie beschrieben - in komplexerer Form auch interaktive Elemente beinhalten können, muss den Erkenntnissen Rechnung tragen, die durch die multimediale Lerntheorie zu Tage gefördert wurden. Das sind einerseits allgemeine Prinzipien der Gestaltung von digitalen Medien, wie etwa das Prinzip der dualen Kodierung (die Kombination von Bild und Text ist für das Verstehen hilfreicher als Texte allein), das Kontiguitätsprinzip (inhaltlich zusammenhängende Elemente müssen auch räumlich und zeitlich nah beieinander präsentiert sein), das Kohärenzprinzip (Eliminierung überflüssiger Elemente), das Segmentierungsprinzip (Instruktionen müssen in Sinnabschnitte unterteilt werden) oder das Modalitätsprinzip (audiovisuelle Darstellungen besitzen einen Mehrwert gegenüber TextBild-Darstellungen). Doch diese Prinzipien gelten nicht nur für Lernvideos, sondern auch für jede Art von mediengestützter Instruktion, also auch für einen klassischen Lehrervortrag unter Verwendung eines Tafelbilds, und sind deshalb nicht charakteristisch für den Flipped Classroom. Daneben werden multimedialen Lernmaterialien oft Eigenschaften zugeschrieben, für die nicht immer ausreichende Evidenzen vorhanden sind. So stellen Clark \& Feldon (2014) heraus, dass Medienvergleichsstudien, die über den Neuheitseffekt hinausgehen, keine motivationsförderlichen Wirkungen von multimedialen Lernmaterialien identifizieren können. Deshalb darf die Bedeutung der Lernvideos im Flipped Classroom nicht überschätzt werden.

Das inhärent Neue der Lernvideos gegenüber dem Lehrervortrag im Unterricht ist allerdings die oben schon angemerkte Möglichkeit, autonom und selbstständig über die Wiedergabe des Videos bestimmen zu können. Es gibt Hinweise, dass sich die Möglichkeit, Lernvideos anzuhalten, in ihnen zurück- und wieder vorzuspringen, positiv auf Lernleistungen auswirkt (Mayer \& Chandler 2001). Ob das darauf zurückzuführen ist, dass nach der Selbstbestimmungstheorie von Deci \& Ryan (2002) die vorausgehende Motivation gefördert wird oder weil nach der Cognitive Load Theory von Sweller (1994) die kognitive Belastung und damit die Informationsverarbeitung besser angepasst werden kann, ist offen. Möglicherweise spielen beide Wirkmechanismen eine Rolle. 


\subsubsection{Probleme im Flipped Classroom}

In den Erfahrungen von Dozenten und Lehrkräften, die Flipped Classroom anwenden, spiegeln sich einige praktische Probleme, die hier summarisch aufgeführt werden sollen (Goerres et al. 2015; Herreid \& Schiller 2013; Lin \& Chen 2016; Şengel 2016; Yıldrım \& Kıray 2016).

- Flipped Classroom erfordert eine Veränderung im häuslichen und schulischen Arbeiten. Deshalb nehmen die Schüler die Methode nicht selbstverständlich an, sondern müssen oft erst dafür gewonnen werden. Obwohl die häusliche Vorbereitung im Flipped Classroom niederschwelliger erscheint als die klassischen Hausaufgaben, brauchen die Schüler Verstärkung darin, sich mit den Inhalten der Lernvideos selbstverantwortlich auseinander zu setzen.

- Schüler, die unvorbereitet in den Unterricht kommen, haben einen strategischen Nachteil. Sie können nicht unmittelbar am Unterrichtsgeschehen partizipieren und sind auf die formelle oder informelle Unterstützung durch Mitschüler angewiesen.

- Die Schüler können nicht unmittelbar Verständnisfragen zum Video äußern. Alternative Formulierungen und Verstehenshilfen durch die Lehrperson oder Mitschüler können in dieser Phase nicht angeboten werden, sondern können erst im Unterricht erfolgen.

- Die Erstellung der Lernmaterialien, besonders der Lernvideos, ist zeitintensiv. Zwar können auch Fremdvideos eingesetzt werden, doch entscheiden sich viele Lehrpersonen für selbst erstellte Lernvideos.

\subsection{Flipped Classroom in der didaktischen Forschung}

Der Flipped Classroom ist keine domänenspezifische physikdidaktische Unterrichtsform - im Gegenteil, die Anwendung und empirische Untersuchung des Flipped Classroom in schulischen oder außerschulischen Lernumgebungen der Physikausbildung ist wenig verbreitet. Eine Suche in der internationalen Datenbank ERIC für wissenschaftliche Publikationen im Bildungsbereich des Institute of Education Sciences, Washington D.C., veranschaulicht die Verhältnisse. Seit 1999 gibt es 507 gelistete englischsprachige Veröffentlichungen zum Thema »flipped classroom «, von denen lediglich elf auf den Themenbereich »physics « entfallen (IES, Institute of Education Sciences, Stand: April 2018). Im Folgenden soll ein Überblick über die Forschungslage zum Flipped Classroom hinsichtlich der in Kap. 2.2 vorgestellten Konstrukte (Motivation, Interesse, Selbstkonzept und Lernleistung) gegeben werden. Anschließend wird die Relevanz aus physikdidaktischer Sicht aufgezeigt. 


\subsubsection{Bisherige Forschungsergebnisse zum Flipped Classroom}

Der Flipped Classroom ist eine Lehrform, die im Schul- wie im Hochschulbereich rezipiert wird. Vor allem in Bildungskontexten, die traditionell auf den Lehrer oder Dozenten als Instructor oder Lecturer ausgerichtet sind, erfährt der Flipped Classroom großen Zuspruch. Die meisten Veröffentlichungen zum Flipped Classroom stammen aus den USA, gefolgt von Australien, Taiwan, der Türkei, Kanada und China, wie Metastudien zum Flipped Classroom zeigen (Abeysekera \& Dawson 2015; Bishop \& Verleger 2013; Bormann 2014; Estes et al. 2014; Gillette et al. 2018; O’Flaherty \& Phillips 2015; Perselli 2016; Rahman et al. 2014; Zainuddin \& Halili 2016). Zum Einsatz des Flipped Classroom im deutschen Hochschulsystem gibt es nur vereinzelte empirische Untersuchungen (Cieliebak 2014). Meist werden nur Praxisreflexionen und Handlungsempfehlungen gegeben (Braun et al. 2012; Kenner \& Jahn 2016; Kiselka 2016; Kück 2014; Lehmann et al. 2015; Nimmerfroh 2016; Spannagel \& Freisleben-Teutscher 2016; Tolks et al. 2016). Zum aktuellen Zeitpunkt hat keine empirische Studie den Flipped Classroom im deutschsprachigen Schulsystem untersucht. Das groß angelegte Schulprojekt ,Flip your class!' begleitet die Methode derzeit an sechs Berliner Schulen und verfolgt dabei das Interesse, Handlungsempfehlungen für den Einsatz digitaler Medien im Schulunterricht geben zu können (Spannagel 2017).

Über alle Bildungsebenen und Fächer hinweg wird deutlich, dass das Hauptinteresse der internationalen Studien über den Flipped Classroom auf die Lernwirksamkeit der Methode gerichtet ist. Die Ergebnisse sind divergent. Die überwiegende Mehrheit von kontrollierten Studien in verschiedenen Disziplinen berichtet von signifikant höheren Lernleistungen im Flipped Classroom gegenüber traditionellen Lehrformen (Pearson Education 2013; Aşıksoy \& Özdaml 2016; Baepler et al. 2014; Bhagat et al. 2016; Bidwell 2014; Casasola et al. 2017; Cieliebak 2014; Clark 2015; Day \& Foley 2006; Deslauriers et al. 2011; Fautch 2015; Foldnes 2016; Galway et al. 2014; Gilboy et al. 2015; Goerres et al. 2015; González-Gómez et al. 2016; Gregorius 2017; Lee \& Wallace 2018; Lin \& Chen 2016; Love et al. 2014; MacKinnon 2015; Marcey \& Brint 2012; McLaughlin et al. 2014; Missildine et al. 2013; Pierce \& Fox 2012; Ryan \& Reid 2015; Sadaghiani 2012; Schultz et al. 2014; Şengel 2016; Sterner et al. 2017; Sun \& Wu 2016; Thai et al. 2017; Van Sickle 2016; Webster et al. 2016; Wong \& Chu 2014; Zoe et al. 2014). Somit scheinen sich die allgemeinen lerntheoretischen Überlegungen zu bestätigen, wonach die schüleraktivierende und selbstbestimmungsfördernde Art des Lernens im Flipped Classroom zu einem höheren Lernzuwachs führt. Doch eine ganze Reihe von Studien stellt auch fest, dass die Leistungen von Lernenden im Flipped Classroom sich nicht signifikant unterschieden von denen der Lernenden in traditionellem Unterricht (Bell 2015; Bishop 2013; Findlay-Thompson \& Mombourquette 2014; Gillette et al. 2018; Jensen et al. 2015; Johnson \& Renner 2012; Lape et al. 2014; Lewis et al. 2018; Marlowe 2012; Munson \& Pierce 2015; Zheng et al. 2014). Die fehlende Eindeutigkeit der Forschungsergebnisse lässt sich mit der Diversität der verwendeten Untersuchungsdesigns erklären. Offenbar macht es einen Unterschied, in welcher Form Flipped Classroom implementiert und für die Lernenden eingeführt wird. Tatsächlich lässt sich kaum eine Studie mit der anderen vergleichen, da die Formen, in denen 
Flipped Classroom umgesetzt wurde, sich deutlich voneinander unterscheiden und nicht immer detailliert beschrieben werden.

Keine besseren Lernleistungen im Vergleich zu traditionell unterrichteten Kontrollgruppen wurden erzielt, wenn die Wichtigkeit, die Lernvideos zu schauen, nicht genügend zum Ausdruck gebracht wurde (Bell 2015; Findlay-Thompson \& Mombourquette 2014), die Lernvideos auch der Kontrollgruppe zur Nachbereitung zur Verfügung standen (Munson \& Pierce 2015), die traditionelle Unterrichtsform deutlich weniger oder keine häusliche Vorbereitung vorsah und sich die Lerner im Flipped Classroom benachteiligt fühlten (Bishop 2013; Johnson \& Renner 2012), wenn Mischformen angewandt wurden, so dass Instruktionselemente in die Präsenzphase integriert wurden und sich die Lehrkräfte nicht konsequent auf die veränderte Lehrerrolle einließen (Findlay-Thompson \& Mombourquette 2014; Johnson \& Renner 2012), die Passung von Lernfortschritt und Aufgaben nicht stimmte beziehungsweise die Lehrkraft zu wenig Unterstützung gab (Bishop 2013) oder wenn die Lernvideos zu umfangreich waren - bei Bishop (2013) waren es pro Tag bis zu 17 Videos als Vorbereitung mit einer Gesamtlänge von bis zu 75 Minuten.

Neben den Unterschieden in der Methodenanwendung lassen auch Messprobleme die Sicht auf die Ergebnislage unscharf werden. So erheben einige Studien lediglich die Lernleistung nach erfolgtem Treatment durch einen Posttest, ohne sie durch einen Pretest zu kontrollieren (Day \& Foley 2006; Lape et al. 2014). Zheng et al. (2014) untersuchten die Wirkung des Flipped Classroom in einer Treatmentgruppe von $\mathrm{N}=26$ Studenten, deren abschließende Modulnoten er mit denen der Studenten des Vorjahres verglich, die traditionell unterrichtet wurden. Mit einer kleinen Stichprobe ohne Pre/Post-Design ist der Fehler, der durch die unterschiedlichen, nicht kontrollierten Schülervoraussetzungen entsteht, zu groß, um eine valide Aussage über den Methodeneffekt auf die Modulnote zu machen.

Ein Urteil über die Lernwirksamkeit des Flipped Classroom muss sich am Unterricht messen, mit dem er verglichen wird. Der Begriff »traditioneller Unterricht « ist diffus und bezeichnet in vielen Studien unterschiedliche Formen des Unterrichtens - von der klassischen Vorlesung ohne jede Aktivität der Lernenden bis zu fragend-entwickelndem Unterricht, der mit einem hohen Grad an Schüleraktivität gestaltet werden kann. Jensen et al. (2015) verglichen deshalb die Lernleistungen von College-Studenten im Flipped Classroom mit denen von Studenten in einem Parallelkurs, der von derselben Lehrkraft klassisch mit aktivierenden Methoden unterrichtet wurde. Beide Kurse wiesen in einem Vorwissenstest zu Semesterbeginn eine ausreichend hohe Gruppenhomogenität auf. Zum Vergleich der Lernleistungen wurden zwar nur die Abschlussnoten herangezogen, doch es zeigte sich, dass es hier keinen signifikanten Unterschied gab $\left(M_{f l i p}=66.6, S D=11.3 ; M_{\text {trad }}=67.9, S D=11.8 ; F(1,106)=.37, p=.55\right)$. Das bekräftigt die bereits lerntheoretisch ausgeführte Annahme, dass primär die Schüleraktivierung entscheidend für den Lernzuwachs ist. Schüler profitieren von jeder Unterrichtsform, in der sie zu kognitiver Aktivierung angeregt werden. Die bisherigen Studien deuten mehrheitlich darauf hin, dass dies im Flipped Classroom gelingt, aber selbstverständlich nicht nur dort.

Neben der Wirkung auf die kognitive Lernleistung hat der Flipped Classroom grundsätzlich auch positiven Einfluss auf affektive Lernergebnisse. Fach- und ausbildungsformübergreifend 
zeigt sich, dass die Motivation der Lernenden durch den Flipped Classroom verbessert werden kann (Aşıksoy \& Özdamlı 2016; Butt 2014; Deslauriers et al. 2011; Hao 2016; Lin \& Chen 2016; Schultz et al. 2014; Thai et al. 2017; Wong \& Chu 2014), wobei den Studien kein einheitlicher Motivationsbegriff zugrunde liegt, sondern dieser sowohl Lernmotivation, Unterrichtsengagement als auch Sachinteresse umfasst. Aber auch das Fachinteresse und die Selbsteinschätzung der Lernenden, die als übergeordneter Begriff des Selbstkonzepts verstanden werden kann, verbessern sich (Aşıksoy \& Özdaml 2016; Enfield 2013; Findlay-Thompson \& Mombourquette 2014; Hao 2016; Lin \& Chen 2016; Sun et al. 2018). Doch auch hier gibt es wie bei der Lernwirksamkeit - vereinzelt Studien, die im Vergleich zu traditionellen Lehrformen keine höheren Werte bei den Interessensvariablen feststellen. So zeigen Casasola et al. (2017) in einer Studie mit N = 547 Chemiestudenten, dass sich die über einen Fragebogen erhobenen Änderungen von Sachinteresse und Studierfähigkeiten der Treatment- und Kontrollgruppe nicht voneinander unterschieden. Jedoch verbesserten sich bei den Studenten des Flipped Classroom die Leistungen im nachfolgenden Chemiekurs signifikant um eine halbe Standardabweichung.

Trotz der positiven Korrelation von Lernleistung und Sach-/Fachinteresse (vgl. Kap. 2.2.4) gibt es in Flipped Classroom-Studien Beispiele für die Diskrepanz beider Größen - trotz besserer Notenleistungen sank das Fachinteresse. In der Evaluationsstudie von Van Sickle (2016) wiesen Studenten von vier aufeinander folgenden College-Mathematikkursen im Flipped Classroom signifikant niedrigere Fachinteressenswerte auf als die Studenten von sechs Kursen gleichen Inhalts im traditionellen Format $\left(M_{\text {flip }}=3.95, S D=1.06 ; M_{\text {trad }}=4.34, S D=.074\right.$; $p=.04$ ). Dagegen sind die Abschlussnoten in den Flipped Classroom-Kursen signifikant besser als in den Vergleichskursen $\left(M_{\text {flip }}=77.2, S D=10.0 ; M_{\text {trad }}=70.5, S D=19.4 ; p=.0143\right)$. In allen Kursen unterrichtete dieselbe Lehrkraft. Die Diskrepanz zwischen Leistung und Fachinteresse mag der Art geschuldet sein, wie die Lehrkraft Flipped Classroom in den Kursen implementiert hat. In der Kursevaluation erhielt sie signifikant schlechtere Werte in den Items: »The instructor encouraged my interest in the course. « und »Overall, the instructor effectively facilitated my learning. «Doch scheint die negative Einschätzung über den Flipped Classroom-Kurs und seine Lehrkraft der höheren Lernwirksamkeit keinen Abbruch getan zu haben. Dieses Beispiel macht deutlich, dass die Arbeitsweise im Flipped Classroom tatsächlich positive Wirkung auf die kognitiven Lernprozesse besitzt, selbst wenn diese nur über die extrinsische Motivation der Kursanforderungen und nicht über eine gesteigerte intrinsische Motivation vermittelt werden.

Ein weiterer konzeptioneller Aspekt des Flipped Classroom soll noch mit Hilfe empirischer Studien betrachtet werden, das Thema der Lehrerunterstützung. In vielen Studien gaben die Lernenden an, dass die Lernzeit in der Präsenzphase effektiver genutzt wurde, weil sie stärker mit dem Lehrer oder Dozenten interagiert haben (Butt 2014; Fulton 2012; Greenberg et al. 2011; Hao 2016; Ryan \& Reid 2015; Schultz et al. 2014). Die konzeptuell veränderte Rolle des Lehrers hin zum Lernbegleiter scheint auch für die Schüler wahrnehmbar zu sein. Davon abweichend stellten Sun \& Wu (2016) fest, dass es bei der Qualität der Lehrer-Schüler-Interaktionen im Flipped Classroom keinen signifikanten Unterschied zu den Angaben der Schüler 
im normalen Unterrichtsformat gab $\left(M_{\text {flip }}=4.46, S D=.60 ; M_{\text {trad }}=4.41, S D=.75 ; t(154)=.44\right.$, $\mathrm{p}=.66$ ), obschon auf je drei Schülergruppen ein Betreuer als Unterstützung kam. Einschränkend muss jedoch angemerkt werden, dass die Betreuer im Flipped Classroom angewiesen wurden, sich parallel Notizen zu den Gruppen und ihren Mitgliedern zu machen, um im Anschluss Gruppen- und Einzelpreise für die besten Leistungen zu vergeben. Mit diesem kompetitiven und leistungskontrollierenden Element ist es denkbar, dass die Hemmschwelle für die Schüler höher war, die Betreuer um Hilfe oder Unterstützung zu bitten, weshalb keine erhöhte Schüler-Lehrer-Interaktion zu messen waren. Dass dennoch die Abschlussleistungen der Schüler im Flipped Classroom deutlich besser waren als im traditionellen Unterricht $\left(M_{\text {flip }}=69.09, S D=13.28 ; M_{\text {trad }}=62.58, S D=15.31 ; F(1,153)=9.70 ; p<.01\right)$, mag ebenfalls durch den zusätzlichen Leistungsanreiz beeinflusst worden sein. In der oben erwähnten Evaluationsstudie von Van Sickle (2016) sind die Rückmeldungen der Schüler für die Lehrerunterstützung im Flipped Classroom sogar schlechter ausgefallen als im traditionellen Unterricht.

\subsubsection{Physikdidaktisches Interesse am Flipped Classroom}

Die europäische Physikdidaktik hat die Entwicklung des Flipped Classroom bisher noch nicht begleitet. Überhaupt ist, wie im Vorkapitel angemerkt, der Flipped Classroom als Unterrichtsmethode nicht oder noch nicht sehr etabliert im physikbezogenen Bildungskontext. Generell besitzt der Flipped Classroom im deutschen Hochschulsystem, vor allem aber im deutschen Schulsystem noch Exotenstatus. Deshalb soll im Folgenden die Chance für den schulischen Physikunterricht aufgezeigt werden, die sich in dieser Methode verbirgt und zu den Forschungsfragen führt.

Die Physikdidaktik hat ein Interesse, lernwirksame Merkmale naturwissenschaftlichen Unterrichts zu identifizieren und ihre Förderung zu untersuchen. Die fachübergreifenden Merkmale der Unterrichtsqualität, welche in Kap. 2.1 und 2.2 erläutert werden, gelten selbstverständlich auch für den Physikunterricht und sind auch dort für das Lernen relevant (Neumann et al. 2012). Darüber hinaus gibt es in den Naturwissenschaften spezifische Merkmale der Unterrichtsqualität, welche nur ihnen eigen sind. Zu diesen fachspezifisch schüleraktivierenden und Scientific Literacy fördernden Tätigkeiten gehört der forschend-entdeckende Unterricht, der in der Fachdidaktik als besonders physikauthentisch gilt (Höttecke \& Rieß 2015). Ob der Flipped Classroom forschend-entdeckenden Unterricht unterstützt oder nicht, hängt allein von den unterrichtlichen Aktivitäten ab, die durch die Lernvideos vorentlastet werden. Wenn die Lernvideos auf Unterrichtshandlungen vorbereiten, in denen prozedurales Wissen (Wissen über Verfahren und Methoden zur Generierung naturwissenschaftlicher Erkenntnisse) oder epistemisches Wissen (erkenntnistheoretisches Metawissen über die Art und den Ursprung naturwissenschaftlichen Fachwissens, also über die Natur der Wissenschaft) gefördert werden, findet der Flipped Classroom im forschend-entdeckenden Naturwissenschaftsunterricht seinen Platz wie andere Methoden potentiell auch. Allerdings gibt es bisher keinen Nachweis, dass der Flipped Classroom mit dieser Intention eingesetzt wurde. Die inhaltliche Ausrichtung der Methode, also die Gestaltung der Lernvideos und der Unterrichtsaktivitäten, 
lag bisher in der Förderung, Festigung und Vertiefung von deklarativem Fachwissen in engerem Sinn.

Was den naturwissenschaftlichen Unterricht im Allgemeinen und den Physikunterricht im Besonderen gegenüber anderen Fächern abgrenzt, ist die Rolle des Experiments. Das Experimentieren erfüllt aus konstruktivistischer Sicht eine Vielzahl von Funktionen für den kognitiven Wissensaufbau der Schüler und nimmt daher eine zentrale Stellung im Physikunterricht ein (Tesch \& Duit 2004). Es möchte zugleich auch Interesse und Motivation wecken und damit den affektiven Lernbereich ansprechen. Wo der Flipped Classroom das Experiment organisch integriert, kann diese Methode auch im Physikunterricht sinnvoll eingesetzt werden. Wo dauerhaft weder im Lernvideo noch in der Unterrichtsaktivität auf reale Experimente zurückgegriffen wird, widerspricht die Anwendung des Flipped Classroom einer wichtigen fachimmanenten Zieldimension, weil Theorie und Praxis nicht miteinander verbunden werden. Von den vorliegenden Studien zur Anwendung des Flipped Classroom in der Physikausbildung (Aşıksoy \& Özdamlı 2016; Atwa et al. 2017; Bates \& Galloway 2012; Deslauriers et al. 2011; Sadaghiani 2012; Şengel 2016; Sun \& Wu 2016; Sun et al. 2017; Winter 2013; Zoe et al. 2014) wurden keine Realexperimente in die Lernvideos integriert. Lediglich Şengel (2016) erwähnt, dass an geeigneten Stellen YouTube-Videos mit Simulationen oder einfachen Freihandversuchen zur ergänzenden Erklärung verwendet wurden. Ansonsten wurden Vorlesungsmitschnitte, audiokommentierte Bildschirmaufzeichnungen (sogenannte Voice-over-Screencasts) von Folienpräsentationen oder videografierte Paper-Pencil- oder WhiteboardHerleitungen verwendet, also digitale »Kreidephysik «. ${ }^{5}$ Auf die Lernleistungen hatte diese Art des Physiklernens keine negative Auswirkung - sämtliche erwähnten Studien berichten von verbesserten Lernleistungen durch den Flipped Classroom. Lediglich Winter (2013) misst nach einem fünfwöchigen Treatment in einem College keine Leistungsunterschiede zur Vergleichsgruppe im traditionellen Physikunterricht.

Aussagen über vergleichende affektive Lernergebnisse finden sich in keiner physikbezogenen Studie. Aşıksoy \& Özdamlı (2016) maßen in ihrer Studie in einem universitären Physikeinführungskurs neben einem erhöhten Lernzuwachs auch eine signifikante Erhöhung von Motivation und Selbstwirksamkeit bei Studierenden im Flipped Classroom nach einem achtwöchigen Treatment, doch wurden in der Kontrollgruppe Motivation und Selbstwirksamkeit nicht gemessen, so dass keine vergleichende Aussagen möglich sind.

Zusammenfassung: Der Flipped Classroom hat auf internationaler Ebene in den letzten Jahren zunehmende Aufmerksamkeit in der empirischen Bildungs- und Didaktikforschung erlangt. Ihm wurden mehrheitlich positive Effekte in kognitiven wie affektiven Lernbereichen bescheinigt. In den wenigen physikbezogenen Studien steht, wie bei den meisten anderen auch, generell der Einfluss auf die Lernleistung im Vordergrund. Die Wirkungen des Flipped

5 Davon abgehoben sind Praktikumskurse im Hochschulbereich, die seit jeher so konzipiert sind, dass die Studierenden die Theorie zu den Experimenten häuslich erarbeiten müssen, bevor sie diese im Praktikum durchführen. Wenn den Studierenden statt Skripten allein zusätzlich nun erklärende Videos angeboten werden, ist das ein didaktischer Mehrwert, aber kein neues Lehrkonzept, auch wenn wegen der Videos oft von Flipped Classroom gesprochen wird (z.B. Robinson et al. 2015). 
Classroom auf kognitive wie auch affektive Lernergebnisse in einem von realen Experimenten gekennzeichneten schulischen Physikunterricht des deutschen Bildungssystems wurden bislang noch nicht untersucht. Hier drängt sich die Frage auf, ob die Anwendung des Flipped Classroom im schulischen Physikunterricht einen positiven Beitrag für das fachliche Lernergebnis, aber auch die Entwicklung der Motivation und des Interesses leisten kann. 


\section{Forschungsfragen und Hypothesen}

Die vorliegende Studie möchte untersuchen, wie die Unterrichtsmethode Flipped Classroom im schulischen Physikunterricht der Oberstufe wirkt. Deshalb stehen die kognitiven und affektiven Lernergebnisse im Fokus, die Schüler in dieser Unterrichtsmethode im Vergleich zu Schülern erzielen, welche traditionell unterrichtet werden. Da Motivation, Interesse und Selbstkonzept $^{6}$ keine statischen Persönlichkeitszüge sind, sondern interdependente Lernmerkmale, die sich - wie in Kap. 2.2 gezeigt - durch äußere Anreize verändern können, soll geprüft werden, ob bereits eine mehrwöchige Intervention durch Flipped Classroom mesoskopisch Einflüsse besitzt.

Wie in Kap. 2.1 gesehen, ist die kognitive Aktivierung maßgeblich für das fachliche Lernergebnis verantwortlich (Hake 1998; Kunter \& Voss 2011). Die intrinsische Motivation, sich mit Physik zu beschäftigen, ist direkter Ausdruck eines individuellen Interesses, welches sich nach Hidi \& Renninger (2006) ausgehend von evoziertem situationalen Interesse sukzessiv stabilisieren kann. Motivation ist ein zentraler Bedingungsfaktor schulischer Lernleistung (Krapp 2003) und ist - wie ein positives Selbstkonzept - eine eigenständige Zieldimension schulischen Lernens (Möller \& Trautwein 2009; Stiensmeier-Pelster \& Schöne 2008). Das Fachinteresse ist eine mehrfaktorielle Größe und beeinflusst - wie das individuelle Sachinteresse beziehungsweise die intrinsische Motivation - das kognitive Lernverhalten. Da die Variablen Lernleistung, intrinsische Motivation, Fachinteresse und Selbstkonzept in einem sehr engen Wechselverhältnis stehen, ist es in einer Studie zum Flipped Classroom angezeigt, die gemeinsame Entwicklung dieser Variablen und mögliche Moderatoreffekte zu untersuchen. Insgesamt werden drei Forschungsfragen formuliert, die in sechs begründeten Hypothesen münden.

Forschungsfrage 1. Wie beeinflusst der Flipped Classroom im schulischen Physikunterricht die Lernleistung, die Motivation zur Physik, das Interesse am Fach Physik und das physikbezogene Selbstkonzept von Schülern im Vergleich zum traditionellen Physikunterricht?

Hypothese 1a. Der Flipped Classroom steigert die Lernleistung im Vergleich zu traditionellem Physikunterricht.

Begründung: Anknüpfend an die Mehrheit an internationalen Studien, die eine höhere Lernwirksamkeit des Flipped Classroom in anderen Fächern und Bildungssystemen festgestellt hat (Bishop \& Verleger 2013; Bormann 2014; Casasola et al. 2017; Fautch 2015; Gregorius 2017; O'Flaherty \& Phillips 2015; Şengel 2016), ist die Annahme, dass die Methode auch im Physikunterricht an Schulen im deutschen Bildungssystem die Lernleistung von Schülern

6 In Kap. 3-7 sind alle Variablen, die im Rahmen der Studie erhoben und ausgewertet wurden, der besseren Lesbarkeit halber kursiv gesetzt. 
verbessert, naheliegend. Lernleistung wird hier verstanden als Zuwachs an deklarativem Wissen. Auf der anderen Seite stellt eine beträchtliche Zahl von Studien keine höheren Lernleistungen im Flipped Classroom fest (Bell 2015; Jensen et al. 2015; Lape et al. 2014; Munson \& Pierce 2015). Ausgehend von den theoretischen Überlegungen lässt sich im Flipped Classroom eine erhöhte kognitive Aktivierung und eine höhere Lernleistung erwarten - bei vergleichbaren Bedingungen und Anforderungen in einer Kontrollgruppe. Begründet wird diese Annahme durch die lernförderlichen Elemente des Flipped Classroom - die Schülerzentrierung, das kooperative Lernen, die intensive Nutzung der Unterrichtszeit für Lernaufgaben, das lernbegleitende Lehrerverhalten sowie die mediale Unterstützung durch die Lernvideos. Eine empirische Bestätigung dieser Annahme für den deutschen Schulunterricht im Allgemeinen und für den schulischen Physikunterricht im Besondern steht aus.

Hypothese 1b. Der Flipped Classroom hat einen positiven Effekt auf die Entwicklung der affektiven Lernmerkmale Motivation, Fachinteresse und Selbstkonzept im Vergleich zum traditionellen Physikunterricht.

Begründung: Die intrinsische Motivation zur Beschäftigung mit physikalischen Themen schulisch wie außerschulisch - ist Ausdruck eines Sachinteresses. Dieses kann nach dem Vier-Phasen-Modell von Hidi \& Renninger (2006) verschiedene Grade an Stabilität besitzen, also situational ausgelöst werden und sich zunehmend zu individuellem Interesse verfestigen. Parallel dazu wandelt sich aktuelle in habituelle Motivation. Nach der Selbstbestimmungstheorie von Deci \& Ryan (2002) kann intrinsische Motivation gefördert werden, wenn Rahmenbedingungen für das Erleben von Kompetenz, Selbstbestimmung und sozialer Eingebundenheit geschaffen werden. Der Flipped Classroom spricht diese Bedürfnisse an, wenn Schüler selbstgesteuert die Lerninhalte der Videos rezipieren und in offenen, kooperativen Lernformen unter defensiver Begleitung der Lehrperson physikrelevante Fähigkeiten und Wissensstrukturen ausbauen.

In anderen Fachdisziplinen konnten das Selbstkonzept (Aşıksoy \& Özdamlı 2016; Enfield 2013; Findlay-Thompson \& Mombourquette 2014) und die Motivation (Butt 2014; Deslauriers et al. 2011; Hao 2016) gefördert werden, wobei der motivationale Neuheitseffekt von innovativen, technologiebasierten Lehrmethoden zu berücksichtigen ist (Clark 1983). Hoffmann et al. (1998) zeigen, dass sich Unterrichtsmerkmale wie Unterrichtsklima, Unterrichtsmanagement und Schülerbeteiligung auf das durch den Physikunterricht induzierte Sachinteresse auswirken können. Dieselben Autoren zeigen jedoch, dass das Fachinteresse an Physik im Laufe der Schulzeit kontinuierlich sinkt, was sich schon innerhalb von Monaten (Daniels 2008) oder gar Wochen (Weber 2003; Winkelmann 2013) nachweisen lässt. Auch wenn der Flipped Classroom keinen der bisher als wirkungsvoll nachgewiesenen Mechanismen verwendet, diese Fachinteressensdegeneration aufzuhalten (etwa durch Verstärkung von inhaltlicher Kontextualisierung und Alltagsbezügen, vgl. Häussler \& Hoffmann 1995; Walper 2017), liegt die Vermutung nahe, dass das Potenzial des Flipped Classroom, Motivation, Fachinteresse und das Selbstkonzept zu stärken, auch im Physikunterricht wirk- 
sam werden kann. Mutmaßlich, aber noch unbestätigt lassen sich die bisherigen Erwartungen an den Flipped Classroom stellen, wenn diese Methode im gymnasialen Physikunterricht eingesetzt wird.

Forschungsfrage 2: Welchen Einfluss haben das Leistungsniveau und das Geschlecht auf die Lernleistung, die Motivation, das Fachinteresse und das Selbstkonzept im Flipped Classroom?

Hypothese 2a. Das Leistungsniveau im Fach Physik beeinflusst nicht die Wirkung des Flipped Classroom auf die Lernleistung, die Motivation, das Fachinteresse und das Selbstkonzept.

Begründung: Das Leistungsniveau im Fach Physik wird hier verstanden als die Summe der kognitiven Fähigkeiten im Fach Physik. Es wird abgebildet in der Notenleistung, welche als Zeugnisnote operationalisiert wird. Sie besitzt Einfluss auf zukünftige Lernleistungen im Fach Physik, also auch auf die Lernleistung im Flipped Classroom. Die Frage ist, ob Flipped Classroom eine Methode ist, von der physikbegabte und leistungsstarke Schüler mehr profitieren als weniger physikbegabte und leistungsstarke - oder andersherum, ob vielleicht gerade leistungsschwache Schüler im Flipped Classroom einen Nutzen hinsichtlich der kognitiven und affektiven Lernerfolge haben. Beides ist nicht von der Hand zu weisen. Die größere Selbststeuerung und höhere innere Differenzierung im Flipped Classroom kann einerseits besonders den Schülern in der Leistungsspitze zugutekommen. Im traditionellen Unterricht, der in der berechtigten Sorge um die leistungsschwachen Schüler oft kleinschrittig und lernsynchron gestaltet ist, bleiben diese Schüler oft unter ihren Möglichkeiten. Innere Differenzierung gilt als ein Aspekt der Förderung von hochbegabten Schülern (Rost et al. 2006). Zum anderen ist es aber auch denkbar, dass gerade leistungsschwache Physikschüler vom Flipped Classroom profitieren, da sie einen größeren Nutzen von der kollaborativen Unterstützung durch Mitschüler in der Präsenzphase haben als leistungsstärkere Physikschüler, worauf Ergebnisse von Marlowe (2012) und Ryan \& Reid (2015) hindeuten. Lo \& Hew (2017) legen für das Fach Mathematik nahe, dass besonders die Ränder des Schülerleistungsspektrums, also sowohl leistungsschwache als auch leistungsstarke Schüler, vom Flipped Classroom profitieren. In einer Hochschulstudie von Goerres et al. (2015) zum Flipped Classroom in der Politikwissenschaft gab es keine Interaktion mit dem Leistungsvermögen. Die vorliegende Studie geht deshalb von der Nullhypothese aus, dass das Leistungsniveau im Fach Physik, das sich in der Notenleistung ausdrückt, den Effekt des Flipped Classroom nicht moderiert.

Hypothese 2b. Das Geschlecht beeinflusst nicht die Wirkung des Flipped Classroom auf die Lernleistung, die Motivation, das Fachinteresse und das Selbstkonzept.

Begründung: Die Rolle des Geschlechts im Physikunterricht ist von großem fachdidaktischen Interesse. Während sich in den Schulnoten im Fach Physik kein Geschlechterunterschied nachweisen lässt (Krapp \& Prenzel 2011), stellt die PISA-Erhebungsrunde 2015 fest, dass erstmals sowohl im OECD-Durchschnitt als auch in Deutschland die naturwissenschaftliche 
Kompetenz von Jungen signifikant besser war als von Mädchen (Schiepe-Tiska et al. 2016). Auch die motivational-affektiven Lernmerkmale zeigen deutliche Unterschiede zugunsten der Jungen. Mädchen besitzen signifikant niedrigere Sach- und Fachinteressenswerte sowie ein niedrigeres Selbstkonzept (Herbst et al. 2016; Häussler \& Hoffmann 1995; Krapp \& Prenzel 2011; Wolters \& Pintrich 1998). Der Flipped Classroom ist keine inhaltsabhängige Methode und nimmt keine Elemente auf, die eine besondere Interessens- und Motivationsförderung von Mädchen in den Blick nimmt. Deshalb ist zunächst von der Nullhypothese auszugehen, dass es keine Interaktion von Geschlecht und Methode gibt. Der Flipped Classroom sollte für Mädchen genauso wirksam sein wie für Jungen. Dennoch besitzt diese Fragestellung einen Wert. Schultz et al. (2014) zeigten, dass Jungen im Flipped Classroom im Chemieunterricht der 11. Jahrgangsstufe signifikant bessere Leistungen als Mädchen erzielten. Deshalb soll auch in dieser Studie der Frage nachgegangen werden, ob das Geschlecht einen Unterschied macht.

Auch wenn das Selbstkonzept digitale Kompetenz von Jungen und ihre Begeisterung für digitale Geräte höher sind als die von Mädchen, besitzen sie keine besseren internettechnologischen Grundlagenfähigkeiten (Christoph et al. 2015). Für die Nutzung von Online-Lernvideos bedarf es keiner Fähigkeiten höherer Ordnung, weshalb auch aus Sicht der digitalen Kompetenz die Nullhypothese naheliegt.

Die letzte Forschungsfrage zielt darauf ab, welche mittelfristigen Veränderungen die Schüler durch den Flipped Classroom in den lernbegleitenden Kontexten erfahren, die hier Stützfaktoren genannt werden.

Forschungsfrage 3: Wie verändert der Flipped Classroom Stützfaktoren (Lehrerunterstützung, Hausaufgabendauer und -disziplin) des Unterrichts?

Hypothese 3a. Der Flipped Classroom erhöht die von den Schülern wahrgenommene Lehrerunterstützung.

Begründung: Eine Reihe internationaler Evaluationsstudien zeigt, dass Lernende sich im Flipped Classroom besser unterstützt fühlen als in traditionellen Lehrformaten (Butt 2014; Hao 2016; Schultz et al. 2014), trotz Studien mit abweichenden Ergebnissen (Sun \& Wu 2016; Van Sickle 2016). Es ist anzunehmen, dass auch im schulischen Physikunterricht der Rollenwechsel im Lehrerverhalten durch den Flipped Classroom für die Schüler wahrnehmbar ist, wenn das Lehrerhandeln stark auf die adaptive Begleitung und das Scaffolding der Schülergruppen und ihrer Teilnehmer ausgerichtet ist. In den genannten Studien ist die von Van Sickle (2016) jedoch die einzige, in der die Lehrperson sowohl Kontroll- als auch Treatmentgruppe unterrichtete. Offen ist die Frage, ob eine kontrollierte Studie im Physikunterricht, in der jede teilnehmende Lehrperson eine Kontroll- und Treatmentklasse unterrichtet, zu einem anderen Ergebnis kommt.

Das Lehrerverhalten ist eng an die Lehrerpersönlichkeit und die bisherigen Lehrroutinen geknüpft. Es lassen sich Lehrerpersönlichkeiten vorstellen, deren Begleitung im Flipped 
Classroom sehr eng, direktiv und kontrollierend ist. Das würde der Intention einer Unterrichtsmethode entgegenlaufen, die Selbstbestimmung, Kompetenz und soziale Eingebundenheit fördern will. Der Flipped Classroom ist auch für die Lehrperson kein methodischer Selbstläufer, und es braucht in einer kontrollierten Studie Einweisung und Leitlinien für die Betreuung der Schüler. Die Lehrperson ist grundsätzlich immer ein Faktor, der maßgeblich die kognitiven und affektiven Lernerfolge der Schüler beeinflusst. Im Rahmen dieser Studie über den Flipped Classroom wird er angemessen zu berücksichtigen sein, auch wenn das Hauptaugenmerk nicht auf der Rolle der Lehrperson liegt.

Hypothese 3b. Bei vergleichbarem Workload investieren die Schüler im Flipped Classroom durchschnittlich nicht mehr Zeit in die häusliche Vorbereitung für eine Unterrichtsstunde, erledigen sie aber häufiger.

Begründung: Es ist anzunehmen, dass das vorbereitende Lernen mit Videos im Flipped Classroom für Jugendliche niederschwelliger ist als das nachbereitende Aufgabenrechnen und Problemlösen im traditionellen Unterricht. Wenn dem so ist, sollten die Schüler im Flipped Classroom ihre häusliche Vorbereitung mit einer größeren Disziplin erledigen als die Schüler im traditionellen Unterricht. Voraussetzung dabei ist, dass eine vergleichbare Zeit für die häusliche Aktivität in Kontroll- und Treatmentgruppe vorgesehen ist. Wenn der Aufwand für die eine oder andere Gruppe wesentlich größer ist (Bishop 2013; Johnson \& Renner 2012), führt das zu einer Verzerrung, so dass die Frage nach der Hausaufgabendisziplin nicht mehr vergleichend beantwortet werden kann. Der Workload für beide Gruppen ist eine Frage der Planung. Ist die durchschnittliche Hausaufgabendauer in beiden Gruppen ähnlich, ist von vergleichbarem Workload auszugehen. 


\section{Inhalte und Lernmaterialien}

Die Unterrichtsreihe, auf die Flipped Classroom im Physikunterricht angewandt werden sollte, musste mehrere Kriterien erfüllen. Zum einen sollte sie für die Schüler eine höhere kognitive Herausforderung bieten als andere, so dass Aktivwerden und intensives Üben noch wichtiger sind. Nur hier kann sich zeigen, ob die Wahl des Unterrichtsformats einen Unterschied für den kognitiven Lernerfolg macht oder nicht. Es gibt Unterrichtsreihen, in denen es erfahrungsgemäß leichter fällt, den Instruktionsanteil der Lehrkraft niedrig und den Umfang der Schüleraktivität hoch zu halten. Doch in der Oberstufe zeigt sich, dass die Stoffdichte und das Anforderungsniveau im Physikunterricht deutlich höher sind als in der Mittelstufe. Der Instruktionsanteil der Lehrkräfte steigt und die Herausforderung wird größer, die Aktivität der Schüler aufrecht zu halten. Deshalb fokussierte sich die Suche auf eine Unterrichtsreihe in der Oberstufe. Des Weiteren sollte die Unterrichtsreihe hinreichend umfangreich sein, so dass sich der motivationale Neuheitseffekt einer neuen, technikbasierten Methode abnutzt. Clark (1983) spricht von acht Wochen Treatment, nach denen man keinen nennenswerten Effekt, der von Neuheit herrührt, registrieren kann. Und schließlich muss sie so in die Jahresplanung einzubetten sein, dass sie von keiner regulären schulischen Leistungserhebung unterbrochen wird. Wenn sich die Schüler während des Treatments auf eine Klausur vorbereiten müssten, wäre dieses zeitweilige, extrinsisch motivierte Lernengagement gegenüber der Vergleichsgruppe nicht zu kontrollieren. Aus diesen Gründen fiel die Wahl auf die beiden sequentiellen Themenbereiche Elektromagnetische Induktion und Elektromagnetischer Schwingkreis im zweiten Halbjahr der 11. Jahrgangsstufe, die insgesamt etwa acht Wochen Schulunterricht in Anspruch nehmen. Der zeitliche Rahmen machte es notwendig, Ferienblöcke einzuplanen. Zwei Ferienblöcke von jeweils einer und zwei Wochen unterbrachen die Unterrichtsreihe, so dass sich die untersuchte Unterrichtsreihe für alle Schüler auf insgesamt fast drei Monate ausdehnte.

Um die Forschungsfragen zu beantworten, war ein Gruppenvergleich von Schülern, die im Flipped Classroom (Treatmentgruppe) unterrichtet wurden, mit Schülern, die traditionell unterrichtet wurden (Kontrollgruppe), notwendig. Im Folgenden werden zunächst die inhaltlichen Gemeinsamkeiten beider Gruppen beschrieben: die Lerninhalte, die zentralen Experimente und ein Pool an Übungsaufgaben. Anschließend wird ein Überblick über die Lernvideos gegeben, die ausschließlich in der Treatmentgruppe zum Einsatz kamen.

\subsection{Inhalte der Unterrichtsreihe »Elektromagnetische Induktion und elektromagnetischer Schwingkreis«}

Die Grundlagen zum Thema Induktion wurden bereits in der 9. Jahrgangsstufe gelegt und gemäß Lehrplan in der 11. Jahrgangsstufe wieder aufgegriffen und vertieft (BStMUK, 
Bayerisches Staatsministerium für Unterricht und Kultus 2004). Zuvor hatten die Schüler statische elektrische und magnetische Felder quantitativ betrachten gelernt. Daran schloss sich die Bewegung von Teilchen in Feldern an, so dass die Lorentzkraft als Wirkung auf bewegte, geladene Teilchen in Magnetfeldern die Brücke zur Unterrichtsreihe über die elektromagnetische Induktion bildet.

Die nachfolgende Übersicht (Tab. 1) gibt die Sequenzierung, die zentralen Inhalte und die Experimente der Unterrichtsreihe wieder. Die Übersicht wurde vom Autor gemeinsam mit den anderen an der Studie teilnehmenden Lehrkräften erarbeitet und diente zur Unterrichtsplanung. Alle Lehrkräfte besaßen mindestens fünf Jahre Berufserfahrung, hatten zuvor mindestens drei Mal die 11. Jahrgangsstufe unterrichtet und Abiturerfahrung im Fach Physik. Folglich waren alle Lehrkräfte in Theorie und Praxis hinreichend mit den Vorgaben des Lehrplans und den Erwartungen vertraut, die an die Schüler gestellt werden, die sich für Physik als Abiturfach entscheiden sollten. Die Übersicht wurde so gestaltet, dass die Lehrkräfte den Unterricht in größtmöglicher inhaltlicher Parallelität gestalten konnten. Trotzdem sollte eine maßvolle pädagogisch-didaktische Freiheit gewährt werden, um auf die Anforderungen ihrer spezifischen Lerngruppe eingehen zu können.

Tab. 1. Übersicht über die Lerninhalte der Unterrichtsreihe

\begin{tabular}{|c|c|c|}
\hline Lehrplan & Einheiten & Experimente \\
\hline \multicolumn{3}{|l|}{ Induktion (4 Wochen) } \\
\hline $\begin{array}{l}\text { Induktion im bewegten und im ru- } \\
\text { henden Leiter }\end{array}$ & $\begin{array}{l}\text { Induktion durch Bewegung und } \\
\text { Magnetfeldänderung, Rechte- } \\
\text { Hand-Regel: } \\
\text { - Bewegung eines geraden } \\
\quad \text { Leiters im konstanten Mag- } \\
\text { netfeld; } \\
\text { Bewegung eines Leiterrah- } \\
\text { mens, dann einer Spule im } \\
\text { Magnetfeld } \\
\text { - Zeitliche Änderung des Mag- } \\
\text { netfelds (Transformator) }\end{array}$ & $\begin{array}{l}\text { - Leiterschaukel } \\
\text { - Relativbewegungen von Spule } \\
\text { und Magnet } \\
\text { - Transformatorprinzip }\end{array}$ \\
\hline $\begin{array}{l}\text { Magnetischer Fluss und Indukti- } \\
\text { onsgesetz }\end{array}$ & $\begin{array}{l}\text { Induktive Herleitung von } \\
U_{i}=-n \dot{\Phi} \\
\text { (Vorgriff auf Lenzsche Regel) }\end{array}$ & $\begin{array}{l}\text { Experimentelle Bestätigung } \\
\text { (z.B. Kabel mit v = const. durch } \\
\text { das Feld eines Hufeisenmagne- } \\
\text { ten ziehen und die Spannung } \\
\text { messen) }\end{array}$ \\
\hline $\begin{array}{l}\text { Energieerhaltung und Lenzsche } \\
\text { Regel }\end{array}$ & Lenzsche Regel & $\begin{array}{l}\text { - Thomsonscher Ringversuch } \\
\text { - Wirbelstrombremse } \\
\text { - Magnet in Fallrohr }\end{array}$ \\
\hline $\begin{array}{l}\text { Erzeugung sinusförmiger Wechsel- } \\
\text { spannung }\end{array}$ & $\begin{array}{l}\text { Generatorprinzip, } \\
A(t)=A_{0} \cos (\omega t) \\
U_{\text {int }}(t)=U_{0} \sin (\omega t) \\
\text { Effektivleistung } \\
U_{\text {eff }}=U_{\max } / \sqrt{2}\end{array}$ & $\begin{array}{l}\text { - Generator (Hufeisenmagnet, } \\
\text { Polschuhe, Leiterrahmen) }\end{array}$ \\
\hline
\end{tabular}




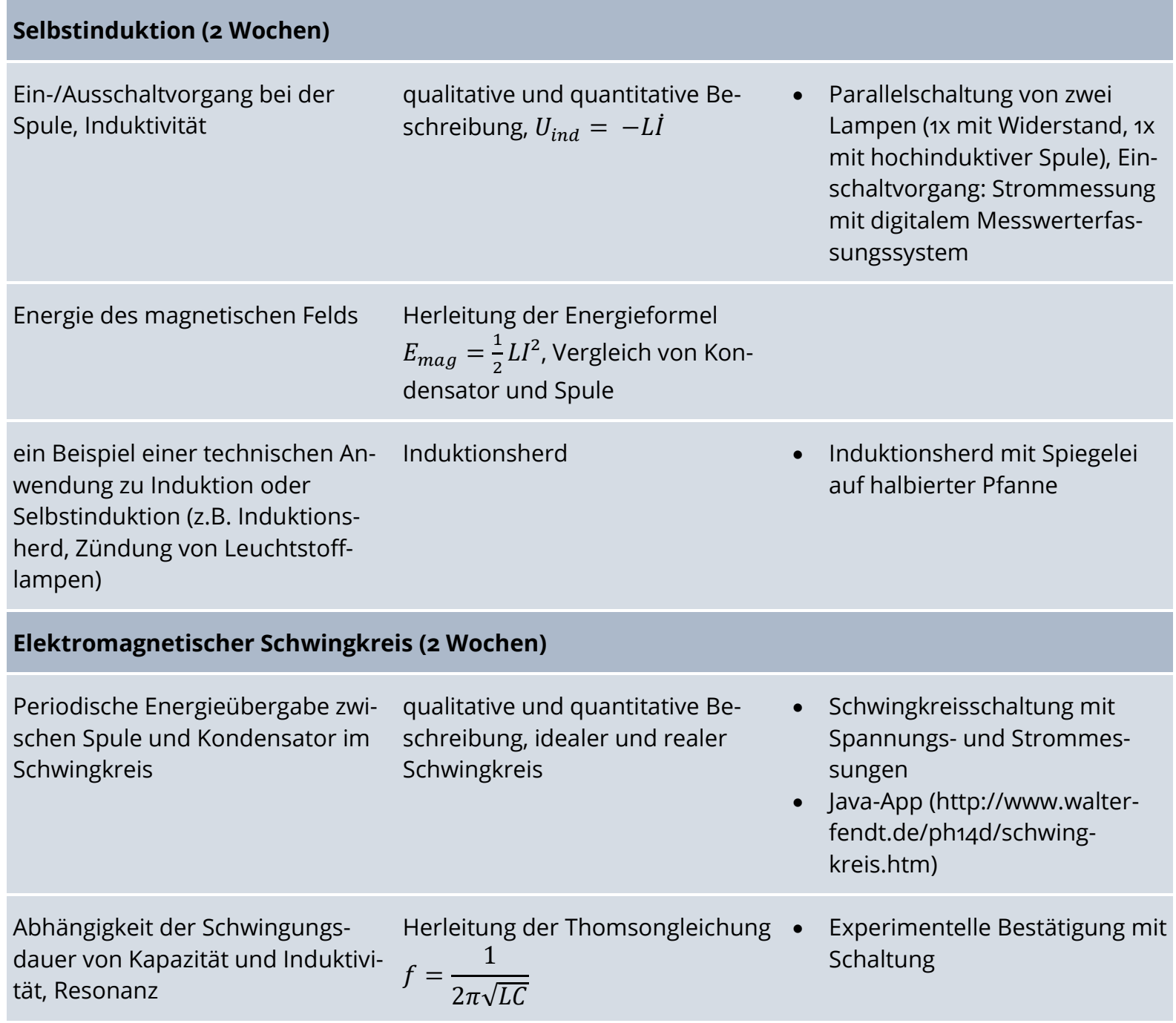

Sämtliche Versuche wurden für die Kontrollgruppe als Demonstrationsversuche durch die Lehrkraft durchgeführt. Die Durchführung von Schülerexperimenten ist in der täglichen Praxis des Physikunterrichts der Oberstufe nur eingeschränkt möglich und wurde in dieser Studie gänzlich außer Acht gelassen, um der Kontrollgruppe keinen Vorteil zu verschaffen. Die Experimente, die im Unterricht der Kontrollgruppe demonstriert wurden, wurden videografiert und in die Lernvideos für die Treatmentgruppe integriert. Im Unterricht der Kontrollgruppe blieb mehr Zeit zum Lernen am Experiment, weil es organischer Bestandteil des fragend-entwickelnden Physikunterrichts ist. Aufgrund der Kürze der Lernvideos für die Treatmentgruppe sahen die Schüler zwar dieselben Experimente, doch in wesentlich kompakterer Form.

An die Unterrichtsreihe knüpfte sich gemäß Lehrplan die Erzeugung und Ausbreitung elektromagnetischer Wechselfelder und Wellen an, sie war aber nicht mehr inhaltlicher Teil der Studie. 


\subsection{Pool an Übungsaufgaben}

Für die Kontroll- und Treatmentgruppe wurde von den an der Studie teilnehmenden Lehrkräften vorab ein Pool von etwa 16o Aufgaben zusammengetragen, geprüft, geordnet und mit Musterlösungen versehen. Dieser Aufgabenpool wurde fixiert und im Laufe der Studie nicht mehr verändert. Die Aufgaben entstammten verschiedenen Quellen, nämlich den in den Physikkursen verwendeten Lehrwerken (Grehn \& Krause 2009; Herrmann-Rottmair et al. 2009), ehemaligen bayerischen Abituraufgaben, Aufgaben des Online-Unterrichtsmittelanbieters LEIFI (Martin 2018) sowie von den Lehrkräften selbst erstellten, ehemaligen Klausuraufgaben.

Für die Kontrollgruppe wurde der Aufgabenpool dafür genutzt, dass die Schüler neu Erlerntes im Unterricht einüben, anwenden und vertiefen konnten. Die Hausaufgaben für die Kontrollgruppe wurden ebenfalls dem Pool entnommen. Dabei war für die Lehrkraft eine mittlere Hausaufgabendauer von 10-15 Minuten das Richtmaß bei der Auswahl der Aufgaben. Da das Fach Physik in allen Kursen pro Woche einmal doppel- und einmal einstündig unterrichtet wurde, mussten die Schüler der Kontrollgruppe dementsprechend zweimal pro Woche Hausaufgaben anfertigen. Für die Treatmentgruppe wurde der Aufgabenpool ausschließlich genutzt, um die Inhalte der Lernvideos im Unterricht zu festigen und zu vertiefen. Die Verwendung des Aufgabenmaterials erfolgte für die Treatmentgruppe also vollständig in der Schule.

Die Festlegung eines gemeinsamen Aufgabenpools diente dazu, einheitliches Übungsmaterial in beiden Gruppen verwenden zu können und so eine qualitative Vergleichbarkeit herzustellen. Es wurde jedoch darauf verzichtet, feste Aufgaben für die Unterrichtsstunden zu definieren. Das sollte im Ermessen der Lehrkraft bleiben, um der Unterrichtsdynamik und dem pädagogisch-didaktischen Handeln der einzelnen Lehrkraft ausreichend Raum zu lassen.

\subsection{Lernvideos und Clicker-Quiz}

Für die Treatmentgruppe wurde der gesamte Inhalt der Unterrichtsreihe auf 16 instruktive Lernvideos aufgeteilt. Die Lehrkräfte der Treatmentgruppe übernahmen die Produktion von je zwei bis sechs zusammenhängenden Videos. Dabei sprachen sie sich eng ab, berieten sich gegenseitig, erstellten die ihnen zugeteilten Videos aber in eigener Verantwortung.

Inhaltsübersicht über die Videos: Nachfolgend gibt Tab. 2 Aufschluss über die Sequenzierung der Unterrichtsreihe in die Lernvideos für die Treatmentgruppe. Die Videoproduktion verlief arbeitsteilig. Für die Schüler der Treatmentgruppe bedeutete das, dass sie zwar auch Videos des eigenen Lehrers, aber überwiegend Videos von Physiklehrern anderer Kurse beziehungsweise einer anderen Schule sahen. Somit ist die vorgegebene Instruktionssituation für alle Schüler der Treatmentgruppe ähnlich. 
Tab. 2. Themen, Dauer und Verantwortung für die Lernvideos für die Treatmentgruppe

\begin{tabular}{|c|c|c|c|c|}
\hline Nummer & Inhalt & & Dauer & Verantwortlich \\
\hline 1 & \multirow{8}{*}{ Induktion } & Grundlagen der Induktion & $8: 25 \min$ & Lehrer 1 \\
\hline 2 & & Induktion durch Flächenänderung & $7: 28 \min$ & Lehrer 1 \\
\hline 3 & & Induktion durch Magnetfeldänderung & $8: 40 \mathrm{~min}$ & Lehrer 1 \\
\hline 4 & & Lenzsche Regel & $7: 52 \min$ & Lehrer 1 \\
\hline 5 & & Wirksame Fläche & 4:51 min & Lehrer 1 \\
\hline 6 & & $\begin{array}{l}\text { Anwendung: Transformator und Induktions- } \\
\text { herd }\end{array}$ & $7: 23 \min$ & Lehrer 1 \\
\hline 7 & & Erzeugung von Wechselspannung & 8:11 $\min$ & Lehrer $5^{7}$ \\
\hline 8 & & Effektivwerte der Wechselspannung & $7: 23 \min$ & Lehrer 5 \\
\hline 9 & \multirow{4}{*}{ Selbstinduktion } & Grundlagen der Selbstinduktion & $5: 33 \min$ & Lehrer 2 \\
\hline 10 & & Ein- und Ausschaltvorgang bei einer Spule & $7: 53 \mathrm{~min}$ & Lehrer 2 \\
\hline 11 & & Magnetische Energie einer Spule & $8: 00 \mathrm{~min}$ & Lehrer 2 \\
\hline 12 & & Anwendung: Zündung von Leuchtstofflampen & $4: 32 \min$ & Lehrer 2 \\
\hline 13 & \multirow{4}{*}{ Schwingkreis } & $\begin{array}{l}\text { Einführung zum elektromagnetischen } \\
\text { Schwingkreis }\end{array}$ & $5: 39 \min$ & Lehrer 3 \\
\hline 14 & & Ungedämpfte Schwingung und Energien & $6: 19 \min$ & Lehrer 3 \\
\hline 15 & & Thomsonformel & $5: 00 \mathrm{~min}$ & Lehrer 3 \\
\hline 16 & & Erzwungene Schwingung und Resonanz & $9: 44 \min$ & Lehrer 3 \\
\hline
\end{tabular}

Gestaltung der Videos: Die formalen Vorgaben für die Lernvideos, an die sich die Lehrkräfte bei der Produktion gehalten haben, entstammen den Praxisempfehlungen zum Flipped Classroom (Flipped Learning Network 2014; Leibniz-Institut für Wissenschaftsmedien 2017; Bergmann \& Sams 2012; Douglas et al. 2017; Kück 2014) und der gemeinsamen Festlegung. Jedes Video wird mit einer Titelfolie und einem Jingle eingeleitet und besitzt eine dreiteilige Struktur:

(a) Einleitung: Den Schülern wird anhand einer Folie kurz vorgestellt, worum es inhaltlich geht und was sie durch dieses Video lernen werden.

7 Wie in Kap. 5.1.2 zu sehen sein wird, gab es einen Lehrerwechsel. Lehrer 4 hat nur einen Kurs der Kontrollgruppe unterrichtet, Lehrer 5 hingegen nur einen Kurs der Treatmentgruppe. 
(b) Hauptteil: Mit Hilfe realer, videografierter Demonstrationsversuche und kommentierter Folienpräsentationen werden die neuen Inhalte dargeboten. Die Versuche wurden in den Physikfachräumen der beteiligten Schulen aufgenommen. Im Video erläutert die Lehrkraft den Versuch, während sie ihn durchführt und betont die zentralen Inhalte, die an ihm deutlich werden. Ergänzt wird die Versuchsaufzeichnung durch Voice-over-screencasts von Folienpräsentationen, in denen weitere Erklärungen gegeben und Formalismen dargestellt werden. Dabei zeichnet eine Software den Inhalt eines Bildschirms auf, auf dem eine gewöhnliche Folien-präsentation ausgeführt wird. Zusätzlich wird über ein Mikrofon ein Audiokommentar aufgezeichnet, so dass das Video einer kommentierten Folienpräsentation entsteht. Mit einer Videobearbeitungssoftware werden die einzelnen Elemente (videografierte Demonstrationsversuche und Voice-over-screencasts) miteinander verbunden. Für die Screencasts und das Editieren der Videos wurden Camtasia Studio ${ }^{\circledR}$ 9.0 von TechSmith ${ }^{\mathrm{TM}}$ (Windows) beziehungsweise Kazam Screencaster 1.4 und kdenlive 16.0, GNU GPL v3, (Linux) verwendet.

(c) Sicherungsfolie: Am Ende des Videos präsentiert die letzte Folie kompakt alle Inhalte. Diese letzte Videoeinstellung ist von den Schülern ins Heft zu übernehmen. Die Sicherungsfolie garantiert, dass alle Schüler die wesentlichen Informationen in ihren Unterlagen haben und sie im Unterricht oder in der Vorbereitung auf eine Leistungserhebung darauf zurückgreifen können.

Das Richtmaß für die Länge der Videos lag bei 5-8 Minuten. Das ist nach Guo et al. (2014) ein sinnvoller Wert, um die Abbruchquote bei der Wiedergabe von Lernvideos niedrig zu halten. Den Folienpräsentationen lag eine einheitliche Masterfolie zugrunde, um den Videos trotz unterschiedlicher Urheber ein einheitliches Layout zu geben. Aus diesem Grund trugen auch die Lehrkräfte in den Videos einheitlich schwarze Hemden. Die nachfolgenden Abbildungen sollen die Art der Videos veranschaulichen.

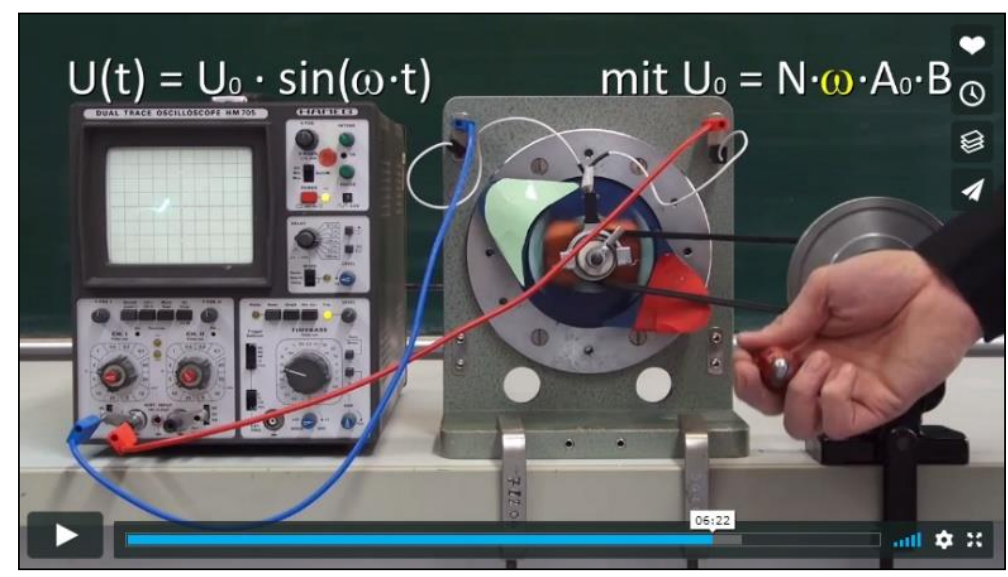

Abb. 6. Beispiel: Versuch aus dem Lernvideo Nr. 8 


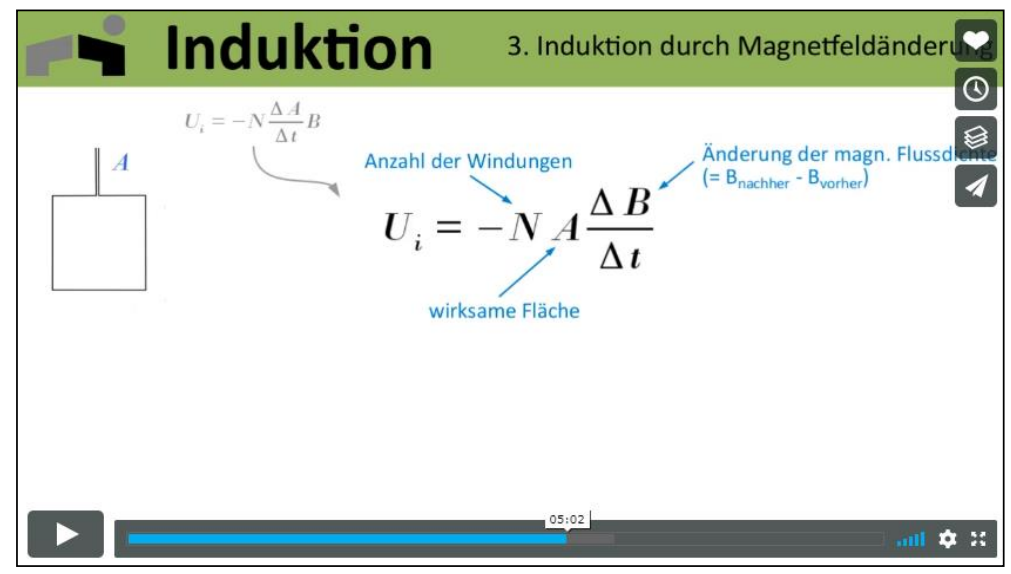

Abb. 7. Beispiel: Herleitung aus dem Lernvideo Nr. 3

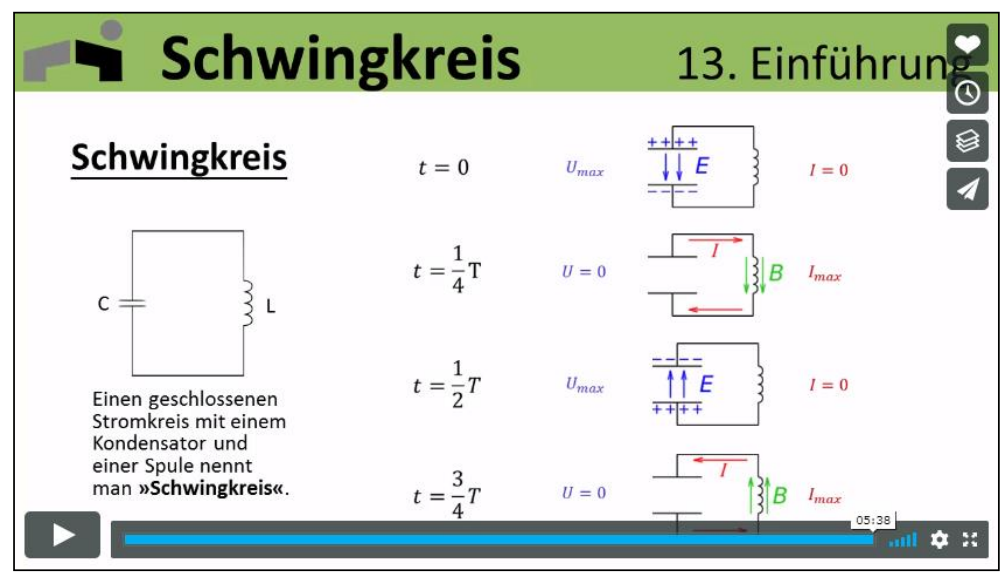

Abb. 8. Beispiel: Sicherungsfolie aus dem Lernvideo Nr. 13

Lernplattform: Die Lernvideos wurden sukzessive auf dem Videoportal Vimeo ${ }^{\mathrm{TM}}$ für die Schüler online gestellt. ${ }^{8}$ Zusätzlich wurde ein virtueller Kursraum auf der bayerischen Lernplattform MEBIS (BStMUK, Bayerisches Staatsministerium für Unterricht und Kultus 2018) verwendet. Hier mussten die Schüler sich anmelden, um die Videos ansehen zu können. Ergänzend zu Vimeo konnten die Schüler bei MEBIS auf die Aufgabenblätter der vergangenen Unterrichtsstunden und ihre Lösungen zugreifen. Dieses Angebot war optional. Es wurde gewährleistet, dass alle Schüler einen aktiven Zugang zu MEBIS hatten.

Clicker-Quiz: Das Clicker-Quiz ist die Verbindung der Lernvideos zum Präsenzunterricht (vgl. Kap. 2.3.2). Zu jedem Lernvideo wurden vier PowerPoint ${ }^{\circledR}$-Präsentationsfolien mit Single- oder Multiple-Choice-Fragen erstellt, die über ein Add-on der CPS-Abstimmungssoft${\text { ware } \text { FLOW }^{\circledR} \text { 2.0 von eInstruction }}^{\mathrm{TM}}$ zu interaktiven Clicker-Fragen wurden. Zu Beginn der

8 https://vimeo.com/album/4388279/.

Mittlerweile sind die Videos unter Creative Commons-Lizenz auf dem Videoportal YouTube ${ }^{\mathrm{TM}}$ verfügbar: https://www.youtube.com/channel/UCDR8jjd85JxrENhbz97FN5Q/playlists. 


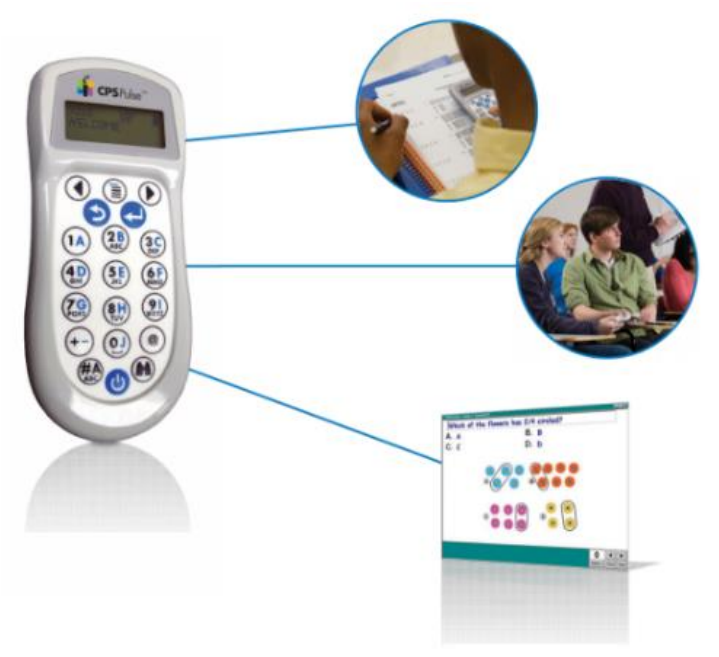

Abb. 9. Abfragegerät CPS-Pulse ${ }^{T M}$ für das Clicker-Quiz (aus Adidact 2018)

Unterrichtsstunde bekam jeder Schüler einen Clicker CPS-Pulse ${ }^{\mathrm{TM}}$ (vgl. Abb. 9), mit Hilfe dessen er an dem Quiz teilnehmen konnte.

Die ersten Fragen eines jeden Quiz wurden so gestaltet, dass sie zu beantworten sind, wenn das Lernvideo gesehen wurde. Sie besaßen rein reproduktiven Charakter. Danach zielten die Fragen auf das konzeptuelle Verständnis des Inhalts ab und erforderten kognitive Reorganisation oder Transfer. Untenstehend zeigt Abb. 10 eine Quizfrage, die über die Buchstabentasten der Clicker beantwortet wird.

Jedes Quiz bestand aus genau vier Fragen und wurde von der Lehrkraft erstellt, in deren Verantwortung auch das Lernvideo stand. Diese Lehrkraft schlug auch aus dem Aufgabenpool eine Reihe von Aufgaben vor, die sich im Anschluss am besten dafür eigneten, im Unterricht bearbeitet zu werden.

1. Induktion
A | Der Teil der Fläche einer Spule, der sich im Magnetfeld befindet
B | Die vertikale Projektionsfläche einer Spule im Magnetfeld
C | Der Anteil der Fläche einer Spule, der senkrecht vom Magnetfeld
durchsetzt ist
D | Die Projektion der Spulenfläche auf die Ebene, die senkrecht zu den
Magnetfeldlinien steht

Abb. 10. Beispiel: Folie aus dem Clicker-Quiz zu Lernvideo Nr. 5 


\section{Untersuchungsmethode und Instrumente}

Nachdem in Kap. 4 die Materialien und Medien vorgestellt wurden, die in dieser Studie eingesetzt wurden, soll zunächst das Design erläutert werden, an dem die Untersuchung ausgerichtet war. Daran schließen sich die Dokumentation des Unterrichts in der Kontroll- und der Treatmentgruppe sowie die Beschreibung der Messinstrumente an.

\subsection{Untersuchungsdesign}

Um die Forschungsfragen zu beantworten, wurde ein quasiexperimentelles Pre/Post-Design mit Kontrollgruppe gewählt. Quasiexperimentell ist die Studie, da die Schüler im Lernfeld Schule nicht randomisiert in Gruppen eingeteilt werden können, sondern in ihrer bestehenden Kurszusammensetzung bleiben und von ihren eigenen Fachlehrern unterrichtet werden. Das ist vor allem in der Oberstufe wichtig, um die Lernkontinuität hinsichtlich der Abiturvorbereitung zu gewährleisten. Eine Durchmischung der Schülerschaft zu Studienzwecken in einer abiturrelevanten Lernphase hätte großen Widerstand erzeugt.

Das Design der Wahl ist ein Cross-over-Design, bei dem zwei Unterrichtsreihen mit verschiedenen Methoden überkreuz unterrichtet werden, wie Tab. 3 veranschaulicht. Der große Vorteil des Cross-over-Designs ist, dass Aussagen über die Wirkung des Treatments stärker unabhängig von den teilnehmenden Schülern sind. Denn alle an der Studie teilnehmenden Schüler erfahren traditionellen Unterricht und Flipped Classroom, wobei gegen die jeweils andere Gruppe getestet wird.

Die Gründe, warum jedoch kein Cross-over-Design für diese Studie verwendet wurde, waren praktische. Die an der Studie teilnehmenden Lehrkräfte erklärten sich zu einem ohnehin schon großen unterrichtlichen Mehraufwand bereit. Die engen Absprachen für den Unterricht sowohl in der Kontroll- als auch der Treatmentgruppe, die Fortbildung im Flipped Classroom, die Erstellung der Videos und der interaktiven Quizze und der Austausch über die verwendeten Übungsaufgaben nahmen viele Ressourcen in Anspruch. Eine Verdoppelung des Aufwands durch die Ausweitung der Methode auf eine weitere Unterrichtsreihe hätte Widerstände bei den Lehrkräften ausgelöst und wäre kontraproduktiv für die Durchführung der Studie gewesen. Eine Alternative wäre gewesen, das Treatment von acht auf vier Wochen zu kürzen und so in kleinerem Maßstab ein Cross-over-Design zu realisieren. Doch wurde die-

Tab. 3. Mögliches Design: Cross-over

\begin{tabular}{|c|c|c|}
\hline & Unterrichtsreihe 1 & Unterrichtsreihe 2 \\
\hline Gruppe A & traditionell & Flipped Classroom \\
\hline Gruppe B & Flipped Classroom & traditionell \\
\hline
\end{tabular}


se Überlegung verworfen, da der motivationale Neuheitseffekt bei Treatments mit neuen Medien von nur vier Wochen deutliche Effekte gezeigt hätte. Clark (1983) verweist auf die Dauer von mindestens acht Wochen, nach denen Neuheitseffekte nur noch schwach ausgeprägt seien.

Um die Rolle der Lehrkraft beim Einsatz der jeweiligen Unterrichtsmethode berücksichtigen zu können, sollten alle Lehrkräfte jeweils beide Arten unterrichten. Physiklehrkräfte haben in der Praxis nie zwei Oberstufenkurse in einer Jahrgangsstufe gleichzeitig. Deshalb war es notwendig, die Studie auf zwei Schuljahre auszudehnen, in denen die Kurse der Kontrollund Treatmentgruppe nacheinander unterrichtet wurden. So stellt sich das Design als asynchrones Pre-/Post-Design mit Kontrollgruppe dar, wie Tab. 4 illustriert.

Das gewählte Design entspricht dem, das auch in anderen Methodenvergleichsstudien zum Flipped Classroom verwendet wurde (z.B. McLaughlin et al. 2014; Şengel 2016). Zwei deutliche Einschränkungen dieses Designs sind zu nennen, die die interne Validität der Messverfahren einschränken: Mögliche zwischenzeitliche Geschehen sorgen für Varianz. Es ist nicht klar und zu erheben, welche Konstellationen und schulischen Ereignisse einen Einfluss auf die Schüler des Treatmentjahrgangs hatten, die es für den Kontrolljahrgang nicht gab. AuBerdem unterrichteten alle Lehrkräfte den Flipped Classroom als zweite Methode. Auch hier liegt eine Konfundierung vor.

Es besaß jedoch Organisationsvorteile, dass alle vier Lehrkräfte geschlossen zuerst die Kontrollgruppe und im Folgejahr die Treatmentgruppe unterrichtet haben. Parallel zum Unterricht in der Kontrollgruppe konnten gemeinsame Überlegungen für die Videos in der Treatmentgruppe angestellt werden. Die experimentellen Aufbauten aus dem Unterricht in der Kontrollgruppe konnten für die Videoaufzeichnungen für die Treatmentgruppe verwendet werden. Die gesamte unterrichtsorganisatorische Planung des Flipped Classroom lief synchron zum Unterricht in der Kontrollgruppe. Dadurch wurde auch bewirkt, dass der Austausch zum Unterricht in der Kontrollgruppe hoch und die inhaltliche Parallelität des Unterrichtsgeschehens groß war.

Der Konfundierungseffekt, der sich ergibt, weil alle Lehrkräfte zuerst traditionell und anschließend im Flipped Classroom unterrichteten, sorgte deshalb auf der anderen Seite dafür, dass die Unterrichtsabläufe in allen Kursen beider Schuljahre eine große Parallelität aufwiesen. Die Kontrolle über die Ähnlichkeit der Unterrichtsabläufe war deshalb größer, als wenn die Lehrkräfte parallel nach unterschiedlichen Methoden unterrichtet hätten.

Tab. 4. Verwendetes Studiendesign: Asynchrones Pre-/Post-Design mit Kontrollgruppe

\begin{tabular}{|c|c|c|}
\hline & Schuljahr 2015/16 & Schuljahr 2016/17 \\
\hline Kontrollgruppe & traditionell & \\
\hline Treatmentgruppe & & Flipped Classroom \\
\hline
\end{tabular}




\subsubsection{Schulorganisatorischer Rahmen}

Es wurden Schulleitungen und Physiklehrkräfte örtlicher Gymnasien angefragt, ob Möglichkeit und Bereitschaft vorhanden seien, sich an der Studie im Rahmen einer schulinternen Evaluation mit externer Beratung zu beteiligen. Eine schulinterne Evaluation ist die Bewertung von Prozessen oder Verfahren einer Schule durch Personen, die ihr selbst angehören, und besitzt eine hohe Bedeutung für die Schulentwicklung (ISB, Staatsinstitut für Schulqualität und Bildungsforschung 2010). Die Schulleitung besitzt rechtlich die Kompetenz, über eine schulinterne Evaluation zu entscheiden. Zwei Würzburger Gymnasien fanden sich, an denen eine solche Evaluation stattfinden konnte, Schule A und Schule B. An ihnen war üblicherweise mit insgesamt vier Physikkursen (drei an Schule A und einem an Schule B) in der 11. Jahrgangsstufe zu rechnen. Da der Autor dieser Studie sowohl den externen Berater darstellt als auch Lehrkraft der Schule A ist, ist die Verfahrensweise juristisch abgesichert. Dass der Autor gleichzeitig auch Teilnehmer der Studie ist, beeinträchtigt die Objektivität der Messverfahren und ihrer Auswertung. Diese Störung wurde in Kauf genommen, um trotz der lokalen Beschränkung auf zwei Gymnasien die Stichprobe groß zu halten.

Im derzeit noch gültigen Lehrplan des achtjährigen Gymnasiums in Bayern zählt die 11. Jahrgangsstufe zur Qualifikationsphase des Abiturs. Mit der Einführung des achtjährigen Abiturs fiel die Differenzierung von Leistungs- und Grundkursen weg. Das bedeutet, dass Schüler in Bayern keine Möglichkeit haben, Leistungs- oder Grundkurse, sondern nur noch Einheitskurse zu wählen. Die Physikkurse in der 11. Jahrgangsstufe besitzen deshalb in der Regel eine größere Leistungsheterogenität als Grund- oder Leistungskurse in anderen Bundesländern. Die naturwissenschaftlichen Fächer Physik, Chemie und Biologie sind jeweils dreistündig und decken etwa den Lehrplaninhalt der früheren Grundkurse ab. Mindestens ein naturwissenschaftliches Fach muss bis zum Abitur belegt werden; häufig haben Schüler in der 11. Jahrgangsstufe noch zwei naturwissenschaftliche Fächer, von denen sie eins in die 12. Jahrgangsstufe weiterführen.

\subsubsection{Teilnehmende Lehrkräfte}

Wie oben erwähnt besaßen die an der Studie teilnehmenden Physiklehrkräfte mindestens fünf Jahre Berufserfahrung und haben die 11. Jahrgangsstufe zuvor mindestens drei Mal unterrichtet. Damit war eine gewisse Souveränität und Vertrautheit mit den Experimenten, den Lehrplananforderungen und den Leistungserwartungen hinsichtlich des Abiturs gewährleistet. Aufgrund des organisatorischen Rahmens einer schulinternen Evaluation war die Zahl der teilnehmenden Lehrkräfte niedrig. An den beiden Gymnasien konnten drei Lehrkräfte (Schule A) und eine Lehrkraft (Schule B) gefunden werden. Diese vier Lehrkräfte sollten insgesamt acht Physikkurse in zwei hintereinander folgenden Schuljahren - 2015/16 und 2016/17 - unterrichten. Leider zeigte sich im Verlauf, dass die schulische Personalplanung am Gymnasium B den Einsatz ihrer Lehrkraft im zweiten Jahr der Studie nicht mehr gewährleisten 
konnten, so dass es dort einen personellen Wechsel gab. Nur die drei Lehrkräfte des Gymnasiums A unterrichteten demnach beide Unterrichtsformen - traditionell und Flipped Classroom.

Alle unterrichtenden Lehrkräfte waren männlich. Da eine der Forschungsfragen auf den Geschlechtereffekt beim Flipped Classroom abzielt, erhöht es die interne Validität, wenn das Geschlecht der Lehrkräfte nicht variiert, selbst wenn der Effekt des Lehrergeschlechts sonst keine Berücksichtigung findet.

Die Lehrkräfte wurden für die Studie durch die Inhalte spezieller Fortbildungen in der Unterrichtsmethode Flipped Classroom durch die Akademie für Lehrerfortbildung und Personalführung, Dillingen a.d. Donau, vom 26.10. bis 23.11.2015 (Moderiertes Online-Seminar) und 21. bis 23.3.2016 (Fachtagung in Dillingen) geschult.

\subsubsection{Dauer und Ablauf der Untersuchung}

In der nachfolgenden Übersicht ist der zeitliche Verlaufsplan der Studie dargestellt. Innerhalb der achtwöchigen Unterrichtsreihe gab es sowohl für die Kontroll- wie für die Treatmentgruppe zwei Unterbrechungen durch Ferienblöcke, so dass die Unterrichtsreihe für alle Schüler in drei Blöcke aufgeteilt war, die sich über drei Monate erstreckten. Nach Clark (1983) ist davon auszugehen, dass motivationale Neuheitseffekte durch technologiegestützte Interventionen nach einem Zeitraum von acht Wochen nur noch schwach ausgeprägt seien. Durch die Ferienunterbrechungen dehnte sich der Flipped Classroom für die Treatmentgruppe auf drei Monate aus, so dass dem Neuheitseffekt keine größere Bedeutung zuzumessen ist.

Geplant war, dass innerhalb der Unterrichtsreihe in keinem der Kurse eine schriftliche Leistungserhebung im Physikunterricht stattfinden sollte. Etwa ein bis zwei Schulwochen nach dem Posttest war in beiden Gruppen die reguläre Physikklausur angesetzt, von denen eine pro Halbjahr geschrieben wird. Da der Posttest in zeitlicher Nähe zur Physikklausur stand, wurde den Schülern nahegelegt, sich - trotz Pseudonymisierung - im Fachwissensteil besonders anzustrengen, um selber einen Eindruck zu bekommen, wo sie noch Defizite im Hinblick auf die Klausur besäßen. Eine Ausnahme bildete die Schule B. Dort wurde aus organisatorischen Gründen die reguläre Physikklausur für den Kurs der Kontrollgruppe mitten in die Unterrichtsreihe gelegt. Eine Musterlösung zum Fachwissenstest wurde im Anschluss an die Unterrichtsreihe nicht ausgegeben. Nachfolgend zeigt Tab. 5 einen zeitlichen Überblick über den Studienverlauf. Ein detaillierter Zeitplan beider Gruppen findet sich im Anhang (s. Anhang 1).

Eine Follow-up-Test nach vielleicht vier, sechs oder acht Wochen hätte Aussagen über die Nachhaltigkeit des Lernens im Flipped Classroom erlaubt. Da jedoch in allen Kursen zu unterschiedlichen Zeitpunkten nach Ende der Studie Klausuren geschrieben wurden, hätte die individuelle Lernvorbereitung der Schüler zu Verzerrungen geführt. Zudem erschien eine zusätzliche Inanspruchnahme von Unterrichtszeit zu Testzwecken den meisten Lehrkräften unangemessen. Aus diesen Gründen wurde auf einen Follow-up-Test verzichtet. 
Tab. 5. Zeitlicher Verlaufsplan der Studie

\begin{tabular}{|c|c|c|}
\hline März 2016 & Pretest & \multirow{3}{*}{$\begin{array}{c}\text { Kontrollgruppe } \\
\text { (= } 4 \text { Kurse) }\end{array}$} \\
\hline März - Juni 2016 & $\begin{array}{l}3 \text { Monate: traditionell } \\
1 \text { Woche Unterricht, dann } 2 \text { Woche Ferien } \\
6 \text { Wochen Unterricht, dann } 2 \text { Wochen Ferien } \\
1 \text { Woche Unterricht }\end{array}$ & \\
\hline Juni 2016 & Posttest & \\
\hline Februar 2017 & Pretest & \multirow{3}{*}{$\begin{array}{l}\text { Treatmentgruppe } \\
\qquad \text { (= } 4 \text { Kurse) }\end{array}$} \\
\hline Februar - Mai 2017 & $\begin{array}{l}3 \text { Monate: Flipped Classroom } \\
2 \text { Wochen Unterricht, dann } 1 \text { Woche Ferien } \\
5 \text { Wochen Unterricht, } 2 \text { Wochen Ferien } \\
1 \text { Woche Unterricht }\end{array}$ & \\
\hline Mai 2017 & Posttest & \\
\hline
\end{tabular}

\subsection{Beschreibung des traditionellen Unterrichts}

Der traditionelle Physikunterricht, der in der Kontrollgruppe gehalten wurde, richtete sich an dem aus, was die teilnehmenden Lehrkräfte als etablierte Praxis in ihrem eigenen Unterricht identifizierten: ein fragend-entwickelnder und am Experiment orientierter chalk \& talk-Unterricht, in dem immer wieder Gelegenheiten geschaffen werden, dass die Schüler selbstständig Aufgaben zur Anwendung und Vertiefung bearbeiten - während des Unterrichts und zu Hause. Der Physikunterricht, der in dieser Studie als »traditionell« bezeichnet wird, ist keineswegs das Zerrbild einer vorlesungsähnlichen Lehrerinstruktion nach dem Modell des Nürnberger Trichters. Auch der traditionelle Physikunterricht steht unter dem Anspruch, schüleraktivierend zu sein, selbst wenn er zum großen Teil aus Frontalunterricht besteht.

Folgende Strukturelemente charakterisierten den Physikunterricht, auf die sich die beteiligten Lehrkräfte vorab im gemeinsamen Austausch festgelegt hatten und die in der Kontrollgruppe Anwendung fanden:

Rückbindung an die letzte Stunde/ Besprechung der Hausaufgaben: In der ersten Phase des Unterrichts werden die zentralen Unterrichtsinhalte der letzten Unterrichtsstunde in Form eines Lehrer-Schüler-Gesprächs rekapituliert. Außerdem werden die schriftlichen Hausaufgaben besprochen, die in der Vorstunde zur Bearbeitung aufgetragen wurden. Bei einfachen Aufgaben werden lediglich mündlich die Ergebnisse verglichen, bei komplexeren Aufgaben nimmt die Lehrkraft eine verkürzte Verschriftlichung an der Tafel unter Zuhilfenahme von Schülerbeiträgen vor. Alternativ kann auch ein Schüler seine Lösung an die Tafel bringen, doch geschah das in der Praxis eher selten, weil es in der Regel wesentlich mehr Zeit in Anspruch nimmt. 
Im traditionellen Unterricht der Oberstufe gibt es keine Hausaufgabenkontrolle, so dass die Schüler sich in der Regel auch nicht meldeten, wenn sie keine Hausaufgaben angefertigt hatten.

Motivation und Erarbeitung neuen Inhalts unterstützt durch Experimente: Den quantitativ größten Teil nimmt die Hinführung zu und Erarbeitung von neuen Inhalten ein. Ausgangspunkt sind dabei stets vom Lehrer durchgeführte und gegebenenfalls von Schülern assistierte Demonstrationsversuche. Dies können einfache Handversuche mit wenigen Hilfsmitteln sein (Stabmagnet, Spule und Voltmeter für Induktionsspannung; Kupferrohr und Neodymmagnet für Lenzsche Regel) oder komplexe Versuche, die entweder vorab auf einem Experimentierwagen vorbereitet wurden oder Schritt für Schritt im Unterricht mit erklärenden Hinweisen aufgebaut werden (Ausschaltvorgang in einer Spule, elektromagnetischer Schwingkreis). Die Experimente können qualitativen oder quantitativen Charakter besitzen. Begleitend zu den Experimenten regen Lehrerinformationen, -fragen und -impulse die Schüler zum aktiven Mitdenken an. Vor, zwischen und nach den Experimenten sichert der Lehrer Ergebnisse an der Tafel, die von den Schülern zur Sicherung ins Heft übernommen werden. Falls ganze Messwertreihen erhoben werden, kann die Assistenz durch Schüler darin bestehen, dass einzelne Schüler vorkommen, Geräteeinstellungen vornehmen, Messwerte ablesen und Messwerte an der Tafel sichern.

Verbunden mit den Experimenten sind grafische oder numerische Auswertungen, Herleitungen, Bestätigungen und Beispielrechnungen. Diese tafelgestützten Lernschritte werden durch den Lehrer unter Einbeziehung der Schüler gesteuert. Das fragend-entwickelnde Lehrer-Schüler-Gespräch ist auch hier die dominierende Kommunikationsform, die immer wieder ergänzt wurde durch kurze Phasen der Einzelarbeit (etwa Umrechnen von Messwerten oder Folgeberechnungen) oder Partnerarbeit (etwa gemeinsames Nachdenken über eine Frage). Dabei ist der Dreischritt des think-pair-share (Lyman 1981) ein gängiges Vorgehen zur Schüleraktivierung.

Vertiefen und Festigen des Wissens durch problemlösende Aufgaben: Nachdem in der Sicherungsphase neues Wissen schriftlich fixiert wurde, schließt sich die Phase der Vertiefung an. Sie bildet üblicherweise die letzte Phase der Unterrichtsstunde oder -doppelstunde. Sie kann aber auch - nach elementaren Lernschritten - zu früheren Zeitpunkten genutzt werden, woran sich dann weitere Herleitungen anschließen. Je zentraler ein neuer Lerninhalt innerhalb der Unterrichtsreihe ist, desto mehr Unterrichtszeit muss den Schülern für die Vertiefung und Festigung des Wissens eingeräumt werden. So kann es durchaus sein, dass eine komplette Einzel- oder Doppelstunde aufgewendet wird, um intensiv Lernaufgaben, etwa ehemalige Abituraufgaben, zu den neuen Sachverhalten zu bearbeiten. Die Aufgaben, die während der Unterrichtszeit bearbeitet werden, werden nach Möglichkeit noch in dieser besprochen - je nach Schwierigkeitsgrad entweder mündlich oder schriftlich. Gegebenenfalls wird die Besprechung an den Anfang der nächsten Stunde verlegt.

Die Hausaufgaben bilden die Verlängerung der Vertiefungsphase von der Schule nach Hause. Von Schülern der Oberstufe ist durchaus ein höheres zeitliches Maß zu erwarten, das sie für die Hausaufgaben aufwenden, als dies noch in der Mittelstufe üblich ist. Doch zeigt die 
praktische Erfahrung der Lehrkräfte im Überblick über die angefertigten Hausaufgaben, dass die Mehrheit der Schüler selten mehr als 15 Minuten Zeit investiert, um diese zu erledigen. Deshalb gilt eine Bearbeitungsdauer von 15 Minuten als Richtwert für die Menge zu erledigender Textbuch- oder Arbeitsblattaufgaben. Die Auswahl der Hausaufgaben liegt allein in der Verantwortung der unterrichtenden Lehrkraft. Aus dem Aufgabenpool (vgl. Kap. 4.2) wählt der Lehrer Aufgaben aus, die seiner Einschätzung nach am geeignetsten scheinen, die Lernprozesse der Schüler außerhalb des Unterrichts weiterzuführen.

Diese Charakteristika prägten den Physikunterricht in der Kontrollgruppe. Es wäre nicht zielführend gewesen, die Lehrkräfte vorab auf engere inhaltliche und methodische Richtlinien zu verpflichten. Denn erstens erfordert die Dynamik der Lernprozesse in jedem einzelnen Kurs eine gewisse Offenheit und Flexibilität von Seiten der Lehrkraft. Eine vollkommen uniforme Unterrichtsstruktur wäre zu starr gewesen, um den Fragen, Bedürfnissen und Schwierigkeiten der jeweiligen Lerngruppe gerecht zu werden. Zweitens muss die Lehrkraft Lerninstrumente, Arbeitsformen und Zeiträume spontan umgestalten können, um auf unvorhergesehene Änderungen reagieren zu können. Und drittens muss die Lehrkraft auch Raum für persönliche methodischen Präferenzen erhalten, da diese Teil eines authentischen Unterrichts für die Schüler sind. Deshalb garantieren die Strukturelemente zwar einerseits eine gewisse Einheitlichkeit des »traditionellen Physikunterrichts « in den vier Kursen der Kontrollgruppe, sind aber andererseits keine Blaupause für uniformen Unterricht.

\subsection{Beschreibung der Intervention}

Die Form des Flipped Classroom, der in der Treatmentgruppe eingesetzt wurde, deckt sich vollständig mit der in Kap. 2.3.2 definierten Basisform. Ihre Strukturelemente seien in Abb. 11 noch einmal grafisch dargestellt und auf die Lernsituation angepasst.

Instruction: Vorbereitend zu jeder Unterrichtsstunde sehen die Schüler ein einzelnes Lernvideo, unabhängig davon, ob es sich um eine Einzel- oder eine Doppelstunde handelt. Der Physikunterricht in der Oberstufe ist dreistündig, so dass in jeder Woche je eine Einzel- und eine Doppelstunde unterrichtet wird. Sukzessive werden dazu die durchnummerierten Lernvideos auf Vimeo ${ }^{\mathrm{TM}}$ und MEBIS freigegeben. Die Schüler sollen das jeweils aktuelle Video anschauen und werden angeregt, die Wiedergabe zu unterbrechen, wenn sie etwas nicht verstanden haben, zurückzuspringen und sich die entsprechende Stelle noch einmal anzusehen.

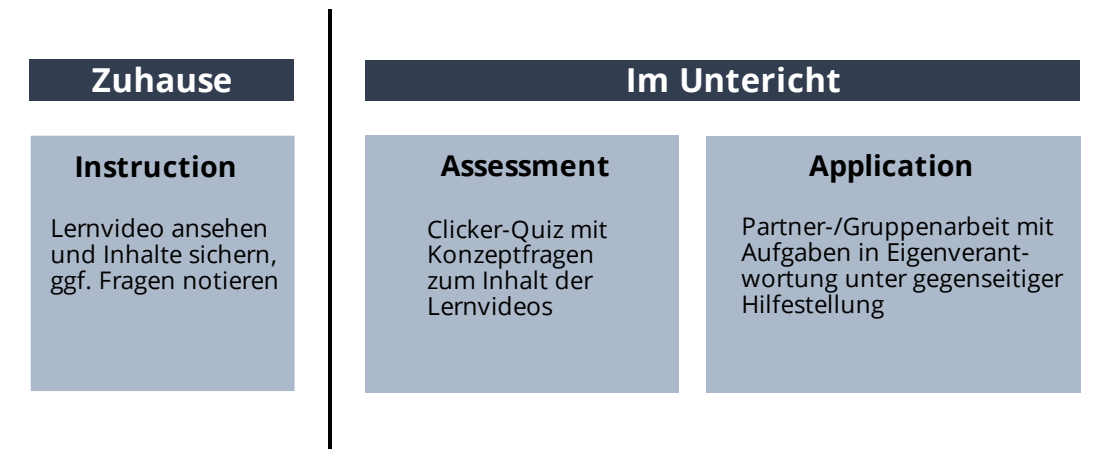

Abb. 11. Basisform des Flipped Classroom als Modell für die Studie 
Außerdem werden sie gebeten, die Fragen, die sich ihnen trotz mehrfachen Anschauens stellten, schriftlich festzuhalten, um sie später im Unterricht zu klären. Am Ende sollen sie die Sicherungsfolie, welche die letzte Einstellung des Lernvideos bildet, in ihr Heft übernehmen. Im Verlauf der Unterrichtsreihe wurde den Schülern jedoch gestattet, alternativ einen Screenshot der Sicherungsfolie auszudrucken.

Assessment: Jeder Schüler eines Kurses erhält zu Beginn der Stunde einen Clicker, mit dessen Hilfe er am Quiz teilnehmen kann, mit dem die Lehrkraft den Unterricht beginnt. Zunächst beantwortet jeder die Quizfrage, ohne mit seinen Nachbarn Rücksprache zu halten. Wenn die Fragezeit abgelaufen ist, die standardmäßig auf 1 Minute eingestellt ist, werden die Ergebnisse für alle sichtbar als Balkendiagramm angezeigt, ohne dass die richtige Lösung markiert wird. Haben über $75 \%$ der Schüler die richtige Lösung angegeben, wird die Frage aufgelöst und die Lehrkraft geht zur nächsten Frage über. Haben weniger als $75 \%$ der Schüler die richtige Lösung angegeben, erfolgt eine Peer Discussion von 1-2 Minuten, in der die Schüler mit ihren Nachbarn über die richtige Lösung diskutieren. Anschließend erfolgte eine erneute Lösungsabfrage und anschließend die Auflösung, zu der die Lehrkraft gegebenenfalls noch Erklärungen anfügt (vgl. Abb. 12). Jedes Quiz beinhaltet vier Fragen. Als Zeitvorgabe für das gesamte Quiz werden 10 Minuten angesetzt. Wenn nach dieser Zeit noch nicht alle Quizfragen bearbeitet sind, wird das Quiz entsprechend gekürzt. Hin und wieder war das in der Praxis der Fall. Es wurde deutlich, dass manche Quizfragen als Einstieg in den Unterricht zu schwer waren, um sie innerhalb von 10 Minuten zu bearbeiten.

Am Ende des Quiz haben die Schüler grundsätzlich noch Gelegenheit, spezifische Fragen zum Lernvideo zu stellen, welche von Mitschülern oder der Lehrkraft beantwortet werden. Dies war in der Praxis regelmäßig, aber nicht durchgängig, der Fall. Wesentliche Voraussetzung, dass die Schüler die Lernvideos schauen, ist, dass die Inhalte der Videos im Unterricht

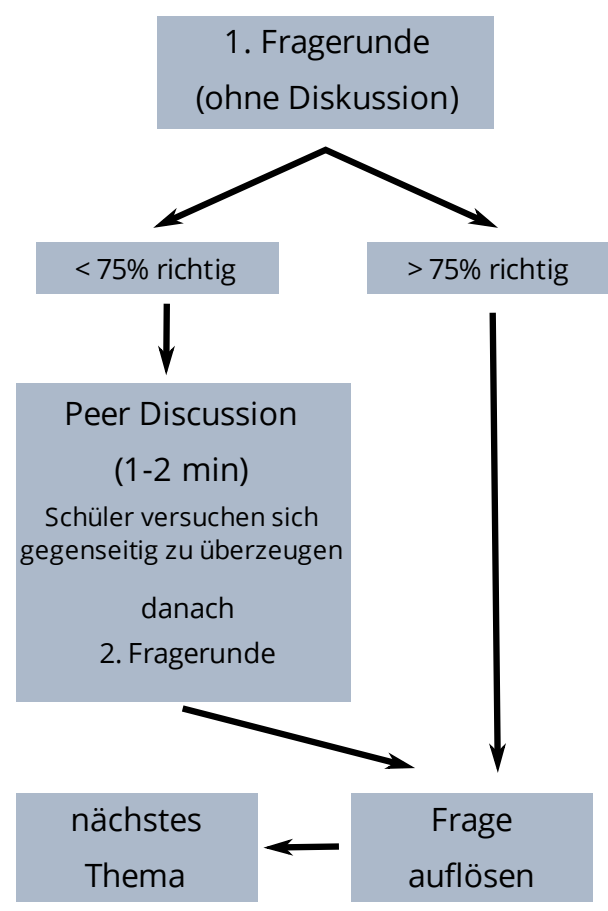

Abb. 12. Flussdiagramm für das Clicker-Quiz 
nicht noch einmal durch Mitschüler oder die Lehrkraft wiederholt oder zusammengefasst werden. Deshalb wurde vorab festgelegt, dass Bitten von Schülern, der ganze Inhalt solle nochmals erklärt werden, da sie »das ganze Video nicht verstanden « hätten, im Plenum nicht nachgegangen wird. Wenn sich dann im Einzelgespräch mit dem Schüler herausstellen sollte, dass das Video überhaupt nicht gesehen wurde oder kein Hefteintrag zum Video vorliegt, sollte ein pädagogisches Gespräch über die Verantwortung des Schülers für das eigene Lernen und die Relevanz der Videos für den eigenen Lernfortschritt erfolgen. Eine Zusammenfassung des Videos durch den Lehrer solle ein solcher Schüler jedoch nicht erhalten. Er sollte darauf angewiesen sein, aus den Aufgaben und im Gespräch mit seinen Mitschülern die wesentlichen Inhalte zu rezipieren. Dieser stark fordernde Anspruch an Schüler, welche die Videos nicht schauen, sollte alle Schüler darin unterstützen, sich konsequent mit den Lernvideos auseinander zu setzen. Tatsächlich trat dieser beschriebene Fall aber nur wenige Male am Anfang der Unterrichtsreihe auf.

Application: Den wichtigsten und umfangreichsten Teil des Unterrichts bildet die Phase, in der das neue Wissen angewendet wird. Die Schüler sollen hier Aufgaben bearbeiten, die in Passung zu den Inhalten der Videos aus dem Aufgabenpool (vgl. Kap. 4.2) ausgewählt werden. Die Lehrkraft, die das Video und die Quizfragen erstellt hat, macht vorab Vorschläge, welche Aufgaben aus dem Pool sich am besten im Anschluss an das Video eignen. Die letzte Verantwortung darüber, welche Aufgaben im Unterricht eingesetzt werden, liegt jedoch bei jedem Lehrer selbst.

In der Praxis zeigte sich allerdings, dass die Lehrkräfte den Vorschlägen ausnahmslos folgten und deshalb identische Arbeitsblätter für ihre Kurse verwendeten. Die Aufgaben jedes Arbeitsblatts besaßen unterschiedliche Anforderungsniveaus, von einfachen Formel- und Rechenaufgaben über beschreibende und analysierende Auswertungsaufgaben bis zu komplexen Transferaufgaben mit Abiturniveau. Neben Pflichtaufgaben, die alle Schüler zu bearbeiteten hatten, gab es häufig auch differenzierende Wahlaufgaben, zwischen denen die Schüler selbstständig oder nach Rat der Lehrkraft wählen konnten. Die Arbeitsblätter wurden samt den Lösungen in digitaler Form sukzessive im Kursraum der Lernplattform MEBIS online gestellt, was als zusätzliche Dienstleistung für die abwesenden Schüler gedacht war. Wie viele der Schüler davon Gebrauch gemacht haben, wurde in der Studie nicht erhoben.

Die Bearbeitung der Aufgaben sollte in kooperativer Form zu zweit oder zu dritt geschehen. Dabei können sich die Schüler entweder ihre Arbeitspartner selbst aussuchen oder mit ihren Sitznachbarn zusammenarbeiten. In einem der Kurse wurde in jeder Woche die Sitzordnung systematisch geändert, so dass es dort immer wechselnde Arbeitspartner gab. Bei Wahlaufgaben sollten sich die Mitglieder einer Arbeitsgruppe darauf einigen, welche Aufgaben sie bearbeiten wollen. Musterlösungen zu den Aufgaben liegen vorne am Lehrerpult aus, so dass die Schüler ihre Ergebnisse eigenständig kontrollieren und in ihrem eigenen Gruppentempo weiterarbeiten können. Manche Aufgaben mit besonders wichtigem Lerngehalt werden gemeinsam an der Tafel besprochen. Diese Vorgehensweise liegt im Ermessen der Lehrkraft und wurde regelmäßig praktiziert. 
In der Arbeitsphase der Schüler geht der Lehrer umher, verfolgt die Arbeit in den Gruppen und gibt Unterstützung, Hilfestellungen und Impulse. Das Betreuungsverhalten soll insgesamt defensiv ausgerichtet sein. Der Lehrer soll also kein Instructor im kleinen Kreis sein, sondern als Lernbegleiter versuchen, mit offenen Fragen sowie gestuften strategischen oder inhaltlichen Hilfen den individuellen Lernprozess der Schüler zu unterstützen. Entwickelt sich innerhalb einer Gruppe eine Diskussion über den richtigen Lösungsweg, soll die Lehrkraft zunächst nur mit Motivations- und Rückmeldungshilfen eingreifen, ohne inhaltliche Hinweise zu geben.

Am Ende der Unterrichtsstunde wird auf das jeweils neue freigegebene Lernvideo verwiesen. Die fortgesetzte Bearbeitung von ausstehenden Übungsaufgaben ist nicht vorgesehen. Gegebenenfalls erfolgt sie noch in der nachfolgenden Stunde.

\subsection{Messinstrumente}

Die vorliegende Studie fragt danach, ob der Flipped Classroom zu höheren kognitiven und affektiven Lernergebnissen im Unterrichtsfach Physik führt als traditionelle Lernformen. Eingeschränkt ist das Forschungsfeld auf das Gymnasium im deutschen Bildungssystem, das grundsätzlich unter dem Anspruch steht, Schüleraktivierung, Selbstbestimmung und Lernbegleitung angemessen zu realisieren. Um den Lernzuwachs in der Kontroll- und Treatmentgruppe vergleichen zu können, wurde ein Fachwissenstest konzipiert, der die Änderung der kognitiven Leistung testet, und ein Fragebogen, mit dem motivational-affektive Veränderungen sowie weitere Lernmerkmale erhoben werden.

Der Prezeitpunkt, zu dem der Fragebogen und der Fachwissenstest eingesetzt wurden, lag unmittelbar vor der Unterrichtsreihe, der Postzeitpunkt unmittelbar danach. In der Schule A fanden die Erhebungen in den drei Kontrollkursen und den drei Treatmentkursen zeitgleich in den jeweiligen Fachräumen statt. Die Erhebungen an der Schule B fanden jeweils mit einigen Tagen Versatz statt. Zum Zweck der streng parallelen Durchführung der Erhebung wurde eine Durchführungserläuterung mit festen Wortlautanweisungen erstellt (s. Anhang 2). Nach Bearbeitung des Fragebogens, für die etwa 15 Minuten veranschlagt wurden, erfolgte die Bearbeitung des Fachwissenstests, für die die Schüler maximal 7o Minuten Zeit hatten.

\subsubsection{Fachwissenstest}

Für die Unterrichtsreihe Elektromagnetische Induktion und elektromagnetischer Schwingkreis existiert kein standardisierter Fachwissenstest. Deshalb musste ein Testinstrument entwickelt werden, das vor und nach der Unterrichtsreihe eingesetzt werden konnte, um den Lernzuwachs der Schüler zu messen. Etablierte Wissenstests besitzen stets ein geschlossenes Format, sind also Multiple-Choice-Tests für Wissens- und Konzeptfragen. Der Vorteil geschlossener Tests liegt in der Eindeutigkeit, Objektivität und Ökonomie der Auswertemethoden. So ließe 
sich ein Fachwissenstest konstruieren, der den Inhalt der Unterrichtsreihe in Form von Multiple-Choice-Fragen abzubilden versucht. Das Problem eines Tests im geschlossenen Format liegt jedoch darin, dass viele Fertigkeiten, die im Physikunterricht der Oberstufe erlernt und eingeübt werden, nicht im Multiple-Choice-Format getestet werden können. Gerade der mathematische und kombinatorische Umgang mit Formeln, der in der Oberstufe eine wichtige Rolle einnimmt, lässt sich nicht adäquat im geschlossenen Format testen. Deshalb wurde die Entscheidung getroffen, einen offenen Fachwissenstest zu erstellen. Er sollte die Schüler in der Qualität fordern, wie es typischerweise durch Physikbuchaufgaben, Tests oder Physikklausuren geschieht.

Aufgaben aus etablierten Quellen wurden zusammengestellt, die in ihrer Summe das Format einer Oberstufenklausur im Fach Physik besaßen. Der Fachwissenstest bestand aus sechs Aufgaben, 4o Punkten und wurde für 70 Minuten Bearbeitungszeit konzipiert (s. Anhang 3). Der Test wurde von einem Expertenkreis aus fünf erfahrenen Physiklehrern des Gymnasiums A und vier Physikdidaktikern der Universität Würzburg geprüft und modifiziert. Weil sämtliche Aufgaben aus in Bayern zugelassenen Lehrbüchern und dem Archiv der bayerischen Physikabiturklausuren entnommen wurden und das abschließende Urteil der Experten einheitlich positiv war, wurde der Test direkt als Fachwissenstest in der Studie eingesetzt, ohne vorher mit anderen Schülern getestet worden zu sein.

Die ersten beiden Aufgaben A1 und A2 des Fachwissenstests bilden eine Ausnahme vom offenen Format und sind geschlossene Multiple-Choice-Aufgaben. Die Aufgaben A3 bis A6 sind unterteilt in insgesamt elf Unteraufgaben. In fünf von ihnen müssen die Schüler physikalische Größen berechnen, indem sie die richtigen Formeln miteinander kombinieren, umstellen und die Werte aus den Angaben einsetzen. Drei dieser fünf Aufgaben (A3b und c sowie A6b) sind echte Transferaufgaben, die nicht durch Aufgaben aus dem Aufgabenpool vorbereitet wurden. Sie machen elf von 40 Punkten aus (27,5\%). Die restlichen Aufgaben besitzen Reproduktions- (A1, A3a, A4a, A4b, A5a, A6a, A6c: 22 BE ^ 55\%) oder Reorganisationsniveau (A2, A4b, A6d: $7 \mathrm{BE} \hat{=}$ 17,5\%). Der im Vergleich zu einer regulären Klausur höhere Anteil an Reproduktionsaufgaben liegt darin begründet, dass der Fachwissenstest einen im Vergleich höheren Anteil an Konzeptaufgaben enthält, bei denen die Schüler erklären, erläutern und begründen müssen. Insgesamt entfallen 15 Punkte (37,5\%) auf solche Konzeptaufgaben. Mit ihnen sollten die Schüler zeigen, inwiefern sie die Konzepte der Induktion, Selbstinduktion und des Schwingkreises verstanden haben und artikulieren können.

Zur Auswertung des Fachwissenstests wurde eine kleinschrittige und transparente Musterlösung erstellt. Die Auswertung der Tests erfolgte durch den Autor. Eine zufällige Stichprobe von 30 der insgesamt 299 Fachwissenstests (10\%) der Pre- und Post-Erhebung wurde von einer weiteren beteiligten Lehrkraft zweitkorrigiert. Nur bei zwei Tests ergab sich eine Differenz von 0,5 Punkten, die übrigen deckten sich vollständig mit der Erstbewertung.

Da der Fachwissenstest eine Zusammenstellung von erprobten Aufgaben ist, die viele Gütekontrollen durchlaufen haben, ist von einer ausreichend hohen Testqualität auszugehen. Dennoch wurden Reliabilitäts- und Validitätsanalysen durchgeführt, um die Qualität des Fachwissenstests zusätzlich zu überprüfen. Sie werden im Folgenden dargestellt. 
Reliabilität: Eine Reliabilitätsanalyse ergibt für die Fachwissensposttests aller Schüler einen Wert von Cronbachs $\alpha=.718$, was einem akzeptablen Wert entspricht. Die beiden Multiple-Choice-Aufgaben $A_{1}$ und A2 weisen niedrige Item-Skala-Korrelationen auf $\left(r_{\mathrm{A}_{1}}=.080\right.$ und $\left.r_{\mathrm{A} 2}=.101\right)$, so dass sich die Reliabilität des Fachwissenstests auf Cronbachs $\alpha=.722$ erhöhen würde, ließe man diese beiden Aufgaben außer Acht. Die Verbesserung fällt jedoch so gering aus, dass diese Maßnahme nicht geboten schien.

Validität: Um die Validität des Fachwissenstests zu beurteilen, wurde die inhaltliche Konvergenz und Diskriminanz bezüglich anderen Konstrukten bestimmt. Da es, wie in Kap. 2.2.4 gesehen, einen Zusammenhang zwischen schulischer Leistung und Motivation (Schiefele \& Schreyer 1994), Interesse (Hoffmann et al. 1998) und Selbstkonzept (Valentine et al. 2004) gibt, sollte ein Fachwissenstest, der die schulische Physikleistung abbilden will, mit diesen Variablen für das Fach Physik hoch korrelieren. Außerdem sollte er hoch mit der Schulnote Physik korrelieren sowie der Schulnote Mathematik, weil im Fachwissenstest auch mathematische Fertigkeiten gebraucht werden. Aus Tab. 6 wird ersichtlich, dass die Korrelationen zwischen dem Posttestergebnis und den genannten Variablen (post), die über den Fragebogen erhoben wurden, positiv und signifikant sind.

Umgekehrt sollte das Ergebnis des Fachwissenstests nicht mit Variablen korrelieren, die inhaltlich nicht mit ihm verwandt sind (diskriminante Validität), etwa dem Geschlecht, dem Fachinteresse an Deutsch und Biologie, der Schulzugehörigkeit und der unterrichtenden Lehrkraft. Aus der unteren Zeile von Tab. 6 wird deutlich, dass alle diese Variablen nicht signifikant mit dem Ergebnis des Fachwissenstests (post) korrelieren.

Zusammenfassend ist festzuhalten, dass der Fachwissenstest, der für diese Studie konzipiert wurde, trotz seiner Nichtstandardisierung ein hinreichend reliables und valides Instrument darstellt, den Lernstand der Schüler im Bereich der elektromagnetischen Induktion und des elektromagnetischen Schwingkreises in der 11. Jahrgangsstufe zu messen. Der Lernzuwachs, den jeder Schüler zwischen Post- und Prezeitpunkt ausbildet, wird auf einen Gain Score zwischen Post- und Pretest-Ergebnis abgebildet. Er ist ein Maß für die Lernleistung des individuellen Schülers.

Tab. 6. Konvergente und diskriminante Validität: Korrelationen des Fachwissenstests (post) mit erhobenen Variablen

\begin{tabular}{|c|c|c|c|c|c|}
\hline & $\begin{array}{c}\text { Motivation } \\
\text { Physik (post) }\end{array}$ & $\begin{array}{l}\text { Fachinteresse } \\
\text { Physik (post) }\end{array}$ & $\begin{array}{l}\text { Selbstkonzept } \\
\text { Physik (post) }\end{array}$ & $\begin{array}{l}\text { Physiknote } \\
\text { (Jgst. 11/1) }\end{array}$ & $\begin{array}{c}\text { Mathematiknote } \\
\text { (Jgst. 11/1) }\end{array}$ \\
\hline $\begin{array}{l}\text { Fachwissens- } \\
\text { test (post) }\end{array}$ & $\begin{array}{c}r=.45 \\
p<.001\end{array}$ & $\begin{array}{c}r=.52 \\
p<.001\end{array}$ & $\begin{array}{c}r=.55 \\
p<.001\end{array}$ & $\begin{array}{c}r=.75 \\
p<.001\end{array}$ & $\begin{array}{c}r=.60 \\
p<.001\end{array}$ \\
\hline
\end{tabular}

\begin{tabular}{|c|c|c|c|c|c|}
\hline & Geschlecht & $\begin{array}{l}\text { Fachinteresse } \\
\text { Deutsch (post) }\end{array}$ & $\begin{array}{l}\text { Fachinteresse } \\
\text { Biologie (post) }\end{array}$ & Schule & Lehrkraft \\
\hline $\begin{array}{l}\text { Fachwissens- } \\
\text { test (post) }\end{array}$ & $\begin{array}{l}r=-.03 \\
p=.744\end{array}$ & $\begin{array}{l}r=-.12 \\
p=.149\end{array}$ & $\begin{aligned} r & =.02 \\
p & =.881\end{aligned}$ & $\begin{aligned} r & =.05 \\
p & =.563\end{aligned}$ & $\begin{array}{c}r=.07 \\
p=.406\end{array}$ \\
\hline
\end{tabular}


Anders als in sonstigen schriftlichen Leistungserhebungen im Physikunterricht der Oberstufe war als Hilfsmittel lediglich der Taschenrechner, nicht jedoch die übliche Formelsammlung zugelassen. Mit dem reinen erinnerungsgestützten Rückgriff sollten die Schüler demonstrieren, inwiefern sie die relevanten Formeln verinnerlicht haben.

\subsubsection{Fragebogen}

Um die affektiven und andere individuelle Lernmerkmale zu erheben, wurde ein Fragebogen erstellt, der im Pretest beider Gruppen aus 82 Items besteht (s. Anhang 4). 71 der Items stammen dabei aus den PISA-2015-Fragebögen (OECD, Organisation for Economic Co-operation \& Development 2016), deren inhaltlicher Schwerpunkt die Naturwissenschaften waren. Es wurden dabei Skalen aus der Haupterhebung (Main Survey) sowie den Vorerhebungen (Field Trial) entnommen. Alle übrigen Items wurden selbsterstellt. Die PISA-Erhebung ist ausgerichtet auf 15-jährige Schüler, also Schüler der Mittelstufe, ist aber, was die Lernmerkmale angeht, auch auf ältere Schüler anwendbar. Die Skalen des PISA-2015-Fragebogens besitzen aufgrund ihrer intensiven Erprobung und iterativen Verbesserung eine hohe Güte, weshalb sie in der Erstellung des Fragebogens Einsatz fanden. Über die Fragebogennummern ließen sich die Schüler den Schulen, Kursen und Lehrkräften zuordnen. Die Schüler kennzeichneten den Fachwissenstest und den Fragebogen zu beiden Erhebungszeitpunkten mit einer pseudonymen Teilnehmerkennung, die sie nach einer allgemeinen Vorschrift aus nur ihnen bekannten persönlichen Daten generierten.

Der Fragebogen besteht aus drei Teilen, einem allgemeinen Frageteil, einem physik- und unterrichtsspezifischen Teil und einem informations- und kommunikationstechnologischen Teil. Nachfolgend werden in Tab. 7 die in der Studie verwendeten Variablen und ihre internen Reliabilitäten aufgeführt.

Die Reliabilitätswerte sind fast allesamt im akzeptablen bis guten Bereich, wie es von den Skalen des PISA-Tests zu erwarten war. Die Werte für die Konstrukte Begeisterung für digitale Geräte ( $\alpha=.646)$ und Lernorganisation ( $\alpha=.650$ ) sind kritisch, so dass sie für sich genommen nicht sehr reliabel sind. Die drei Konstrukte Leistungsmotivation, Lernorganisation sowie Prokrastination (in invertierter Form, das bedeutet o: starke Prokrastination, 3: schwache Prokrastination), aber auch die Hausaufgabendisziplin sind Indikatoren für die dispositionale Arbeitshaltung eines Schülers (Wagner \& Spiel 2002). Der Begriff der Arbeitshaltung wird hier verwendet als die Fähigkeit, schulische Lern- und Leistungsziele über einen längeren Zeitraum konsequent zu verfolgen und in Lernprozesse selbstregulativ zu investieren. Diese Definition ist allgemeiner als die von Keller \& Thiel (1998), nach der die Arbeitshaltung »die grundsätzliche Bereitschaft des Schülers zum pflichtbewussten, konzentrierten und gründlichen Lernen und Problemlösen « ist (Keller \& Thiel 1998, S. 5). Innerhalb der Arbeitshaltung sind die Konstrukte Leistungsmotivation, Lernorganisation, inverse Prokrastination und Hausaufgabendisziplin Facetten, ohne sie vollständig zu bestimmen. Die Hausaufgabendauer wird nicht zur Arbeitshaltung hinzugenommen, weil sie ein unspezifisches Maß für die Arbeitshaltung ist und gerade bei sehr kleinen und sehr großen Werten nicht eindeutig zu interpretieren ist. 
Tab. 7. Variablen des Pretest-Fragebogens, ihre Quellen und Reliabilitäten

\begin{tabular}{|c|c|c|c|c|c|}
\hline Variable & $\begin{array}{c}\text { Nummer } \\
\text { im Frage- } \\
\text { bogen }\end{array}$ & $\begin{array}{c}\text { Anzahl } \\
\text { der Items }\end{array}$ & $\begin{array}{l}\text { Anzahl der } \\
\text { Antwort- } \\
\text { stufen }\end{array}$ & $\begin{array}{c}\text { Nummer } \\
\text { im PISA- } \\
\text { Test }\end{array}$ & $\begin{array}{c}\text { Cron- } \\
\text { bachs a } \\
\text { (pre) }\end{array}$ \\
\hline
\end{tabular}

\section{Allgemeiner Teil}

\begin{tabular}{|l|lllll|}
\hline Geschlecht & 2.1 & 1 & 2 & SToo4 & n. a. \\
\hline Muttersprache & 2.3 & 1 & 3 & $-{ }^{10}$ & n. a. \\
\hline $\begin{array}{l}\text { Leistungsmotivation } \\
\text { Lernorganisation }\end{array}$ & 4. & 4 & 4 & ST1199 & .783 \\
\hline Prokrastination, invers & 5. & 5 & 4 & STo89 $^{11}$ & .650 \\
\hline
\end{tabular}

Physik- und unterrichtsbezogener Teil

\begin{tabular}{|c|c|c|c|c|c|}
\hline Selbstkonzept Physik & 8. & 5 & 4 & $\mathrm{ST} 130^{11}$ & .927 \\
\hline Motivation Physik & 9. & 4 & 4 & STo9 $4^{9}$ & .877 \\
\hline Fachinteresse Physik (zusätzlich D, M, Ch, B) & 11. & 5 & 5 & STog6 $6^{11}$ & n. a. \\
\hline Lehrerunterstützung Physik & 12. & 5 & 4 & $\mathrm{ST}_{100^{9}}$ & .753 \\
\hline Hausaufgabendisziplin Physik & 13.1 & 1 & 4 & $-{ }^{10}$ & n. a. \\
\hline Hausaufgabendauer Physik & 13.2 & 1 & 4 & $-{ }^{10}$ & n. a. \\
\hline Physiknote 10. Jgst. (echte Note) & $13 \cdot 3$ & 1 & 6 & $--^{10}$ & n. a. \\
\hline Physiknote 11/1 (in Punkten) & 13.4 & 1 & 17 & $-{ }^{10}$ & n. a. \\
\hline Selbstnote Physik über letzte 2 Monate (in Punkten) & 13.5 & 1 & 16 & -10 & n. a. \\
\hline Mathematiknote 11/2 (in Punkten) & 13.6 & 1 & 17 & $-{ }^{10}$ & n. a. \\
\hline Physik als Abiturfach geplant & 13.7 & 1 & 3 & $-{ }^{10}$ & n. a. \\
\hline
\end{tabular}

Teil über Nutzung digitaler Medien

\begin{tabular}{|l|cccccc|}
\hline Bisherige private Nutzung von Lernvideos & 15.3 & 1 & 4 & $-{ }^{10}$ & n. a. \\
\hline $\begin{array}{l}\text { Bisherige Nutzung von Lernvideos als Hausaufgaben } \\
\text { Begeisterung für digitale Geräte }\end{array}$ & 15.4 & 1 & 3 & $-{ }^{10}$ & n. a. \\
\hline Selbstkonzept digitale Kompetenz & 16. & 6 & 4 & IC013 & .646 \\
\hline
\end{tabular}

9 Die Skalen SToo4, ST119, STo94, ST100, ICo13 und ICo14 entstammen der Main Survey der PISA-2015-Erhebungsrunde.

10 Dieses Item wurde vom Autor erstellt.

11 Die Skalen STo87, STo89, ST130 und STo96 entstammen dem Field Trial des PISA-2015-Erhebungsrunde, der wesentlich umfangreicher war als der spätere Main Survey. Die Übersetzung der Items dieser Skalen erfolgte durch den Autor. 
Zwar korreliert die Hausaufgabendauer von Gymnasialschülern der 10. Jahrgangsstufe nach Spiel et al. (2002) signifikant mit der Arbeitsgenauigkeit $(r=.30)$ und der Ausdauer $(r=.20)$, aber eine lange Hausaufgabendauer kann auch Ausdruck von wenig zielgerichtetem Arbeiten aufgefasst werden. Aus diesem Grund bildet im Rahmen dieser Arbeit lediglich die Hausaufgabendisziplin die hausaufgabenbezogene Facette der Arbeitshaltung.

Des Weiteren ist der Reliabilitätswert für das Konstrukt Begeisterung für digitale Geräte mit $\alpha=.649$ nicht zufriedenstellend. Das Konstrukt wird im Zusammenhang eines möglichen Geschlechtereffekts (vgl. Kap. 6.3.2) verwendet.

Der Fragebogen zum Postzeitpunkt ist gekürzt um die stabilen Personenmerkmale des allgemeinen Teils sowie um die Angaben zu vergangenen Schulleistungen. Er besteht aus 31 Items (s. Anhang 5). Für den Postfragebogen wurde noch eine Skala entwickelt, die das themenspezifische Selbstkonzept für das Thema Induktion und elektromagnetischer Schwingkreis misst. Da es wenig sinnvoll erschien, die Ausbildung eines regelrechten Selbstkonzepts in diesem Bereich zu erheben, bevor die Themenreihe unterrichtet wurde, fand diese Skala nur im Posttest Anwendung. Hier gibt es einen Unterschied zum Fachwissenstest, der in identischer Form auch vor der Unterrichtsreihe eingesetzt wurde, um den tatsächlichen Lernzuwachs zwischen Pre- und Postzeitpunkt zu messen. Dabei findet das Vorwissen der Schüler Berücksichtigung, das jedoch im Wesentlichen aus partiellen Wissenselementen der 9. Jahrgangsstufe besteht, die die Schüler möglicherweise noch abrufen können. Das Selbstkonzept jedoch ist die komplexe Vernetzung mentaler Repräsentationen des eigenen Wissens und Könnens, dessen Ausbildung zum Prezeitpunkt nicht angenommen werden kann. Deshalb wurde das themenspezifische Selbstkonzept Induktion lediglich zum Postzeitpunkt erhoben.

Der Posttestfragebogen der Treatmentgruppe war identisch mit dem der Kontrollgruppe, doch wurden zusätzliche Items entwickelt, mit denen in geschlossener Form Rückmeldungen der Schüler zum Flipped Classroom erhoben wurden (s. Anhang 6). Die inhaltliche Gestaltung der Items orientierte sich an vereinzeltem mündlichen Feedback, das die Schüler im Verlauf der Unterrichtsreihe gegeben hatten. Um ihnen am Ende der Unterrichtsreihe aber auch die Möglichkeit zum offenen Feedback zu geben, wurden die Schüler gebeten, zusätzlich eine freie, stichpunktartige Rückmeldung zur Unterrichtsmethode zu geben, die ebenfalls ausgewertet wurde (s. Anhang 7). Einige der Variablen des Fragebogens sollen nun noch näher erläutert werden.

Muttersprache: Dieses Item wurde entwickelt, um mögliche Sprachschwierigkeiten zu berücksichtigen, die die Schüler der Treatmentgruppe mit den Lernvideos haben könnten. Falls signifikante Lernerfolgsunterschiede bei Schülern mit anderer Muttersprache auftreten sollten, könnten hier Ursachen zu finden sein.

Fachinteresse: Der Stamm der Items fordert die Schüler dezidiert auf, sich bei den Angaben auf die letzten beiden Monate zu beschränken. Das bedeutet, es geht nicht um das Interesse am Fach, wie die Schüler es bisher in ihrer gesamten Schulzeit erlebt haben oder wie sie 
es aktuell erleben, sondern wie sehr sie an diesem Fach in den letzten zwei Monaten interessiert waren. Auf einer vierstufigen Likertskala können die Schüler ihr Gesamtinteresse am Unterrichtsfach angeben, das - wie in Kap. 2.2.2 - ein mehrfaktorielles Konstrukt ist und Sachinteresse, situationales Interesse, soziales Lernklima und Unterrichtsgestaltung beinhaltet. Der Hintergrund ist, dass sich die Interessensabfrage im Posttest auf die Zeit der Unterrichtsreihe beziehen soll, wobei der Ausdruck »zwei Monate« die jüngere Vergangenheit bezeichnen soll, weniger die kalendarische Zeitspanne. Der Itemstamm der Konstrukte Selbstkonzept und Motivation besitzt nicht die zeitliche Beschränkung auf die letzten beiden Monate, weil die Schüler hier eine Selbsteinschätzung vornehmen sollen, während die Angabe des Fachinteresses eher eine subjektive In-Bezug-Setzung zum Unterricht, den Themen, den Methoden, dem Lehrerverhalten und weiteren Faktoren in den letzten zwei Monaten darstellt.

Lehrerunterstützung: Wie beim Fachinteresse sind auch hier die Schüler aufgefordert, eine Einschätzung über den Zeitraum der letzten zwei Monate zu treffen. Nur so lässt sich für die Forschungshypothese $\mathrm{H}_{3}$ a beurteilen, ob die von den Schülern wahrgenommene Lehrerunterstützung im Flipped Classroom besser wurde oder nicht. Die Items dieser Skala lauten: »Unser Physiklehrer interessiert sich für den Lernfortschritt jedes einzelnen Schülers«, »Unser Physiklehrer unterstützt uns zusätzlich, wenn wir Hilfe brauchen «, »Unser Physiklehrer unterstützt uns beim Lernen «, »Unser Physiklehrer erklärt uns etwas so lang, bis wir es verstehen « und »Unser Physiklehrer gibt uns Gelegenheit, unsere Meinung zu sagen.« Für die Kontrollgruppe wird erwartet, dass es keinen Unterschied zwischen Pre- und Posttestzeitpunkt gibt, denn das Lehrerverhalten war in den Kursen der Kontrollgruppe nicht anders als zuvor.

Hausaufgabendisziplin und -dauer: Auch diese Items sind von den Schülern für die jeweils letzten zwei Monate zu beantworten. Die vier Antwortstufen für die Hausaufgabendisziplin (»Wie regelmäßig haben Sie in den letzten 2 Monaten die Physikkausaufgaben erledigt?» - Selten/nie, hin und wieder, häufig, immer) und die Hausaufgabendauer (»Wie viel Zeit haben Sie während der letzten 2 Monate durchschnittlich für eine Physikhausaufgabe investiert? o-5 min, 5-10 min, 10-15 min, 15-30 min) wurden von einem Expertenkreis erfahrener Physiklehrer geprüft. Für die Hausaufgabendauer wurde der geschlossenen Form gegenüber der offenen Freitextform der Vorzug gegeben, um den Schülern durch Zeitkategorien Orientierung beim Ausfüllen zu geben. Die beiden Items wurden zur Erprobung in zwei Physikkursen der 12. Jahrgangsstufe getestet. Dabei gab es keine Antwortstufe, die mehr als $40 \%$ der Schüler wählten oder weniger als 10\% der Schüler nicht wählten. Deshalb wurden alle Antwortstufen in ihrer Form beibehalten.

Physiknote 10. Jgst. und 11/1 und Mathematik 11/1: Zeugnisnoten als Maß für die fachbezogene Schulleistung sind bei aller Kritik an ihrer Objektivität und Reliabilität ökonomisch und valide, auch wenn die Vergleichbarkeit über Lerngruppen hinweg ein Problem darstellt (Tent 2006). Zwischen der pseudonymen Selbstauskunft über eigene Schulnoten und den tatsächlich erzielten Noten gibt es allgemein keinen Unterschied. Helmke (1992, S. 89f) stellt klassenbasierte Korrelationen von $r=.75$ bis $r=.97$ fest.

Selbstnote Physik über die letzten zwei Monate: Mit dem Item »Wie viele Punkte würden Sie sich im Fach Physik für den Zeitraum der letzten zwei Monate selbst geben? «sollen die 
Schüler eine Selbstbewertung ihrer Leistung vornehmen, die sich dem Selbstkonzept zurechnen lässt. Wenn es hier signifikante Unterschiede in den Mittelwertsänderungen zwischen den Gruppen geben sollte, liegt eine Beziehung zur jeweiligen Unterrichtsform nahe.

Sämtliche andere Konstrukte dienen zu überprüfen, ob die Lernausgangslage für Kontrollund Treatmentgruppe oder - wenn es um mögliche Geschlechtereffekte geht - für Mädchen und Jungen vergleichbar ist. Interessant sind dabei die Begeisterung für digitale Geräte und das Selbstkonzept digitale Kompetenz. Es ist anzunehmen, dass die Jungen hier eine höhere Selbsteinschätzung haben als Mädchen (Christoph et al. 2015). Ein Geschlechtereffekt bei Jugendlichen konnte aber in den IT-Basisfähigkeiten, zu denen das Ansehen von Lernvideos zweifelsfrei gehören, durch empirische Forschung bisher nicht festgestellt werden.

\subsection{Auswertungsmethoden}

Im quasiexperimentellen Design, wie es für diese Studie verwendet wird, sind die Daten hierarchisch geclustert. Alle Schüler befinden sich in einem Kursverbund, alle Kurse gehören zu einer Schule. Die Veränderungen, die in einem Kurs unter einem Lehrer auftreten, müssen nicht mit dem Treatment zusammenhängen, sondern können auch mit dem Cluster zusammenhängen. So gibt es die Subjektebene, die Kursebene, die Lehrerebene und Schulebene. Das statistische Verfahren, hierarchisch geclusterte Daten auszuwerten, ist die Mehrebenenanalyse (Eid et al. 2010, S. 699ff). Wenn die Schulebene nicht berücksichtigt wird, besitzen die Daten eine Drei-Ebenen-Struktur (Schüler, Kurs, Lehrer). Die Schwierigkeit bei der Mehrebenenanalyse ist, den Anforderungen der Stichprobengröße gerecht zu werden. Auf der höchsten Ebene ist eine Zahl von 5o Einheiten empfohlen, als Minimum sind zehn Einheiten notwendig, um Cross-Level-Interaktionen analysieren zu können (Eid et al. 2010, S. 715; Kuhn 2014). Im vorliegenden Fall würde das zehn verschiedene Lehrkräfte bedeuten, was nicht realisiert werden konnte.

Um Gruppenvergleiche im Zweigruppen-Pre-/Postplan vorzunehmen, ist die zweifaktorielle Varianzanalyse (ANOVA) mit Messwiederholung auf einem Faktor die Methode der Wahl (Döring \& Bortz 2016, S. 739). Wenn mehrere miteinander korrelierende abhängige Variablen zugleich betrachtet werden sollen, wird eine multivariate Varianzanalyse (MANOVA) mit Messwiederholung angewendet. In der empirischen Bildungsforschung ist die Varianzanalyse das am häufigsten eingesetzte Auswertungsverfahren (Keselman et al. 1998). Die nominellen Anforderungen, um eine ANOVA durchzuführen, sind Normalverteilung, Varianzenhomogenität, Intervallskalierung der abhängigen Variablen und Nominalskalierung der unabhängigen Variablen. Der Vorteil der ANOVA ist, dass sie - mit Einschränkungen - robust gegenüber Verletzungen der Normalverteilung und Varianzenhomogenität ist (Field 2013, S. 444f). Gerade in quasiexperimentellen Designs ist Normalverteilung nicht immer gewährleistet. Sind die Größen der Gruppen oder Untergruppen nicht gleich, gewinnt jedoch die Varianzenhomogenität an Bedeutung. Im hier verwendeten Versuchsdesign, in dem die Zellen nicht alle gleichmäßig besetzt sind, ist diese Voraussetzung daher immer mit dem Levene-Test zu prüfen. 
Statt zweifaktorieller ANOVAs mit Messwiederholung auf einem Faktor werden in der Auswertung einfaktorielle ANOVAs der Gain Scores angewendet. Hierbei wird der Zuwachs einer Variable zwischen Pre- und Posttest auf eine Gain Score-Variable abgebildet. Sie ist üblicherweise lediglich die Differenz von Post- und Pretestwert. Der Gain Score wird dann als abhängige Variable einer einfaktoriellen ANOVA verwendet. Die Ergebnisse beider Analyseverfahren sind absolut identisch (Dimitrov \& Rumrill Jr 2003).

Für den Lernzuwachs soll der normierte Gain Score g verwendet werden, weshalb sich die einfaktorielle ANOVA der Gain Scores auch statistisch anbietet. Der normierte Gain Score $g$ wurde von Hake (1998) in Pre-/Postfachwissenstests eingeführt als Quotient von tatsächlichem Lernzuwachs zu maximal möglichem Lernzuwachs und kann einen Wert zwischen o und 1 annehmen:

$$
g=\frac{\text { post score-pre score }}{\text { max score-pre score }} \quad \begin{aligned}
& \text { pre score: } \text { Punktzahl im Pretest } \\
& \text { post score: Punktzahl im Posttest } \\
& \text { max score: Maximal mögliche Punktzahl im Test }
\end{aligned}
$$

Ursprünglich genutzt, um Testergebnisse des Force Concept Inventory (FCI) schul- und landesübergreifend vergleichen zu können, wird Hakes g zunehmend auch in anderen leistungsbezogenen Kontexten verwendet. Wo liegt der Vorteil bei Hakes g? Bei der reinen Differenz der Punktezahl von Posttest und Pretest macht es keinen Unterschied, ob Zuwächse am oberen oder unteren Ende der Punkteskala stattfanden. Beispielsweise berücksichtigt ein Differenz-Gain Score von 10 Punkten nicht, ob das Vorwissen gering oder hoch war. In der schulischen Praxis sind Leistungserhebungen aber so gestaltet, dass es schwieriger ist, am oberen Ende der Skala Zuwächse zu erzielen als am unteren Ende. Zwar werden in der Schulpraxis keine Pretests verwendet, aber die Notengebung in Klausuren und Tests beruht darauf, dass die Notenleistung nicht proportional zur erreichten Zahl der Punkte beziehungsweise Bewertungseinheiten ist. Für ein »mangelhaft « (1 Punkt), muss ein Schüler bereits $20 \%$ der Bewertungseinheiten erzielt haben, ansonsten erhält er ein »ungenügend «. Für ein »ausreichend « (5 Punkte) sind bereits 50\% der Bewertungseinheiten notwendig. Das bedeutet, um das untere Drittel der Notenskala (o-15 Punkte) zu verlassen, muss ein Schüler mehr als die Hälfte der Bewertungseinheiten erreichen. Im Vergleich dazu verändert sich die Benotung im mittleren und höheren Bereich nur noch in $5 \%$-Intervallen. Schulische Leistungserhebungen enthalten Aufgaben unterschiedlichen Anforderungsniveaus. Die Leistungsdifferenzierung, die sich in der Notendifferenzierung widerspiegelt, erfolgt durch diese Aufgabenniveaus. Leistungsschwächere Schüler werden selten die schwierigen Transferaufgaben erfolgreich lösen können, deren Bewertungseinheiten notwendig sind, um in den oberen Bereich der Notenskala vorzustoßen. Der Vorteil des normierten Gain Score $g$ von Hake ist es nun, dass in einem Pre/Posttest der Vorwissenstand und damit die individuelle Ausgangslage stärker ins Gewicht fällt. Normiert auf den jeweils maximal möglichen Lernzuwachs bedeutet das, dass ein Schüler mit einem hohen Pretestwert ein höheres $g$ erhält als ein Schüler mit einem niedrigen Prestestwert, auch wenn beide denselben Gewinn an Bewertungseinheiten erzielten. 
Weil der Fachwissenstest analog zu einer typischen Physikklausur mit Aufgaben unterschiedlichen Anforderungs- und Schwierigkeitsniveaus konzipiert wurde, bildet der normierte Gain Score g von Hake den Lernzuwachs jedes Schülers in dieser Studie besser ab.

Ein zusätzliches Argument, Hakes g als Variable für den Lernzuwachs zu verwenden, ist seine höhere Korrelation mit der Physiknote 11/1 der Kontrollgruppe. Im traditionellen Unterricht sollte der Lernzuwachs jedes Schülers, der durch den Fachwissenstest erhoben wird, mit seiner Physiknote 11/1 hoch korrelieren. Im traditionellen Unterrichtsformat sollte ein Schüler einen Lernzuwachs erzielen, der seiner in der Zeugnisnote indizierten Leistung entspricht. Zwischen der Physiknote 11/1 und dem absoluten Differenz-Gain Score $\Delta$ (post score - pre score) gibt es eine Korrelation von $\mathrm{r}=.569(\mathrm{p}<.001)$. Jedoch ist die Korrelation zwischen der Physiknote $11 / 1$ und Hakes $g$ mit $\mathrm{r}=.685(\mathrm{p}<.001)$ noch höher. Hakes $\mathrm{g}$ bildet in der traditionell unterrichteten Kontrollgruppe also noch besser eine den Physiknoten 11/1 der Schüler entsprechenden Lernleistung ab als $\Delta$. Aufgrund der höheren Reliabilität wird zur Messung des Lernzuwachses und damit der Lernleistung in dieser Studie Hakes $g$ als Messgröße verwendet.

Sämtliche anderen Gain Scores - etwa für Motivation, Fachinteresse oder Lehrerunterstützung - werden hingegen als Differenz-Gain Scores $\Delta$ in einfaktoriellen ANOVAs analysiert. Ein normierter Gain Score lässt sich für diese Variablen zum einen inhaltlich nicht begründen und zum anderen lässt er sich für die Schüler nicht berechnen, die bereits zum Pretestzeitpunkt den Höchstwert auf der Skala angegeben haben, da hier eine Division durch Null vorliegt.

Zur Untersuchung möglicher Moderatoreffekte mit zwei oder mehreren unabhängigen Variablen erfolgen die Berechnungen in mehrfaktoriellen ANOVAs. 


\section{Ergebnisse und Diskussionen}

Nachdem die Methoden und Instrumente dargestellt wurden, mit denen die Forschungsfragen beantwortet werden sollen, werden nun die Ergebnisse der Erhebungen vorgestellt und diskutiert. Dies geschieht entlang der Forschungsfragen. Zunächst wird jedoch die Stichprobe hinsichtlich der kognitiven und affektiven Lernausgangslage beschrieben.

\subsection{Ausgangslage: Deskriptive Statistik zur Stichprobe}

Insgesamt haben $\mathrm{N}=151$ Schüler an der Studie teilgenommen. Dies waren alle in den acht Physikkursen befindlichen Schüler. Für die einzelnen Kurse und Schulen ergibt sich eine Verteilung, die in Tab. 8 dargestellt ist.

Es ist ersichtlich, dass 30 Teilnehmer (20\% der Stichprobe) aus Schule B kamen. Bei der Auswertung muss berücksichtigt werden, dass hier ein Lehrerwechsel stattgefunden hat (vgl. Kap. 5.1.2). In der Treatmentgruppe hat also ein anderer Lehrer (Lehrer 5) unterrichtet als in der Kontrollgruppe (Lehrer 4). Alle Berechnungen, die die Rolle der Lehrkraft beim Methodenwechsel explizit berücksichtigen wollen, müssen dementsprechend ohne diesen Stichprobenteil vorgenommen werden und nur Schule A in den Blick nehmen.

Tab. 8. Kurs-/Gruppengrößen sowie Geschlechterverteilung

\begin{tabular}{|c|c|c|c|c|c|}
\hline & & $\begin{array}{c}\text { traditionell } \\
2015 / 16\end{array}$ & $\begin{array}{l}\text { flipped } \\
2016 / 17\end{array}$ & $\begin{array}{c}\text { Summe } \\
\text { pro Lehrer }\end{array}$ & $\begin{array}{c}\text { Summe } \\
\text { pro Schule }\end{array}$ \\
\hline \multirow{3}{*}{ Schule A } & Lehrer 1 & 22 & 16 & 38 & \multirow{3}{*}{121} \\
\hline & Lehrer 2 & 21 & 22 & 43 & \\
\hline & Lehrer 3 & 21 & 19 & 40 & \\
\hline \multirow[t]{5}{*}{ Schule B } & Lehrer 4/5 & 16 & 14 & 30 & 30 \\
\hline & $\begin{array}{c}\text { Summe } \\
\text { pro Gruppe }\end{array}$ & 80 & 71 & & \\
\hline & $\begin{array}{c}\text { Summe } \\
\text { insgesamt }\end{array}$ & \multicolumn{2}{|c|}{151} & & \\
\hline & $\begin{array}{l}\text { Geschlecht } \\
\text { pro Gruppe }\end{array}$ & $\begin{array}{l}\text { weiblich: } 17 \\
\text { männlich: } 63\end{array}$ & $\begin{array}{l}\text { weiblich: } 23 \\
\text { männlich: } 48\end{array}$ & & \\
\hline & $\begin{array}{l}\text { Geschlecht } \\
\text { insgesamt }\end{array}$ & \multicolumn{2}{|c|}{$\begin{array}{l}\text { weiblich: } 40 \\
\text { männlich: } 111\end{array}$} & & \\
\hline
\end{tabular}




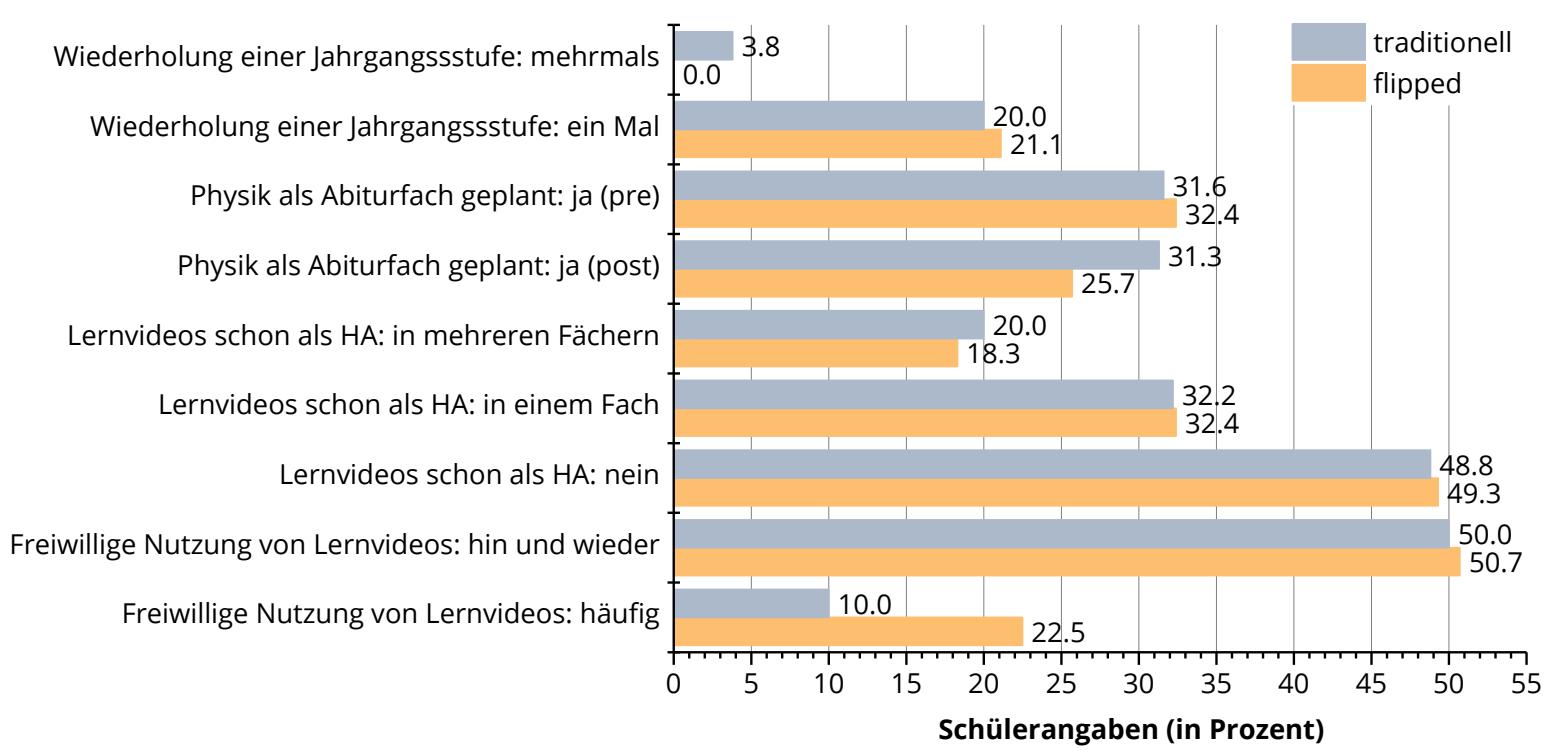

Abb. 13. Vergleich der Eigenschaften der Kontroll- und Treatmentgruppe

Die Schüler waren 16 bis 18 Jahre alt. Von allen 151 Teilnehmern gaben zwei an, dass Deutsch nicht ihre Muttersprache sei, und nur einer von ihnen war in der Treatmentgruppe, so dass dem Verständnis der Videos praktisch keine sprachlichen Hürden im Weg standen.

In fast allen Bereichen weisen Kontroll- und Treatmentgruppe vergleichbare Eigenschaften auf (vgl. Abb. 13). Die Quote der Schüler, die bereits eine Jahrgangsstufe wiederholt haben, ist fast gleich. Keiner der Schüler hat dabei jedoch die 11. Jahrgangsstufe wiederholt. ${ }^{12}$ Die Angabe, ob geplant ist, Physik als Abiturfach zu belegen, kann als weiterer Indikator für das Engagement im Fach Physik gewertet werden. Sowohl hier als auch bei der Frage, ob schon Erfahrungen mit Lernvideos als Hausaufgaben vorliegen, sind sich beide Gruppen sehr ähnlich. Lediglich bei der häufigen freiwilligen häuslichen Nutzung von Lernvideos, liegt der Anteil der Schüler in der Treatmentgruppe deutlich über dem der Kontrollgruppe, wie Abb. 13 zeigt. In der Treatmentgruppe machten 16 Schüler diese Angabe, in der Kontrollgruppe hingegen acht Schüler.

Um für die Studie die Lernausgangslage näher zu beschreiben, werden nun die abhängigen Variablen zum Pretestzeitpunkt dargestellt werden. Für den Gruppenvergleich wird eine univariate Varianzanalyse verwendet, um zu entscheiden, ob es hier signifikante Unterschiede zwischen Kontroll- und Treatmentgruppe gibt.

\subsubsection{Kognitive Lernausgangslage}

Die Lernwirksamkeit des Flipped Classroom, also sein Einfluss auf die Lernleistung, wird mittels des Fachwissenstests in Kontroll- und Treatmentgruppe erhoben. Die Notenangaben der

12 Diese zusätzliche Information gaben die Kursleiter. 
Tab. 9. Vergleich der Leistungsvariablen der Kontroll- und Treatmentgruppe zum Prezeitpunkt

\begin{tabular}{|c|c|c|c|c|c|c|c|c|}
\hline & \multicolumn{3}{|c|}{ traditionell } & \multicolumn{3}{|c|}{ flipped } & \multirow[b]{2}{*}{$F$} & \multirow[b]{2}{*}{$p$} \\
\hline & $N$ & $M$ & $S D$ & $N$ & $M$ & $S D$ & & \\
\hline Physiknote 11/1 (0-15) & 80 & 7.94 & 3.34 & 71 & 9.21 & 2.71 & 6.503 & .012 \\
\hline Physiknote 10. Jgst. (1-6) & 80 & 2.35 & .84 & 71 & 2.38 & .94 & .046 & .831 \\
\hline Mathematiknote 11/1 (0-15) & 80 & 9.66 & 3.17 & 71 & 9.96 & 2.75 & .358 & .550 \\
\hline
\end{tabular}

Schüler in den Fächern Physik und Mathematik werden verwendet, um vorab zu entscheiden, ob die Kurse in der Kontroll- und Treatmentgruppe überhaupt kognitiv vergleichbar sind.

In der Übersicht Tab. 9 fällt auf, dass die mittlere Physiknote im 1. Halbjahr der 11. Jahrgangsstufe in der Treatmentgruppe signifikant höher ausfällt als in der Kontrollgruppe. Wenngleich es einen signifikanten Unterschied zwischen Kontroll- und Treatmentgruppe gibt, so gibt es doch in den Physiknoten 11/1 keinen signifikanten Unterschied zwischen den acht Kursen, $F(7,142)=1.235, p=.288$. Alle acht Kurse sind dementsprechend homogen in ihrer Leistung. Da der Levene-Test nicht signifikant ausfällt $(p=.172)$, kann auch Varianzenhomogenität angenommen werden. Boxplots in Abb. 14 zeigen die Streuung und die Lagemaße der Physiknoten 11/1 der acht Kurse.

Es ist auffällig, dass die Mittelwerte der Physiknoten 11/1 aller vier Kontrollkurse unter, die Mittelwerte aller vier Treatmentkurse jedoch über dem Gesamtmittelwert aller Schüler liegen. Besonders Kurs $\mathrm{T}_{5}$ sticht heraus, in dem lediglich ${ }_{14}$ Schüler sind. Sie erzielten im Vergleich zu den anderen Kursen die besten Halbjahresleistungen. Die Kursunterschiede sind jedoch, wie oben erwähnt, nicht signifikant. Wenn später die Rolle der Lehrkraft in der Studie berücksichtigt wird, muss auf die 30 Teilnehmer der Schule B verzichtet werden, da es hier zwischen Kontroll- und Treatmentjahrgang einen personellen Wechsel (Lehrer 4 und 5) gegeben hat.

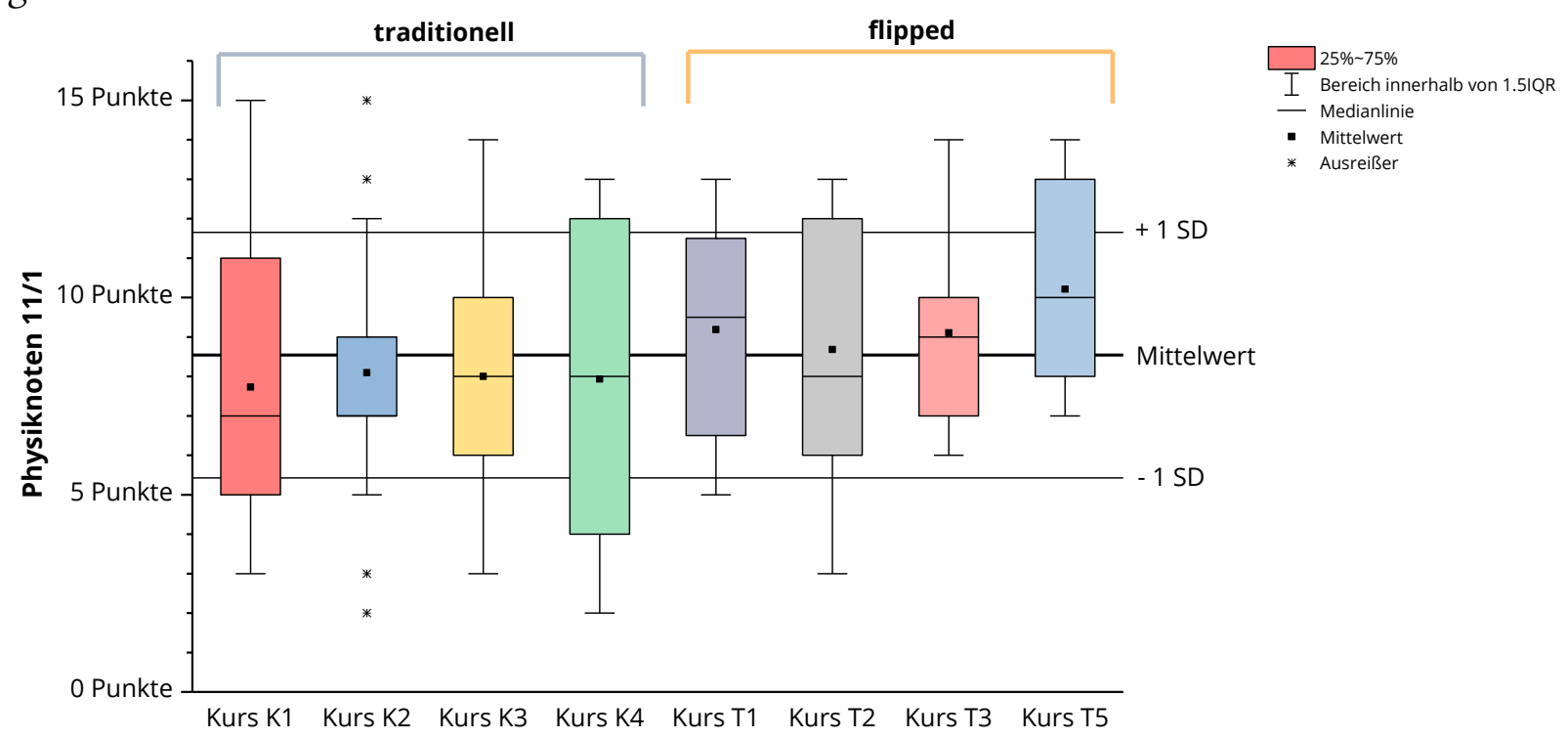

Abb. 14. Boxplots der Physiknoten 11/1 aller Kurse im Fach Physik. Kursbezeichnung: Buchstabe: $K=$ Kontrollgruppe, T = Treatmentgruppe; Ziffer: 1-5 Lehrervariable 
Da die Lehrer für die Vergabe der Halbjahresnoten Verantwortung tragen und drei der vier Lehrer sowohl Kontroll- als auch Treatmentkurse unterrichtet haben, ist zu überprüfen, ob es einen Zusammenhang zwischen der Höhe der Noten und der Lehrkraft gibt. Eine ANOVA mit Physiknote 11/1 als abhängiger und der Lehrkraft als unabhängiger Variable zeigt jedoch, dass es keinen signifikanten Lehrereinfluss auf die Note gibt, $F(4,145)=1.225, p=.303$. Mithin ist die Tatsache, dass die Mittelwerte der Physiknoten 11/1 in der Treatmentgruppe höher ausfallen als in der Kontrollgruppe, auf keinen systematischen Zusammenhang zurückzuführen.

Während der Notenmittelwert aller Schüler am Ende der 10. Jahrgangsstufe bei $M_{10}$. fgst $=2.36, S D=.89 \mathrm{lag}$, betrug er ein halbes Jahr später $M_{11}$. fgst $=2.79, S D=1.11$ (umgerechnet von der 0-15-Punkteskala auf die 6-bis-1-Notenskala). Das entspricht einem signifikanten Notenabfall, $F(1,140)=39.051, p<.001$. Das bedeutet, dass die Schüler, die in der 11 . Jahrgangsstufe Physik belegt hatten, noch in der 10. Jahrgangsstufe signifikant bessere Noten hatten. Der Effekt des Übergangs von der 10. in die 11. Jahrgangsstufe ist mit $\eta_{p}{ }^{2}=.218 \mathrm{sehr}$ groß. Die Physikhalbjahresnoten der 11. Jahrgangsstufe werden in dieser Studie als Variable für das Leistungsniveau im Fach Physik verwendet. Die Forschungshypothese Hza prüft, ob das Leistungsniveau im Fach Physik die Wirkung des Flipped Classroom beeinflusst.

Zusammenfassend lässt sich sagen, dass die leistungsbezogene Lernausgangslage für alle Einzelkurse gleich ist, es also keine signifikanten Leistungsunterschiede zwischen den Kursen gibt, die sich in den Physiknoten manifestieren. Jedoch besitzen die vier Kurse der Treatmentgruppe im Mitteln eine höhere Physiknote als die Kurse der Kontrollgruppe. Die Lehrkräfte besitzen keinen signifikanten Einfluss auf die Höhe der mittleren Kursnoten.

\subsubsection{Affektive Lernausgangslage und Stützfaktoren}

Wie auch die kognitiven Voraussetzungen sollen nun die selbstbezogenen, affektiven Merkmale sowie die Stützfaktoren (Lehrerunterstützung, Hausaufgabendisziplin und -dauer) der Schüler in den jeweiligen Gruppen beschrieben werden. Die Variablenwerte werden in Tab. 10 gegenübergestellt und Abb. 15 grafisch veranschaulicht, eine ANOVA prüft, ob sich Kontroll- und Treatmentgruppe in diesen Variablen signifikant unterscheiden.

In allen affektiven Merkmalen - Motivation, Fachinteresse und Selbstkonzept - gibt es keine signifikanten Unterschiede. Für keine der Variablen ist der Levene-Test signifikant $(p>.05)$. Auch zwischen den einzelnen der acht Kurse gibt es keine signifikanten Unterschiede, $F_{\text {Mot }}(7,149)=1.017, p=.422 ; F_{\text {Fachint }}(7,149)=.584, p=.768 ; F_{S k}(7,149)=.959, p=.463$. Welcher Lehrer unterrichtet, hat ebenfalls keinen Effekt auf die Ausprägung der Merkmale, wie eine mehrfaktorielle ANOVA mit Lehrkraft als zweitem Faktor zeigt, $F_{M o t}(4,145)=.224, p=.924$; $F_{\text {Fachint }}(4,145)=1.184, p=.321 ; F_{S k}(4,145)=.111, p=.979$. 
Tab. 10. Mittelwerte der affektiven Merkmale der Kontroll-und Treatmentgruppe zum Prezeitpunkt

\begin{tabular}{|c|c|c|c|c|c|c|c|c|}
\hline & \multicolumn{3}{|c|}{ traditionell } & \multicolumn{3}{|c|}{ flipped } & \multirow[b]{2}{*}{$F$} & \multirow[b]{2}{*}{$p$} \\
\hline & $N$ & $M$ & $S D$ & $N$ & $M$ & $S D$ & & \\
\hline Motivation pre (0-3) & 80 & 1.69 & 69 & 71 & 1.52 & .74 & 2.040 & .155 \\
\hline Fachinteresse pre (0-3) & 80 & 1.96 & .79 & 71 & 1.90 & .74 & .240 & .625 \\
\hline Selbstkonzept pre (0-3) & 80 & 1.71 & .70 & 71 & 1.73 & .71 & .015 & .902 \\
\hline Lehrerunterstützung pre (0-3) & 80 & 1.88 & .52 & 71 & 2.07 & .50 & 4.953 & .028 \\
\hline HA-Disziplin pre (0-3) & 80 & 1.14 & .96 & 71 & 1.46 & .99 & 3.558 & .061 \\
\hline HA-Dauer pre (0-3) & 80 & 1.13 & .97 & 71 & 1.39 & .95 & 2.707 & .102 \\
\hline Begeisterung für digitale Geräte (0-3) & 79 & 1.96 & .42 & 71 & 1.99 & .47 & .135 & .714 \\
\hline Selbstkonzept digit. Kompetenz (0-3) & 79 & 2.09 & .56 & 71 & 1.99 & .56 & 1.168 & .281 \\
\hline
\end{tabular}

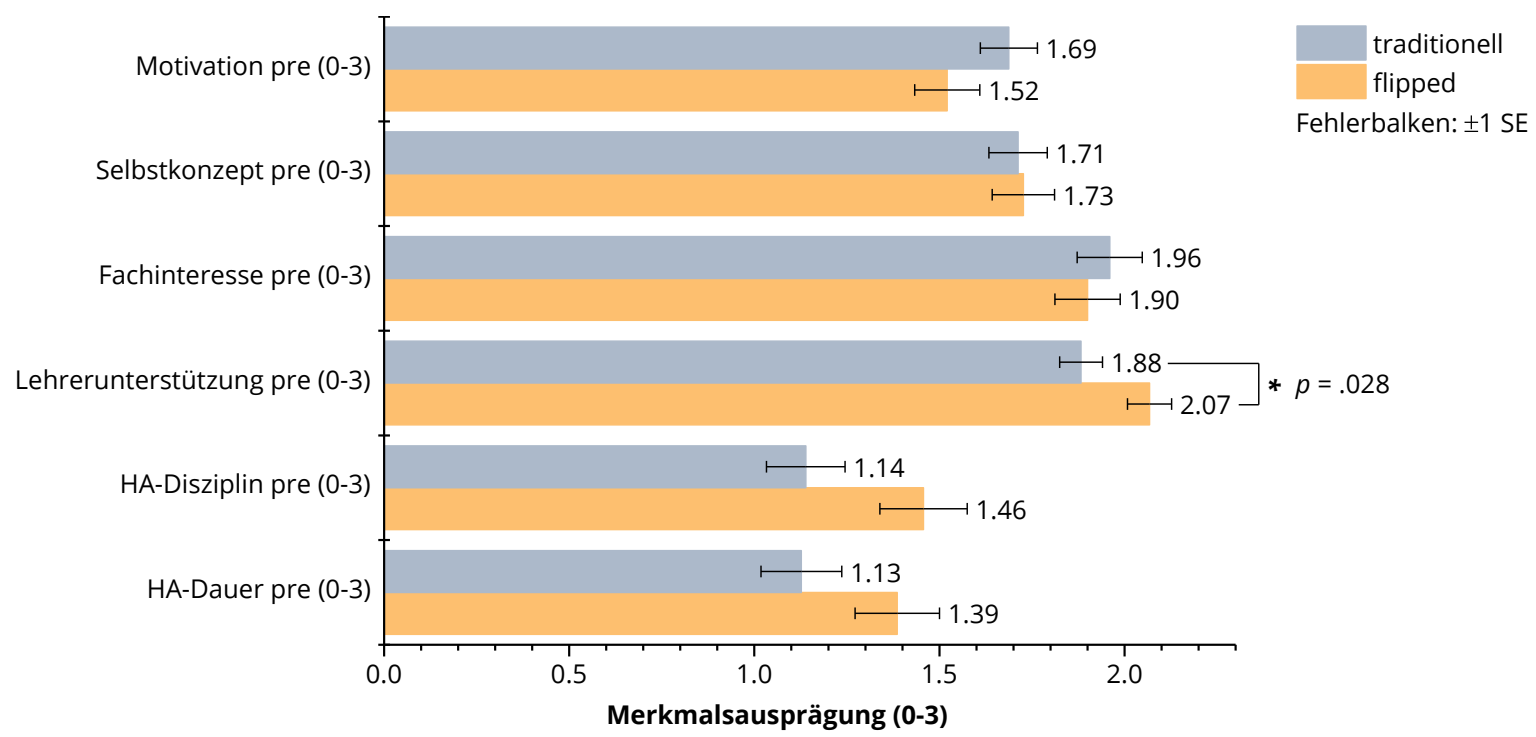

Abb. 15. Affektive Lernmerkmale und Stützfaktoren der Kontroll- und Treatmentgruppe zum Prezeitpunkt

Auffällig sind bei den Stützfaktoren allein die Unterschiede in der Lehrerunterstützung. Knapp nicht signifikant sind die Unterschiede in der Hausaufgabendisziplin. Beim Blick auf die Lagemaße und Streuung der Werte für die Lehrerunterstützung (Abb. 16) fallen vor allem drei Kurse auf, deren Gruppenmittelwerte deutlich über dem Gesamtmittelwert liegen, der Kurs $\mathrm{T}_{1}$ an Schule A und die beiden Kurse an Schule B ( $\mathrm{K}_{4}$ und $\mathrm{T}_{5}$ ).

Post-hoc-Vergleiche im Gabriel-Test zeigen, dass sich der Kursmittelwert in Lehrerunterstützung von Kurs $\mathrm{T}_{5}$ von denen aller anderen signifikant unterscheidet - mit Ausnahme der 


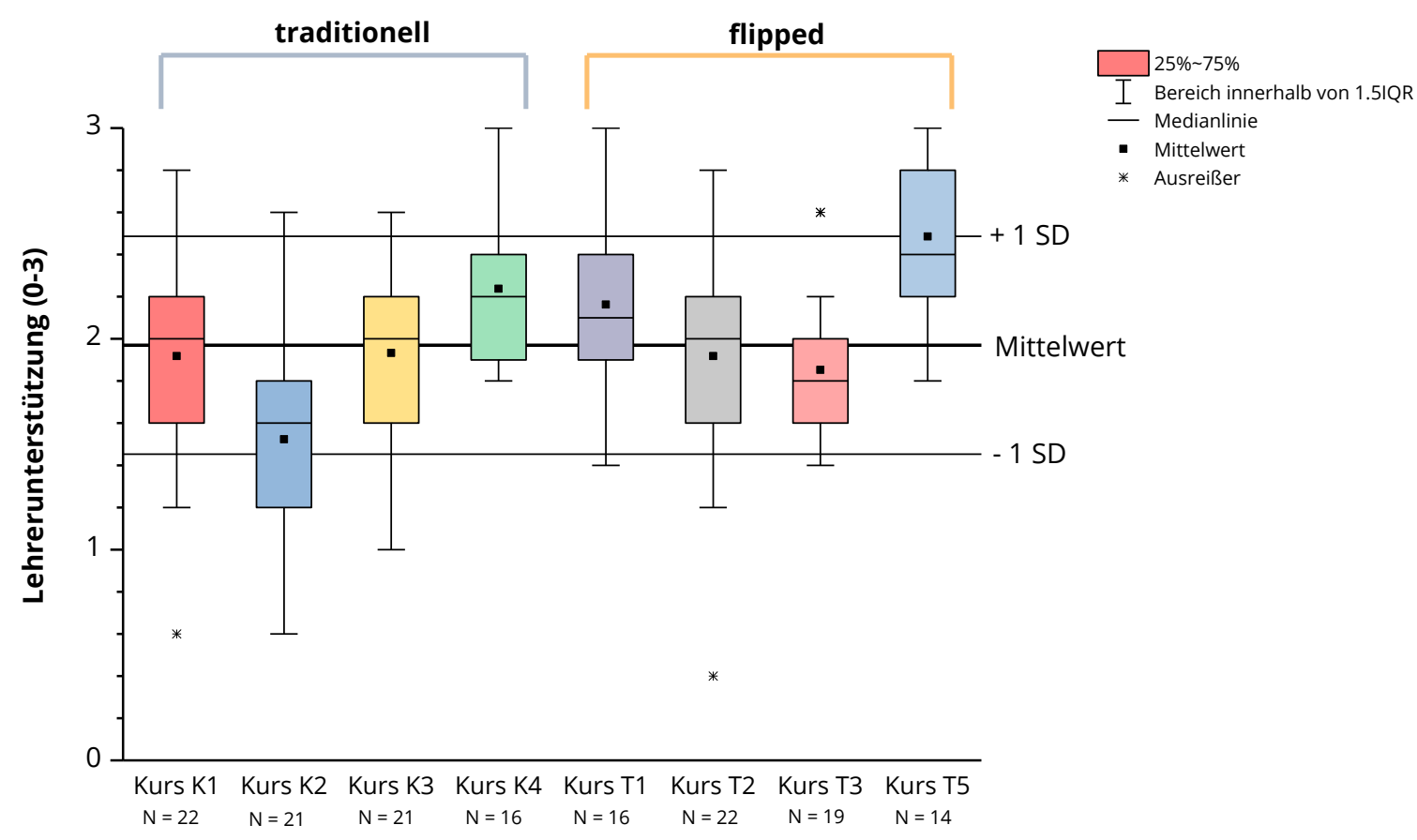

Abb. 16. Boxplots der Variable Lehrerunterstützung pre (o-3) Kursbezeichnung: Buchstabe: $K=$ Kontrollgruppe, T = Treatmentgruppe; Ziffer: 1-5 Lehrervariable

beiden Kurse $\mathrm{K}_{4}$ und $\mathrm{T}$. Alle drei Kurse haben im Vergleich zu den anderen eine deutlich geringere Anzahl an Schülern (Kurs K4: 16 Schüler, Kurs T1: 16 Schüler, Kurs T5: 14 Schüler), was dazu führen kann, dass dort ein intensiveres Betreuungsverhalten möglich ist und von den Schülern qualitativ anders erlebt wird. Des Weiteren könnten die Gruppenunterschiede mit den unterschiedlichen Lehrerpersönlichkeiten erklärt werden. Da die Lehrkräfte 4 und 5 der beiden Kurse $\mathrm{K}_{4}$ und $\mathrm{T}_{5}$ (beide an Schule B) jedoch jeweils nur einen Physikkurs unterrichtet haben, kann dies nicht überprüft werden. Ein Post-hoc-Vergleich der Gruppenmittelwerte von $\mathrm{K}_{1}$ und $\mathrm{T}_{1}$ (beide von Lehrer 1 unterrichtet) fällt jedoch nicht signifikant aus $(p=.947)$. Wenn bei späteren Analysen der Lehrerfaktor berücksichtigt wird, muss auf die Teilstichprobe der beiden Kurse $\mathrm{K}_{4}$ und $\mathrm{T}_{5}$ verzichtet werden, da die Lehrervariable an dieser Schule nicht konstant gehalten werden konnte.

Die Hausaufgabendauer $\left(0=0-5 \mathrm{~min}, 1=5^{-10} \mathrm{~min}, 2=10-15 \mathrm{~min}, 3=15-30 \mathrm{~min}\right)$ unterscheidet sich nicht signifikant zwischen den Kursen und Gruppen. Rechnet man die Angabe jedes Schülers näherungsweise in Minuten um $(o=2.5 \mathrm{~min}, 1=7.5 \mathrm{~min}, 2=12.5 \mathrm{~min}$, $3=22.5 \mathrm{~min}$ ), ergibt sich für die Kontrollgruppe ein mittlerer Wert für die Hausaufgabendauer von $M_{\text {trad }}=8.45 \mathrm{~min}, S D=5.55 \mathrm{~min}$, und für die Treatmentgruppe von $M_{\text {flip }}=10.10 \mathrm{~min}$, $S D=5.85$ min, zum Prezeitpunkt. Der Gesamtmittelwert der Hausaufgabendauer aller Schüler zum Prezeitpunkt liegt bei $M_{\text {total }}=9.18 \mathrm{~min}, S D=5.74 \mathrm{~min}$.

In den Variablen Begeisterung für digitale Geräte und Selbstkonzept digitale Kompetenz gibt es ebenfalls keine Gruppenunterschiede. Von Interesse sind diese beiden Variablen, wenn der Einfluss des Geschlechts untersucht wird. 
Zusammenfassend ist die Stichprobe kurs- und schulübergreifend homogen hinsichtlich der zentralen abhängigen Variablen Leistung, Motivation, Fachinteresse und Selbstkonzept. Welcher Lehrer in diesen Kursen unterrichtet, hat keinen signifikanten Einfluss auf diese Variablen. Allerdings ist ein signifikanter Notenunterschied im Fach Physik zwischen Kontroll- und Treatmentgruppe zu verzeichnen. Forschungshypothese Hza behauptet, dass das Leistungsniveau im Fach Physik die Wirkung des Flipped Classroom nicht beeinflusst. Hier wird zu untersuchen sein, ob die leistungsbezogene Lernausgangslage, die in Kontroll- und Treatmentgruppe unterschiedlich ist, eine Rolle spielt. Die Lehrerunterstützung und die Hausaufgabendiszplin sind stark kursabhängig. Hier gibt es signifikante Unterschiede sowohl zwischen den Kursen als auch zwischen Kontroll- und Treatmentgruppe. Die Hausaufgabendauer hingegen unterscheidet sich gruppen- und kursübergreifend nicht.

Nach der statistischen Beschreibung der Lerngruppe und der Lernausgangslage beider Gruppen sollen nun Antworten auf die drei Forschungsfragen gefunden werden, die in Kap. 3 formuliert und in insgesamt sechs Hypothesen übersetzt wurden.

\subsection{Forschungsfrage 1: Einfluss auf Lernleistung, Motivation, Interesse und Selbstkonzept}

Die erste Forschungsfrage richtet sich darauf, inwiefern der Flipped Classroom im Vergleich zum traditionellen Physikunterricht die Lernleistung und die affektiven Lernmerkmale beeinflusst. Hier geht zunächst nur die Methode des Unterrichts (traditionell vs. flipped) als nominaler Faktor in die ANOVA ein. Erst die Forschungsfrage 2 untersucht moderierende Einflüsse, also mögliche Interaktionen anderer unabhängiger Faktoren mit der Methode.

\subsubsection{Hypothese 1a: Lernleistung}

H1a. Der Flipped Classroom steigert die Lernleistung im Vergleich zu traditionellem Physikunterricht.

Der Levene-Test für die Lernleistung, dargestellt im normierten Lernzuwachs Hakes $g$ (vgl. 5.5), fällt nicht signifikant aus ( $p=.954)$, Varianzhomogenität kann angenommen werden, ebenso Normalverteilung (Shapiro-Wilk: $d f_{\text {trad }}=79, p=.777, d f_{\text {flip }}=70, p=.711$ ). Tab. 11 und Abb. 17 zeigen die Werte der mittleren normierten Gain Scores $g$ von Kontroll- und Treatmentgruppe und das Ergebnis der ANOVA.

Es wird deutlich, dass die Lernleistung der Schüler, die im Flipped Classroom unterrichtet wurden, signifikant höher ist als die traditionell unterrichteten Schüler, $F(1,147)=9.801$, $p=.002$. Die Leistungswerte liegen in der Kontrollgruppe im Intervall $.00 \leq \mathrm{g} \leq .86$ und in der Treatmentgruppe im Intervall $.09 \leq g \leq .89$. Die Stärke des Effekts kann in der einfaktoriellen ANOVA als partielles Eta-Quadrat berechnet werden, welches den Anteil der aufgeklärten 
Tab. 11. Vergleich des Lernzuwachses (Hakes g) von Kontroll- und Treatmentgruppe

\begin{tabular}{|c|c|c|c|c|c|c|c|c|c|}
\hline & \multicolumn{3}{|c|}{ traditionell } & \multicolumn{3}{|c|}{ flipped } & \multirow[b]{2}{*}{$F$} & \multirow[b]{2}{*}{$p$} & \multirow[b]{2}{*}{$\eta_{p^{2}}$} \\
\hline & $N$ & $M$ & $S D$ & $N$ & $M$ & $S D$ & & & \\
\hline Lernzuwachs g (0-1) & 79 & .37 & .18 & 70 & .46 & .17 & 9.801 & .002 & .063 \\
\hline
\end{tabular}
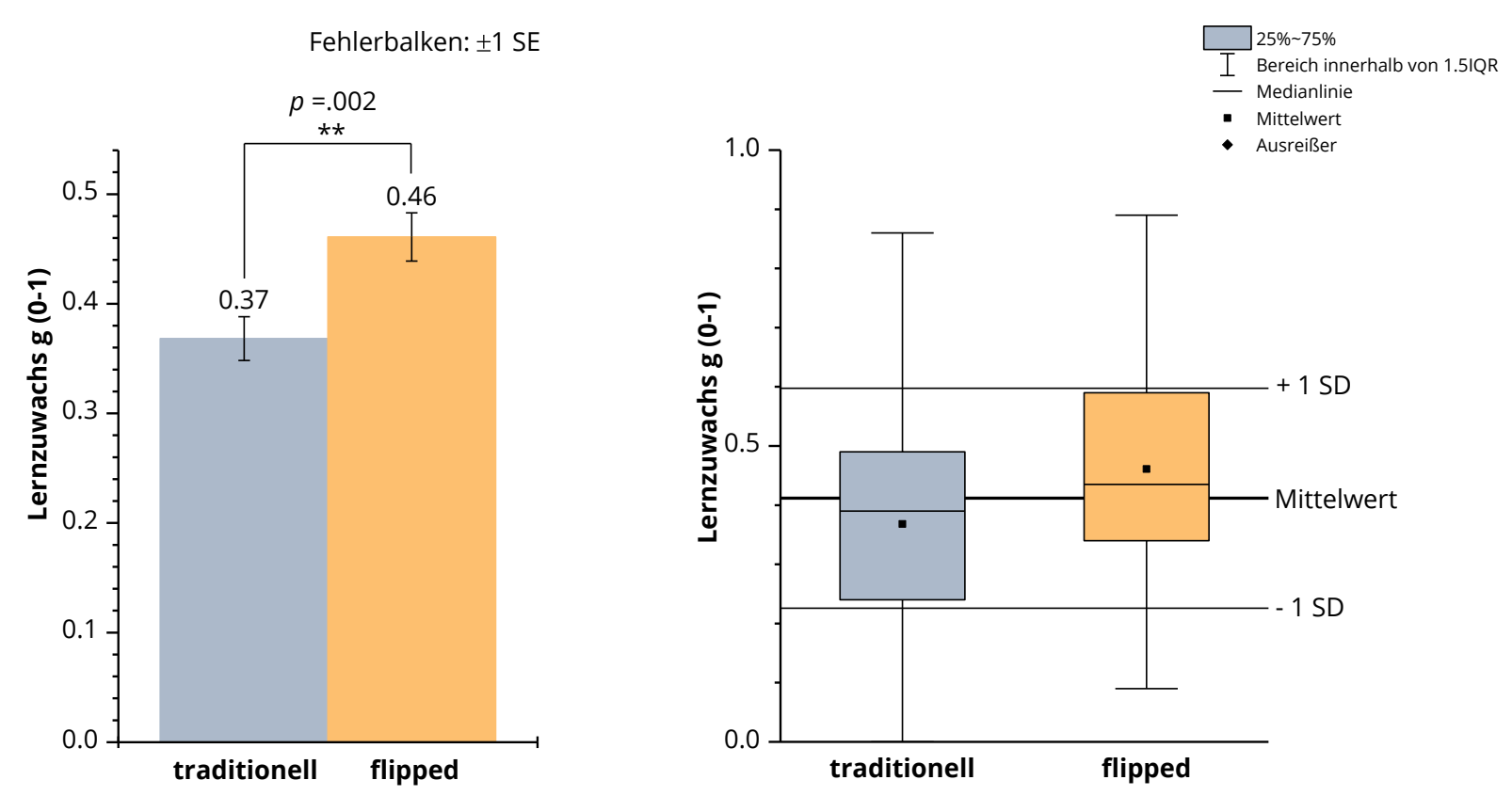

Abb. 17. Mittlere Lernzuwächse beider Gruppen (Hakes g) und Boxplots

Varianz an der Gesamtvarianz angibt. Mit $\eta_{p}{ }^{2}=.063$ bedeutet das, dass etwa 6\% der Gesamtvarianz der Variable $g$ durch die Unterrichtsmethode aufgeklärt wird. Nach Cohen (1988) entspricht das einem mittleren Effekt.

Die Basisform des Flipped Classroom, die in dieser Studie implementiert wurde, sorgte im Physikunterricht demnach für einen höheren Lernzuwachs im Vergleich zum traditionellen Physikunterricht. Die Schüler im Flipped Classroom hatten bessere Voraussetzungen, um die Aufgaben des Fachwissenstests zu bearbeiten, und demnach eine bessere Lernleistung. Die Forschungshypothese Hia wurde bestätigt.

\subsubsection{Hypothese 1b: Motivation, Fachinteresse und Selbstkonzept}

H1b. Der Flipped Classroom hat einen positiven Effekt auf die Entwicklung der affektiven Lernmerkmale Motivation, Fachinteresse und Selbstkonzept im Vergleich zum traditionellen Physikunterricht.

Hypothese Hıb behauptet die Verbesserung der affektiven Lernmerkmale der Schüler durch den Flipped Classroom. Im traditionellen Unterricht ist keine Veränderung dieser Merkmale zu erwarten, da die Schüler der Kontrollgruppe methodisch nicht anders als sonst 
Tab. 12. Korrelationsmatrix der Gain Scores der affektiven Lernmerkmale

\begin{tabular}{|c|c|c|c|}
\hline & $\Delta$ Motivation & $\Delta$ Fachinteresse & $\Delta$ Selbstkonzept \\
\hline$\Delta$ Motivation & & $\begin{array}{c}r=.18 \\
p=.028\end{array}$ & $\begin{aligned} r & =.26 \\
p & =.001\end{aligned}$ \\
\hline$\Delta$ Fachinteresse & $\begin{array}{c}r=.18 \\
p=.028\end{array}$ & & $\begin{aligned} r & =.26 \\
p & =.001\end{aligned}$ \\
\hline$\Delta$ Selbstkonzept & $\begin{aligned} r & =.26 \\
p & =.001\end{aligned}$ & $\begin{aligned} r & =.26 \\
p & =.001\end{aligned}$ & \\
\hline
\end{tabular}

unterrichtet wurden. Die Änderungen der affektiven Merkmale Motivation, Fachinteresse und Selbstkonzept zwischen Pre- und Postmessung werden als Differenz-Gain Scores $\Delta$ gemessen.

Der Levene-Test fällt für alle drei Variablen nicht signifikant aus, allerdings sind die Gain Scores nicht normalverteilt (Shapiro-Wilk: $p_{\Delta M o t}<.001, p_{\Delta F a c h}<.001, p_{\Delta S e l b s t}<.001$ ). Demnach sind die Voraussetzungen für eine Varianzanalyse nicht erfüllt. Doch aufgrund der Größe der Stichprobe $(\mathrm{N}=150)$ wird hier die Verletzung der Normalverteilung toleriert. Alle drei Gain Scores korrelieren signifikant miteinander (vgl. Tab. 12). Die inhaltlich begründete Interdependenz zwischen den drei affektiven Variablen (vgl. Kap. 2.2) zeigt sich demnach auch in den Korrelationen.

Eine MANOVA, in der die Varianzen mehrerer miteinander korrelierender und abhängiger Variablen als Gruppe analysiert werden, zeigt unter Verwendung der Pillai-Spur, dass die Methode einen signifikanten Einfluss auf diese Variablengruppe hat, $V=.058$, $F(3,146)=2.986, p=.033$. Die Methode Flipped Classroom trägt also zu einer signifikanten Verbesserung der affektiven Lernmerkmale bei. Der Effekt liegt mit $\eta_{p}{ }^{2}=.058 \mathrm{im}$ kleinen bis mittleren Bereich. Nachfolgend sollen die Variablen in univariaten ANOVAs einzeln dargestellt werden.

Die intrinsische Motivation, sich mit Physik zu beschäftigen, nimmt in der Kontrollgruppe innerhalb der drei Monate, in denen sie traditionell unterrichtet wird, deutlich ab (vgl. Tab. 13). Betrachtet man lediglich die Schüler der Kontrollgruppe, so ist der Rückgang der Motivation signifikant, $F(1,79)=14.573, p<.001, \eta_{p}{ }^{2}=.156$ (vgl. Abb. 18). Diese drastische Abnahme der Motivation in der Kontrollgruppe ist zu diskutieren. Der mittlere Wert der Motivation in der Treatmentgruppe liegt zum Prezeitpunkt deutlich, aber nicht signifikant unterhalb des Werts der Kontrollgruppe (vgl. Abb. 18). Doch im Gegensatz zur Kontrollgruppe bleibt dieser Wert in der Treatmentgruppe sehr stabil.

Betrachtet man die Entwicklung der Variable Motivation von Kontroll- und Treatmentgruppe zwischen Pre- und Postzeitpunkt im direkten Vergleich, so ist der Unterschied in der Entwicklung signifikant, $F(1,148)=6.413, p=.012, \eta_{p}{ }^{2}=.042$. Nach Cohen (1988) entspricht das einem kleinen Effekt. Während die Motivation der Schüler im Flipped Classroom auf konstantem Niveau bleibt, fällt sie bei den Schülern im traditionellen Unterricht massiv ab. Der Flipped Classroom hat in diesem Sinne einen Einfluss auf die Motivation, insofern als sie 
Tab. 13. Vergleich der Entwicklungen in den affektiven Lernmerkmalen in Einzel-ANOVAs

\begin{tabular}{|c|c|c|c|c|c|c|c|c|c|}
\hline & \multicolumn{3}{|c|}{ traditionell } & \multicolumn{3}{|c|}{ flipped } & \multirow[b]{2}{*}{$F$} & \multirow[b]{2}{*}{$p$} & \multirow[b]{2}{*}{$\eta p^{2}$} \\
\hline & $N$ & $M$ & $S D$ & $N$ & $M$ & $S D$ & & & \\
\hline$\Delta$ Motivation $(-3 \ldots+3)$ & 80 & -.18 & .42 & 70 & -.01 & .41 & 6.413 & .012 & .042 \\
\hline$\Delta$ Fachinteresse $(-3 \ldots+3)$ & 80 & -.20 & .60 & 70 & -.09 & .63 & 1.282 & .259 & .009 \\
\hline$\Delta$ Selbstkonzept $(-3 \ldots+3)$ & 80 & -.02 & .38 & 70 & .11 & .35 & 4.565 & .034 & .030 \\
\hline
\end{tabular}

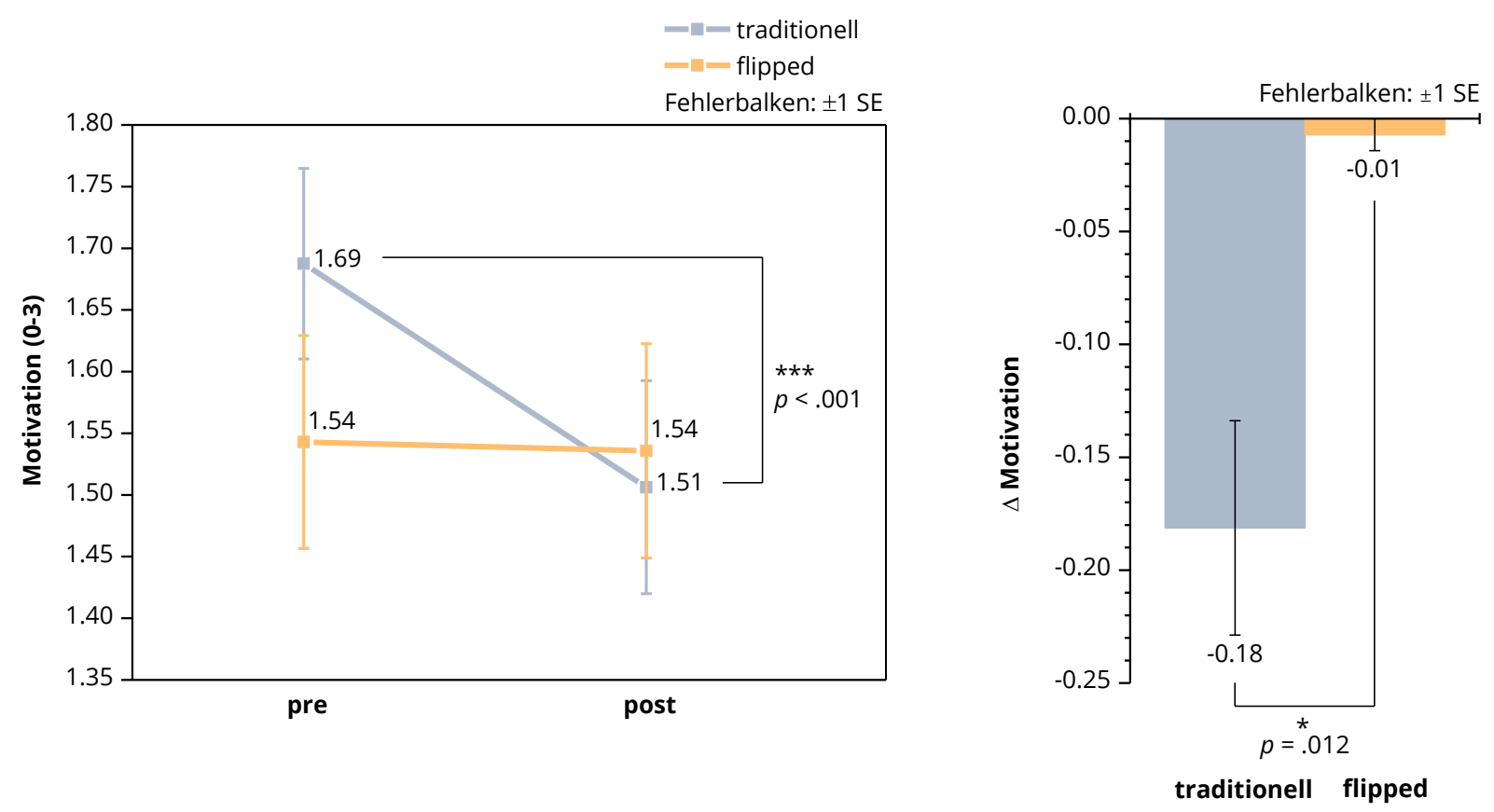

Abb. 18. Entwicklung der Motivation

nicht wie in der Kontrollgruppe geringer wird. Damit entsprechen die Ergebnisse der in Hib ausgedrückten Erwartung, dass der Flipped Classroom einen positiven Effekt auf die Entwicklung der Motivation hat. Der Flipped Classroom vermag eine negative Entwicklung aufzuhalten, deren Ursache zu diskutieren ist.

Das Fachinteresse nimmt in der Kontrollgruppe signifikant ab, $F(1,79)=8.778, p=.004$, $\eta_{p}{ }^{2}=.100$ (vgl. Abb. 19). In der Treatmentgruppe zeigt sich ebenfalls eine Interessensabnahme, die jedoch auf dem Niveau von $\alpha=.05$ nicht signifikant ist, $F(1,69)=1.291, p=.260$. Werden die Rückgänge im Fachinteresse beider Gruppen miteinander verglichen, gibt es keinen signifikanten Unterschied zwischen Kontroll- und Treatmentgruppe, $F(1,148)=1.282, p=.259$. Der Flipped Classroom wirkte sich demnach nicht anders auf die Entwicklung des Fachinteresses aus als der traditionelle Unterricht. Betrachtet man die Stichprobe als Ganzes, ist der Mittelwert von $\Delta$ Fachinteresse (= post - pre) signifikant von Null verschieden, $F(1,149)=8.468$, 

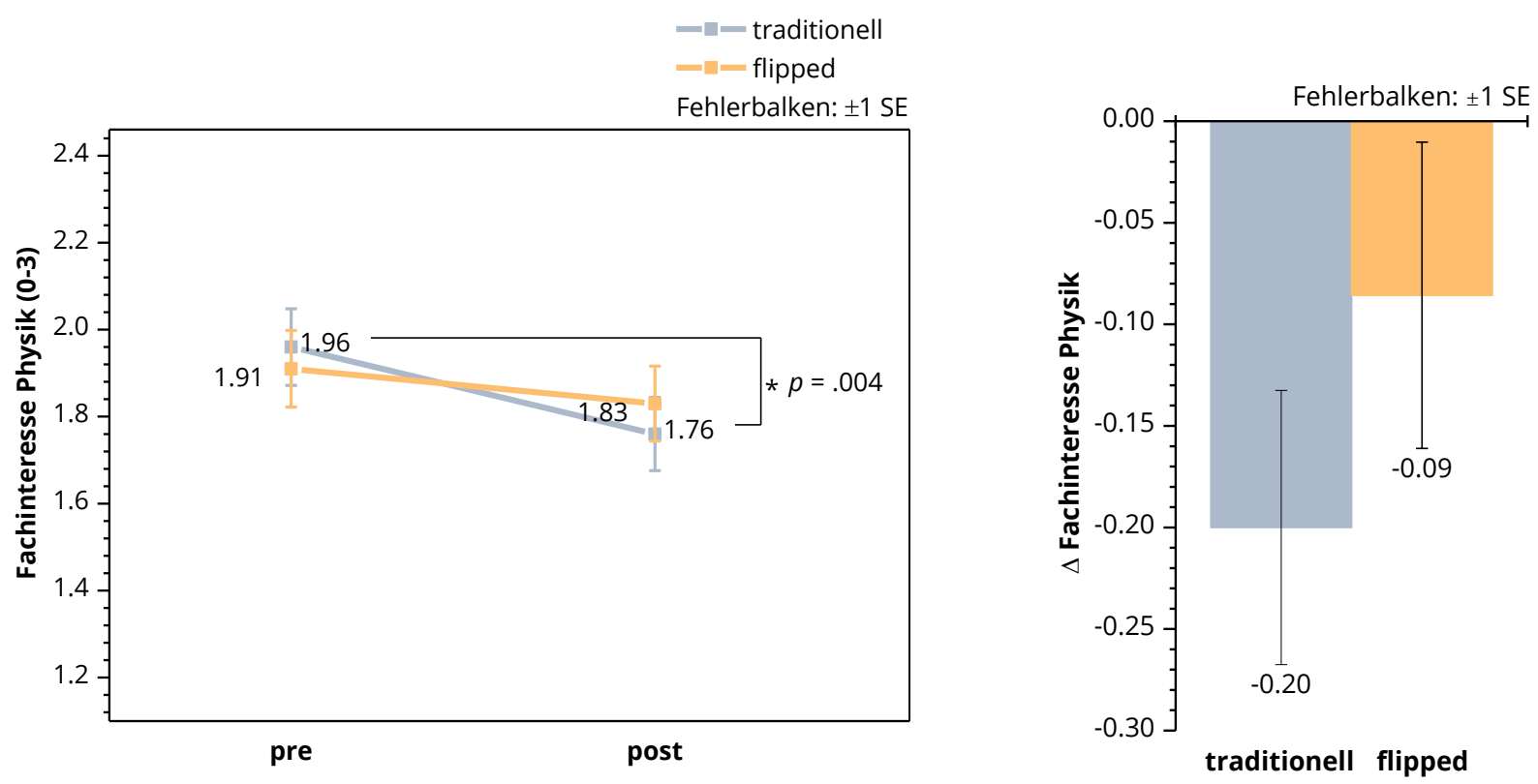

Abb. 19. Entwicklung des Fachinteresses im Fach Physik
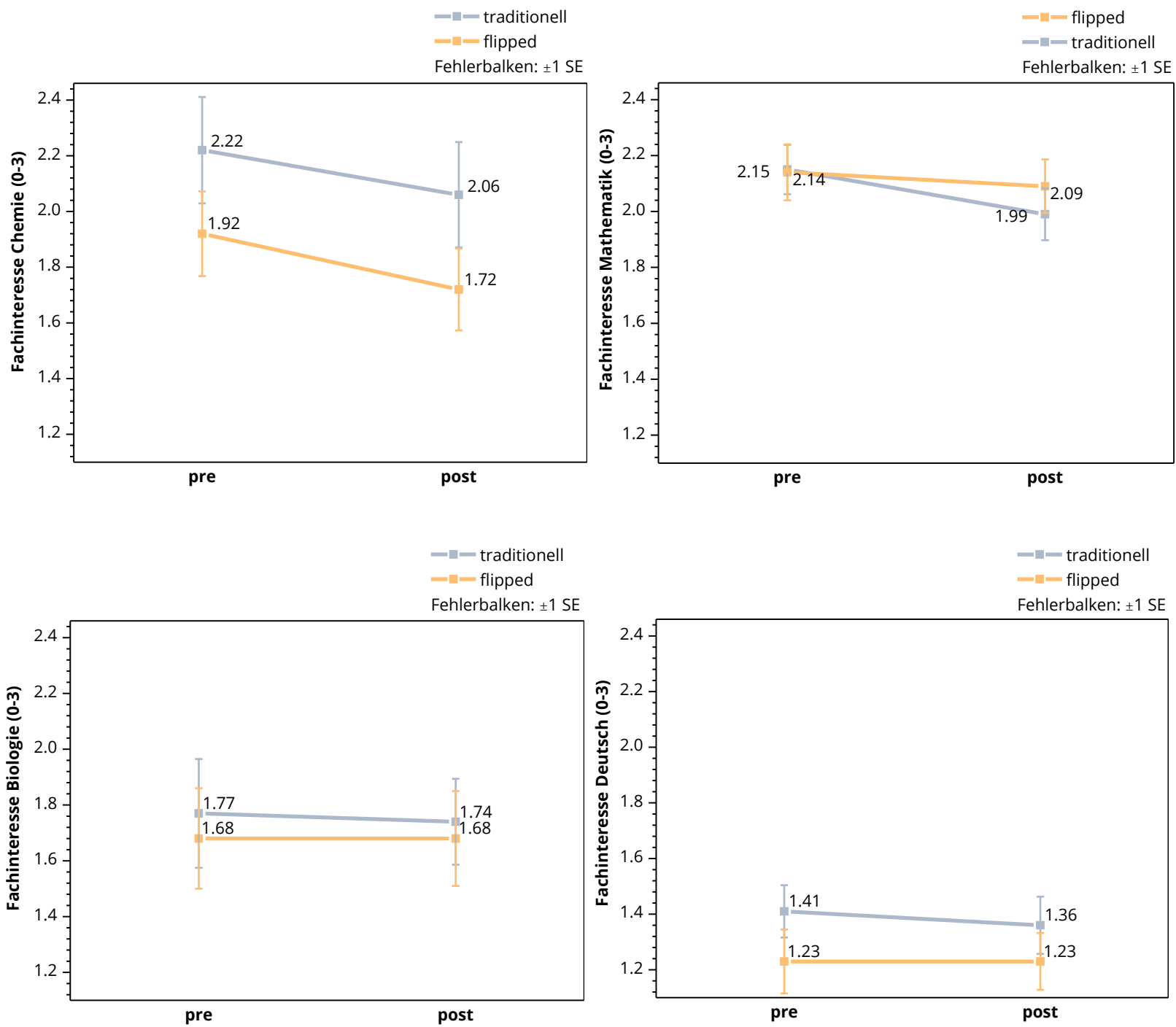

Abb. 2o. Entwicklung des Fachinteresses in den Fächern Mathematik, Chemie, Biologie und Deutsch 
$p=.004$. Unabhängig von der Unterrichtsmethode, in der die Schüler unterrichtet wurden, sinkt das Interesse am Unterrichtsfach Physik.

Der Flipped Classroom mag diese Entwicklung offenbar auch mesoskopisch nicht aufzuhalten. In den schriftlichen Rückmeldungen zum Flipped Classroom wird kritisch zu prüfen sein, ob es Gründe gibt, warum die Schüler auch im Flipped Classroom das Interesse am Unterrichtsfach verlieren. Grundsätzlich stehen die Ergebnisse in Einklang mit anderen Studien, die den Fachinteressensrückgang innerhalb der Schulbiographie von Schülern beobachtet haben (Daniels 2008; Hannover \& Kessels 2001; Hidi 2000; Hoffmann et al. 1998; Kleickmann 2011; Krapp 2002; Wild \& Hofer 2000). Bezüglich des Fachinteresses trifft H1b nicht zu.

Um einen Kontext für die Entwicklung des Interesses im Fach Physik zu geben, werden in Abb. 20 noch die Diagramme zum Interesse an den anderen Unterrichtsfächern dargestellt. Es zeigt sich, dass das Fach Physik keine Ausnahme bildet, sondern Teil einer breiteren Interessensdegeneration ist. In den Fächern Biologie $(N=56)$ und Deutsch $(N=150)$, das in beiden Gruppen ohnehin den niedrigsten Interessenswert hatte, ist dieser Rückgang nicht messbar, aber in den Fächern Mathematik und Chemie ist die gleiche Tendenz zu beobachten wie im Fach Physik. Im Dreimonatszeitraum Februar/März bis April/Mai ist auch dort in der gesamten Stichprobe ein Interessensabfall zu verzeichnen, der im Fach Mathematik signifikant $(N=148, p=.043)$ ist und im Fach Chemie $(N=43, p=.088)$ knapp oberhalb der Signifikanzgrenze von $\alpha=.05$ liegt.

Das physikbezogene Selbstkonzept entwickelt sich im Flipped Classroom besser als im traditionellen Unterricht (Abb. 21). Während es in der Kontrollgruppe leicht rückläufig ist, verbessert es sich in der Treatmentgruppe. Der Unterschied in den Änderungen des Selbstkonzepts zwischen Kontroll- und Treatmentgruppe ist signifikant, $F(1,148)=4.565, p=.034$. Die Effektgröße $\eta_{p}^{2}=.030$ entspricht einem kleinen Effekt. Die Hypothese H1b kann im Hinblick auf das physikbezogene Selbstkonzept angenommen werden.
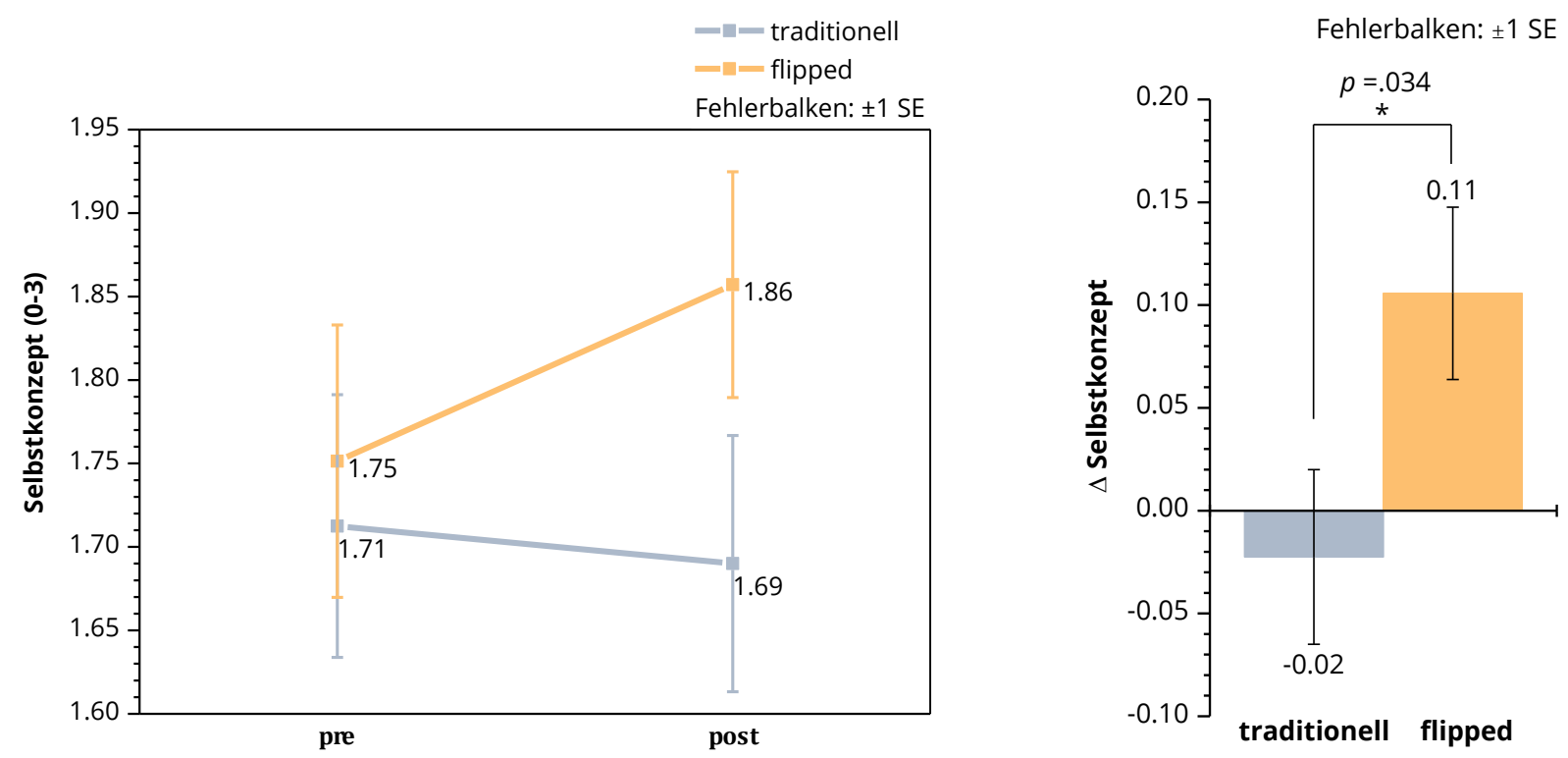

Abb. 21. Entwicklung des physikbezogenen Selbstkonzepts 
Die Selbstnote Physik, mit der die Schüler ihre Leistungen im Fach Physik innerhalb der letzten zwei Monate in Notenpunkten (o-15) einschätzen sollten, ist mit dem Selbstkonzept verwandt und wurde zu beiden Messzeitpunkten erhoben. So lässt sich ein Differenz-Gain Score $\Delta$ bilden, der die Entwicklung der notenmäßigen Selbsteinschätzung der Schüler abbildet. Es zeigt sich, dass diese Selbsteinschätzung im Laufe der Unterrichtsreihe in beiden Gruppen schlechter wurde, $\Delta_{\text {trad }}=-.61, S D=2.22 ; \Delta_{f l i p}=-.37, S D=2.05$. Die Schüler der Kontrollgruppe schätzten ihre Leistungen im Mittel mehr als einen halben Notenpunkt schlechter ein als noch vor der Unterrichtsreihe. Für sich betrachtet ist dieser Notenabfall in der Kontrollgruppe signifikant, $F(1,79)=6.064, p=.016, \eta_{p}{ }^{2}=.071$. Auch die Schüler der Treatmentgruppe schätzten ihre Noten im Mittel schlechter ein als zuvor, jedoch ist der Notenabfall hier nicht signifikant, $F(1,69)=2.295, p=.134$. Vergleicht man die Entwicklung der mittleren Notenselbsteinschätzung beider Gruppen miteinander, ist der Unterschied zwischen den Gruppen nicht signifikant, $F(1,148)=.471, p=.493$.

Zusammenfassend lässt sich sagen, dass sich im Flipped Classroom Lernleistung, Motivation und Selbstkonzept positiv im Vergleich zum traditionellen Unterricht entwickelt haben. Die Lernleistung und das Selbstkonzept stiegen im Flipped Classroom signifikant stärker an als in der Kontrollgruppe. Der Zusammenhang zwischen der kognitiven Leistung und den affektiven Lernmerkmalen steht damit im Einklang zum Stand bisheriger Forschung (Baumeister et al. 2003; Marsh et al. 2005; Schiefele \& Schreyer 1994; Valentine et al. 2004; Wylie 1979). Das Fachinteresse, welches nach Häussler \& Hoffmann (2000) stark, aber nicht ausschließlich durch das Selbstkonzept beeinflusst wird, entwickelt sich in dieser Studie nicht synchron zum Selbstkonzept.

\subsubsection{Diskussion}

Die Ergebnisse zeigen, dass die Schüler im Flipped Classroom bessere kognitive Leistungen erzielten als ihre Mitschüler im traditionellen Unterricht. Damit bestätigt die Studie, dass Flipped Classroom durch die höhere kognitive Aktivierung auch im schulischen Physikunterricht der Oberstufe einen günstigen Einfluss auf die kognitive Lernentwicklung besitzt. Bisherige Studien zum Flipped Classroom wurden hauptsächlich in englischsprachigen Bildungssystemen durchgeführt und nahmen dabei kaum die Physikausbildung in den Blick. Die vorliegende Studie zeigt, dass die Lernleistung durch den Flipped Classroom im experimentorientierten Physikunterricht des deutschsprachigen Schulsystems größer ist als im traditionellen Unterricht. Sie erweitert damit das positive Bild, das Studien in anderen Disziplinen, Ländern und Bildungssystemen von dieser Unterrichtsmethode gezeichnet haben (Pearson Education 2013; Aş1ksoy \& Özdaml 2016; Bhagat et al. 2016; Bidwell 2014; Casasola et al. 2017; Cieliebak 2014; Clark 2015; Day \& Foley 2006; Deslauriers et al. 2011; Fautch 2015; Foldnes 2016; Gilboy et al. 2015; Goerres et al. 2015; González-Gómez et al. 2016; Gregorius 2017; Lin \& Chen 2016; Love et al. 2014; MacKinnon 2015; Marcey \& Brint 2012; McLaughlin et al. 2014; Missildine et al. 2013; Pierce \& Fox 2012; Sadaghiani 2012; Schultz et al. 2014; Şengel 2016; Sun \& Wu 2016; Van Sickle 2016; Webster et al. 2016; Wong \& Chu 2014; Zoe et al. 2014). Die Vielzahl der 
Arten, wie Flipped Classroom im Unterricht eingeführt und umgesetzt werden kann, erklärt die Bandbreite an Ergebnissen, die die Studien auf diesem Gebiet zu Tage förderten. So gibt es auch viele Studien, in denen es keine signifikanten Unterschiede in den Lernleistungen im Flipped Classroom und traditionellen Lehrformaten gab (Bell 2015; Bishop 2013; FindlayThompson \& Mombourquette 2014; Jensen et al. 2015; Johnson \& Renner 2012; Lape et al. 2014; Marlowe 2012; Munson \& Pierce 2015; Zheng et al. 2014). Ausgehend von der vorliegenden Studie lässt sich sagen, dass Flipped Classroom im Physikunterricht eine schüleraktivierende Methode ist, in der positive Lernergebnisse zu erwarten sind, wenn die hier implementierte Basisform (vgl. Kap. 2.3.2) verwendet wird und wenn als Lernziel der Zuwachs an deklarativem Wissen steht. Wenn jedoch eine umfassende physikalische Kompetenz erworben werden soll, die neben dem Fachwissen auch erkenntnisgewinnende, kommunikative und beurteilende Kompetenzen einschließt, muss jedoch überlegt werden, ob der Flipped Classroom auch hier einen Beitrag leisten kann.

Die affektiven Lernerfolge müssen differenziert betrachtet werden. Vor allem muss berücksichtigt werden, dass diese Studie im Oberstufenunterricht stattfand. Das bedeutet, dass sämtliche Schüler die Wahl getroffen hatten, das Fach Physik in der Oberstufe weiterhin zu belegen. Die Einstellung gegenüber dem Fach Physik dürfte damit im Mittel besser sein als in der Mittelstufe. In der vorliegenden Studie verbesserte sich das physikbezogene Selbstkonzept der Schüler deutlich und ergänzt die Befunde von Aşıksoy \& Özdamlı (2016), Enfield (2013) und Findlay-Thompson \& Mombourquette (2014) für den schulischen Physikunterricht. Der Zusammenhang von Selbstkonzept und Lernleistung, der generell sehr eng ist (Baumeister et al. 2003; Marsh \& Craven 2006; Valentine et al. 2004; Wylie 1979), zeigte sich auch hier. Bezeichnend ist, dass das Selbstkonzept der Schüler im Flipped Classroom sich positiv entwickelt hat, obwohl in der Zeit des Treatments keine schulischen Leistungstests stattfanden, deren Benotung einen Einfluss auf das Selbstkonzept hätte haben können. Durch das Fehlen sachlicher Bezugsnormen liegt auf der Hand, dass es allein soziale und individuelle Bezugsnormen waren, an denen die Schüler ihr Selbstkonzept entwickeln konnten (vgl. Kap. 2.2.3). Im Flipped Classroom schätzten die Schüler ihre physikbezogenen kognitiven Fähigkeiten höher ein als die Schüler des traditionellen Unterrichts. Die positiven Erfahrungen in den Clicker-Quizze und dem kooperativen Bearbeiten und Lösen von Aufgaben führten demnach zu einem höheren Selbstkonzept als bei den Schülern der Kontrollgruppe. Die Entwicklungen der Lernleistung und des Selbstkonzepts in der Studie entsprechen den in den Hypothesen gestellten Erwartungen. Beide Variablen zeigen eine im Vergleich zur Kontrollgruppe deutlich positive Entwicklung.

Es ist schwierig, über das themenspezifische Selbstkonzept Induktion eine Aussage zu treffen. Da im Fachwissenstest zum Prezeitpunkt einige Schüler bereits hohe Punktzahlen erzielten, steht zu vermuten, dass in der Stichprobe zum Teil ein ausgeprägtes Selbstkonzept Induktion zum Prezeitpunkt vorgelegen haben muss, das hätte gemessen werden können. Im Nachhinein wäre die Pre-Messung des Selbstkonzepts Induktion deshalb gerechtfertigt gewesen und hätte Rückschlüsse über mögliche Veränderungen während der Unterrichtsreihe er- 
laubt. Für sich genommen zeigen die Postwerte des Selbstkonzepts Induktion keine Unterschiede, doch ist denkbar, dass die Ausgangslage in den Gruppen tatsächlich unterschiedlich war.

Die Variable Selbstnote Physik bot Raum für Missverständnisse. Das Item »Wie viele Punkte würden Sie sich im Fach Physik für den Zeitraum der letzten 2 Monate selbst geben?« bezieht sich auf die selbsteingeschätzten Fähigkeiten der Schüler und damit direkt auf ihr Selbstkonzept. Doch lässt sich die Frage auch auf die Demonstration ihrer Fähigkeiten beziehen. In einem offenen und kollaborativen Unterricht sind erfahrungsgemäß weniger Möglichkeiten gegeben, fähigkeitsdemonstrierende mündliche Unterrichtsbeiträge zu liefern, die zu einer guten Note führen, als das in einem traditionellen fragend-entwickelnden Unterricht der Fall ist. Das Item ist deshalb unscharf und kann verstanden werden als »Wie viele Punkte würden Sie sich für ihr Können im Fach Physik für die letzten 2 Monate geben? « oder als »Wie viele Punkte würden Sie sich geben für die Leistung, die Sie im Fach Physik gezeigt haben?«. Aufgrund der Offenheit des Items lässt sich nicht entscheiden, in welchem Sinne die Schüler die Frage verstanden haben, so dass kein großer Erkenntnisgewinn aus diesem Item hervorgeht. Eine Folgeuntersuchung müsste hier präziser formulieren.

Die intrinsische Motivation, sich mit Physik zu beschäftigen, blieb bei den Schülern im Flipped Classroom auf einem konstanten Wert, während sie im traditionellen Unterricht deutlich absank. Der Unterschied in der Motivationsentwicklung zwischen Kontroll- und Treatmentgruppe ist signifikant. Nur im Vergleich zum drastischen Motivationsverlust in der Kontrollgruppe lässt sich also die Hypothese halten, dass der Flipped Classroom einen positiven Effekt auf die Entwicklung der Motivation besitzt. In der vorliegenden Studie trug der Flipped Classroom nicht dazu bei, dass sich die Motivation der Schüler in irgendeiner Form verbesserte. Woran könnte das - trotz stärkerer kognitiver Aktivierung und trotz besserer Möglichkeit zur Erfahrung von Kompetenz, Selbstbestimmung und sozialer Bezogenheit (vgl. Kap. 2.2.1) - gelegen haben?

Wie bereits erwähnt belegen Schüler das Fach Physik in der Oberstufe in der Regel nur, wenn sie bereits über eine höhere mittlere intrinsische Motivation bezüglich der Inhalte und des Faches besitzen. Unbenommen ist dabei die Tatsache, dass auch extrinsische Motivationen eine Rolle spielen, etwa Erwartungen über die unterrichtenden Lehrer, die Noten im Aufwand-Ergebnis-Verhältnis oder der Mangel an Motivation und Fähigkeiten für andere Naturwissenschaften. Wenn die Motivation von vornherein auf einem höheren Wert liegt, als gemeinhin zu erwarten ist, ist es möglicherweise schwieriger, diese durch Unterrichtsmethoden noch zu steigern. Für diese Überlegung spricht, dass nach Krapp (1992) bei besonders hoch entwickeltem individuellem Interesse die Auslösebedingungen für situatives Interesse von untergeordneter Bedeutung oder gar irrelevant sind. Das bedeutet, dass der Flipped Classroom bei Schülern, die bereits eine hohe intrinsische Motivation für die Inhalte des Fachs Physik besaßen, kein neues aufkommendes oder situationales Interesse fördern konnte, welches sich in zusätzlicher Motivation äußert (vgl. Kap. 2.2.2). 
Jedoch musste festgestellt werden, dass der traditionelle Unterricht in der Kontrollgruppe dazu führte, dass die mittlere Motivation dort deutlich absank. Der traditionelle Physikunterricht hatte also einen negativen Effekt auf die Motivation. Offensichtlich waren die motivationalen Anreize für Schüler zu niedrig, um die Motivation auch nur auf dem Niveau des Pretestzeitpunkts zu halten. Die Skala zur Messung der intrinsischen (Lern-)Motivation, die der PISA-Hauptstudie entnommen ist, deckt zum großen Teil auch das individuelle Interesse ab, da beide eng miteinander verknüpft sind und das Interesse die inhaltliche Bedingung der Motivation ist. Der massive Rückgang an intrinsischer Motivation geht mit einem Verlust an individuellem Interesse einher. Gemäß der vorliegenden Studie verloren die Schüler im traditionellen Physikunterricht der Oberstufe drastisch intrinsische Motivation und Interesse an physikalischen Themen - und das, obwohl sie freiwillig Physik als Fach in der 11. Jahrgangsstufe weiterführten. Zu einer Aufklärung dieser negativen Interessensentwicklung in der Oberstufe kann diese Studie keinen Beitrag leisten. Denkbar ist, dass dieser Motivationsrückgang mit den Inhalten der Unterrichtsreihe »Induktion und elektromagnetischer Schwingkreis « zusammenhängt, die für die Studie gewählt wurde. Generell könnten auch enttäuschte Erwartungen an die Inhalte der Oberstufenphysik eine Rolle spielen, die sich in den drei Monaten zwischen Pre- und Posttest im zweiten Schulhalbjahr manifestieren. Festzuhalten ist lediglich, dass der Flipped Classroom diesen Motivationsrückgang - zumindest innerhalb des dreimonatigen Zeitraums der Studie - aufzuhalten vermochte.

Die Entwicklung der Motivation und des Sachinteresses spiegelt sich in der Entwicklung des Fachinteresses Physik, welches besonders in der Kontrollgruppe nachließ. Das Sachund Fachinteresse stehen in einem engen Zusammenhang, lassen sich aber nicht aufeinander reduzieren (vgl. Kap. 2.2.2). In dieser Studie heißt das: Bei den Schülern im traditionellen Physikunterricht nahmen die Werte für die intrinsische Motivation und das Fachinteresse zwischen Pre- und Postzeitpunkt signifikant ab. Hier bestätigt sich auch für Oberstufenkurse im Fach Physik, was generell über die Entwicklung schulischer Fachinteressen festgestellt wird: Das Interesse an Schulfächern sinkt im Laufe der Schulzeit kontinuierlich ab, besonders in den MINT-Fächern (Hoffmann et al. 1998; Krapp 2002) und auch schon auf Zeitskalen von Monaten (Daniels 2008) und Wochen (Weber 2003; Winkelmann 2013).

Bei den Schülern im Flipped Classroom gab es hingegen in der Motivation und im Fachinteresse keine signifikanten Veränderungen zwischen Pre- und Postzeitpunkt, auch wenn das Fachinteresse tendenziell rückläufig war. Vergleicht man die Entwicklung von Kontroll- und Treatmentgruppe miteinander, ist der Rückgang des Fachinteresses in der Kontrollgruppe nicht mehr signifikant. Das bedeutet, im direkten Vergleich miteinander gibt keinen deutlich messbaren Unterschied in der Fachinteressensentwicklung in beiden Gruppen, nur relativ zum eigenen mittleren Prewert verschlechterte sich das Fachinteresse in der Kontrollgruppe.

Das Fachinteresse Physik speist sich aus mehreren Quellen: dem individuellen Sachinteresse, dem unmittelbaren situationalen Interesse, welches durch didaktisch interessant aufbereiteten Unterricht gefördert wird, und dem sozialen Lernklima in der Physikklasse (vgl. Kap. 2.2.2). Unterrichtliche Veränderungen sollten sich demnach am ehesten in der Variable Fachinteresse auswirken, wenn sie interessensrelevante Bedeutung besitzen. Das Experiment 
im Physikunterricht besitzt eine solche interessensrelevante Bedeutung. Zwar stand sowohl in der Kontroll- als auch in der Treatmentgruppe das Experiment im Zentrum des Unterrichts, doch war die mediale Präsentation der Experimente für die Schüler fundamental verschieden. In der Kontrollgruppe wurden die Schüler unmittelbare Zeugen von Demonstrationsexperimenten, die ihnen greifbar vor Augen standen, während die Schüler der Treatmentgruppe lediglich videografierte Demonstrationsexperimente in knapper Form sahen. Wenn es jedoch zwischen Kontroll- und Treatmentgruppe keine messbaren Unterschiede in der Veränderung des Fachinteresses Physik während der Studie gab, ist auszuschließen, dass sich dieser fundamentale Unterschied in der medialen Präsentation der Experimente auf die Schüler auswirkte. Was die Wirkung auf das Fachinteresse angeht, scheinen die Schüler der Kontroll- und Treatmentgruppe die Experimente in gleicher Weise rezipiert zu haben.

Der Rückgang des Fachinteresses Physik erfolgte in beiden Schuljahren synchron zum Rückgang des Interesses in anderen Fächern. Wie auch im Fach Physik sank das Interesse im Fach Mathematik und Chemie, in den Fächern Deutsch und Biologie blieb es nahezu unverändert. Deshalb ist der Rückgang im Fachinteresse Physik möglicherweise nicht allein der Gestaltung des Physikunterrichts anzulasten, sondern steht im größeren Kontext. Die Erhebungszeiträume März bis Juni 2016 und Februar bis Mai 2017 liegen jeweils im zweiten Halbjahr. Denkbar ist, dass es eine Art Zweites-Halbjahr-Effekt gibt, der für rückgängige Interessenswerte innerhalb eines laufenden Schuljahres mitverantwortlich ist, oder einen Oberstufeneffekt, der die Interessenswerte beim Eintritt in die Oberstufe möglicherweise zunächst erhöht, im Laufe des Schuljahres aber wieder kontinuierlich abfallen lässt. Solche Effekte wurden jedoch in der didaktischen Forschung noch nicht untersucht und sind auch an dieser Stelle Mutmaßungen, die im Rahmen dieser Arbeit nicht im Blick stehen.

Die Gruppe der affektiven Lernmerkmale Motivation, Fachinteresse und Selbstkonzept bildet eine Einheit, die sich als Ganzes in Kontroll- und Treatmentgruppe signifikant anders entwickelt hat, wie die MANOVA gezeigt hat. Der Effekt lag mit $\eta_{p}{ }^{2}=.058 \mathrm{im}$ kleinen bis mittleren Bereich. In der Summe betrachtet wurde die Entwicklung der affektiven Lernmerkmale durch den Flipped Classroom positiv beeinflusst. Werden sie als Gruppe betrachtet, kann Hypothese $\mathrm{H}_{1} \mathrm{~b}$ angenommen werden, werden sie differenziert betrachtet, gilt $\mathrm{H}_{1} \mathrm{~b}$ nur für die Motivation und das Selbstkonzept, nicht aber für das Fachinteresse. H1a konnte für die Lernleistung uneingeschränkt bestätigt werden.

\subsection{Forschungsfrage 2: Einfluss von Leistungsniveau und Geschlecht}

Nachdem die erste Forschungsfrage die Haupteffekte des Flipped Classroom auf die abhängigen Variablen untersucht hat, will die zweite Forschungsfrage mögliche Interaktionseffekte mit anderen unabhängigen Variablen untersuchen. Die beiden Hypothesen $\mathrm{H} 2 \mathrm{a}$ und $\mathrm{H} 2 \mathrm{~b}$ postulieren, dass weder das physikbezogene Leistungsniveau noch das Geschlecht eine Rolle in der Wirkung der Flipped Classroom spielen. Des Weiteren wird innerhalb dieser Forschungsfrage auch der Blick auf den Einfluss der Lehrkräfte zur richten sein. 


\subsubsection{Hypothese 2a: Leistungsniveau}

Hza. Das Leistungsniveau im Fach Physik beeinflusst nicht die Wirkung des Flipped Classroom auf die Lernleistung, die Motivation, das Fachinteresse und das Selbstkonzept.

Die kognitive Lernausgangslage (vgl. Kap. 6.1.1) zeigte, dass die Schüler im Flipped Classroom aus nichtsystematischen Gründen im Halbjahreszeugnis bessere Physiknoten 11/1 hatten als die Schüler der Kontrollgruppe, sie also ein höheres mittleres Leistungsniveau besaßen. Es ist deshalb denkbar, dass der Flipped Classroom in der Treatmentgruppe zu einer höheren Lernleistung führte, weil diese Unterrichtsmethode in besonderer Weise leistungsstarke Schüler in ihrem Lernverhalten begünstigt und es mithin eine Interaktion von Leistungsniveau und Methode gibt. Dennoch wird, wie in Kap. 3 dargelegt, zunächst von der Nullhypothese ausgegangen, weil sich aus der aktuellen Forschungslage keine eindeutigen Argumente für eine Interaktion ableiten lassen.

Um das Leistungsniveau im Fach Physik als mehrstufigen Faktor in eine ANOVA aufzunehmen, werden die Schüler in drei Leistungsgruppen eingeteilt. Auf unterem Leistungsniveau (Notenpunkte o-6) befinden sich 40 Schüler (26.5\%), auf mittlerem Niveau (Notenpunkte 7-10) befinden sich 66 Schüler (43.5\%) und auf dem oberen Niveau (11-15 Punkte) befinden sich 44 Schüler (29.1\%). Erfahrungsgemäß bildet eine solche gaußnahe Verteilung das typische Leistungsspektrum in Schulklassen und -kursen ab (Ziegenspeck 1999, S. 120).

Die Lernzuwächse $g$ für die drei Untergruppen in der Kontroll- und Treatmentgruppe werden in Tab. 14 und Abb. 22 dargestellt. Eine zweifaktorielle ANOVA von Hakes $g$ als abhängige Variable für den Lernzuwachs mit zwei Faktoren als unabhängigen Variablen (Methode und Leistungsniveau) zeigt, dass das Leistungsniveau unabhängig von der Methode den entscheidenden Effekt für den Lernzuwachs ausmacht, was auf der Hand liegt (vgl. Tab. 15). Das Leistungsniveau ist der stärkste Prädiktor für den Lernzuwachs. Im Vergleich dazu besitzt die Methode in dieser ANOVA einen weitaus geringeren, aber immer noch signifikanten Effekt auf den Lernzuwachs. Es gibt also zwei Haupteffekte.

Tab. 14. Lernzuwachs (Hakes g) in Kontroll- und Treatmentgruppe differenziert nach kategorialem Leistungsniveau

\begin{tabular}{|c|c|c|c|c|c|c|}
\hline & \multicolumn{3}{|c|}{ traditionell } & \multicolumn{3}{|c|}{ flipped } \\
\hline & $N$ & $M$ & $S D$ & $N$ & $M$ & $S D$ \\
\hline unteres Leistungsniveau & 26 & .24 & .13 & 12 & .30 & .15 \\
\hline mittleres Leistungsniveau & 32 & .36 & .16 & 34 & .41 & .12 \\
\hline oberes Leistungsniveau & 20 & .53 & .14 & 24 & .61 & .16 \\
\hline
\end{tabular}




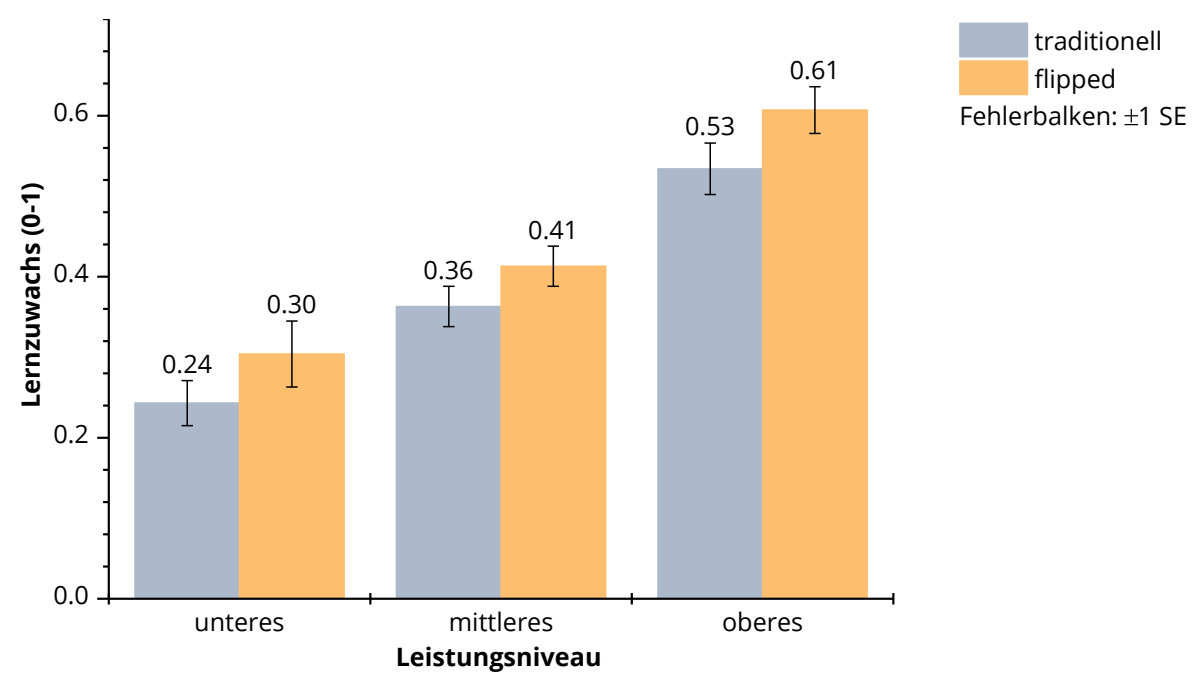

Abb. 22. Lernzuwachs für Schüler im unteren, mittleren und oberen Leistungsniveau

Des Weiteren zeigt sich, dass es keine signifikante Interaktion von Methode und Leistungsniveau gibt, $F_{\text {Meth }}{ }^{*}$ Leist $(2,142)=.084, p=.920$. Das bedeutet, dass der Effekt der Methode nicht durch das Leistungsniveau moderiert wird. Es macht für die Wirkung des Flipped Classroom auf die Lernleistung der Schüler keinen Unterschied, ob ein Schüler eher leistungsschwach, leistungsstark oder im mittleren Leistungsbereich ist. Flipped Classroom wirkt für Schüler relativ zu ihren Leistungsniveaus gleich gut.

Für die Variablengruppe Motivation, Interesse und Selbstkonzept zeigt eine MANOVA unter Verwendung der Pillai-Spur ebenfalls keinen Interaktionseffekt von Methode und Leistungsniveau, $V=.026, F(6,284)=.630, p=.706$. Für keinen F-Wert der drei Einzel-ANOVAs liegt die Signifikanz unter der Grenze von $\alpha=.05$ (vgl. Tab. 16). Auch für die affektiven Lernmerkmale gibt es keine Wechselwirkung zwischen Methode und Leistungsniveau.

Tab. 15. ANOVAs für Interaktionen von Methode und Leistungsniveau hinsichtlich des Lernzuwachses

\begin{tabular}{|lccccc|} 
& $d f$ & $d f$ Residuen & $F$ & $p$ & $\eta_{p}^{2}$ \\
\hline Methode & 1 & 142 & $\mathbf{5 . 9 6 8}$ & $\mathbf{. 0 1 6}$ & $\mathbf{. 0 4 0}$ \\
\hline Leistungsniveau & 2 & 142 & $\mathbf{4 2 . 6 6 5}$ & $<.001$ & .375 \\
\hline Methode x Leistungsniveau & 2 & 142 & .084 & .920 & .001
\end{tabular}

Tab. 16. Einfluss der Unterrichtsmethode und des Leistungsniveaus auf den Lernzuwachs (Hakes g)

\begin{tabular}{|lcccc|} 
& $d f$ & $d f$ Residuen & FMeth*Leist & $p$ \\
\hline$\Delta$ Motivation & 2 & 143 & .305 & .738 \\
\hline$\Delta$ Fachinteresse & 2 & 143 & .304 & .738 \\
\hline$\Delta$ Selbstkonzept & 2 & 143 & .734 & .482 \\
\hline
\end{tabular}


Da weder für die Lernleistung noch für die Motivation, das Fachinteresse oder das Selbstkonzept irgendeine Interaktion von Methode und Leistungsniveau signifikant ist, kann die Hypothese Hza uneingeschränkt angenommen werden. Für keine der abhängigen Variablen ist es im Flipped Classroom relevant, wie leistungsstark oder -schwach die Schüler sind. Flipped Classroom ist keine Methode, in der Schüler auf einem bestimmten Leistungsniveau einen besonderen Vor- oder Nachteil haben.

\subsubsection{Hypothese 2b: Geschlechtereffekt}

H2b. Das Geschlecht beeinflusst nicht die Wirkung des Flipped Classroom auf die Lernleistung, die Motivation, das Fachinteresse und das Selbstkonzept.

Im nächsten Schritt wird untersucht, ob es hinsichtlich der Wirkung des Flipped Classroom einen Geschlechtereffekt gibt, das heißt, ob diese Methode auf Mädchen anders wirkt als auf Jungen. Dazu gibt Tab. 17 nachfolgend - parallel zu Kap. 6.1 - einen Überblick, wie die Lernausgangslage für Jungen und Mädchen in den relevanten abhängigen Variablen ist und wo es gravierende Unterschiede zwischen den Geschlechtern gibt.

Der Levene-Test fällt für alle Variablen mit Ausnahme von Physiknote 1o. Klasse und Lehrerunterstützung pre nicht signifikant aus. Die Ergebnisse des Gruppenvergleichs sind für diese beiden Variablen kritisch zu sehen. In der Zusammenschau der signifikanten Ergebnisse lässt sich sagen: Hinsichtlich ihrer Leistungen im Fach Physik, ihres Fachinteresses und ihres Selbstkonzepts gibt es zwischen Jungen und Mädchen keine Unterschiede. Was das Selbstkonzept angeht, stehen die Ergebnisse nicht im Einklang mit durchgängigen Befunden, wonach - trotz gleicher Leistung - das physikbezogene Selbstkonzept von Mädchen niedriger als das von Jungen ist (Rost et al. 2006).

Signifikante Unterschiede gibt es zunächst in der physikbezogenen Motivation, welche bei den Mädchen niedriger ist als bei den Jungen (gemäß Krapp \& Prenzel 2011), und in der Mathematiknote 11/1, die bei den Mädchen höher ist. Auffällig ist aber besonders, dass die Mädchen signifikant höhere Werte in den fachunabhängigen Merkmalen besitzen, die unter dem Begriff Arbeitshaltung zusammengefasst werden (vgl. Kap. 5.4.2). Mädchen besitzen eine höhere Leistungsmotivation, neigen weniger zu Prokrastination, organisieren ihre Arbeit deutlich besser und arbeiten disziplinierter und länger an ihren Hausaufgaben. Diese Befunde stehen im Einklang mit anderen Studien zum schulischen Arbeitsverhalten (Wagner \& Spiel 2002). Die Arbeitshaltung der Mädchen, die Physik in der Oberstufe gewählt haben, ist deutlich besser als die ihrer männlichen Mitschüler. Für die Konstrukte Begeisterung für digitale Geräte und Selbstkonzept digitale Kompetenz besitzen die Jungen der gesamten Stichprobe wie erwartet höhere Werte (Christoph et al. 2015).

Lernleistung: Zunächst wird der Lernzuwachs betrachtet. In Tab. 18 finden sich die deskriptiven Daten sowie die Ergebnisse eines Teilgruppenvergleichs: Die Mädchen der Treatmentgruppe werden mit den Mädchen der Kontrollgruppe verglichen und die Jungen der Treatmentgruppe mit den Jungen der Kontrollgruppe. 
Tab. 17. Lernausgangslage differenziert nach fungen und Mädchen (aufgeführt sind die Fälle, von denen ein vollständiger Datensatz vorlag)

\begin{tabular}{|c|c|c|c|c|c|c|c|}
\hline & & \multicolumn{2}{|c|}{ Mädchen $(N=37)$} & \multicolumn{2}{|c|}{ Jungen ( $N=99)$} & \multirow[b]{2}{*}{$F$} & \multirow[b]{2}{*}{$p$} \\
\hline & & M & $S D$ & $M$ & $S D$ & & \\
\hline & Physiknote 11/1 (0-15) & 9.19 & 2.83 & 8.36 & 3.21 & 1.894 & .171 \\
\hline & Physiknote 10. Jgst. (1-6) & 2.16 & .76 & 2.40 & .93 & 2.014 & .158 \\
\hline & Mathematiknote 11/1 (0-15) & 10.68 & 2.40 & 9.48 & 3.06 & 4.558 & .035 \\
\hline & Motivation pre (0-3) & 1.37 & .68 & 1.67 & .71 & 5.036 & .026 \\
\hline & Fachinteresse pre (0-3) & 1.81 & .78 & 2.04 & .76 & 2.454 & .120 \\
\hline & Selbstkonzept pre (0-3) & 1.55 & .67 & 1.77 & .70 & 2.890 & .091 \\
\hline & Lehrerunterstützung pre (0-3) & 2.02 & .66 & 1.96 & .45 & .390 & .533 \\
\hline & Leistungsmotivation (0-3) & 2.15 & .54 & 1.72 & .65 & 14.114 & $<.001$ \\
\hline 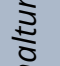 & Prokrastination, invers $(0-3)^{13}$ & 1.30 & .61 & 1.02 & .62 & 5.730 & .018 \\
\hline$\frac{2}{2}$ & Lernorganisation & 2.00 & .49 & 1.40 & .53 & 39.527 & $<.001$ \\
\hline & HA-Disziplin pre (0-3) & 1.86 & 1.03 & 1.16 & .89 & 15.422 & $<.001$ \\
\hline & HA-Dauer pre (0-3) & 1.68 & .88 & 1.15 & .94 & 8.635 & .004 \\
\hline & Begeisterung für digitale Geräte (0-3) & 1.84 & .35 & 2.00 & .46 & 4.092 & .045 \\
\hline & Selbstkonzept digitale Kompetenz (0-3) & 1.62 & .46 & 2.20 & .52 & 35.125 & $<.001$ \\
\hline
\end{tabular}

Schaut man sich nur die Mädchen an, so ist der Unterschied im Lernzuwachs zwischen Kontroll- und Treatmentgruppe signifikant. Doch auch für die Jungen ist der Unterschied zwischen Kontroll- und Treatmentgruppe signifikant. Sowohl Mädchen als auch Jungen haben also im Flipped Classroom signifikant bessere Lernleistungen im Vergleich zum traditionellen Unterricht, wie Abb. 23 illustriert.

Eine zweifaktorielle ANOVA mit den beiden unabhängigen Faktoren Methode und $\mathrm{Ge}$ schlecht zeigt zunächst, dass das Geschlecht selbst keinen signifikanten Einfluss auf den Lernzuwachs hat (vgl. Tab. 19). Im Gegensatz dazu ist der Einfluss der Methode - wie bisher schon deutlich wurde - signifikant. Es gibt unabhängig von der gewählten Methode also keinen leistungsmäßigen Unterschied zwischen Mädchen und Jungen im Physikunterricht. Mädchen schneiden im Leistungstest nicht besser oder schlechter ab als Jungen. Dieser Befund passt

13 Diese Skala ist invers, das bedeutet: o: starke Prokrastination, 3: schwache Prokrastination. 
Tab. 18. Lernzuwachs in Kontroll- und Treatmentgruppe differenziert nach Mädchen und fungen

\begin{tabular}{|c|c|c|c|c|c|c|c|c|c|}
\hline & \multicolumn{3}{|c|}{ traditionell } & \multicolumn{3}{|c|}{ flipped } & \multirow[b]{2}{*}{$F$} & \multirow[b]{2}{*}{$p$} & \multirow[b]{2}{*}{$\eta_{p^{2}}$} \\
\hline & $N$ & $M$ & $S D$ & $N$ & $M$ & $S D$ & & & \\
\hline Mädchen (Hakes g) & 17 & .36 & .19 & 23 & .50 & .17 & 5.860 & .020 & .134 \\
\hline Jungen (Hakes g) & 62 & .37 & .18 & 47 & .44 & .18 & 4.267 & .041 & .038 \\
\hline
\end{tabular}

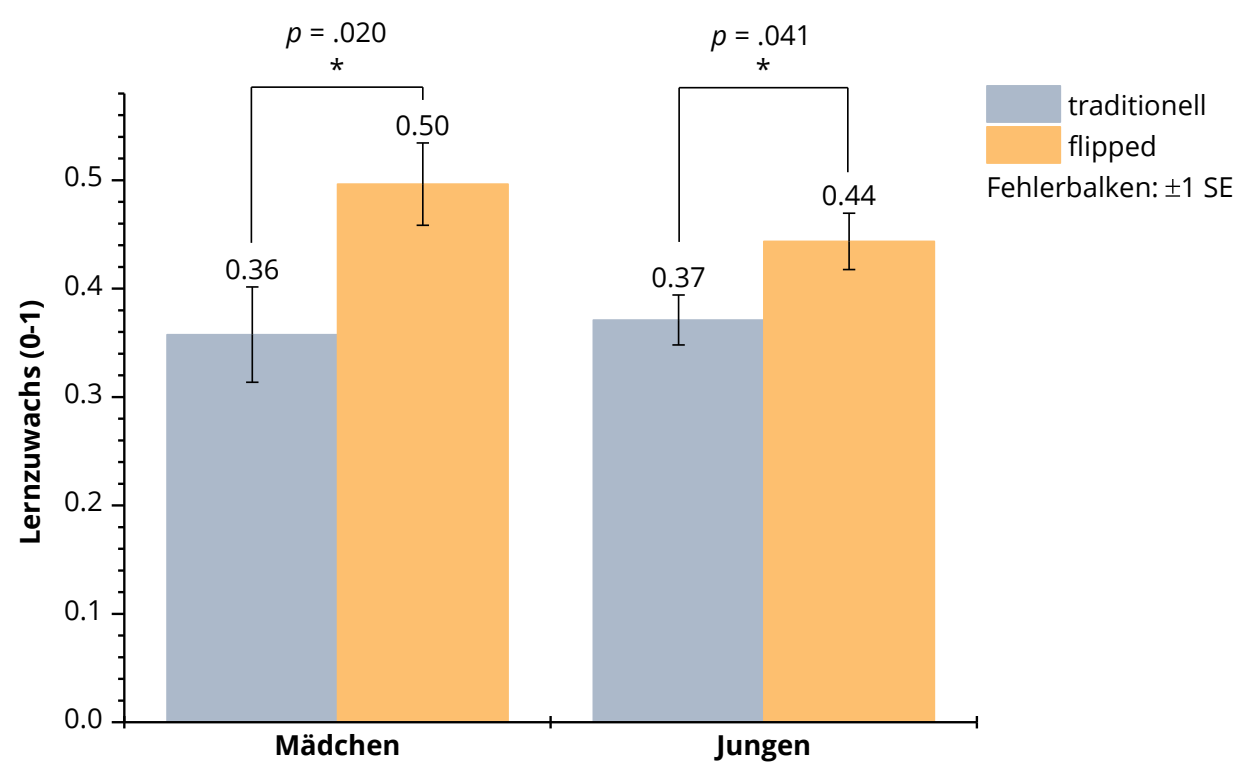

Abb. 23. Lernzuwachs (Hakes g) in Kontroll- und Treatmentgruppe differenziert nach Geschlecht

grundsätzlich in die traditionelle Forschungslage (Häussler \& Hoffmann 1998; Prenzel et al. 2007), auch wenn die Erhebungen im Rahmen von PISA 2015 eine größere Leistungskluft zwischen Jungen und Mädchen feststellen, als allgemein angenommen wird (Schiepe-Tiska et al. 2016).

Vor allem aber zeigt sich, dass es keine Interaktion von Methode und Geschlecht gibt, $F_{M e t h}{ }^{*} \operatorname{Geschl}(1,145)=.964, p=.328$. Flipped Classroom wirkt leistungsmäßig auf Mädchen nicht anders als auf Jungen. Kein Geschlecht besitzt in dieser Methode gegenüber dem anderen einen Vorteil. Bezüglich des Lernzuwachses gibt es im Flipped Classroom keinen Geschlechtereffekt. In dieser Hinsicht wird $\mathrm{H}_{2} \mathrm{~b}$ angenommen.

Tab. 19. Einfluss der Unterrichtsmethode und des Geschlechts auf den Lernzuwachs (Hakes g)

\begin{tabular}{|c|c|c|c|c|c|}
\hline & $d f$ & df Residuen & $F$ & $p$ & $\eta p^{2}$ \\
\hline Methode & 1 & 145 & 9.771 & .002 & .063 \\
\hline Geschlecht & 1 & 145 & .340 & .561 & .002 \\
\hline Methode x Geschlecht & 1 & 145 & .964 & .328 & .007 \\
\hline
\end{tabular}


Motivation, Fachinteresse, Selbstkonzept: Eine MANOVA mit den beiden unabhängigen Faktoren Methode und Geschlecht zeigt unter Verwendung der Pillai-Spur für die Variablengruppe Motivation, Fachinteresse und Selbstkonzept einen Haupteffekt, der - wie bereits in Kap. 6.2.2 gesehen - bei der Methode liegt, $V=.117, F(3,144)=6.366, p<.001, \eta_{p}^{2}=.117$. Das Geschlecht besitzt allein, wie schon beim Lernzuwachs, keinen Einfluss auf die Variablengruppe, $V=.008, F(3,144)=.377, p=.770$. Jedoch ist für die Gruppe der affektiven Lernmerkmale eine Interaktion von Methode und Geschlecht auszumachen, $V=.096, F(3,144)=5.104$, $p=.002, \eta_{p}{ }^{2}=.096$. Der Effekt liegt im mittleren Bereich. Der Flipped Classroom wirkt bei den Mädchen auf die affektiven Lernmerkmale anders als bei den Jungen. Die nachfolgenden Einzel-ANOVAs in Tab. 20 zeigen, dass vor allem in der Änderung der Motivation das Geschlecht und die Methode zusammenwirken.

Für sich genommen sind die Unterschiede in den Gain Scores des Fachinteresses und des Selbstkonzepts nicht signifikant. Erst im Verbund miteinander und dem Gain Score der Motivation gewinnt die Interaktion von Methode und Geschlecht an Bedeutung. Bei einem Interaktionseffekt mittlerer Stärke $\left(\eta_{p}{ }^{2}=.070\right)$ lohnt es sich, die Motivationsentwicklung etwas genauer anzuschauen. Offenbar gibt es hier deutliche Unterschiede für Mädchen im traditionellen Unterricht und im Flipped Classroom (vgl. Tab. 21).

Im Gegensatz zu jeder anderen Untergruppe verbesserte sich bei den Mädchen im Flipped Classroom die physikbezogene Motivation. Betrachtet man die Teilmenge der Mädchen in der Gesamtstichprobe, ist der Zuwachs an Motivation im Flipped Classroom gegenüber dem traditionellen Unterricht signifikant, $F(1,38)=17.843, p<.001, \eta_{p}{ }^{2}=.320$. Für die fungen hingegen macht die Wahl der Unterrichtsmethode motivational keinen signifikanten Unterschied. Betrachtet man alle Schüler im Flipped Classroom, gibt es keinen Geschlechtereffekt.

Tab. 20. Interaktionen von Methode und Geschlecht hinsichtlich der affektiven Lernmerkmale

\begin{tabular}{|lccccc} 
& $d f$ & $d f$ Residuen & $F_{\text {Meth }{ }^{*} \text { Geschl }}$ & $p$ & $\eta_{p}{ }^{2}$ \\
\hline$\Delta$ Motivation & 1 & 146 & $\mathbf{1 0 . 9 2 8}$ & $\mathbf{. 0 0 1}$ & $\mathbf{. 0 7 0}$ \\
\hline$\Delta$ Fachinteresse & 1 & 146 & 1.043 & .309 & .007 \\
\hline$\Delta$ Selbstkonzept & 1 & 146 & 2.393 & .124 & .016
\end{tabular}

Tab. 21. Motivationsentwicklung (post - pre) in Kontroll- und Treatmentgruppe differenziert nach Mädchen und Jungen

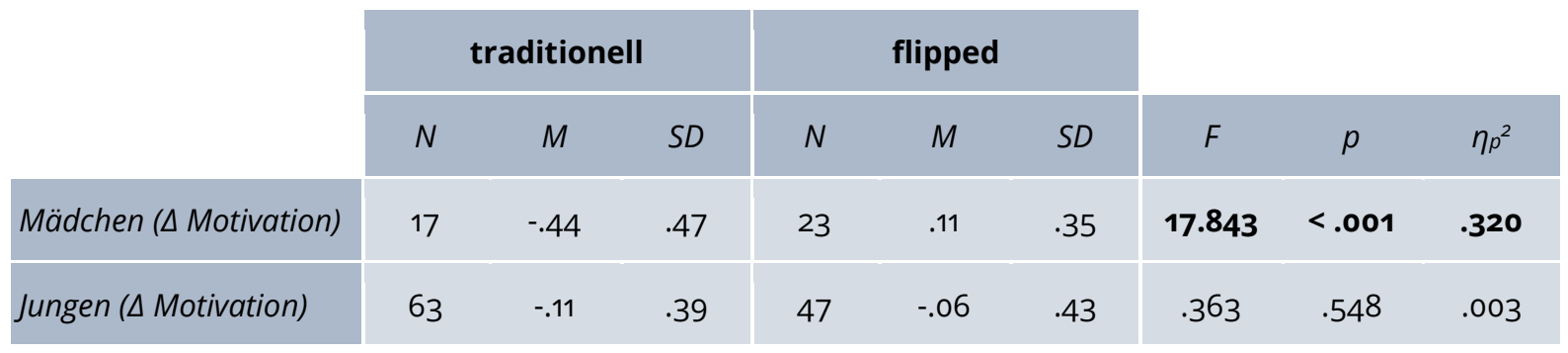




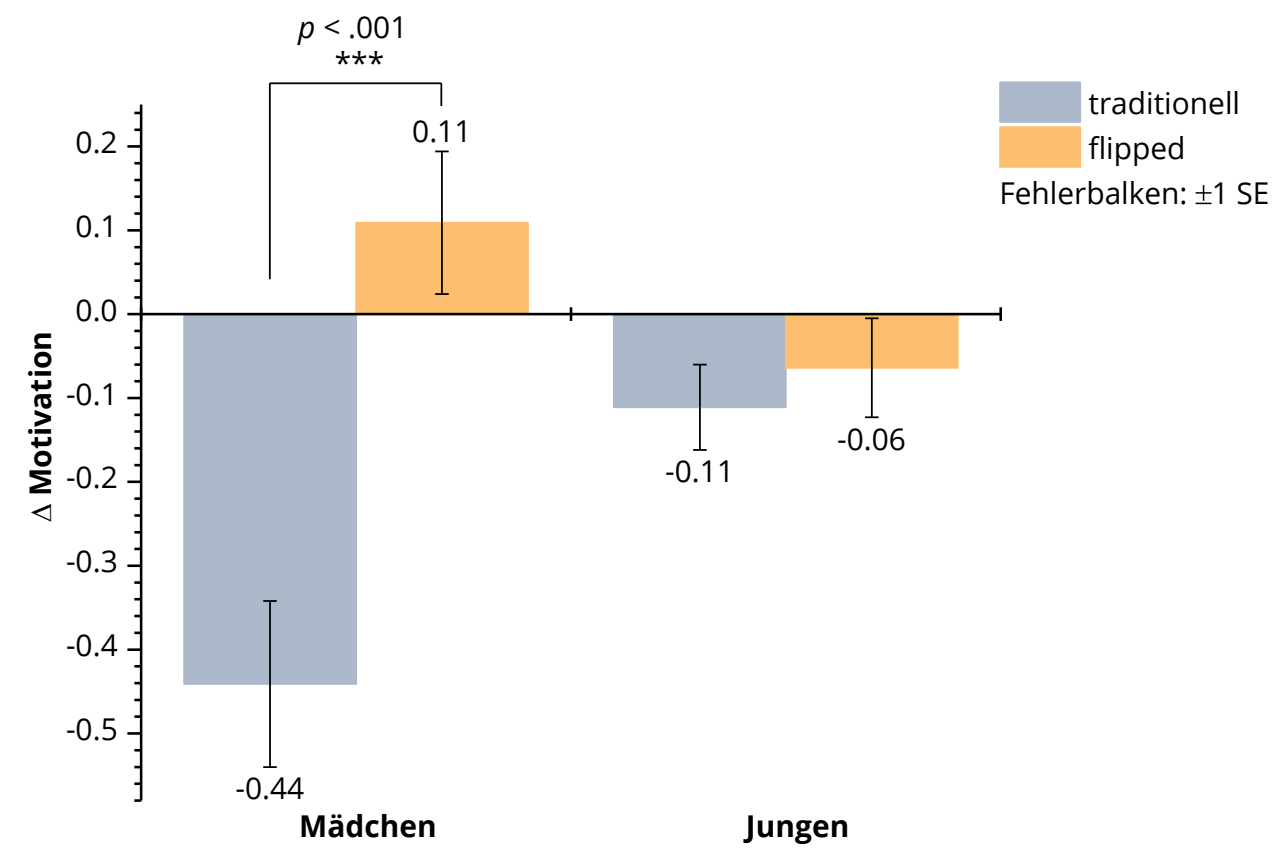

Abb. 24. Motivationsentwicklung in Kontroll- und Treatmentgruppe differenziert nach Mädchen und Jungen

Das zugehörige Diagramm Abb. 24 illustriert den deutlichen Unterschied in der Motivationsentwicklung der Mädchen im Flipped Classroom. der Unterschied in der Motivationsentwicklung bei Mädchen und Jungen, die alle im Flipped Classroom unterrichtet wurden, liegt oberhalb der Signifikanzgrenze von $\alpha=.05, F_{f l i p}(1,68)=2.739, p=.103$.

Die deskriptiven Daten im Eingang dieses Unterkapitels (vgl. Tab. 17) zeigen, dass die Mädchen vor Beginn der Unterrichtsreihe eine deutlich niedrigere Motivation als die Jungen hatten $\left(M_{\text {Mädchen }}=1.37, S D=.68\right.$ vs. Mfungen $\left.=1.67, S D=.71\right)$. Im traditionellen Unterricht hat sich diese noch einmal deutlich verschlechtert (auf $M_{\text {Mädchen }}=1.06, S D=.87$ ). Im Flipped Classroom hingegen kam es für die Mädchen zu einer signifikanten Steigerung der Motivation. In Kap. 6.2.2 wurde deutlich, dass im Flipped Classroom die Motivation die größte Steigerung von den affektiven Lernmerkmalen erfuhr. Unter Einbezug des Geschlechts zeigt sich, dass es insbesondere die Mädchen sind, die für diese Steigerung verantwortlich sind. Bei ihnen ändert sich die Motivation am deutlichsten zu positiven Werten.

Arbeitshaltung: In der Lernausgangslage (vgl. Tab. 17) wird deutlich, dass sich die Mädchen gegenüber den Jungen durch eine signifikant bessere Arbeitshaltung (Leistungsmotivation, Lernorganisation, inverse Prokrastination, Hausaufgabendisziplin) auszeichnen. Eine multiple Regression soll untersuchen, ob die Motivationssteigerung im Flipped Classroom möglicherweise eher von der Arbeitshaltung moderiert wird, indem die stärksten Prädiktoren für die Motivationssteigerung identifiziert werden (Tab. 22). In das Modell gehen alle oben genannten Faktoren der Arbeitshaltung (Leistungsmotivation, Lernorganisation, inverse Prokrastination, Hausaufgabendisziplin) und ihre Interaktionen mit der Methode in eine Regression ein. Der Gain Score $\Delta$ Motivation ist die abhängige Variable. 
Tab. 22. Regressionsanalyse der Motivationsentwicklung

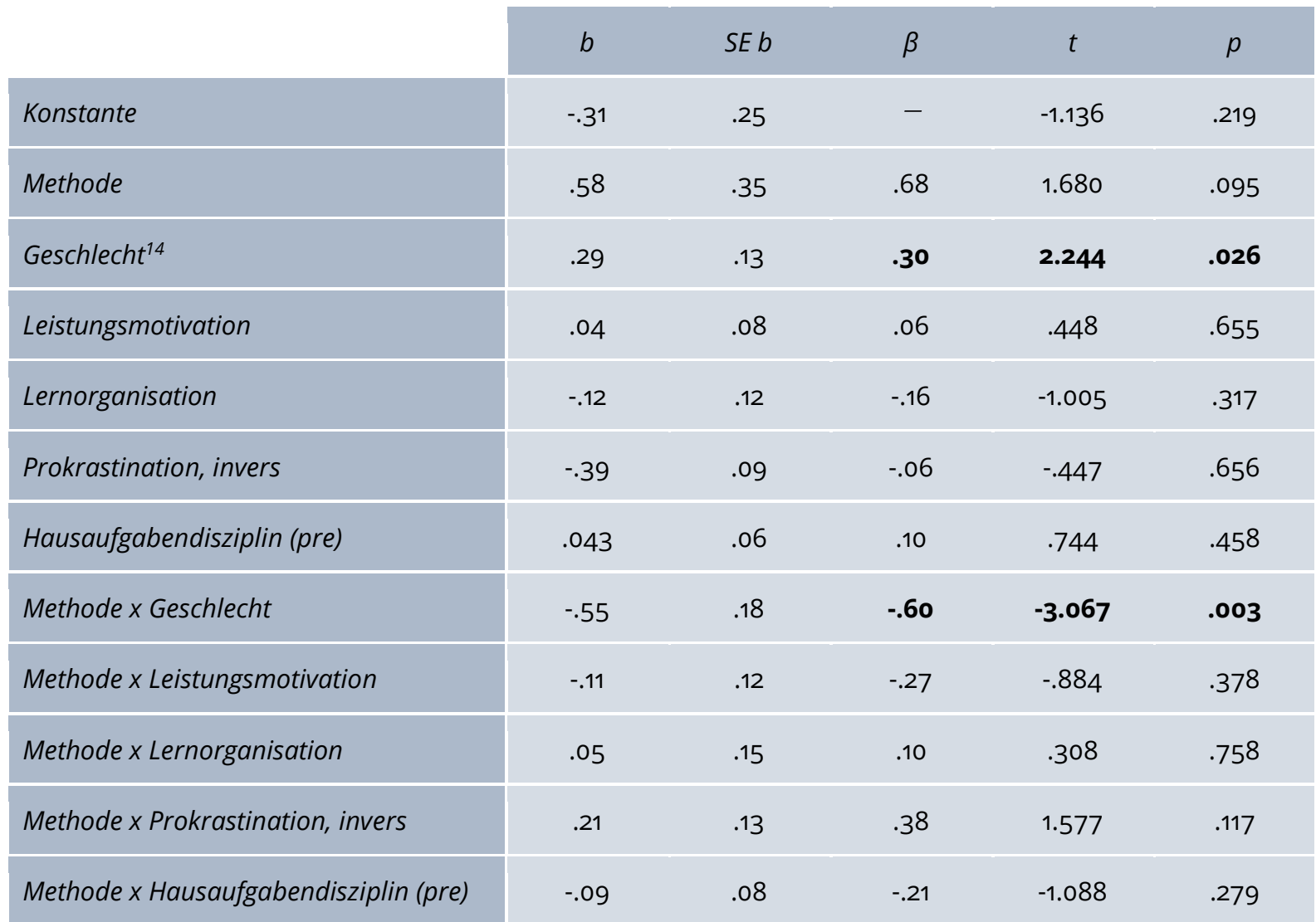

Anmerkung: $R^{2}=.074$

Das Modell klärt nicht viel Varianz auf $\left(R^{2}=.074\right)$, aber der stärkste Prädiktor für die Änderung der Motivation ist die Interaktion von Methode und Geschlecht, $\beta=-.60, p=.003$. Ansonsten ist in dem Modell nur noch das Geschlecht als Haupteffekt signifikant, $\beta=.30$, $p=.026$. Der $\beta$-Koeffizient ist positiv, was bedeutet, dass es sich hier um einen Motivationsverlust handelt, das Geschlecht also als einzelner Faktor ein Prädiktor ist. In der Interaktion mit der Methode ist der $\beta$-Koeffizient negativ, was hier für einen Motivationszuwachs bei Mädchen steht. ${ }^{14}$ Keine weiteren Faktoren oder Interaktionen tragen signifikant zur Motivationsentwicklung bei, so dass eine Moderation durch einen anderen Faktor als das Geschlecht tatsächlich vernachlässigt werden kann. Es lag nicht an der besseren Arbeitshaltung der Mädchen, dass es im Flipped Classroom zu einer so positiven Entwicklung ihrer Motivation kam, sondern - gemäß der Regressionsanalyse - an der Tatsache, dass sie Mädchen sind.

Beim Blick auf geschlechterspezifische Differenzen in der Lernausgangslage fiel auf, dass die Jungen eine stärker ausgeprägte Begeisterung für digitale Geräte und ein besseres Selbstkonzept digitale Kompetenz besaßen, auch wenn dies nach Forschungslage nicht mit höheren Fähigkeiten im Bereich der Internet- und Kommunikationstechnologie korreliert (Christoph

${ }^{14}$ Die Kodierung ist: weiblich=0, männlich=1. 
et al. 2015). Eine Erklärung für den motivationalen Gewinn der Mädchen im Flipped Classroom lässt sich aus ihren niedrigeren affektiven Werten zur Internet- und Kommunikationstechnologie jedoch nicht generieren.

\subsubsection{Einfluss der Lehrkraft}

Da die Forschungsfrage 2 den Einflüssen weiterer Faktoren auf die Wirksamkeit des Flipped Classroom nachgeht, soll in diesem Kontext noch ansatzweise der Einfluss der unterrichtenden Lehrer untersucht werden. Für eine explizite Untersuchung der Rolle der Lehrkraft bedarf es einer bedeutend umfangreicheren Stichprobe und einer größeren Anzahl von Lehrern. Deshalb liegt auf der Frage nach dem Einfluss des Lehrers in dieser Studie kein Hauptaugenmerk, sondern sie soll die bisherigen Befunde nur flankieren.

An der Studie nahmen nur fünf Lehrkräfte teil, lediglich drei von ihnen konnten aus schulorganisatorischen Gründen beide Methoden unterrichten (vgl. Kap. 5.1.2). Um zu untersuchen, welchen Einfluss die Lehrkraft auf die kognitiven und affektiven Lernergebnisse der Schüler im Flipped Classroom hat, werden deshalb nur die drei Lehrer 1-3 von Schule A betrachtet, die sowohl einen Kontroll- als auch einen Treatmentkurs unterrichtet haben. Dadurch verringert sich der Stichprobenfang von $N_{\text {total }}=151$ auf $N_{\text {red }}=121$. Wie bereits erwähnt, waren alle an der Studie beteiligten Lehrkräfte männlich. Mögliche Effekte, die durch Geschlechterunterschiede bei den Lehrkräften hervorgerufen sein könnten, lassen sich damit ausschließen. An dieser Stelle soll in Erinnerung gerufen werden, dass der Autor selbst einer der drei Lehrkräfte der Schule A war (vgl. Kap. 5.1.2).

Lernleistung: Auch in der reduzierten Stichprobe ist der Levene-Test nicht signifikant ( $p=.846$ ). Aus Abb. 25 werden die Lernzuwächse aufgeteilt nach Lehrer 1-3 ersichtlich. Die Ergebnisse einer ANOVA mit dem Lernzuwachs als abhängiger Variable und der Methode und Lehrer als unabhängigen festen Faktoren zeigt Abb. 25.

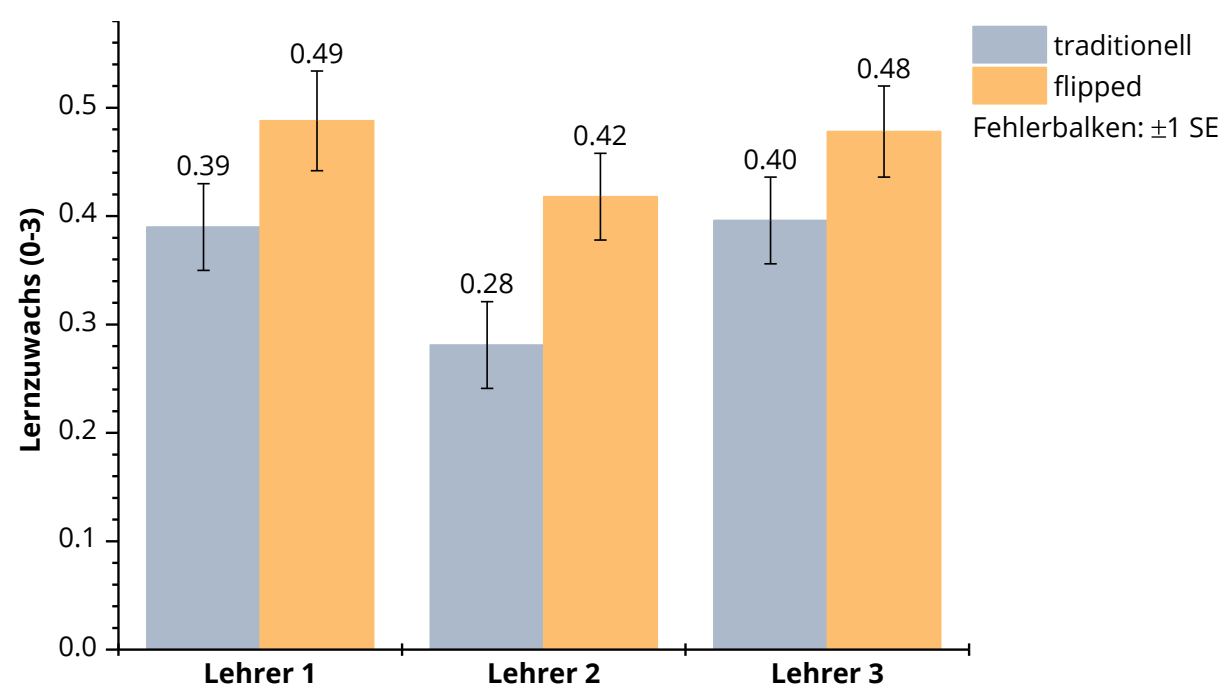

Abb. 25. Lernzuwachs (Hakes g) nach Lehrkräften 
Tab. 23. Einfluss der Unterrichtsmethode und der Lehrkraft auf den Lernzuwachs (Hakes g)

\begin{tabular}{|lccccc} 
& $d f$ & df Residuen & $F$ & $p$ & $\eta_{p}{ }^{2}$ \\
\hline Methode & 1 & 113 & $\mathbf{9 . 9 3 7}$ & .002 & .081 \\
\hline Lehrer & 1 & 113 & $\mathbf{3 . 1 8 6}$ & .045 & .053 \\
\hline Methode x Lehrer & 1 & 113 & .245 & .783 & .004
\end{tabular}

In der reduzierten Stichprobe mit diesen drei Lehrkräften wird deutlich, dass die Methode unabhängig vom unterrichtenden Lehrer - zu einem höheren Lernzuwachs führt als der traditionelle Unterricht. Es zeigt sich auch, dass der einzelne Lehrer, unabhängig von der Methode, einen signifikanten Einfluss auf den Lernzuwachs der Schüler besitzt, das bedeutet, dass einzelne Lehrer allein durch ihr unterrichtliches Handeln ihre Schüler zu anderen Leistungen führen können als andere Lehrer - unabhängig von der Unterrichtsmethode. Vor allem aber gibt es keine signifikante Interaktion von Methode und Lehrer, das heißt, dass es hinsichtlich ihres Lernzuwachses für die Schüler keine Rolle gespielt hat, welcher Lehrer im Flipped Classroom unterrichtet hat - oder welcher Lehrer traditionell. Die lernförderlichere Wirkung des Flipped Classroom wurde nicht zusätzlich durch den Lehrer beeinflusst.

Diese Befunde dürfen angesichts der kleinen Gruppe von nur drei Lehrern jedoch nicht überbewertet werden. Offenbar haben alle drei Lehrer die Methode auf sehr vergleichbare Weise in ihrem Unterricht umgesetzt. Kein Lehrer hat den Flipped Classroom in einer Weise implementiert, dass die Schüler seines Kurses begünstigt oder benachteiligt gegenüber den Schülern der anderen Kurse wären. Die Unterschiede im Lernzuwachs zwischen den Treatmentkursen kommen ausschließlich durch die je verschiedene Lehrerpersönlichkeit zustande.

Motivation, Fachinteresse, Selbstkonzept: Betrachtet man die Änderungen der affektiven Lernmerkmale als Variablengruppe in einer MANOVA mit Methode und Lehrer als festen Faktoren unter Verwendung der Pillai-Spur, gibt es - wie schon in Kap. 6.2.2 gesehen - einen signifikanten Einfluss der Methode auf die Variablengruppe zugunsten des Flipped Classroom, $V=.083, \mathrm{~F}(3,112)=3.358, p=.021, \eta_{p}{ }^{2}=.083$. Der Lehrer selbst bewirkt keine Veränderung der affektiven Lernmerkmale, $V=.016, F(3,112)=.304, p=.934$, was nicht überrascht, da diese Variable für die Schüler ja konstant blieb. Interessant ist vielmehr, dass es keine Interaktion von Methode und Lehrer gibt, $V=.018, F(6,226)=1.583, p=.153$. Wie schon beim Lernzuwachs spielte es für die affektiven Lernmerkmale keine Rolle, welcher Lehrer das Treatment durchführte. Unabhängig von der unterrichtenden Lehrkraft hat sich die Gruppe der affektiven Lernmerkmale Motivation, Fachinteresse und Selbstkonzept in der Treatmentgruppe allein durch die Methode Flipped Classroom verbessert.

Die nachfolgenden Einzel-ANOVAs zeigen in Tab. 24, dass es bei keinem einzelnen affektiven Lernmerkmal eine signifikante Wechselwirkung von Methode und Lehrer gab - wenn, dann am ehesten bei der Motivation, bei der die Interaktion nur knapp nicht signifikant ist. 
Tab. 24. Interaktionen von Methode und Lehrer hinsichtlich der affektiven Lernmerkmale

\begin{tabular}{|lccccc} 
& $d f$ & $d f$ Residuen & $F_{\text {Meth*Lehrer }}$ & $p$ & $\eta_{p}{ }^{2}$ \\
\hline$\Delta$ Motivation & 2 & 114 & 2.768 & .067 & .046 \\
\hline$\Delta$ Fachinteresse & 2 & 114 & .995 & .373 & .017 \\
\hline$\Delta$ Selbstkonzept & 2 & 114 & 1.468 & .235 & .025 \\
\hline
\end{tabular}

Eine weiterführende Varianzanalyse, in der die Methode, das Geschlecht und die Lehrkraft als feste Faktoren eingehen und die eine mögliche Dreifachwechselwirkung aufdecken könnte, lässt sich mit der gegebenen Stichprobe nicht realisieren.

\subsubsection{Diskussion}

Leistungsniveau: Flipped Classroom wirkte sich in der Studie positiv auf die Lernleistung der Schüler aus, unabhängig von ihrem Leistungsniveau. Leistungsschwache Schüler profitierten ebenso von der Methode wie leistungsstarke. Bei den affektiven Lernmerkmalen Motivation, Fachinteresse und Selbstkonzept besaß das Leistungsniveau der Schüler ebenfalls keinen Einfluss auf die Wirkung des Flipped Classroom. Dieser Befund bestätigt, dass das Leistungsniveau der Schüler keine moderierende Funktion für den kognitiven und affektiven Lernerfolg hat. Flipped Classroom ist demnach eine breitbandig schüleraktivierende Methode. Die Möglichkeit zu innerer Differenzierung und Selbstbestimmung sowie die individuelle Lernbegleitung im Unterricht kommen den leistungsstarken Schülern ebenso zugute wie den leistungsschwachen. Der Befund der Studie widerspricht den Ergebnissen von Marlowe (2012), in dessen Studie besonders für leistungsschwache Schüler ein überdurchschnittlicher Lernzuwachs verzeichnet wurde, ${ }^{15}$ stützt aber die Ergebnisse der Hochschulstudie von Goerres et al. (2015), die ebenfalls keine Interaktion mit dem Leistungsvermögen feststellen konnten.

Geschlecht: Hypothesenkonform ließ sich feststellen, dass das Geschlecht im Flipped Classroom keinen moderierenden Einfluss auf die Lernleistung ausübte. Allerdings überraschte, dass es einen Geschlechtereffekt in den affektiven Lernmerkmalen gab. Mädchen im Flipped Classroom profitierten auf diesem Feld mehr als die Jungen. Den größten affektiven Lernerfolg hatten sie in der physikbezogenen Motivation. Die Motivation und Freude, sich auch außerhalb der Schule freiwillig mit physikalischen Themen zu beschäftigen, nahm bei den Mädchen im Flipped Classroom verglichen mit den Jungen und der Kontrollgruppe deutlich zu. Der Befund ist deshalb überraschend, weil der Flipped Classroom zunächst eine do-

15 Mit N = 19 Schülern war die Stichprobe in der Studie von Marlowe (2013) jedoch sehr klein. 
mänenfreie, generische Methode ist, die ihren Fokus nicht darauf richtet, Interesse oder Motivation von Mädchen hinsichtlich der Physik zu fördern. Wie ist dieses Ergebnis zu interpretieren?

Gegeben der Stichprobengröße - insgesamt nahmen 40 Mädchen an der Studie teil, davon waren 23 in der Treatmentgruppe - darf man diesen Befund zunächst nicht überbewerten, zumal das Fachinteresse Physik nicht in gleicher Weise zunahm wie die Motivation. Sucht man nach lernrelevanten Dispositionsunterschieden, zeigt sich, dass sich die Mädchen von den Jungen am deutlichsten durch ihre höhere Leistungsmotivation, ihr schwächer ausgeprägtes Prokrastinationsverhalten, ihre bessere Lernorganisation und ihre stärkere Hausaufgabendisziplin unterscheiden. Aber diese nachgewiesen bessere Arbeitshaltung der Mädchen kann nicht als Erklärung für den motivationalen Geschlechterunterschied herangezogen werden. Das zeigte die Regressionsanalyse. Das Sachinteresse und die Freude, sich mit Physik zu beschäftigen, wuchsen bei den Mädchen im Flipped Classroom nicht aufgrund ihrer deutlich stärker ausgeprägten Arbeitshaltung. Ein Ansatzpunkt für eine Erklärung findet sich möglicherweise im geschlechtstypischen Lernstil von Mädchen. Herzog (1996) führt sieben Kriterien für einen mädchengerechten Physikunterricht an, die Grundlage der Schweizer Koedukationsstudie waren. Eines der Kriterien orientiert sich an dem besonderen Lern- und Arbeitsstil von Mädchen. Demnach passen kooperative Lernformen mit ausreichender Zeit zum Lösen von Aufgaben - Hauptcharakteristika des Flipped Classroom - besonders gut zum aufgabenorientierten Lernstil von Mädchen. Die von Herzog (1996) ergänzte Forderung von geschlechtshomogenen Gruppenarbeiten fand zwar in der vorliegenden Studie keine Anwendung. Doch kann trotzdem in den ausgeprägten Phasen des kooperativen Lernens, das sonst im Physikunterricht der Oberstufe zu kurz kommt, ein möglicher Hinweis zur Aufklärung gefunden werden, warum Mädchen im Flipped Classroom einen höheren motivationalen Lerngewinn erzielen konnten. Für Folgestudien bieten sich hier Anknüpfungsmöglichkeiten. Die Forschungshypothese Hzb, nach der das Geschlecht die Wirkung des Flipped Classroom nicht beeinflusst, kann also nur für die kognitiven, nicht aber für die affektiven Lernergebnisse angenommen werden.

Lehrkraft: Das letzte Ergebnis, das im Rahmen der Forschungsfrage 2 gefunden wurde, soll ebenfalls diskutiert werden: der Einfluss der Lehrer. Für die Wirksamkeit des Flipped Classroom spielte es keine Rolle, welcher Lehrer in den Kursen unterrichtete. Zwar beeinflusste die Person des Lehrers direkt die Lernleistung, doch zeigte sich weder für die kognitiven noch für die affektiven Lernergebnisse eine Interaktion von Lehrer und Methode. Die Auswirkung des Flipped Classroom war damit unabhängig vom unterrichtenden Lehrer.

Aufgrund der geringen Zahl der Lehrer, die für die Frage nach dem Lehrereinfluss im Flipped Classroom zur Verfügung standen, darf dieser Befund nicht vorschnell generalisiert werden. Das Ergebnis, dass keine Interaktion festgestellt wurde, ist bei einer kleinen Stichprobe von nur drei Lehrern nicht sehr aussagekräftig. Dennoch zeigt die ausbleibende Interaktion von Lehrer und Methode, dass der Flipped Classroom von den beteiligten Lehrern der Schule A auf sehr vergleichbare Weise in ihren Kursen umgesetzt wurde. Voraussetzung dafür waren enge Absprachen und die konsequente Einhaltung des Methodenkonzepts, das in Kap. $5 \cdot 3$ 
dargestellt wurde. Sicher haben auch die Lehrerfortbildungen zum Flipped Classroom, durch deren Inhalte die beteiligten Lehrkräfte vorab geschult wurden, dazu beigetragen, ein einheitliches Verständnis davon zu haben, worauf zu achten ist und welche Veränderung im Lehrerverhalten der Flipped Classroom erfordert. So war ein für die Schüler aller Kurse einheitliches Treatment möglich, das die Basisform des Flipped Classroom, wie sie in dieser Studie umgesetzt wurde, vom Lehrer ausreichend unabhängig machte. Bei allen sonstigen Problemen interner Validität, die eine quasiexperimentelle Studie im Feld mit sich bringt, ist die ausbleibende Interaktion von Lehrer und Methode - selbst bei nur drei zu berücksichtigenden Lehrkräften - ein Argument für die Testgüte der Untersuchung.

\subsection{Forschungsfrage 3: Veränderung der Lehrerunterstützung und der Hausaufgabenbearbeitung}

Die dritte Forschungsfrage richtet sich auf Stützfaktoren des Unterrichts, also die Lehrerunterstützung und die Hausaufgabenbearbeitung. Auch hier wird für beide Forschungshypothesen zusätzlich geprüft, ob der Lehrer einen moderierenden Einfluss auf die Entwicklung der Stützfaktoren hatte. Diese Frage steht jedoch nicht im Vordergrund.

\subsubsection{Hypothese 3a: Lehrerunterstützung}

Hза. Der Flipped Classroom erhöht die von den Schülern wahrgenommene Lehrerunterstützung.

Die Lehrerunterstützung wurde mit einer Skala von 5 Items gemessen, die eine zufriedenstellende Reliabilität besitzt (vgl. Kap. 5.4.2). Beispielitems sind: »Unter Physiklehrer interessiert sich für den Lernfortschritt jedes einzelnen Schülers. « und »Unser Physiklehrer unterstützt uns zusätzlich, wenn wir Hilfe brauchen. «Der Shapiro-Wilk-Test des Gain Scores $\Delta$ der Lehrerunterstützung fällt für die Kontrollgruppe signifikant aus ( $p=.019)$, für die Treatmentgruppe hingegen nicht. Die Analysen beziehen sich hier zunächst auf die gesamte Stichprobe. Wie bereits in der Beschreibung der Lernausgangslage Tab. 17 gezeigt, ist die wahrgenommene Lehrerunterstützung in den Kursen der Treatmentgruppe vor Beginn der Studie höher als zum gleichen Zeitpunkt in der Kontrollgruppe.

Aus Tab. 25 ist die Änderung des Stützfaktors Lehrerunterstützung in beiden Gruppen zu ersehen, welche in Abb. 26 illustriert ist. Vergleicht man die Gain Scores miteinander, gibt es keinen signifikanten Unterschied in der Entwicklung der Lehrerunterstützung. In der Kontrollgruppe wird der Mittelwert leicht niedriger, in der Treatmentgruppe leicht höher, ohne dass innerhalb einer Gruppe oder zwischen den Gruppen ein signifikanter Unterschied sichtbar wird - im Prinzip bleiben die Werte für die Lehrerunterstützung in beiden Gruppen unverändert. Überraschend ist vor allem, dass die Schüler im Flipped Classroom keine stärkere Lehrerunterstützung wahrgenommen haben als zuvor. Sie fühlten sich im Flipped Classroom 
Tab. 25. Vergleich der Änderungen in der Lehrerunterstützung in Kontroll- und Treatmentgruppe

\begin{tabular}{|c|c|c|c|c|c|c|c|c|c|}
\hline & \multicolumn{3}{|c|}{ traditionell } & \multicolumn{3}{|c|}{ flipped } & \multirow[b]{2}{*}{$F$} & \multirow[b]{2}{*}{$p$} & \multirow[b]{2}{*}{$\eta p^{2}$} \\
\hline & $N$ & $M$ & $S D$ & $N$ & $M$ & $S D$ & & & \\
\hline$\Delta$ Lehrerunterstützung & 80 & -.018 & .401 & 70 & .024 & .445 & .353 & .553 & .002 \\
\hline
\end{tabular}
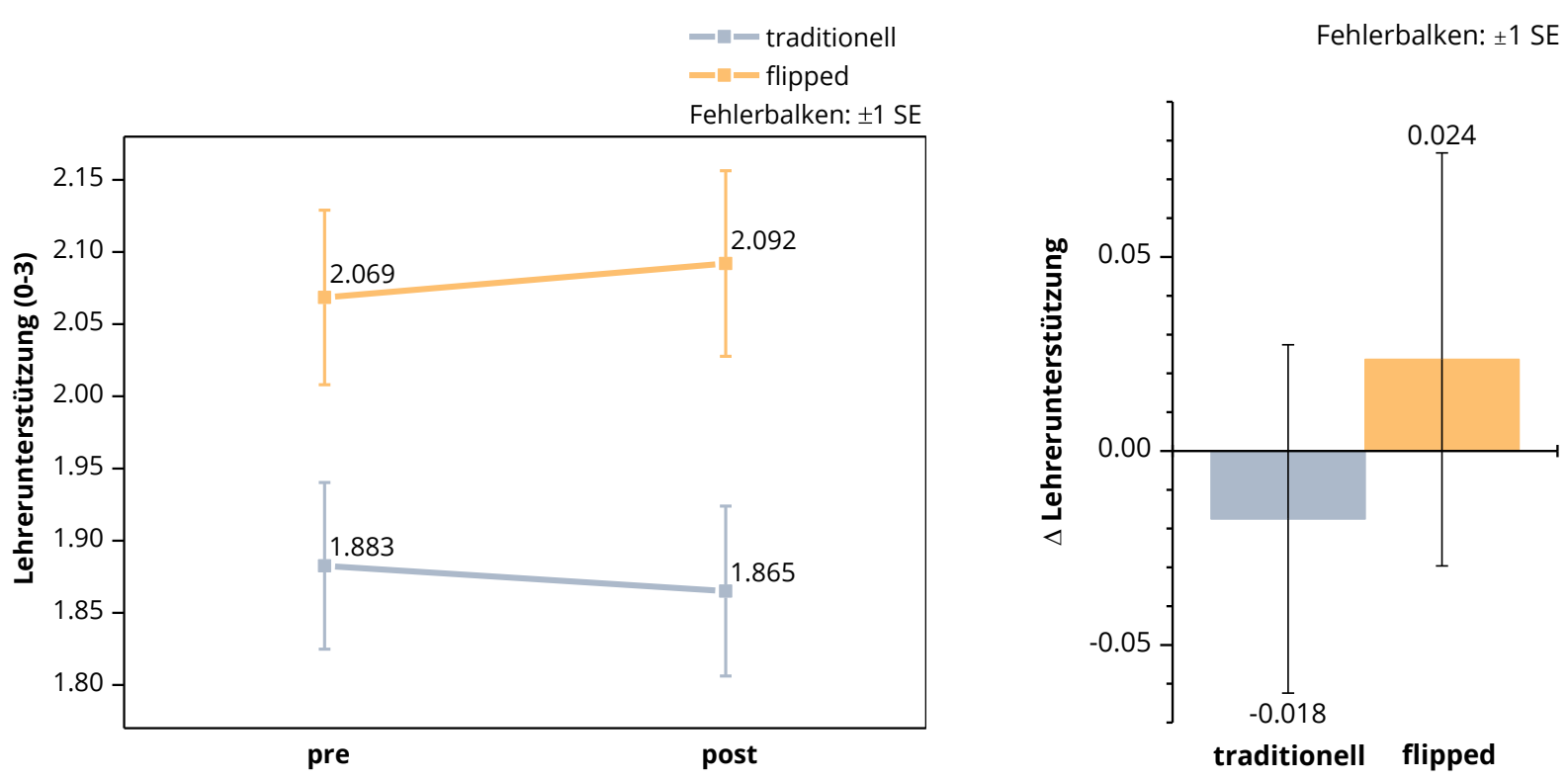

Abb. 26. Entwicklung der Lehrerunterstützung

nicht besser oder schlechter vom Lehrer unterstützt als im traditionellen Unterricht, den sie vorher erlebt haben, obschon die Rolle des Lehrers im Flipped Classroom deutlich anders sein sollte. Die Hypothese $\mathrm{H} 3 \mathrm{a}$, nach der die wahrgenommene Lehrerunterstützung im Flipped Classroom höher ist als im traditionellen Unterricht, muss abgelehnt werden.

\subsubsection{Hypothese 3b: Hausaufgabendisziplin und -dauer}

H3b. Bei vergleichbarem Workload investieren die Schüler im Flipped Classroom durchschnittlich nicht mehr Zeit in die häusliche Vorbereitung für eine Unterrichtsstunde, erledigen sie aber häufiger.

Die letzte Forschungshypothese behauptet, dass sich die Hausaufgabendisziplin von Schülern im Flipped Classroom verbessert, die Schüler also regelmäßiger ihre Hausaufgaben bearbeiten, ohne dass sie im Schnitt einen höheren Workload haben. Ob der Workload vergleichbar ist, ist eine Frage der Planung. Die Schüler des Flipped Classroom sollen in der Basisform nicht mehr Zeit für die häusliche Vorbereitung aufwenden als Schüler im traditionellen For- 
mat für die klassischen Hausaufgaben. Ob das gelungen ist, klärt ein Vergleich der Hausaufgabendauer. Sie wird als direktes Maß für den Workload verstanden. ${ }^{16}$ Die Hausaufgabendisziplin ist Facette des Konstrukts Arbeitshaltung, für das es einen deutlichen Geschlechtereffekt gab. Deshalb soll die Hausaufgabendiszplin zusätzlich nach Jungen und Mädchen differenziert betrachtet werden. Die beiden Variablen Hausaufgabendisziplin und -dauer sind, wie in Kap. 5.4.2 dargestellt, Einzelitems, die sich jedoch intervallskaliert interpretiert lassen.

Hausaufgabendisziplin: Der Levine-Test für den Gain Score $\Delta$ Hausaufgabendisziplin ist signifikant ( $p=.020)$. Die Varianzen sind also nur eingeschränkt homogen. Aber da die Gruppengrößen nicht allzu stark voneinander abweichen, kann die Verletzung der Varianzenhomogenität toleriert werden. Die Schüler der Treatmentgruppe hatten zum Pretestzeitpunkt durchschnittlich eine größere Hausaufgabendisziplin, allerdings war dieser Unterschied nicht signifikant (vgl. Kap. 6.1.2). Die Veränderung der mittleren Hausaufgabendisziplin beider Gruppen zwischen Pre- und Posttest ist in Tab. 26 und illustrativ in Abb. 27 dargestellt.

Tab. 26. Vergleich der Änderungen in der Hausaufgabendisziplin in Kontroll- und Treatmentgruppe

\begin{tabular}{|c|c|c|c|c|c|c|c|c|c|}
\hline & \multicolumn{3}{|c|}{ traditionell } & \multicolumn{3}{|c|}{ flipped } & & & \\
\hline & $N$ & $M$ & $S D$ & $N$ & $M$ & $S D$ & $F$ & $p$ & $\eta p^{2}$ \\
\hline$\triangle H A-D i s z i p l i n$ & 79 & .18 & .73 & 69 & .63 & .84 & 12.700 & $<.001$ & .080 \\
\hline
\end{tabular}
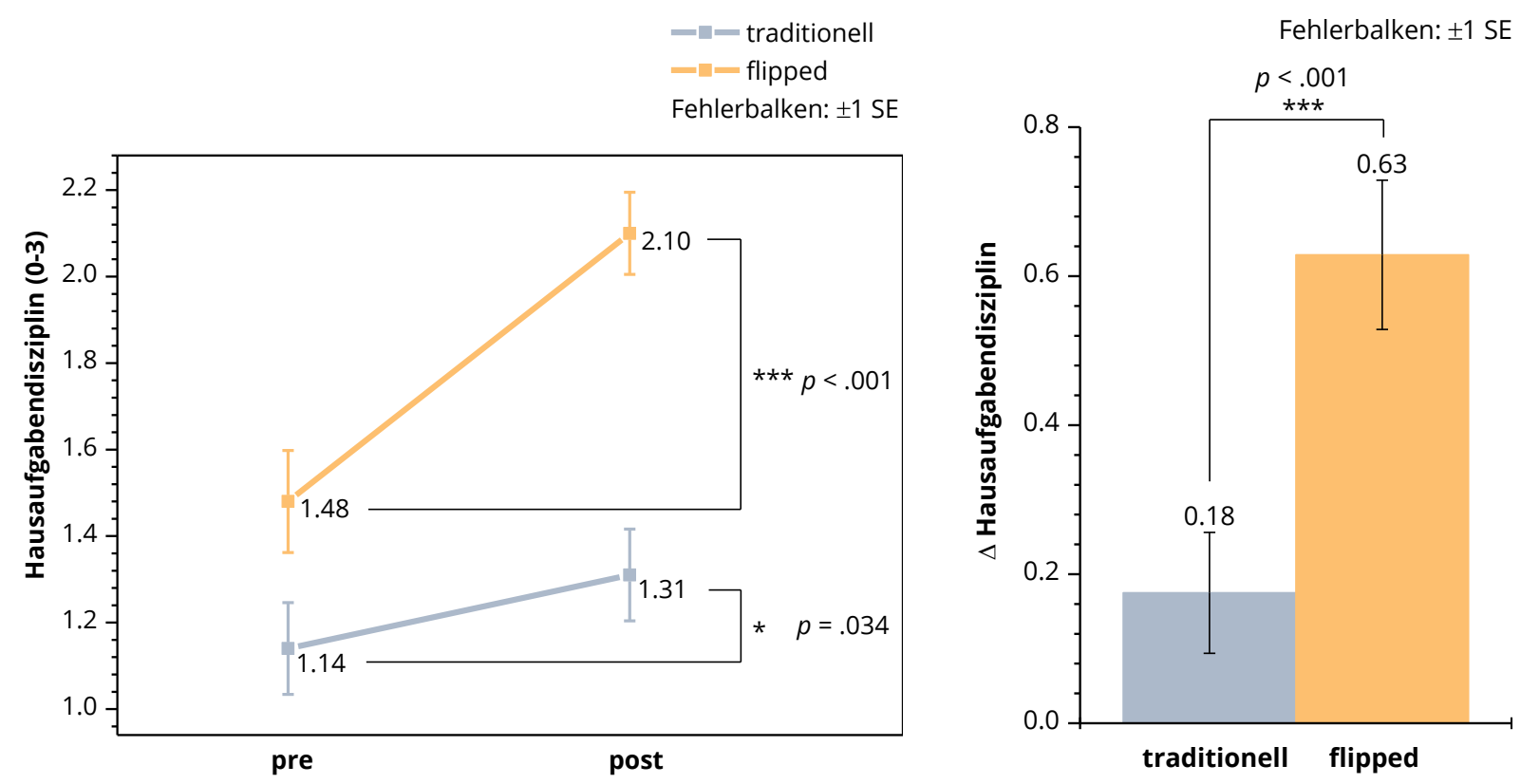

Abb. 27. Änderung der Hausaufgabendisziplin

16 Wie bereits erwähnt, bezieht sich die Hausaufgabendauer auf die Zeit, die durchschnittlich als Nach- oder Vorbereitung für eine Unterrichts(doppel)stunde verwendet wird, nicht auf die Zeit der in der Studie insgesamt investierten häuslichen Arbeit. Bei höherer Hausaufgabendisziplin und konstanter Hausaufgabenzeit erhöht sich selbstverständlich rechnerisch die Gesamtzeit häuslicher Tätigkeit. 
In beiden Gruppen erhöhte sich die Hausaufgabendisziplin im Laufe der Unterrichtsreihe signifikant. Die Schüler aller Kurse erledigten ihre Hausaufgaben also unabhängig von der Methode gewissenhafter als zuvor. Der Zuwachs an Hausaufgabendiszplin ist jedoch im Flipped Classroom deutlich größer als im traditionellen Unterricht. Die Ergebnisse einer ANOVA in Tab. 26 zeigen, dass der Unterschied im Zuwachs an Hausaufgabendiszplin signifikant ist. Der Effekt liegt mit $\eta_{p}{ }^{2}=.08$ im mittleren Bereich.

Hausaufgabendauer: Der Levene-Test für den Gain Score $\Delta$ Hausaufgabendisziplin fällt nicht signifikant aus ( $p=.259)$, Varianzenhomogenität kann vorausgesetzt werden. Wie auch schon bei der Hausaufgabendisziplin verändert sich die Hausaufgabendauer in beiden Gruppen während der Unterrichtsreihe hin zu größeren Werten, wie Tab. 27 und Abb. 28 zeigen.

In beiden Unterrichtsformaten investierten die Schüler mehr Zeit für die Hausaufgaben. Vergleicht man Kontroll- und Treatmentgruppe miteinander, gibt es jedoch keinen signifikanten Unterschied darin, wie sich die durchschnittliche Hausaufgabendauer entwickelt. Zwar verlängert sich die durchschnittliche Hausaufgabendauer innerhalb der Treatmentgruppe signifikant, doch gibt es keinen signifikanten Effekt, wenn man diese Entwicklung mit der Kontrollgruppe vergleicht.

Tab. 27. Vergleich der Entwicklung in der Hausaufgabendauer in Kontroll- und Treatmentgruppe

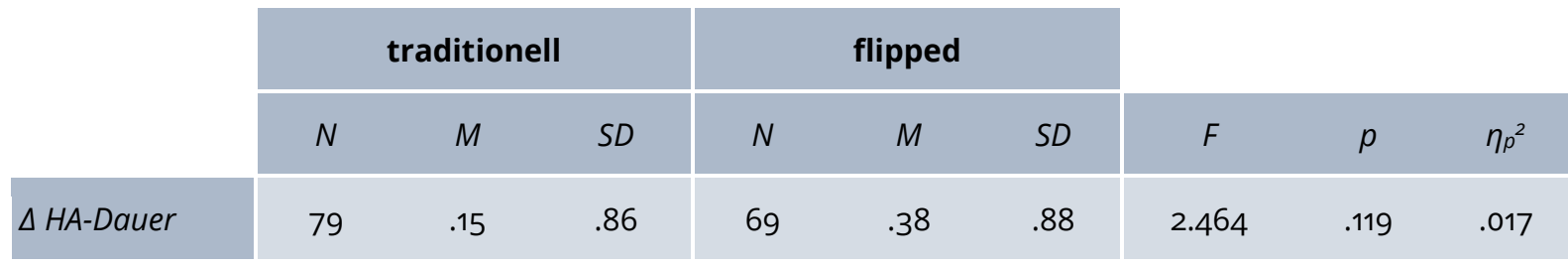
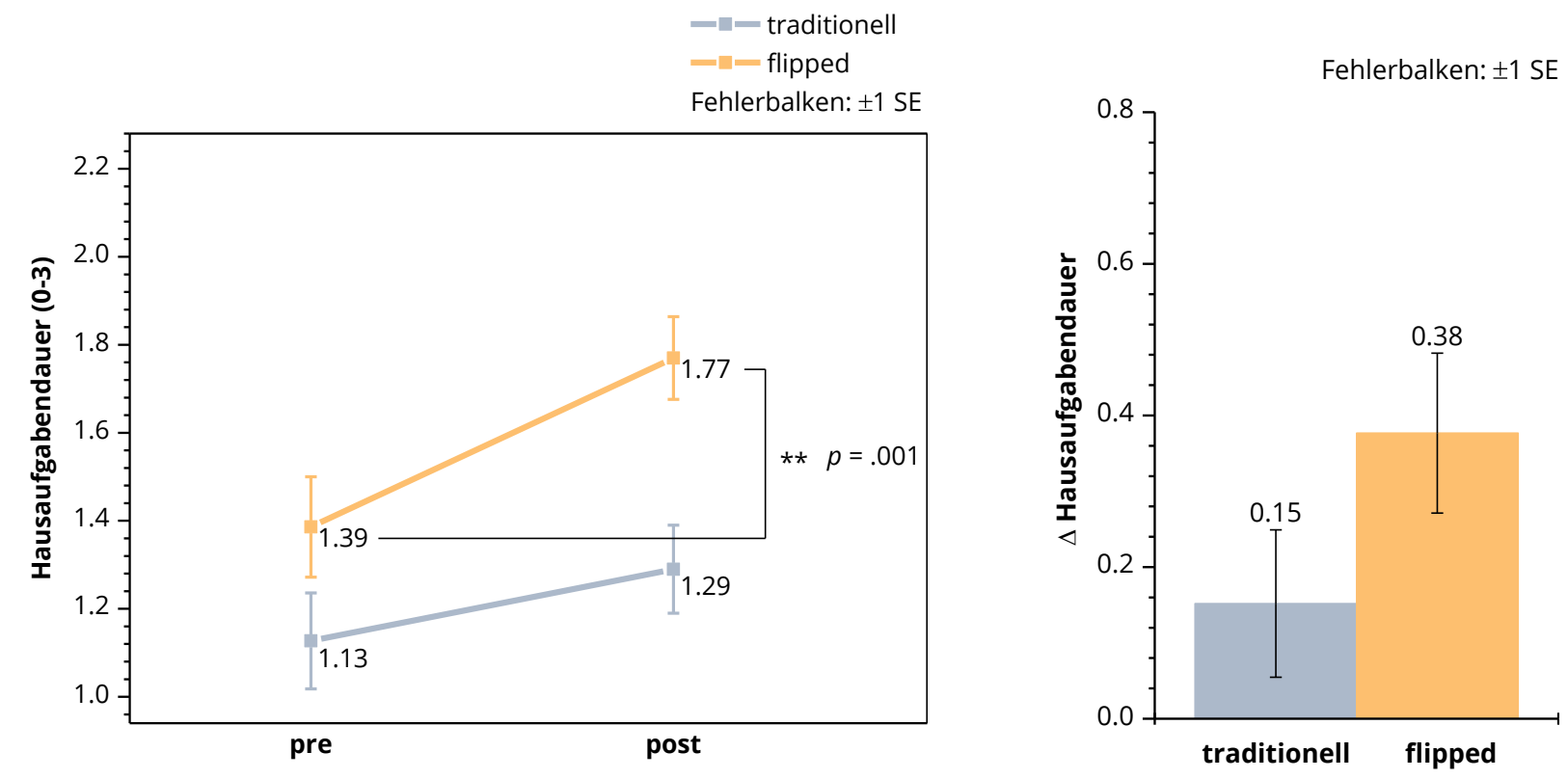

Abb. 28. Änderung der Hausaufgabendauer 
Tab. 28. Mittlere Hausaufgabendauer pre/post in Kontroll- und Treatmentgruppe in Minuten

\begin{tabular}{|c|c|c|c|c|}
\hline & \multicolumn{2}{|c|}{ traditionell } & \multicolumn{2}{|c|}{ flipped } \\
\hline & $M$ & $S D$ & $M$ & $S D$ \\
\hline HA-Dauer pre & $8.45 \mathrm{~min}$ & $5.55 \min$ & $10.11 \mathrm{~min}$ & $5.85 \mathrm{~min}$ \\
\hline HA-Dauer post & $9.21 \mathrm{~min}$ & $5.18 \mathrm{~min}$ & $12.28 \mathrm{~min}$ & $5.45 \mathrm{~min}$ \\
\hline$\triangle H A-D a u e r$ & $.76 \mathrm{~min}$ & $5.07 \mathrm{~min}$ & $2.17 \mathrm{~min}$ & $6.03 \mathrm{~min}$ \\
\hline
\end{tabular}

Um die Ergebnisse zur durchschnittlichen Hausaufgabendauer etwas anschaulicher werden zu lassen, sind in Tab. 28 die nominalen Angaben der Schüler - wie in Kap. 6.1.2 - näherungsweise in Minuten umgerechnet $(\mathrm{o}=2.5 \mathrm{~min}, 1=7.5 \mathrm{~min}, 2=12.5 \mathrm{~min}, 3=22.5 \mathrm{~min})$. Genähert in Minuten bedeutet es, dass die Schüler im Flipped Classroom im Mittel etwa 2 Minuten länger in ihre häusliche Vorbereitung investierten, als sie es zuvor im traditionellen Unterricht getan hatten. Wenn man berücksichtigt, dass parallel auch die Schüler in der Kontrollgruppe im Schnitt eine dreiviertel Minute länger an den Hausaufgaben arbeiteten als zuvor, nivelliert sich dieser Unterschied nahezu vollständig.

Geschlecht: Abschließend soll noch ein Blick auf die Entwicklung der Hausaufgabendisziplin und -dauer bei Jungen und Mädchen geworfen werden, da die Lernausgangslage (vgl. Tab. 17) zeigte, dass es in diesen Variablen einen deutlichen Unterschied zwischen Jungen und Mädchen gab. Die Mädchen hatten zum Prezeitpunkt signifikant höhere Werte in beiden Größen.

Vergleicht man die Veränderungen in beiden Hausaufgabengrößen (vgl. Tab. 29), gibt es innerhalb der Gruppe der Mädchen keine signifikanten Unterschiede zwischen dem traditionellen Unterricht und Flipped Classroom. Die Hausaufgabendisziplin ändert sich bei den Mädchen praktisch nicht. Bei den Jungen hingegen ist - im Vergleich zum traditionellen Unterricht - eine deutliche Verbesserung der Hausaufgabendisziplin im Flipped Classroom festzustellen. In Abb. 29 werden diese Daten grafisch veranschaulicht.

Tab. 29. Entwicklung der Hausaufgabenparameter differenziert nach Mädchen und fungen in Kontroll- und Treatmentgruppe

\begin{tabular}{|c|c|c|c|c|c|c|c|c|c|}
\hline & \multicolumn{3}{|c|}{ traditionell } & \multicolumn{3}{|c|}{ flipped } & \multirow[b]{2}{*}{$F$} & \multirow[b]{2}{*}{$p$} & \multirow[b]{2}{*}{$\eta_{p^{2}}$} \\
\hline & $N$ & $M$ & $S D$ & $N$ & $M$ & $S D$ & & & \\
\hline Mädchen ( $\triangle$ HA-Disziplin) & 17 & .00 & .71 & 23 & .30 & .88 & 1.383 & .247 & .035 \\
\hline Mädchen ( $\triangle$ HA-Dauer) & 17 & .06 & 1.03 & 23 & .26 & 1.10 & .035 & .558 & .009 \\
\hline Jungen ( $\triangle H A$-Disziplin) & 63 & .22 & .73 & 47 & .79 & .78 & 15.274 & $<.001$ & .124 \\
\hline Jungen ( $\triangle H A-D a u e r)$ & 62 & .18 & .82 & 46 & .43 & .75 & 2.794 & .098 & .026 \\
\hline
\end{tabular}




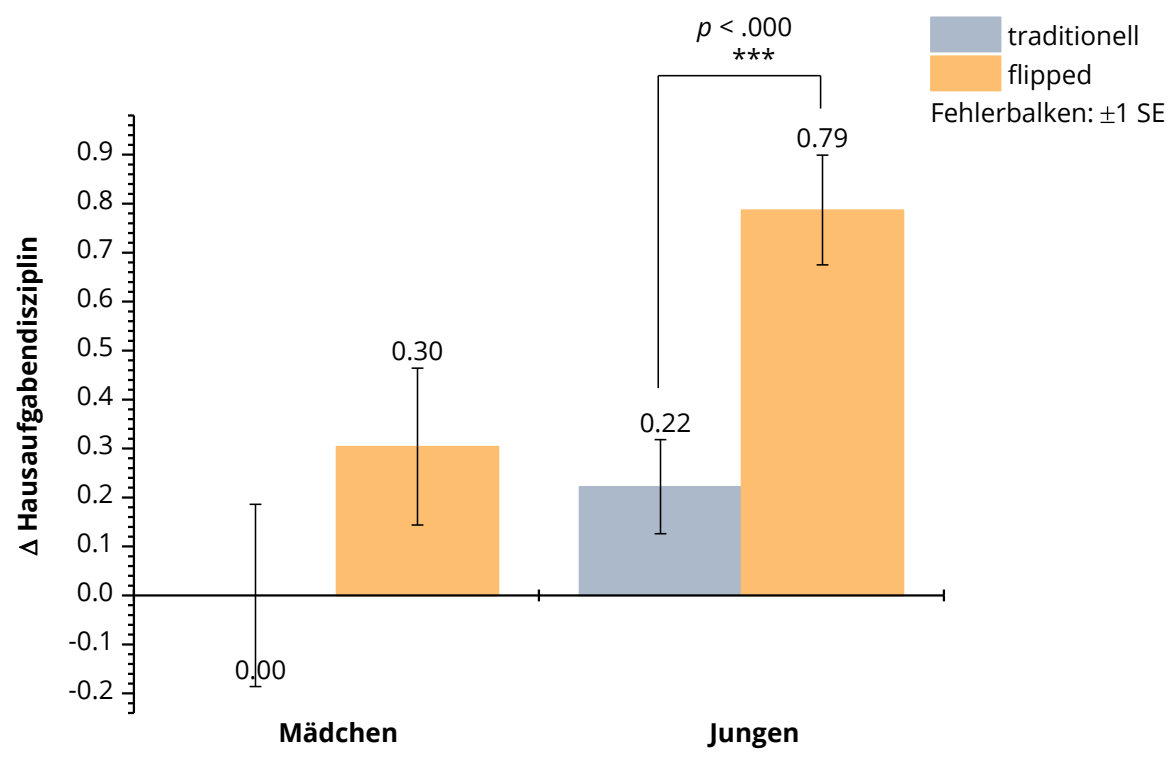

Abb. 29. Änderung der Hausaufgabendisziplin in Kontroll- und Treatmentgruppe differenziert nach Geschlecht

Aufgrund der Tatsache, dass sich die Hausaufgabendisziplin auch bei den Mädchen im Flipped Classroom etwas verbessert - wenn auch nicht signifikant -, gibt es aber keinen Geschlechtereffekt im Flipped Classroom. Eine ANOVA mit den unabhängigen Faktoren $\mathrm{Me}$ thode und Geschlecht zeigt keine signifikante Interaktion der beiden Größen, $F(1,146)=.253$, $p=.616$. Nimmt man also die Mädchen vergleichend hinzu, kann man nicht sagen, dass die Jungen im Flipped Classroom ihre Hausaufgabendisziplin stärker verbessern als die Mädchen. Vergleicht man sie aber mit ihren männlichen Mitschülern der Kontrollgruppe, ist die Verbesserung in der Hausaufgabendiszplin signifikant, $F(1,108)=15.274, p<.001, \eta_{p}{ }^{2}=.124$. Daher lässt sich begründet sagen, dass es hauptsächlich die Jungen sind, die im Flipped Classroom deutlich regelmäßiger die Lernvideos zur Unterrichtsvorbereitung anschauen als im traditionellen Unterricht die schriftlichen Hausaufgaben zu bearbeiten. Von allen Facetten der $A r-$ beitshaltung sind nur die hausaufgabenbezogenen Variablen zum Pre- und Postzeitpunkt erhoben worden. Deshalb lässt sich auch nur auf diese Variablen ein Effekt des Flipped Classroom messen. Da aber die Arbeitshaltung der Mädchen zum Prezeitpunkt in allen Facetten deutlich besser ist als die der Jungen, ist die signifikante Verbesserung der Hausaufgabendisziplin bei den Jungen durch den Flipped Classroom ein beachtenswerter Befund.

\subsubsection{Einfluss der Lehrkraft}

Abschließend soll auch für die Forschungsfrage 3 untersucht werden, ob die unterrichtende Lehrkraft einen Einfluss auf die Lehrerunterstützung und die Stützfaktoren hatte, auch wenn angesichts der kleinen Stichprobenzahl von teilnehmenden Lehrkräften eine solche Untersuchung nicht belastbar ist. Da es der Lehrer ist, der die Schüler im Unterricht im Lernprozess unterstützt und ihnen häusliche Arbeit zur Vor- oder Nachbereitung erteilt, soll nun der Frage nachgegangen werden, ob es eine Interaktion von Lehrer und Methode gibt, es also möglicherweise von der Kombination von Lehrer und Methode abhängt, wie sich Lehrerunterstützung 
und die Stützfaktoren ändern. Dazu muss - wie in Kap. 6.3.3 - die Stichprobe auf die Kurse reduziert werden, die von den Lehrern unterrichtet wurden, die beide Unterrichtsformen unterrichtet haben, also die Lehrer 1-3.

Der Levene-Test ist für keine der Variablen signifikant. In Tab. 30 sind die ANOVA-Ergebnisse der Interaktionen von Lehrer und Methode aufgeführt. Der Effekt der Methode auf die Hausaufgabendisziplin besteht auch in dieser Berechnung als Haupteffekt weiterhin. Auf die Hausaufgabendauer sowie die Lehrerunterstützung hat die Methode, wie oben gesehen, keinen Effekt.

Es zeigt sich zunächst, dass es keine Interaktion von Methode und Lehrer hinsichtlich der Änderung der Lehrerunterstützung gibt. Zusammen mit dem Befund von Kap. 6.4.1 bedeutet das, dass weder die Methode, noch die Kombination bestimmter Lehrer mit der Methode etwas an der Art änderten, wie sich die Schüler unterstützt fühlten. In keinem der drei Kurse der Treatmentgruppe hat sich die wahrgenommene Lehrerunterstützung signifikant zu den anderen geändert. Für die Schüler machte weder der Flipped Classroom noch die Tatsache, von einem bestimmten Lehrer im Flipped Classroom unterrichtet zu werden, einen Unterschied. Der Wert der wahrgenommenen Lehrerunterstützung zum Postzeitpunkt war nicht signifikant anders als zum Prezeitpunkt.

Hingegen wird der Effekt des Flipped Classroom auf den Stützfaktor Hausaufgabendisziplin sehr wohl durch den Faktor Lehrer moderiert. Hier kam es also auf die Kombination von Methode und Lehrer an, wie sich die Stützfaktoren entwickelt haben. Neben dem in Kap. 6.4.2 dargestellten Haupteffekt der Methode auf die Hausaufgabendisziplin gibt es einen Nebeneffekt, der durch die Interaktion hervorgerufen wird, $F_{M e t h}{ }^{*}$ Lehrer $(2,212)=5.183, p=.007$, $\eta_{p}{ }^{2}=.085$, was einem mittleren Effekt entspricht. Das bedeutet, dass es für die Hausaufgabendisziplin der Schüler wichtig war, welcher Lehrer Flipped Classroom unterrichtete. In manchen Kursen der Treatmentgruppe entwickelte sich die Hausaufgabendisziplin anders als in anderen.

In Kap. 6.4.2 wurde dargestellt, dass die Methode keinen Einfluss auf die Entwicklung der Hausaufgabendauer hatte. Nimmt man jedoch den Lehrer als festen Faktor hinzu, zeigt sich eine signifikante Interaktion von Lehrer und Methode, $F(2,212)=3.292, p=.041, \eta_{p}{ }^{2}=.056$, was einem kleinen Effekt entspricht. Ob sich also die Hausaufgabendauer der Schüler änderte, hing - ähnlich wie bei der Hausaufgabendisziplin - davon ab, welchen Lehrer sie im Flipped Classroom als Kursleiter hatten.

Tab. 3o. Einfluss der Unterrichtsmethode und der Lehrkraft auf die Änderung der Lehrerunterstützung und der Stützfaktoren

\begin{tabular}{l|ccccc|} 
& $d f$ & $d f$ Residuen & $F_{\text {Meth*Lhrer }}$ & $p$ & $\eta_{p^{2}}$ \\
\hline$\Delta$ Lehrerunterstützung & 2 & 112 & 1.455 & .238 & .025 \\
\hline$\Delta$ HA-Disziplin & 2 & 112 & $\mathbf{5 . 1 8 3}$ & .007 & .085 \\
\hline$\Delta$ HA-Dauer & 2 & 112 & $\mathbf{3 . 2 9 2}$ & .041 & .056
\end{tabular}




\subsubsection{Diskussion}

Lehrerunterstützung: Im Flipped Classroom fühlten sich die Schüler nicht stärker von ihren Lehrern unterstützt als zuvor. Die wahrgenommene Lehrerunterstützung änderte sich im Flipped Classroom nicht, sondern blieb - wie auch in der Kontrollgruppe - praktisch auf dem Niveau zum Prezeitpunkt. Ergänzend ließ sich feststellen, dass es dabei auch keine Rolle spielte, welcher Lehrer im Flipped Classroom unterrichtete - in keinem Flipped ClassroomKurs hatte sich die Lehrerunterstützung signifikant gegenüber anderen Kursen oder gegenüber den Kontrollgruppenkursen geändert. Dieser Befund ist überraschend. Zum einen ist die konzeptionelle Anlage des Flipped Classroom unter anderem genau darauf ausgerichtet, die Schüler besser individuell begleiten zu können (vgl. Kap. 2.3.3), und zum anderen zeigt eine breite Forschungslage, dass Lernende vor allem die stärkere Unterstützung und Beratung durch den Lehrer im Flipped Classroom schätzen (Butt 2014; Greenberg et al. 2011; Hao 2016; Schultz et al. 2014), auch wenn es dazu Ausnahmen gibt (Sun \& Wu 2016; Van Sickle 2016). Wie lässt sich diese Diskrepanz zum Befund der vorliegenden Studie erklären?

Offenbar machte es für die Schüler subjektiv keinen Unterschied, ob der Lehrer jemand war, der im traditionellen, darbietenden und am Experiment orientierten Physikunterricht durch fragend-entwickelnde Gesprächsführung bemüht war, die Schüler zu aktivieren, oder ein eher adaptiver Lernbegleiter, den sie im Flipped Classroom in der Partner- oder Gruppenarbeit nur wahrnahmen, wenn er aufmerksam neben ihnen stand oder mit ihnen durch Hilfestellungen, Fragen und Anregungen interagierte. Der Flipped Classroom vermochte dem Physikunterricht der teilnehmenden Lehrkräfte keine zusätzliche Qualität an Schülerbegleitung zu geben. Das bedeutet, dass die Lehrer entweder resistent gegenüber dem methodisch geforderten Rollenwechsel im Flipped Classroom waren oder - wovon eher auszugehen ist dass sie von ihren Schülern schon zuvor als unterstützend und lernbegleitend wahrgenommen wurden. Wenn es in der subjektiv empfundenen Lehrerunterstützung zwischen traditionellem Unterricht und Flipped Classroom keinen signifikanten Unterschied gibt, deutet das nicht unbedingt auf ein Defizit der Methode oder ihrer Umsetzung hin, sondern eher auf einen hohen Grad an Lehrer-Schüler-Interaktion im traditionellen Unterricht der betreffenden Lehrer. Diese Interpretation des Befunds wird gestützt durch die Feststellung, dass sämtliche oben genannten Studien, bei denen eine deutliche Veränderung in der Lehrerunterstützung durch Flipped Classroom gemessen wurde, im Hochschulbereich durchgeführt wurden (Butt 2014; Greenberg et al. 2011; Hao 2016; Schultz et al. 2014). Diese Lernumgebungen lassen im traditionellen Charakter der Vorlesung viel weniger Interaktionen zwischen Dozent und Student zu, als das im Unterrichtsgeschehen allgemeinbildender Schulen möglich ist. Flipped Classroom kann möglicherweise besonders in jenen Bildungssystemen Veränderung der Lehrerunterstützung bewirken, in denen das Vergleichsformat eine traditionelle Vorlesung ist. Es ist eine naheliegende Erklärung für den zunächst überraschenden Widerspruch zu Hypothese H3a, dass sich der Flipped Classroom nicht auf die Lehrerunterstützung auswirkte, weil diese schon auf einem Niveau lag, das sich durch Flipped Classroom nicht mehr signifikant steigern ließ. 
Hausaufgabendisziplin und -dauer: Die Schüler im Flipped Classroom haben im Schnitt nicht mehr Zeit für ihre häusliche Vorbereitung auf eine Unterrichtsstunde benötigt als ihre Mitschüler in der Kontrollgruppe. Allerdings haben sie ihre vorbereitenden Aufgaben wesentlich regelmäßiger bearbeitet als die Schüler im traditionellen Unterricht. Ihre Hausaufgabendisziplin ist signifikant besser geworden, während die durchschnittliche Hausaufgabendauer konstant geblieben ist. Hypothese $\mathrm{H}_{3} \mathrm{~b}$ kann damit angenommen werden, unbeschadet der Tatsache, dass bei gleichbleibender Hausaufgabendauer und verbesserter Hausaufgabendisziplin die effektive Gesamtzeit an häuslicher Vorbereitung selbstverständlich zugenommen hat.

Die Daten zeigen, dass die Hausaufgabendisziplin auch in der Kontrollgruppe während der Studie angestiegen ist (vgl. Abb. 27). Zwar ist der Anstieg im Flipped Classroom deutlich steiler, doch auch in der Kontrollgruppe ist die Zunahme an Hausaufgabendisziplin signifikant. Ein Grund dafür könnte sein, dass der Zeitraum der Unterrichtsreihe im zweiten Halbjahr lag, auf das Ende des Schuljahres und damit auf abiturrelevante Zeugnisnoten zulief. Womöglich waren die Schüler unabhängig von der eingesetzten Unterrichtsmethode zunehmend motiviert, ihre Hausaufgaben regelmäßiger anzufertigen. Das mag besonders auf die Jungen zutreffen, denn vor allem sie steigerten ihre Hausaufgabendisziplin in beiden Unterrichtsformaten (vgl. Abb. 29). Innerhalb der Treatmentgruppe unterscheiden sich Mädchen und Jungen nicht in der Entwicklung ihrer Hausaufgabendisziplin und -dauer voneinander. Verglichen mit den Jungen im traditionellen Unterricht jedoch verbesserten die Jungen im Flipped Classroom ihre Hausaufgabendisziplin deutlich. Den Jungen schien das Aufgabenformat, sich zuhause mit den Lernvideos auf den Unterricht vorzubereiten, deutlich mehr entgegenzukommen als das klassische Format, zuhause schriftliche Hausaufgaben anzufertigen. Die Hausaufgabendisziplin ist ein Teilaspekt der Arbeitshaltung. Dieser Aspekt konnte im Flipped Classroom eindeutig gefördert werden, vor allem bei den Jungen, die im Vergleich zu den Mädchen wesentlich schlechtere Voraussetzungen in der Arbeitshaltung aufgewiesen hatten.

Die Entwicklung der Hausaufgabendauer verläuft teilweise parallel zu der der Hausaufgabendisziplin. Unabhängig von der Methode stieg in beiden Gruppen die mittlere Hausaufgabendauer an, im Flipped Classroom zwar signifikant zum Prezeitpunkt, aber nicht signifikant zur Kontrollgruppe (vgl. Abb. 28). Auch hier dient der Zeitpunkt der Studie im zweiten Halbjahr als mutmaßliche Erklärung für die Entwicklung in beiden Gruppen. Durch den Wunsch, das Schuljahr mit persönlich zufriedenstellenden Leistungen abzuschließen, waren die Schüler möglicherweise stärker extrinsisch motiviert, ihre Hausaufgaben sowohl regelmäßiger als auch ausdauernder zu bearbeiten. Es war jedoch nicht Teil der vorliegenden Studie, diesen Wirkzusammenhang zu untersuchen. Tatsache ist, dass alle Schüler am Ende der Studie durchschnittlich mehr Zeit für ihre Hausaufgaben investierten als vorher, wodurch die erhöhte Hausaufgabendauer im Flipped Classroom nicht mehr signifikant ist. Um die Wirkung des Flipped Classroom nicht vorschnell einem erhöhten Pensum an häuslicher Arbeit zuzuschreiben, war es für das Design der Studie wichtig, vergleichbare Bedingungen zu schaffen. 
Dadurch, dass sich die Entwicklungen der Hausaufgabendauer in Kontroll- und Treatmentgruppe nicht unterscheiden, kann man von vergleichbarem häuslichen Workload ausgehen, was der Validität der Studie zugutekommt.

Es ist anzumerken, dass die Ergebnisse zur Hausaufgabendisziplin und -dauer relativiert werden müssen, wenn man zusätzlich den Faktor Lehrer berücksichtigt. Zwar ist das mit lediglich drei zu berücksichtigenden Lehrern nur ansatzweise möglich, doch zeigt sich bereits hier, dass die Entwicklung der Stützfaktoren Hausaufgabendisziplin und -dauer auch davon abhing, welcher Lehrer im Flipped Classroom unterrichtete. Zwar lässt sich pauschal zunächst sagen, dass der Flipped Classroom die Hausaufgabendisziplin der Schüler verbesserte, doch machte es zusätzlich einen Unterschied, welcher Lehrer Flipped Classroom unterrichtete. Für die Entwicklung der Hausaufgabendauer gibt es - wie oben bereits diskutiert - keinen Haupteffekt, sondern ausschließlich einen Interaktionseffekt. Bei bestimmten Lehrern verkürzte sich die Zeit der häuslichen Vorbereitung durch Flipped Classroom, bei anderen verlängerte sie sich. Dieser Befund überrascht nicht, da der Umfang der Hausaufgaben grundsätzlich immer im Ermessen des einzelnen Lehrers liegt und nur durch die Intervention mit den Lernvideos für alle Kurse auf einheitliches Maß normalisiert wurde. Im Mittel hat sich die Zeit der häuslichen Vorbereitung für die Schüler durch den Flipped Classroom nicht signifikant geändert. Wenn man den einzelnen Lehrer mit in Betracht zieht, lässt sich diese Aussage pauschal nicht halten. Hier bedarf es einer größeren Zahl teilnehmender Lehrkräfte, um zuverlässigere Ergebnisse in dieser Frage zu erzielen.

Eine weitere Überlegung ist noch zu berücksichtigen: Die Kombination von Methode und Lehrer ist gleichbedeutend mit dem Einzelfaktor Kurs, denn jeder Lehrer unterrichtete pro Methode genau einen Physikkurs. Deshalb lässt sich in diesem Studiendesign nicht unterscheiden, ob es tatsächlich die Interaktion von Methode und individuellem Lehrer war, die für eine unterschiedliche Entwicklung der Hausaufgabendisziplin und -dauer verantwortlich war, oder eine kursspezifische Gruppenkonstellation und -dynamik. Auch hier bedarf es einer größeren Anzahl von Lehrern und Kursen, um in einer Mehrebenenanalyse Klarheit über die Interaktionseffekte zu erhalten.

\subsection{Rückmeldungen der Schüler im Flipped Classroom}

Die bisherigen Befunde sollen nun ergänzt werden durch Rückmeldungen der Schüler zum Flipped Classroom. Im Posttest hatten die Schüler der Treatmentgruppe Gelegenheit zu einem Feedback sowohl in geschlossener Fragebogenform als auch in offener, stichpunktartiger Form. Dieses Unterkapitel ist strukturiert nach den charakteristischen Elementen der Flipped Classrooms - den Lernvideos, den Übungsaufgaben, den Experimenten und der veränderten Lehrerrolle. Zunächst werden die Ergebnisse des Fragebogens in Tab. 31 und Tab. 32 in Gänze dargestellt, bevor sie in den einzelnen Unterkapiteln analysiert werden. 
Tab. 31. Rückmeldungen der Schüler zum Flipped Classroom (Fragebogen) mit integriertem Säulendiagramm

\begin{tabular}{|c|c|c|c|c|c|}
\hline & & $\begin{array}{l}\text { Stimme } \\
\text { überhaupt } \\
\text { nicht zu }\end{array}$ & $\begin{array}{l}\text { Stimme } \\
\text { eher nicht } \\
\text { zu }\end{array}$ & $\begin{array}{l}\text { Stimme } \\
\text { eher zu }\end{array}$ & $\begin{array}{l}\text { Stimme } \\
\text { völlig zu }\end{array}$ \\
\hline 1. & $\begin{array}{l}\text { Bei dieser Methode musste ich zuhause } \\
\text { mehr tun als im normalen Unterricht. }\end{array}$ & $\begin{array}{c}N=8 \\
(11,4 \%)\end{array}$ & $\begin{array}{l}N=16 \\
(22,9 \%)\end{array}$ & $\begin{array}{l}N=22 \\
(31,4 \%)\end{array}$ & $\begin{array}{l}N=24 \\
(34,3 \%)\end{array}$ \\
\hline 2. & $\begin{array}{l}\text { Ich war mit der Vermittlung der Inhalte } \\
\text { überfordert. }\end{array}$ & $\begin{array}{l}N=18 \\
(25,7 \%)\end{array}$ & $\begin{array}{l}N=35 \\
(50,0 \%)\end{array}$ & $\begin{array}{c}N=16 \\
(22,9 \%)\end{array}$ & $\begin{array}{l}N=1 \\
(1,4 \%)\end{array}$ \\
\hline 3. & $\begin{array}{l}\text { Bei dieser Methode musste ich im Unter- } \\
\text { richt mehr tun als im normalen Unter- } \\
\text { richt. }\end{array}$ & $\begin{array}{l}N=6 \\
(8.6 \%)\end{array}$ & $\begin{array}{l}N=26 \\
(37.1 \%)\end{array}$ & $\begin{array}{l}N=23 \\
(32.9 \%)\end{array}$ & $\begin{array}{l}N=15 \\
(21.4 \%)\end{array}$ \\
\hline 4. & $\begin{array}{l}\text { Bei dieser Methode habe ich aktiver mit } \\
\text { meinen Mitschülern gearbeitet als im } \\
\text { normalen Unterricht. }\end{array}$ & $\begin{array}{l}N=9 \\
(12.9 \%)\end{array}$ & $\begin{array}{l}N=13 \\
(18.6 \%)\end{array}$ & $\begin{array}{l}N=23 \\
(32.9 \%)\end{array}$ & $\begin{array}{l}N=25 \\
(35 \cdot 7 \%)\end{array}$ \\
\hline 5. & $\begin{array}{l}\text { Wenn ich die Experimente live statt nur } \\
\text { im Video gesehen hätte, hätte ich mehr da- } \\
\text { von gehabt. }\end{array}$ & $\begin{array}{l}N=11 \\
(15 \cdot 7 \%)\end{array}$ & $\begin{array}{l}N=25 \\
(35.7 \%)\end{array}$ & $\begin{array}{l}N=16 \\
(22.9 \%)\end{array}$ & $\begin{array}{l}N=18 \\
(25 \cdot 7 \%)\end{array}$ \\
\hline 6. & $\begin{array}{l}\text { Ich glaube, mein Physiklehrer hat bei die- } \\
\text { ser Methode ein besseres Bild davon, was } \\
\text { ich kann und was nicht. }\end{array}$ & $\begin{array}{l}N=16 \\
(22.9 \%)\end{array}$ & $\begin{array}{l}N=25 \\
(35.7 \%)\end{array}$ & $\begin{array}{l}N=22 \\
(31.4 \%)\end{array}$ & $\begin{array}{c}N=7 \\
(10.0 \%)\end{array}$ \\
\hline 7. & $\begin{array}{l}\text { Mein Physiklehrer konnte bei Problemen, } \\
\text { die ich hatte, besser auf mich eingehen. }\end{array}$ & $\begin{array}{l}N=3 \\
(4 \cdot 3 \%)\end{array}$ & $\begin{array}{l}N=28 \\
(40.0 \%)\end{array}$ & $\begin{array}{l}N=29 \\
(41.4 \%)\end{array}$ & $\begin{array}{l}N=10 \\
(14 \cdot 3 \%)\end{array}$ \\
\hline 8. & $\begin{array}{l}\text { Das typische Lehrer-Schüler-Gespräch, } \\
\text { mit dem gemeinsam die Inhalte entwickelt } \\
\text { werden, hat mir bei der Flipped Classroom } \\
\text { Methode gefehlt. }\end{array}$ & $\begin{array}{l}N=5 \\
(7.1 \%)\end{array}$ & $\begin{array}{l}N=20 \\
(28.6 \%)\end{array}$ & $\begin{array}{l}N=25 \\
(35.7 \%)\end{array}$ & $\begin{array}{l}N=20 \\
(28.6 \%)\end{array}$ \\
\hline 9. & $\begin{array}{l}\text { Diese Methode fand ich für acht Wochen } \\
\text { zu lang und zu eintönig. }\end{array}$ & $\begin{array}{l}N=16 \\
(22.9 \%)\end{array}$ & $\begin{array}{l}N=24 \\
(34 \cdot 3 \%)\end{array}$ & $\begin{array}{l}N=21 \\
(30.0 \%)\end{array}$ & $\begin{array}{c}N=9 \\
(12.8 \%)\end{array}$ \\
\hline 10. & $\begin{array}{l}\text { Mit der Flipped Classroom Methode habe } \\
\text { ich mehr gelernt im Vergleich zum norma- } \\
\text { len Unterricht. }\end{array}$ & $\begin{array}{l}N=13 \\
(18.6 \%)\end{array}$ & $\begin{array}{l}N=25 \\
(35.7 \%)\end{array}$ & $\begin{array}{l}N=21 \\
(30.0 \%)\end{array}$ & $\begin{array}{l}N=11 \\
(15 \cdot 7 \%)\end{array}$ \\
\hline
\end{tabular}

Anmerkung: $N_{\text {flip }}=70$

Tab. 32. Veränderung der Screen Time der Schüler (Zeit vor einem Bildschirm)

\footnotetext{
Wie sehr hat der Einsatz der Videos die Zeit verändert, die Sie insgesamt vor einem Bildschirm verbracht haben?
}

\begin{tabular}{ccc}
$\begin{array}{c}\text { Die Gesamtzeit } \\
\text { wurde nicht } \\
\text { wirklich länger. }\end{array}$ & $\begin{array}{c}\text { Die Gesamtzeit } \\
\text { wurde ein } \\
\text { wenig länger. }\end{array}$ & $\begin{array}{c}\text { Die Gesamtzeit } \\
\text { wurde deutlich } \\
\text { länger. }\end{array}$ \\
\hline $\mathrm{N}=38$ & $\mathrm{~N}=29$ & $\mathrm{~N}=3$ \\
$(54.3 \%)$ & $(41.4 \%)$ & $(4.3 \%)$ \\
\hline
\end{tabular}

Anmerkung: $N_{\text {flip }}=70$ 


\subsubsection{Lernvideos und häusliche Vorbereitungszeit}

$\mathrm{Zu}$ Beginn jeder Unterrichtsstunde gaben die Schüler beim Clicker-Quiz im Flipped Classroom anonym mittels der Clicker an, ob und in welchem Umfang sie das Lernvideo zu Hause angesehen hatten. Gemittelt über alle 16 Videos ergeben sich die in Tab. 33 dargestellten Werten.

Diese Angaben fügen sich in das Bild ein, das sich aus der Hausaufgabendisziplin zum Postzeitpunkt ergibt (Item: »Wie regelmäßig haben Sie in den letzten zwei Monaten die Physikhausaufgaben erledigt? « Selten/nie: 14.0\%, Hin und wieder: 25.3\%, Häufig: 39.7\%, Immer: 20.5\%), allerdings setzen die beiden Angaben unterschiedliche Schwerpunkte. Die Hausaufgabendisziplin ist eine Selbsteinschätzung der Gewissenhaftigkeit, während die Clickerfrage nach dem Umfang fragt, in dem ein Lernvideo betrachtet wurde. Gemäß dieser anonymen Rückmeldung haben durchschnittlich etwa $10 \%$ der Schüler das Lernvideo für die nächste Unterrichtsstunde nicht gesehen, weitere 14\% nur zum Teil. Etwa 75\% der Schüler haben demnach das Video, das die Unterrichtsstunde vorbereitet, mindestens einmal angesehen. Das entspricht etwas mehr als dem Wert von 68\%, den Bell (2015) in einer Studie zum Flipped Classroom in einem US-amerikanischen High School-Physikkurs erhoben hat.

Die Nutzungsstatistik für die Lernvideos in Abb. 30 zeigt teilweise deutliche Schwankungen in der Nutzung der einzelnen Videos. Während etwa Video 4 überdurchschnittlich gründlich gesehen wurde, sind Video 7 und 10 von vielen Schülern nicht vollständig geschaut worden. Das erschwert in der Folge das Verständnis der beiden Videos, wie weiter unten zu sehen ist. Ansonsten fallen keine übermäßigen Schwankungen oder strukturellen Veränderungen im Nutzungsverhalten während der Studie auf.

Für die Kontrollgruppe wurde kein vergleichbares Hausaufgaben-Monitoring durchgeführt, so dass in dieser Studie nicht nachvollzogen werden kann, welcher Anteil der Schüler im traditionellen Unterricht in welchem Umfang die Hausaufgaben angefertigt hat. Das enge Monitoring im Flipped Classroom wurde eingesetzt, um im Fall eines möglichen Leistungseinbruchs in der Treatmentgruppe potentiell Aufklärungsarbeit leisten zu können.

Im Fragebogen wurde zusätzlich erhoben, ob sich in der Selbsteinschätzung der Schüler die häusliche Vorbereitungszeit im Flipped Classroom verändert hat. Über 65\% der Schüler gaben an, dass sich der Zeitaufwand für die häusliche Vorbereitung vergrößert hat (vgl. Tab. 31, Nr. 1). Der Widerspruch zum Befund in Kap. 6.4.2, nach dem sich die durchschnittliche Hausaufgabendauer nicht signifikant verändert hat, ist nur scheinbar.

Tab. 33. Mittlere Häufigkeit der Nutzung der Lernvideos mit integriertem Säulendiagramm

\begin{tabular}{|l|l|l|l|l|} 
& Nein, gar nicht & Nur zum Teil & Ja, vollständig & Ja, mehrmals \\
\hline ICh habe mir das Video angeschaut. & $\mathrm{M}=10.6 \%$ & $\mathrm{M}=14.1 \%$ & $\mathrm{M}=61.7 \%$ & $\mathrm{M}=13.6 \%$ \\
\hline & $\mathrm{SD}=4.4 \%$ & $\mathrm{SD}=6.7 \%$ & $\mathrm{SD}=9.5 \%$ & $\mathrm{SD}=9.3 \%$ \\
\hline
\end{tabular}




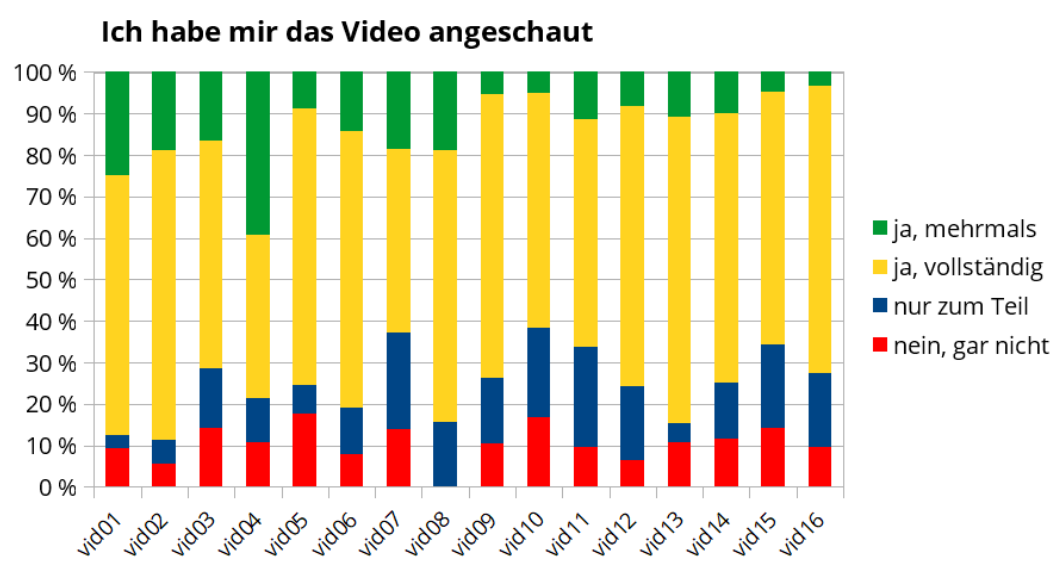

Abb. 3o. Nutzungsstatistik für die Lernvideos (Video 1-16)

Bei wesentlich besserer Hausaufgabendisziplin und gleichbleibender mittlerer Hausaufgabendauer vergrößert sich die insgesamt investierte häusliche Arbeitszeit im Flipped Classroom für die Schüler. Das Item ist jedoch unklar, weil für die Schüler nicht eindeutig ist, ob sich die Frage auf die durchschnittliche oder die gesamte häusliche Vorbereitungsdauer bezieht. Auf eine weitere Auswertung des Items muss deshalb verzichtet werden.

Des Weiteren wurden die Schüler um Einschätzung gebeten, ob sich durch die Unterrichtsmethode Flipped Classroom die Zeit verändert hat, die sie insgesamt vor einem Bildschirm verbracht haben. Das Ergebnis in Tab. 32 zeigt, dass Flipped Classroom nur eine geringe Auswirkung auf die Screen Time besitzt. Bei täglich durchschnittlich $1 \mathrm{~h} 41 \mathrm{~min}$ $(\mathrm{SD}=1 \mathrm{~h}$ 1omin $)$ Fernseh- und Streamingzeit und $2 \mathrm{~h} 9 \mathrm{~min}(\mathrm{SD}=1 \mathrm{~h} 17 \mathrm{~min})^{17}$ Zeit für andere internetbasierten Tätigkeiten, welche im Pretest für die Treatmentgruppe erhoben wurden, fällt die durchschnittliche Zeit von etwa 12 Minuten für die Hausaufgaben im Flipped Classroom (vgl. Tab. 28) tatsächlich nicht stark ins Gewicht. Allerdings weisen diese Werte eine starke Streuung auf, so dass für Schüler mit niedrigem Medienkonsum der relative Zuwachs durch die Lernvideos bedeutsam wird.

Zu Beginn jedes Clicker-Quiz wurde zusätzlich noch eine Frage gestellt, die den Umfang des Verständnisses der Lernvideos erhob. Gemittelt über alle 16 Videos ergibt sich die in Tab. 34 dargestellte Verteilung.

Tab. 34. Verständnis der Lernvideos (Schüleranteile) mit integriertem Säulendiagramm

\begin{tabular}{|c|c|c|c|c} 
& Nein, gar nicht & Nur zum Teil & Recht viel & Alles \\
\hline ICh habe die Inhalte des Videos verstanden. & $\mathrm{M}=6.5 \%$ & $\mathrm{M}=24.3 \%$ & $\mathrm{M}=42.4 \%$ & $\mathrm{M}=26.8 \%$ \\
& $\mathrm{SE}=4.6 \%$ & $\mathrm{SE}=14.6 \%$ & $\mathrm{SE}=8.9 \%$ & $\mathrm{SE}=15.5 \%$ \\
\hline
\end{tabular}

17 Die ordinalen Angaben der Schüler wurden zur besseren Veranschaulichung mit folgender Näherung umgerechnet: weniger als $1 / 2$ Stunde: $15 \mathrm{~min}, 1 / 2-1$ Stunde: $45 \mathrm{~min}, 1-2$ Stunden: $90 \mathrm{~min}, 2-3$ Stunden: $150 \mathrm{~min}$, 3-4 Stunden: $210 \mathrm{~min}$, mehr als 4 Stunden: $270 \mathrm{~min}$. 
Über 30\% der Schüler haben in dieser Skalierung die Videoinhalte nur zum Teil oder weniger verstanden. Der Großteil der Schüler konnte aber die Grundlagen der Videos zu Hause verarbeiten und verstehen. Auch hier gibt es keine Vergleichswerte zum traditionellen Unterricht. Es wäre auch schwierig, eine Parallele zu operationalisieren, da nicht eindeutig ist, worauf sich eine entsprechende Frage im traditionellen Unterricht beziehen sollte. Nachfolgende Statistik in Abb. 31 differenziert den Grad des Verständnisses nach Videos.

Besonders Video 7 (»Erzeugung von Wechselspannung «), Video 8 (»Effektivwerte der Wechselspannung «, Video 10 (»Ein- und Ausschaltvorgang bei einer Spule«) und Video 11 (»Magnetische Energie einer Spule «) $)^{18}$ bereiteten die größten Verständnisprobleme. Die Inhalte dieser Videos sind mathematisch anspruchsvoller, was die niedrigere Verstehensrate erklären könnte. Die Videos 1-6 besitzen vielfache Anknüpfungspunkte an die Lerninhalte der 9. Jahrgangsstufe, so dass den Schülern der Anschluss an vorheriges Wissen hier vermutlich am leichtesten fiel.

Die Inhaltsvermittlung über die Lernvideos war für einen gewissen Teil der Schüler eine Herausforderung. Sich eigenständig neue Inhalte über das Medium des Videos anzueignen, sagte nicht jedem zu. Eine echte Überforderung war diese Instruktionsform jedoch nicht, wie die Angabe zum Anspruch der Videos (vgl. Tab. 31, Nr. 2) zeigt. Im freien, stichpunktartigen Feedback benannten die Schüler im Flipped Classroom drei große Nachteile: Die Videos erschienen vielen teilweise unverständlich oder nicht gut genug erklärt $(\mathrm{N}=20 \mid 28,57 \%)$, es gab im Vergleich zum traditionellen Unterricht keine Möglichkeit zur Nachfrage oder Interaktion $(\mathrm{N}=18 \mid 25.71 \%)$ und der häusliche Aufwand sei höher gewesen als im traditionellen Unterricht $(\mathrm{N}=17 \mid 24.29 \%)$. Auf der positiven Seite jedoch schätzten die Schüler an den Lernvideos, dass sie sie im eigenen Tempo und mit Wiederholungen ansehen konnten $(\mathrm{N}=25 \mid 35.71 \%)$, mit ihnen versäumte Inhalte nachholen und sie zur Klausurvorbereitung nutzen konnten $(\mathrm{N}=19$ | 27.14\%), da sie mit ihnen eine gute, strukturierte und kompakte Aufbereitung der Unterrichtsinhalte besaßen $(\mathrm{N}=15 \mid 21.4 \%)$.

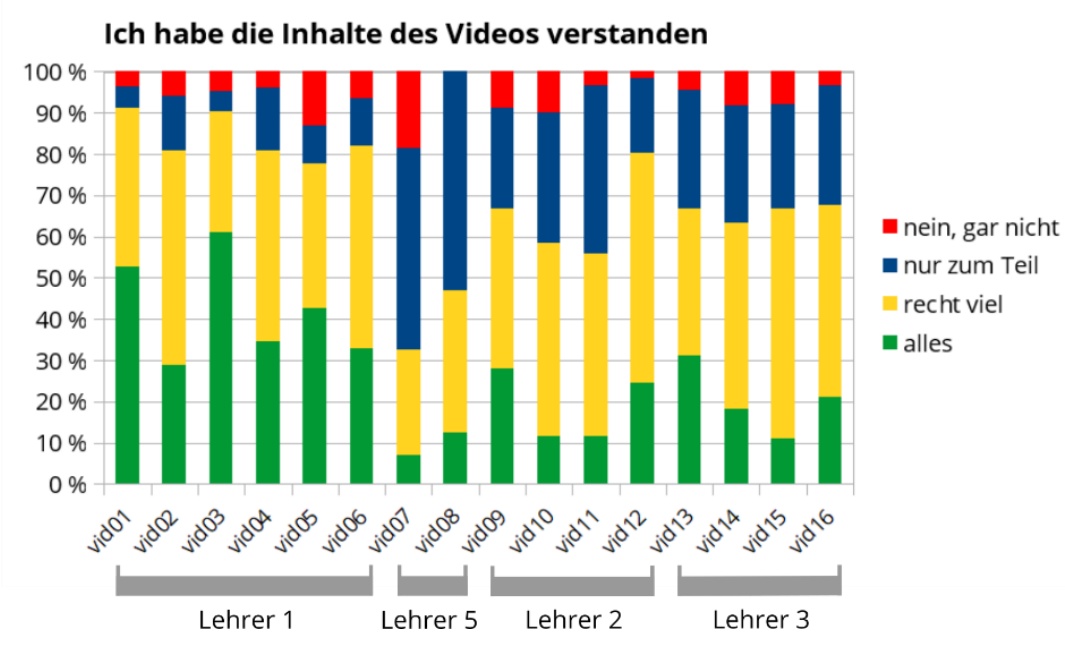

Abb. 31. Verständnisstatistik für die Lernvideos (Video 1-16) unter Angabe des Produzenten

18 Vgl. Kap. 4.3. 


\subsection{2 Übungsaufgaben im Unterricht und Schüleraktivierung}

Ein weiteres charakteristisches Element im Flipped Classroom ist die ausgedehnte Übungszeit im Unterricht. Die Übungszeit wurde in dieser Unterrichtsreihe genutzt, um Probleme, Aufgaben und Anwendungsbeispiele zu bearbeiten, also in erster Linie fachliche Kompetenzen zu fördern.

In den Kursen der Kontrollgruppe dokumentierten alle Lehrer, welche Aufgaben sie für die Schüler im Unterricht und in der Hausaufgabe zur Bearbeitung vorsahen, in den Treatmentkursen liegt die Dokumentation nur von den Lehrern 1-3 vor, deren Aufgabeneinsatz jedoch identisch war. Zwar gab es - gemäß dem Studiendesign - Freiräume für die Lehrer bei der Wahl der Aufgaben, doch hat es sich durch die starke Synchronisierung des Unterrichts in Schule A ergeben, dass die Lehrer 1-3 stets die gleichen Aufgaben in den Kontroll- und Treatmentkursen eingesetzt haben. Viele Aufgaben beinhalteten Teilaufgaben, so dass die Summe der Teilaufgaben ein quantitatives Maß für den Vergleich der Unterrichtsformate darstellt.

Aufgrund von Ausfallstunden unterscheidet sich die Anzahl der Unterrichtsstunden von Kontroll- und Treatmentgruppe. In Tab. 35 wird der Vergleich der Teilaufgabenanzahl dargestellt. Die Angaben in der ersten Spalte beziehen sich nicht darauf, wie viele Teilaufgaben von allen Schülern bearbeitet wurden, da sowohl in der Kontrollgruppe als auch im Flipped Classroom Wahlpflichtaufgaben zur Verfügung gestellt wurden. Die Summe der Teilaufgaben bezieht sich vielmehr auf die Gesamtzahl der Aufgaben, welche den Schülern zur Verfügung gestellt wurden und von den Schülern mit dem größten Arbeitstempo bearbeitet werden konnten. Dennoch dienen sie als grobes Vergleichsmaß, bei dem deutlich wird, dass im Unterricht des Flipped Classroom mehr als doppelt so viele Teilaufgaben eingesetzt werden konnten als im Unterricht und den Hausaufgaben des klassischen Formats. Im Flipped Classroom hatten die Schüler durch die Vielzahl an Aufgaben deutlich mehr Gelegenheit, zum richtigen Verständnis zu gelangen und die Lerninhalte einzuüben und anzuwenden.

Den Vorteil des intensiven Übens und Anwendens hoben die Schüler auch im freien Feedback besonders hervor. Kein anderer Nutzen wurde häufiger genannt als die Möglichkeit im Flipped Classroom, mehr zu üben und zu rechnen $(\mathrm{N}=25 \mid 35.71 \%)$.

Tab. 35. Vergleich der Anzahl der eingesetzten Teilaufgaben in der Unterrichtsreihe

\begin{tabular}{|c|c|c|c|c|}
\hline & $\begin{array}{l}\text { Mittlere Summe der ein- } \\
\text { gesetzten Teilaufgaben }\end{array}$ & SD & $\begin{array}{c}\text { Anzahl der } \\
\text { Unterrichtsstunden }\end{array}$ & $\begin{array}{l}\text { Teilaufgaben pro } \\
\text { Unterrichtsstunde }\end{array}$ \\
\hline traditionell & 53.8 & 11.0 & 23 & 2.3 \\
\hline flipped & 123 & $-{ }^{19}$ & 25 & 4.9 \\
\hline
\end{tabular}

19 Für die Treatmentkurse des Flipped Classroom lag nur von den Lehrern 1-3 eine Unterrichtsdokumentation vor. Diese Lehrer verwendeten jedoch identische Arbeitsblätter, so dass es zwischen den Kursen keine Abweichungen gab. 
Die hohe Schüleraktivierung spiegelt sich auch in den Fragebogenergebnissen wieder. Über die Hälfte der Schüler fühlte sich stärker aktiviert und in die Pflicht genommen als im traditionellen Unterricht und etwa 30\% geben an, im Flipped Classroom nicht aktiver mit ihren Mitschülern gearbeitet zu haben als im normalen Unterricht. (vgl. Tab. 31, Nr. 3). Auch das stärkere kooperative Element des Flipped Classroom findet seinen Niederschlag in den Äußerungen der Schüler (vgl. Tab. 31, Nr. 4). Ungeachtet dessen schätzten jedoch fast $45 \%$ der Schüler ihr Unterrichtsengagement nicht höher ein als sonst, Im freien Feedback wurde das Clicker-Quiz als aktivierende Einstiegsmethode, mit der die Inhalte der Lernvideos wiederholt und überprüft wurden, positiv geschätzt. 16 Schüler (22.9\%) sahen im Clicker-Quiz einen hilfreichen Wissenstest. Gemessen an allen positiven Rückmeldungen war das - nach den oben bereits erwähnten - die vierthäufigste Nennung. 13 Schüler (18.6\%) empfanden die ClickerQuizze jedoch teilweise als zu lang, obschon sie nach Methodenkonzept generell auf zehn Minuten begrenzt waren.

\subsubsection{Experimente im Physikunterricht}

Gemäß Studiendesign sahen die Schüler im Flipped Classroom die gleichen Experimente wie die Schüler der Kontrollgruppe im traditionellen Unterricht, wenn auch nur im Lernvideo statt live. Die vorliegende Studie unterscheidet sich von allen anderen Studien zum Flipped Classroom im naturwissenschaftlichen Bereich dadurch, dass reale Experimente Teil der Lernvideos waren. Viele Versuchsaufbauten waren dabei für die Kontroll- und Treatmentgruppe identisch, manche wurden für die Videografie modifiziert. Durch das Medium des Videos hatten die Schüler uneingeschränkte Sicht auf die videografierten Experimente, oft gab es Großaufnahmen von wichtigen Details. Aufgrund der engen Zeitvorgaben blieb jedoch für die Beschäftigung mit dem Experiment weniger Zeit als im traditionellen Unterricht. Das Lernen am Experiment im Sinne des forschend-entdeckenden Unterrichts (Höttecke \& Rieß 2015) nahm in den Lernvideos naturgemäß einen quantitativ wesentlich geringeren Umfang ein.

Nach Tab. 31, Nr. 5 stimmten etwa 50\% der Schüler der Aussage zu, dass sie einen größeren Nutzen von Live-Experimenten gehabt hätten, die andere Hälfte stimmte dieser Aussage nicht zu. Im freien Feedback gaben 15 Schüler $(21.4 \%)$ explizit an, dass sie Live-Experimente im Unterricht vermisst hätten. Es zeigt sich damit keine eindeutige Tendenz. In den Videos wurden Experimente stets als Basis physikalischen Lehrens verwendet. Sie bildeten den Ausgangspunkt und das Kriterium physikalischer Erkenntnisgewinnung. Die Art, wie reale Experimente in die Videos integriert wurden, führte immerhin bei der Hälfte der Schüler zu der Einschätzung, keinen größeren Nutzen durch Live-Experimente im Unterricht zu haben. Bei reinen Voice-over-Screencasts wäre diese Quote mutmaßlich geringer. Über die Motivlage, warum Schüler lieber Live- statt videografierte Experimente gesehen hätten oder warum sie demgegenüber indifferent waren, lässt sich in dieser Studie jedoch keine Aussage treffen. 


\subsubsection{Veränderte Lehrerrolle}

Der überraschende Befund zur wahrgenommenen Lehrerunterstützung, dem gemäß Нypothese H3a abzulehnen ist (vgl. Kap. 6.4.1), bestätigt sich in den Rückmeldungen der Schüler, die auf das Lehrerverhalten bezogen sind. Demnach glaubte nur weniger als die Hälfte der Schüler, dass ihr Physiklehrer im Flipped Classroom ein besseres Bild davon habe, was sie können und was nicht (vgl. Tab. 31|6). Etwa ein Drittel stimmte dieser Aussage eher nicht zu, fast ein Viertel überhaupt nicht. Dafür ist der Anteil der Schüler, nach denen der Physiklehrer ihnen bei Problemen besser individuell helfen konnte, mit 55.7\% höher als der Anteil der Schüler, die anderer Meinung waren (vgl. Tab. 31, Nr. 7). Im freien Feedback hoben 15 Schüler $(21.4 \%)$ hervor, dass sie die schnelle Hilfestellung durch den Lehrer oder die Mitschüler im Flipped Classroom schätzten.

Die für den traditionellen Unterricht typische Form des Lehrer-Schüler-Gesprächs, das zwischen dem Lehrer und dem ganzen Kurs stattfindet, wurde von den meisten Schülern im Flipped Classroom vermisst (vgl. Tab. 31, Nr. 8). 64.3\% der Schüler sahen hier einen Nachteil der Unterrichtsmethode. Im freien Feedback wurde als großer Nachteil genannt, dass es im Unterricht zu wenig Erklärungen und Diskussionen im Plenum und kein gemeinsames Erarbeiten und Herleiten von Formeln gab $(\mathrm{N}=17 \mid 24.3 \%)$. Diese Kritik bezieht sich jedoch weniger auf die Art der Lehrer-Schüler-Interaktion. Sie ist vielmehr gegen das Fundament des Flipped Classroom gerichtet und repräsentiert die Schwierigkeit mancher Schüler, sich im Flipped Classroom auf Änderungen in den Unterrichts- und Lerngewohnheiten einzulassen. Auch in Studien anderer Länder zum Flipped Classroom wurde beobachtet, dass ein Teil der Schüler und Studenten deutlich den Wunsch artikulierte, klassisch und lehrerzentriert unterrichtet zu werden (Hao 2016; Şengel 2016).

\subsubsection{Weitere Rückmeldungen}

Die von einigen Schülern formulierte Kritik an Elementen des Flipped Classroom soll abschließend in den Kontext einer Gesamteinschätzung der Unterrichtsmethode durch die Schüler gebracht werden. Wie bei mehreren Feedback-Items im Fragebogen teilen sich auch im offenen Feedback die Schüler in der Frage, ob sie im Flipped Classroom im Vergleich zum normalen Unterricht mehr gelernt hätten, in zwei Lager (vgl. Tab. 31, Nr. 10). Etwas weniger als die Hälfte der Schüler (45.7\%) schätzt ihren Lernerfolg höher ein als im traditionellen Unterricht, der Rest der Schüler (54.3\%) teilt diese Einschätzung nicht. Die Verteilung ist dabei sehr symmetrisch: $11(15.7 \%)$ beziehungsweise 13 Schüler (18.6\%) hatten eine sehr klare positive beziehungsweise negative Haltung zu der Frage, jeweils etwa doppelt so viele Schüler besaßen lediglich eine Tendenz zur positiven beziehungsweise negativen Haltung.

Mit einer negativen Haltung ist nicht zwangsläufig eine Ablehnung der Methode verbunden wie etwa bei Butt (2014), in dessen Studie sich eine Methodenablehnungsquote von 25\% zeigte. Aber im freien Feedback gab es einzelne offene Ablehnungen dieser Methode, die damit begründet wurden, dass weniger verstanden wurde $(\mathrm{N}=4 \mid 5 \cdot 7 \%)$ oder die Methode als $z u$ 
ineffizient eingeschätzt wurde $(\mathrm{N}=3 \mid 4.3 \%)$. Dem entgegen steht die Einschätzung anderer Schüler, die die Methode als effizienter erachten als den traditionellen Unterricht $(\mathrm{N}=6 \mid 8.6 \%)$ und die höhere Eigenverantwortung und Selbstständigkeit hervorhoben $(\mathrm{N}=4 \mid 5 \cdot 7 \%)$.

Ein großer Teil der Schüler empfand die Methode für den Zeitraum von acht Wochen - mit Schulferienunterbrechungen waren es drei Monate - als zu monoton (42.8\%), die übrigen Schüler empfanden die Methode nicht als zu eintönig (57.2\%, vgl. Tab. 31, Nr. 9).

\subsubsection{Diskussion}

Aus den anonymen Angaben zu den Clicker-Fragen, die zu Beginn jedes Quiz im Unterricht gestellt wurden, und den pseudonymen Angaben zu den geschlossenen Fragebogen-Items des Posttests lässt sich schließen, dass ein Großteil der Schüler immer alle Videos zur Vorbereitung geschaut und das meiste davon auch verstanden hat. Damit können - sozusagen nachträglich - wichtige Voraussetzungen validiert werden, unter denen sich im Flipped Classroom Lernprozesse initiieren lassen. Hätten viele Schüler die Lernvideos nicht angesehen oder zum überwiegenden Teil nicht verstanden, wäre zu überlegen gewesen, welche fachdidaktischen oder pädagogischen Entscheidungen falsch getroffen wurden.

Die weiteren Aspekte, zu denen die Schüler befragt wurden, zeichnen kein eindeutiges Bild. Die Schüler waren ambivalent in der Frage, ob sich der häusliche Aufwand vergrößert hat, ob ihre Aktivität im Unterricht stärker geworden ist, ob sie im Flipped Classroom mehr gelernt haben als im traditionellen Format, ob sie von Live-Experimenten mehr profitiert hätten, ob der Lehrer ein genaueres Bild von ihren Fähigkeiten bekommen hat, ob der Lehrer bei Problemen besser auf sie eingehen konnte oder ob die Methode für diesen Zeitraum zu monoton war. In keinem dieser Aspekte manifestiert sich eine eindeutige Richtung in den Schüleräußerungen. Die Diversität in der Rezeption des Flipped Classroom entspricht der Vielfalt persönlicher Motive der Schüler und der Vielfalt ihrer eigenen Vorerfahrungen.

Im freien, stichpunktartigen Feedback zum Posttestzeitpunkt formulierten die Schüler als größte Nachteile des Flipped Classroom, dass

(a) die Instruktionsqualität der Videos oft zu wünschen übriggelassen habe,

(b) es durch die Videos keine Möglichkeit zur Nachfrage gegeben habe und

(c) der häusliche Aufwand höher gewesen sei.

Die ersten beiden Kritikpunkte hängen dabei möglicherweise zusammen. Die Erstellung der Lernvideos oblag den einzelnen Lehrkräften, die durch ihren je individuellen Erklär- und Präsentationsstil die Videos prägten. Erschließt sich nun durch eine bestimmte Art der Erklärung im Video ein physikalischer Zusammenhang nicht für einen Schüler, hat er keine Möglichkeit, direkt nachzufragen, und lastet sein Unverständnis der Qualität der Erklärung im Video an. Hier liegt tatsächlich eine objektive Kritik am Flipped Classroom. Durch das asynchrone Lernen am Video müssen Fragen schriftlich notiert und auf die Präsenzzeit verschoben werden. Oft aber bauen Lernstufen im Video aufeinander auf. Fehlt eine Stufe, können auch 
die folgenden nicht genommen werden. Um diesem Problem zu begegnen, sind hier Erweiterungen des Flipped Classroom denkbar, in denen die Funktionen eines geschlossenen Onlineforums in Lernmanagementsystemen wie MEBIS oder Moodle genutzt werden können, in dem sich die Schüler vorab miteinander oder mit dem Lehrer austauschen können.

In der Präsenzphase des Flipped Classroom hat das klassische Lehrer-Schüler-Gespräch keinen Platz. Diese Tatsache sehen fast zwei Drittel der Schüler als Nachteil der Methode. Die Frage, warum die Mehrzahl der Schüler des Flipped Classroom die Lehrer-Schüler-Gespräche im Plenum vermisst, lässt sich in dieser Studie nicht beantworten. Gründe hierfür liegen sicher nicht in der schlechteren Lernunterstützung im Flipped Classroom. Möglicherweise erhoffen sich die Schüler in den klassischen Lehrer-Schüler-Gesprächen eine vermeintlich höhere »Gerechtigkeit« an Lehreransprache und -zuwendung. In den Augen der Schüler erhalten in Plenumsgesprächen alle dieselbe Unterstützung vom Lehrer, dieselben Hinweise, dieselben Hilfen, um Wesentliches vom Unwesentlichen zu trennen, profitieren alle in gleicher Weise von den Fragen einzelner Schüler. Die Forderung, dass niemand bevorzugt und benachteiligt wird, ist gerade vor dem Hintergrund der Klausur- und Abiturrelevanz der Unterrichtsinhalte nachzuvollziehen. Jedem Schüler gerecht zu werden bedeutet jedoch gerade nicht, allen das Gleiche, sondern jedem das Seine zukommen zu lassen.

Ein weiterer Aspekt hinter dem Bedürfnis nach Lehrer-Schüler-Gesprächen könnte sein, dass jedes Plenumsgespräch den Schülern erlaubt, ihr je eigenes Verhältnis von Unterrichtsaktivität und -passivität selbst zu bestimmen. Während der Flipped Classroom wenig Freiräume lässt, sich passiv zu verhalten, lädt im Vergleich dazu der traditionelle Unterricht mit seinen Lehrervorträgen und Plenumsgesprächen eher dazu ein, sich zurückzunehmen und weniger aktiv zu sein. Für Schüler bietet das vordergründig eine attraktive Möglichkeit zur Entlastung im Unterricht.

Die letztgenannte Kritik der Schüler am größeren häuslichen Aufwand im Flipped Classroom lässt sich konsistenterweise nur auf die Gesamtheit häuslicher Tätigkeit beziehen. Wie in Kap. 6.4.2 gesehen, ließ sich zeigen, dass die mittlere Hausaufgabendauer pro Unterrichts(doppel)stunde konstant geblieben ist und sich lediglich die Hausaufgabendiszplin erhöht hat. Wenn Schüler als Kritik anbringen, dass sich durch Flipped Classroom der häusliche Aufwand erhöht habe, bezieht sich das auf die eigene erhöhte Hausaufgabendisziplin. Statistisch wird sie durch andere Schüler ausgeglichen, die angaben, dass sich der häusliche Aufwand für sie verringert habe.

Als größte Vorteile des Flipped Classroom nannten die Schüler im freien, stichpunktartigen Feedback, dass

(a) durch die Videos eine Instruktion im eigenen, selbstbestimmten Tempo möglich gewesen sei,

(b) die Inhalte leicht nachzuholen und zu rekapitulieren gewesen seien und

(c) im Unterricht wesentlich mehr geübt worden sei.

Die Unterrichtsdokumentation der beteiligten Lehrer bestätigt den letzten Punkt quantitativ. Durch die Vorverlagerung der Instruktion in die häusliche Tätigkeit wurden zeitliche 
Freiräume geschaffen, die es den Schülern im Flipped Classroom ermöglichten, mehr als doppelt so viele Übungsaufgaben zu bearbeiten als im Vergleichsunterricht der Kontrollgruppe.

Die Schülereinschätzungen der positiven Seiten des Flipped Classroom lassen sich direkt in Beziehung setzen zu den Grundintentionen dieser Unterrichtsmethode. Der Kerngedanke des Flipped Classroom - die Schüleraktivierung - findet seinen Ausdruck in der intensiven Partner- und Gruppenarbeit, in der die Schüler eine Vielzahl an physikalischen Problemen und Aufgaben gelöst haben. Zudem wird die Möglichkeit, Lernvideos anzuhalten, in ihnen zurückzuspringen und wieder vorzuspringen, von den Schülern wertgeschätzt. Die Kontrolle über die kognitive Belastung durch die Lernvideos (vgl. Cognitive Load Theory, Kap. 2.3.3) und das selbstgesteuerte und -bestimmende Lernen, die den Schülern durch das Onlineformat der Videos und die Wahlaufgaben im Unterricht in gewissem Maße ermöglicht wird (vgl. Selbstbestimmungstheorie, Kap. 2.3.3), ist eine wesentliche Stärke des umgedrehten Unterrichtsformats im Flipped Classroom. Die Schüler haben mit der Selbstbestimmung durch das Videolernen dezidiert einen intendierten Aspekt des Flipped Classroom hervorgehoben.

Abschließend soll der Befund diskutiert werden, dass fast die Hälfte der Schüler den Flipped Classroom für acht Unterrichtswochen als zu monoton empfand. Positiv gesehen stützt diese Einschätzung die im Studiendesign verfolgte Absicht, dass zum Zeitpunkt des Posttests kein motivationaler Neuheitseffekt (vgl. Clark 1983) mehr eine Rolle spielen soll, der die kognitiven und affektiven Lernergebnisse beeinflusst. Negativ gesehen weist der Befund darauf hin, dass der Flipped Classroom im Physikunterricht keine Dauermethode sein sollte, wenn sich die Unterrichtsaktivität auf das Bearbeiten von Aufgaben und Lösen von Problemen beschränkt. Im Rahmen der Lernzielsetzung und des Studiendesigns wurde diese Aktivitätsbeschränkung explizit in Kauf genommen, weil ausdrücklich die Förderung und Erhebung des deklarativen Wissens im Vordergrund stand. Doch für den generellen Einsatz im Physikunterricht der Schule, der noch weitere Kompetenzebenen im Blick hat, sind eine zeitliche Beschränkung oder der Einsatz anderer schüleraktivierender Tätigkeiten in der Präsenzphase dringend erforderlich. Möglicherweise trug auch der Eindruck, viele Wochen in einer methodischen Großform unterrichtet worden zu sein, dazu bei, dass sich das Interesse der Schüler am Unterrichtsfach Physik im Flipped Classroom ebenso negativ entwickelte wie im klassischen Unterricht der Kontrollgruppe, wie Kap. 6.2.2 gezeigt hat. Ein kontinuierliches Monitoring der aktuellen Motivation oder des situationalen Interesses könnte hier in einer Folgestudie Aufklärung leisten. 


\section{Zusammenfassung und Ausblick}

Die junge Unterrichtsmethode Flipped Classroom wird in der deutschsprachigen Bildungslandschaft eher im Hochschulbereich eingesetzt, findet aber zunehmend Verbreitung im Schulbereich. Die Auswirkung der Methode auf kognitive und affektive Lernerfolge im deutschsprachigen Schulunterricht im Allgemeinen oder im Physikunterricht im Besonderen waren bislang nicht Gegenstand fach- oder physikdidaktischer Untersuchungen. Hier versuchte die vorliegende Arbeit, einen Beitrag zu leisten und untersuchte die Wirkungen des Flipped Classroom im Physikunterricht der Oberstufe.

Wichtig ist zu betonen, dass es nicht um den Vergleich der Instruktionsformen »Lernvideo « versus »Lehrervortrag « und damit nicht um den - eher problematischen - Vergleich von $\mathrm{Me}$ dien geht (vgl. Clark 1994), sondern um den Vergleich von Methoden, der eng genug an Lernziele, Lernvoraussetzungen und Lerninhalte gebunden ist, um didaktisch legitim zu sein. Zentrale Voraussetzung der Studie ist, dass der Flipped Classroom nur als Einheit von häuslicher Videoinstruktion und schulischer Aktivierung der Schülerinnen und Schüler gesehen werden kann. Dabei standen sowohl kognitive (Fachwissen) als auch affektive Lernerfolge (Motivation, Interesse, Selbstkonzept) im Fokus der Studie sowie die Frage nach Veränderungen sogenannter Stützfaktoren des Unterrichts (Lehrerunterstützung, Hausaufgabendiszplin und dauer). Zusätzlich wurde untersucht, ob die Wirkung des Flipped Classroom durch weitere Faktoren moderiert wird (Leistungsniveau, Geschlecht, Lehrer).

In der quasiexperimentellen Studie mit N = 151 Schülern und fünf Lehrkräften in acht Physikkursen an zwei Gymnasien wurden die Wirkungen des Flipped Classroom mit denen des traditionellen Unterrichts verglichen. Die Interventionsstudie umfasste acht Schulwochen und wurde in zwei aufeinander folgenden Schuljahren in der 11. Jahrgangsstufe durchgeführt.

Sie zeichnet sich gegenüber den wenigen physikbezogenen Flipped Classroom-Studien anderer Länder dadurch aus, dass sie im Physikunterricht einer allgemeinbildenden Schule (amerikanisch: High-School, englisch: Grammar School) stattfand, mehr als nur ein einzelner Lehrer beteiligt war, affektive Lernergebnisse kontrolliert gemessen wurden und Realexperimente in die Lernvideos integriert waren. Während in Lernvideos zum Physikunterricht sonst reine Voice-over-Screencasts ohne Experimente eingesetzt werden, erstellten die an der Studie beteiligten Lehrer Videos, die Voice-over-Screencasts mit videografierten Realexperimenten organisch verbanden, um eine höhere Physikauthentizität und eine bessere Vergleichbarkeit zu Instruktionsphasen des schulischen Physikunterrichts zu erzielen. 


\subsection{Ergebnisse in zehn Punkten}

Die wichtigsten Ergebnisse der Studie lassen sich in zehn Punkten zusammenfassen:

1. Die Lernleistung im Flipped Classroom war signifikant höher als im traditionellen Unterricht. Die Effektstärke lag im mittleren Bereich.

2. Das Selbstkonzept der Schüler im Flipped Classroom wurde signifikant besser. Im traditionellen Unterricht blieb es unverändert. Der Effekt ist jedoch klein.

3. Die Motivation, sich mit Physik zu beschäftigen, fiel im Flipped Classroom im Gegensatz zur Kontrollgruppe nicht ab. Der Effekt ist klein.

4. Das Interesse am Unterrichtsfach Physik ging in beiden Gruppen zurück. Hier gab es keine Unterschiede zwischen Flipped Classroom und traditionellem Unterricht.

5. Die wahrgenommene Lehrerunterstützung änderte sich im Flipped Classroom nicht im Vergleich zum traditionellen Unterricht.

6. Die Hausaufgabendisziplin wurde im Flipped Classroom signifikant besser. Die Effektstärke lag im mittleren Bereich. Die mittlere Hausaufgabendauer änderte sich jedoch nicht.

7. Das Leistungsniveau der Schüler beeinflusste nicht die Wirkung des Flipped Classroom auf die Lernleistung, die Motivation, das Fachinteresse und das Selbstkonzept.

8. Das Geschlecht der Schüler hatte keinen Einfluss auf die Wirkung des Flipped Classroom. Lediglich in der Motivation und in der Hausaufgabendisziplin gab es geschlechtsabhängige Unterschiede: Im Flipped Classroom hatten die Mädchen eine signifikant höhere Motivation als die Mädchen im traditionellen Unterricht (große Effektstärke), während die Jungen im Flipped Classroom eine deutlich bessere Hausaufgabendisziplin besaßen als im traditionellen Unterricht (mittlere Effektstärke).

9. Aussagen über den Einfluss der Lehrer lassen sich mit der Stichprobengröße nicht belastbar treffen. Wenn man den Faktor Lehrer dennoch berücksichtigt, stellt man fest, dass die einzelnen Lehrkräfte keinen Unterschied machten in der Wirkung des Flipped Classroom. Lediglich für die Änderung der Hausaufgabendisziplin und -dauer spielte es eine Rolle, welcher Lehrer im Flipped Classroom unterrichtet hatte.

10. Die Angaben und Rückmeldungen der Schüler zeigten, dass etwa $75 \%$ der Schüler die Videos vollständig angesehen und größtenteils verstanden hatten. Der Gesamteindruck der Schüler vom Flipped Classroom war nicht einheitlich. Als positive Elemente der Methode wurden das intensive und kooperative Üben und Anwenden der Lerninhalte, das selbstbestimmte Lerntempo und die kompakte Aufbereitung der Inhalte in den Lernvideos genannt. Als negativ wurden teilweise die Instruktionsqualität der Videos, die fehlende Möglichkeit zu direkten Nachfragen zum Video und Plenumsgesprächen im Unterricht sowie der erhöhte häusliche Aufwand empfunden. Fast die Hälfte der Schüler vermisste echte Realexperimente im Unterricht und empfand die Unterrichtsmethode für den Zeitraum von acht Schulwochen als zu monoton. Der Eindruck des intensiven Übens wird durch die Unterrichtsdokumentation der Lehrer 
quantitativ bestätigt. Demnach wurden im Flipped Classroom mehr als doppelt so viele Aufgaben pro Unterrichtsstunde bearbeitet wie im traditionellen Unterricht.

Einige dieser Befunde passen - wie in den entsprechenden Diskussionsteilen gezeigt - zu den positiven Ergebnissen internationaler Flipped Classroom-Studien, die in der universitären Ausbildung im Fach Physik oder in der schulischen Ausbildung in anderen Fachdisziplinen gefunden wurden. Die Studie konnte demnach zeigen, dass Flipped Classroom auch im Physikunterricht der deutschen Oberstufe einen Mehrwert für den kognitiv-fachlichen Wissenszuwachs bietet. Ein »Mehr« an physikalischem Fachwissen lässt sich durch Flipped Classroom nicht nur in universitären Lehrformen erzielen, sondern auch im allgemeinbildenden Schulunterricht. Flipped Classroom hat dabei auch einen positiven Einfluss auf das physikbezogene Selbstkonzept und die Motivation der Schüler, sich mit Physik zu beschäftigen, womit ein neuer Mosaikstein das Bild ergänzt, das sich fachdidaktisch vom Flipped Classroom konstruieren lässt. Der lernpsychologische Zusammenhang zwischen kognitiven und affektiven Lernprozessen (vgl. Kap. 2.2) tritt auch in dieser Unterrichtsmethode zum Vorschein, wenn auch nicht in dem Umfang und der Deutlichkeit, wie in den Hypothesen erwartet wurde.

Des Weiteren zeigte die Studie, dass Flipped Classroom im Physikunterricht eine breitbandig fördernde Unterrichtsmethode ist, von der leistungsschwache Schüler genauso profitieren wie leistungsstarke. Zudem wurde deutlich, dass es keinen Geschlechtereffekt gab. Sowohl Jungen als auch Mädchen haben gegenüber dem jeweils anderen Geschlecht keinen Vor- oder Nachteil. Lediglich im Bereich der intrinsischen Motivation, sich mit Physik zu beschäftigen, hatte der Flipped Classroom bei Mädchen einen stärkeren Effekt als bei Jungen. Angesichts der Stichprobengröße darf dieser Befund jedoch nicht uneingeschränkt generalisiert werden. Folgestudien könnten Wirkzusammenhänge hier genauer untersuchen.

Ebenfalls bedeutsame Ergebnisse liegen in den Stützfaktoren vor. Die Schüler im Flipped Classroom fühlten sich durch ihre Lehrer nicht stärker unterstützt und besser begleitet als im traditionellen Unterricht. Dieser Befund war überraschend und steht im Gegensatz zu bisherigen Studien zum Flipped Classroom, die diese Ebene untersucht haben. Vermutlich war das Lehrerhandeln bereits im traditionellen Physikunterricht durch ein hohes Maß an Zugewandtheit und Unterstützung ausgezeichnet, denn schon der Messwert vor Beginn des Treatments lag auf einem hohen Niveau. Ob das Ergebnis zur Lehrerunterstützung nur für den Physikunterricht der an der Studie beteiligten Lehrer gilt oder für den durchschnittlichen Schulunterricht in Deutschland, lässt sich nicht entscheiden. Systematische Unterrichtsbeobachtung könnten hier Aufklärung leisten. Der zweite Aspekt, der Beachtung verdient, ist die deutlich gesteigerte Hausaufgabendisziplin, vor allem bei den Jungen. Der Anreiz der häuslichen Vorbereitung (Lernvideos als Vorbereitung schauen) war im Vergleich zu den traditionellen Hausaufgaben (Aufgaben zur Nachbereitung bearbeiten) offenbar deutlich niederschwelliger. Möglicherweise erschien den Schülern auch die Bedeutung der häuslichen 
Tätigkeit im Flipped Classroom bedeutsamer als im traditionellen Unterricht. Der zeitliche Einsatz der Schüler war aber in beiden Fällen der gleiche. ${ }^{20}$

Ausdrücklich keine Forschungsfrage der vorliegenden Studie war zu untersuchen, welche Wirkmechanismen im Flipped Classroom im Einzelnen eine Rolle im Physikunterricht spielen. Flipped Classroom ist eine methodische Großform, die eine Reihe von schüleraktivierenden und unterrichtsbeeinflussenden Maßnahmen bündelt. Die Verwendung von Lernvideos, der niederschwellige Anreiz zur häuslichen Vorbereitung, der Einsatz von Quizze in einem Peer-Education-Setting, die veränderte Lehrerrolle als Lernbegleiter, die Förderung von selbstverantwortlichem Lernen und die gesteigerte soziale Bezogenheit in den kollaborativen Arbeitsformen sind Merkmale des Flipped Classroom, deren Einzelwirkungen hier nicht untersucht werden sollten. Aufgrund der Komplexität von schulischen Lernprozessen stellt sich die Frage, inwieweit sich mögliche Einzelwirkungen überhaupt voneinander abgrenzen lassen. Flipped Classroom ist ein Gesamtpaket, das für den Zweck dieser Studie eine bestimmte Form annahm (vgl. Kap. 2.3.2) und als Ganzes in seiner Wirkung betrachtet wurde.

Die lernpsychologische Voraussetzung erfolgreichen Unterrichts liegt in der kognitiven Aktivierung. Wo kognitive Aktivierung in den Tiefenstrukturen des Unterrichts durch Flipped Classroom begünstigt wird, kann von gelingendem Lernen gesprochen werden (vgl. Kap. 2.1). Zwar lässt sich kognitive Aktivierung grundsätzlich operationalisieren und durch Unterrichtsbeobachtung indirekt messen, doch war die systematische Unterrichtsbeobachtung und die Messung der kognitiven Aktivierung kein Erhebungsverfahren, das in dieser Studie eingesetzt wurde. Stattdessen wurden einerseits die affektiven Lernvoraussetzungen der kognitiven Aktivierung sowie andererseits das Ergebnis des durch kognitive Aktivierung vermittelten Lernprozesses gemessen. Das Forschungsinteresse lag deshalb nicht auf der rein lernpsychologischen Frage, ob Flipped Classroom zu höherer kognitiver Aktivierung als traditioneller Unterricht führt, sondern auf der fachdidaktischen Frage, welche Auswirkungen Flipped Classroom im Physikunterricht der Oberstufe besitzt. Die Wirkungsvermittlung durch kognitive Aktivierung wurde dabei mit guten Gründen angenommen (vgl. Kap. 2.3.3). Die positiven Ergebnisse hinsichtlich der Lernleistung und des Selbstkonzepts, das eng mit der eigenen Leistung zusammenhängt, lassen sich demnach auf die erhöhte kognitive Aktivierung durch den Flipped Classroom zurückführen.

20 Offen ist die Frage, wie sich die Schüler in einem Physikunterricht eines Lehrers verhalten, der üblicherweise keine Hausaufgaben erteilt. Hier wird durch die Lernvideos kein Ersatz der häuslichen Tätigkeit gebracht, sondern eine zusätzliche Belastung. In diesem Fall muss der Einsatz von Flipped Classroom anders gedacht werden, zum Beispiel als In-class-Flip, bei dem die Schüler zu Beginn der Stunde ein Lernvideo im eigenen Tempo und auf eigenen Geräten ansehen. 


\subsection{Kritische Betrachtung der Untersuchung}

Im Untersuchungsdesign der Studie lassen sich Konzeptmerkmale finden, die nicht unproblematisch sind. Sie berühren die Güte der Untersuchung, so dass es für das Gesamtbild der Befunde wichtig ist, sie zu benennen.

\section{Externe Validität}

Die Stichprobe ist mit N = 151 Schülern und fünf Lehrkräften nicht sehr groß. Für eine höhere externe Validität der Studie und bessere Generalisierbarkeit der Ergebnisse wäre ein größerer Stichprobenumfang wünschenswert gewesen. Hier waren schulorganisatorische Entscheidungen ausschlaggebend. Durch den formalen Rahmen einer schulinternen Evaluation der beiden Gymnasien war die Auswahl an Kursen auf diese beiden Schulen beschränkt.

\section{Interne Validität:}

- Das Design der Studie war quasiexperimentell. Durch die nicht randomisierte, sondern nur durch die äußeren Umstände erfolgte Zuteilung der Schüler in Kontroll- und Treatmentgruppe wurde ein erheblicher Verlust an interner Validität und ein $\mathrm{Zu}$ wachs an Varianz in Kauf genommen. Eine Intervention in schulische Lernprozesse bietet schon an sich große organisatorische Hürden. Eine Randomisierung der Stichprobe wäre aus Gründen der internen Validität hilfreich gewesen, war schulorganisatorisch aber nicht möglich.

- Prinzipiell erfolgt eine Testung im Feld »Schule« im Einfluss vieler unbekannter Variablen, die nicht in der Studie berücksichtigt werden. Die Konfundierung durch Störvariablen (zum Beispiel Wochentag und Uhrzeit der Unterrichtsstunden, Raumsituation, zeitlicher Zusammenhang zu Klausuren in anderen Fächern, parallel stattfindender Schüleraustausch, ...) sorgte für viel Varianz in den Daten.

- Die Testungen der Kontroll- und der Treatmentgruppe erfolgten nicht synchron, sondern um ein Schuljahr zeitversetzt. Zwischenzeitliche Ereignisse an den Schulen könnten demnach einen systematischen Einfluss auf die Treatmentgruppe gehabt haben. Die Entscheidung für eine asynchrone Testung wurde zugunsten der engen Abstimmung zwischen den teilnehmenden Lehrkräften getroffen, was der internen Validität wiederum zu Gute kommt, sowie aus Praktikabilitätsgründen, um die Experimentalaufbauten für die Kontrollgruppe bei der Produktion der Lernvideos für die Treatmentgruppe zu nutzen.

- Neben der durch das asynchrone Versuchsdesign erzeugten Konfundierung ist ein weiterer Umstand kritisch zu betrachten. Die Lehrer unterrichten zuerst die Kontroll-, dann die Treatmentgruppe. Das bedeutet, dass vor allem durch die drei Lehrer, die beide Gruppen unterrichteten, eine weitere Konfundierung der Effekte durch die stets gleiche Methodenabfolge entstanden sein kann. Abhilfe hätte ein Cross-over-Design geschaffen, welches aus unterrichtspraktischen Gründen verworfen wurde (vgl. Kap. $5.1)$. 
- Des Weiteren wurde bereits darauf hingewiesen, dass der Autor einer der teilnehmenden Lehrkräfte war. Bei allem Bemühen um Durchführungs-, Auswertungs- und Interpretationsobjektivität ist die Identität von Forscher und Versuchsteilnehmer äußerst kritisch zu sehen. Die Entscheidung des Autors, die eigenen Physikkurse an der Studie teilnehmen zu lassen, wurde getroffen, um die Stichprobe nicht noch kleiner werden zu lassen. Die ausbleibende Interaktion von Methode und Lehrer (vgl. Kap. 6.3.3) kann den Kritikpunkt der mangelnden Objektivität in einem gewissen Maß relativieren, ohne ihn jedoch vollständig entkräften zu können.

\section{Auswertungsverfahren}

- Zur Auswertung geclusterter Daten, wie sie in dieser Studie vorliegen, ist die Mehrebenenanalyse das Verfahren der Wahl. In Kap. 5.5 wurde bereits dargelegt, dass hierfür jedoch die Stichprobe wesentlich umfangreicher hätte sein müssen.

- Eine Rasch-Skalierung des Fachwissenstests hätte einen Mehrwert bedeutet, um die Fähigkeiten der Schüler und die Schwierigkeit der einzelnen Teilaufgaben des Tests voneinander trennen zu können, wenngleich eine Skalierung durch das offene Format des Tests problematisch gewesen wäre. Doch die Skalierung des Fachwissenstests nach probabilistischer Testtheorie ist angesichts der großen Varianz, die allein durch das Untersuchungsdesign zustande kommt, kein bedeutend effizienteres Instrument der Varianzaufklärung.

- Alternativ zu den in der Studie angewendeten Varianzanalysen wären Strukturgleichungsmodelle denkbar gewesen, die grundsätzlich einen tieferen Einblick in korrelative Zusammenhänge erlaubt hätten und latente Variablen, etwa die kognitive Aktivierung, hätten berücksichtigen können. Die ANOVAs und MANOVAs, denen zur Auswertung der Vorzug gegeben wurde, beschränken sich hingegen auf reine Gruppen- und Untergruppenvergleiche. Bezüglich der Forschungsfragen und -hypothesen sind diese Auswertungsverfahren jedoch hinreichend geeignet und darüber hinaus in empirischen Bildungsforschung am häufigsten eingesetzt (Keselman et al. 1998).

\subsection{Perspektiven}

In einem letzten Schritt soll nun dargelegt werden, inwiefern sich die Befunde der Studie generalisieren lassen. Wo kann Flipped Classroom in Erwartung ähnlich positiver Ergebnisse eingesetzt werden? Der Überblick über die Forschungslage zum Flipped Classroom (vgl. Kap. 2.4.1) zeigte bereits, dass eine große Diversität bezüglich der Umsetzung der Methode, ihrer fachlichen Ausrichtung, der angesprochenen Zielgruppe und des erzielten Erfolgs herrscht. Verallgemeinernde Aussagen über die Wirkungen der Methode sind deshalb schwierig. Die Frage nach der Verallgemeinerung lässt sich aus unterschiedlichen Perspektiven beantworten. Drei Charakteristika der vorliegenden Studie müssen dabei jedoch berücksichtigt werden. 
1. Der Flipped Classroom in seiner Basisform wurde im Fach Physik eingesetzt. Die Übertragung auf andere Bereiche ist dabei aufgrund der Domänenfreiheit dieser Unterrichtsmethode selbstverständlich möglich, aber ohne fachdidaktische Transformation nicht zielführend.

2. Die vorliegende Studie wurde an zwei bayerischen Gymnasien im Physikunterricht der Oberstufe durchgeführt. Schüler, die in der Sekundarstufe an Physikkursen teilnehmen, haben bereits eine Wahlentscheidung für das Fach Physik getroffen. Die Physikkurse bilden deshalb bereits eine Positivauswahl grundsätzlich stärker motivierter und interessierter Schüler. Die affektiven Lernvoraussetzungen in den Physikkursen der Oberstufe sind deshalb sicher anders als in einer typischen Mittelstufenklasse im Fach Physik.

3. Das primäre Ziel der ausgewählten Unterrichtsreihe war es, den Zuwachs an kognitivem Wissen zu fördern, das in den Abiturprüfungen, die den teilnehmenden Schülern der 11. Jahrgangsstufe bevorstanden, in besonderer Weise benötigt wird. In der Basisform des Flipped Classroom lag daher der Schwerpunkt vor allem auf dem Vermitteln, Sichern, Üben, Anwenden, Vertiefen und Übertragen deklarativen Physikwissens. Da der schulische Physikunterricht auch prozessbezogene Kompetenzen fördern muss, bedarf es kritischer Überprüfung, inwiefern sich im Flipped Classroom auch explizit erkenntnisgewinnende, kommunikative und bewertende Kompetenzen ansprechen lassen.

Mit entsprechender Passung der Lernvideos und der Unterrichtsaktivitäten kann Flipped Classroom grundsätzlich in Physikkursen der differenzierten Oberstufe eingesetzt werden. Überall, wo Physikunterricht auf fortgeschrittenem Niveau in wahldifferenzierten Lerngruppen stattfindet, sind die gleichen Strukturbedingungen wie in dieser Studie vorhanden. Das in allen Bundesländern verbreitete Differenzierungssystem der Oberstufe, also ein Kurssystem mit Wahlpflichtbelegungen, bietet die Voraussetzungen, um die Ergebnisse der Studie bundesweit uneingeschränkt übertragen zu können, vorbehaltlich entsprechender Schulungen der Physiklehrkräfte.

Beim Einsatz in der Mittelstufe stellt sich die Frage, ob die von Physikkursen der Oberstufe verschiedene Motivations- und Interessenslage der Schüler zu anderen Ergebnissen führt. Dazu müsste untersucht werden, inwieweit die motivationale Ausgangslage einen Moderator- oder Mediatoreinfluss auf die Wirkung des Flipped Classroom besitzt. Die vorliegende Studie ging dieser Frage nicht nach. Zudem ist der Anteil der Mädchen in den Physikkursen der Oberstufen durch die Kurswahl generell deutlich geringer als in den Mittelstufenklassen. Hier stellt sich die Frage, ob in der Mittelstufe das Geschlecht ebenfalls keine moderierende Wirkung besitzt. Folgestudien zum Flipped Classroom im Physikunterricht der Mittelstufe könnten hier Klarheit bringen.

Im Physikunterricht der Mittelstufe, vor allem aber im physikalischen Anfangsunterricht, darf der Einsatz des Flipped Classroom nicht ausschließlich in der Funktion des deklarativen 
Wissens stehen. Prozessbezogene Kompetenzen müssen dabei genauso gefördert werden, wobei die experimentellen Kompetenzen eine domänenspezifische Besonderheit im Physikunterricht bilden. Es ist zu überlegen und fachdidaktisch zu untersuchen, wie Schüleraktivierung im Flipped Classroom auch die prozessbezogenen Kompetenzen im Physikunterricht ansprechen kann.

Fachdidaktische Folgeuntersuchungen könnten in einem größeren Umfang auch die Rolle und Einstellungen der Lehrkräfte im Flipped Classroom untersuchen, sowohl durch qualitative Interviews als auch durch quantitative Erhebungen. Möglicherweise gibt es Interaktionen mit Lehrertypen, die unterschiedliche Überzeugungen zum Lehren und Lernen von Physik besitzen (vgl. Lamprecht 2011). Auch könnten Unterrichtsbeobachtungen das Lehrerhandeln im Flipped Classroom in den Blick nehmen und untersuchen, ob und inwieweit die Lehrerunterstützung sich von außen betrachtet qualitativ ändert. Die von den Schülern als unverändert wahrgenommene Lehrerunterstützung stand im Widerspruch zur Ausgangshypothese und konnte nur damit erklärt werden, dass die Lehrer schon im traditionellen fragend-entwickelnden Unterricht ein hohes Maß an Unterstützung zeigten. Systematische Unterrichtsbeobachtungen können hier zu einem besseren Verständnis des Unterstützungsverhaltens der Lehrkräfte im Flipped Classroom führen.

Die Rolle des Experiments zeichnet den Physikunterricht gegenüber anderen Fächern aus. In dieser Studie waren reale Experimente organischer Bestandteil der Lernvideos, so dass von einer authentischen Implementierung physikspezifischer Instruktion im Flipped Classroom gesprochen werden kann. Wenn damit eine notwendige Bedingung schulischen Physikunterrichts erfüllt wurde, bedeutet das noch nicht, dass Lernvideos mit videografierten Realexperimenten hinreichende Instruktionsformen im Physikunterricht sind. Die Rolle des Experiments ist für die Erkenntnisgewinnung sowie das Lernen über das Wesen der Naturwissenschaft von grundlegender Bedeutung (Höttecke \& Rieß 2015), auch wenn der $\mathrm{Zu}$ sammenhang zwischen Einsatz von Unterrichtsexperimenten und Lernerfolg einen empirischen Nachweis schuldig bleibt (Roth et al. 1997). Die Rolle kann nicht allein durch Experimente in Lernvideos ausgefüllt werden. Experimente müssen wortwörtlich begriffen werden können, um ihnen als modellbildendes Kriterium naturwissenschaftlicher Erkenntnis gerecht zu werden. Nicht nur, aber auch aufgrund der essentiellen Notwendigkeit des real erlebbaren und interaktiv zugänglichen Experiments kann der Flipped Classroom im Physikunterricht keine Dauermethode sein. Auch die grundsätzliche didaktische Forderung nach Methodenvielfalt gebietet eine ausgewogene Variation von Unterrichtsmethoden, um der Vielfalt der schulischen Aufgabenstellungen und der Heterogenität der Lernvoraussetzungen der Schüler gerecht zu werden (vgl. Meyer 2016, S. 74f). Nur im Kontext anderer Unterrichtsmethoden ist mit Flipped Classroom guter Physikunterricht möglich. Aber innerhalb der gebotenen Methodenvielfalt ist Flipped Classroom im Physikunterricht der Oberstufe eine Methode, die sich erfolgreich einsetzen lässt, um in besonderer Weise das Fachwissen und in gewissem Maße auch affektive Lernmerkmale zu fördern. 



\section{Tabellenverzeichnis}

Tab. 1. Übersicht über die Lerninhalte der Unterrichtsreihe

Tab. 2. Themen, Dauer und Verantwortung für die Lernvideos für die Treatmentgruppe

Tab. 3. Mögliches Design: Cross-over.

Tab. 4. Verwendetes Studiendesign: Asynchrones Pre-/Post-Design mit Kontrollgruppe .. 43

Tab. 5. Zeitlicher Verlaufsplan der Studie. 46

Tab. 6. Konvergente und diskriminante Validität: Korrelationen des Fachwissenstests (post) mit erhobenen Variablen 53

Tab. 7. Variablen des Pretest-Fragebogens, ihre Quellen und Reliabilitäten 55

Tab. 8. Kurs-/Gruppengrößen sowie Geschlechterverteilung. 61

Tab. 9. Vergleich der Leistungsvariablen der Kontroll- und Treatmentgruppe zum Prezeitpunkt

Tab. 10. Mittelwerte der affektiven Merkmale der Kontroll- und Treatmentgruppe zum Prezeitpunkt

Tab. 11. Vergleich des Lernzuwachses (Hakes g) von Kontroll- und Treatmentgruppe 68

Tab. 12. Korrelationsmatrix der Gain Scores der affektiven Lernmerkmale

Tab. 13. Vergleich der Entwicklungen in den affektiven Lernmerkmalen in EinzelANOVAs.

Tab. 14. Lernzuwachs (Hakes g) in Kontroll- und Treatmentgruppe differenziert nach kategorialem Leistungsniveau.

Tab. 15. ANOVAs für Interaktionen von Methode und Leistungsniveau hinsichtlich des Lernzuwachses

Tab. 16. Einfluss der Unterrichtsmethode und des Leistungsniveaus auf den Lernzuwachs (Hakes g)

Tab. 17. Lernausgangslage differenziert nach Jungen und Mädchen (aufgeführt sind die Fälle, von denen ein vollständiger Datensatz vorlag)

Tab. 18. Lernzuwachs in Kontroll- und Treatmentgruppe differenziert nach Mädchen und Jungen.....

Tab. 19. Einfluss der Unterrichtsmethode und des Geschlechts auf den Lernzuwachs (Hakes g)

Tab. 2o. Interaktionen von Methode und Geschlecht hinsichtlich der affektiven

Lernmerkmale 83 
Tab. 21. Motivationsentwicklung (post - pre) in Kontroll- und Treatmentgruppe differenziert nach Mädchen und Jungen 83

Tab. 22. Regressionsanalyse der Motivationsentwicklung 85

Tab. 23. Einfluss der Unterrichtsmethode und der Lehrkraft auf den Lernzuwachs (Hakes g) .87

Tab. 24. Interaktionen von Methode und Lehrer hinsichtlich der affektiven Lernmerkmale 88

Tab. 25. Vergleich der Änderungen in der Lehrerunterstützung in Kontroll- und Treatmentgruppe

Tab. 26. Vergleich der Änderungen in der Hausaufgabendisziplin in Kontroll- und

Treatmentgruppe

Tab. 27. Vergleich der Entwicklung in der Hausaufgabendauer in Kontroll- und

Treatmentgruppe

Tab. 28. Mittlere Hausaufgabendauer pre/post in Kontroll- und Treatmentgruppe in Minuten

Tab. 29. Entwicklung der Hausaufgabenparameter differenziert nach Mädchen und Jungen in Kontroll- und Treatmentgruppe.

Tab. 3o. Einfluss der Unterrichtsmethode und der Lehrkraft auf die Änderung der Lehrerunterstützung und der Stützfaktoren. .96

Tab. 31. Rückmeldungen der Schüler zum Flipped Classroom (Fragebogen) mit integriertem Säulendiagramm. 100

Tab. 32. Veränderung der Screen Time der Schüler (Zeit vor einem Bildschirm) 100

Tab. 33. Mittlere Häufigkeit der Nutzung der Lernvideos mit integriertem Säulendiagramm. 101

Tab. 34. Verständnis der Lernvideos (Schüleranteile) mit integriertem Säulendiagramm ... 102 Tab. 35. Vergleich der Anzahl der eingesetzten Teilaufgaben in der Unterrichtsreihe 104 


\section{Abbildungsverzeichnis}

Abb. 1. Hierarchische Struktur des Selbstkonzepts (Stiensmeier-Pelster \& Schöne 2008, S. 63)

Abb. 2. Zusammenhang lernrelevanter Unterrichtsgrößen …………………............................. 14

Abb. 3. Basisform des Flipped Classroom ......................................................................................... 18

Abb. 4. Theoretische Ebenen des Flipped Classroom ................................................................... 19

Abb. 5. Kategoriale Doppelfunktion von Lernbegleitern (nach Perkhofer-Czapek \&

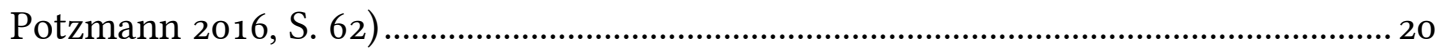

Abb. 6. Beispiel: Versuch aus dem Lernvideo Nr. 8........................................................................ 39

Abb. 7. Beispiel: Herleitung aus dem Lernvideo Nr. 3 ……………………................................... 40

Abb. 8. Beispiel: Sicherungsfolie aus dem Lernvideo Nr. 13 ……............................................ 40

Abb. 9. Abfragegerät CPS-Pulse ${ }^{\mathrm{TM}}$ für das Clicker-Quiz (aus Adidact 2018) ............................. 41

Abb. 10. Beispiel: Folie aus dem Clicker-Quiz zu Lernvideo Nr. 5…………............................... 41

Abb. 11. Basisform des Flipped Classroom als Modell für die Studie............................................ 48

Abb. 12. Flussdiagramm für das Clicker-Quiz ................................................................................ 49

Abb. 13. Vergleich der Eigenschaften der Kontroll- und Treatmentgruppe.................................62

Abb. 14. Boxplots der Physiknoten 11/1 aller Kurse im Fach Physik, Kursbezeichnung: Buchstabe: $\mathrm{K}=$ Kontrollgruppe, $\mathrm{T}$ = Treatmentgruppe; Ziffer: 1-5 Lehrervariable.. 63

Abb. 15. Affektive Lernmerkmale und Stützfaktoren der Kontroll- und Treatmentgruppe zum Prezeitpunkt

Abb. 16. Boxplots der Variable Lehrerunterstützung pre (o-3), Kursbezeichnung: Buchstabe: $\mathrm{K}$ = Kontrollgruppe, $\mathrm{T}$ = Treatmentgruppe; Ziffer: 1-5 Lehrervariable.. 66

Abb. 17. Mittlere Lernzuwächse beider Gruppen (Hakes g) und Boxplots. 68

Abb. 18. Entwicklung der Motivation. 70

Abb. 19. Entwicklung des Fachinteresses im Fach Physik 71

Abb. 2o. Entwicklung des Fachinteresses in den Fächern Mathematik, Chemie, Biologie und Deutsch.

Abb. 21. Entwicklung des physikbezogenen Selbstkonzepts 72

Abb. 22. Lernzuwachs für Schüler im unteren, mittleren und oberen Leistungsniveau 79

Abb. 23. Lernzuwachs (Hakes g) in Kontroll- und Treatmentgruppe differenziert nach Geschlecht 82

Abb. 24. Motivationsentwicklung in Kontroll- und Treatmentgruppe differenziert nach Mädchen und Jungen. 
Abb. 25. Lernzuwachs (Hakes g) nach Lehrkräften ........................................................................... 86

Abb. 26. Entwicklung der Lehrerunterstützung................................................................... 91

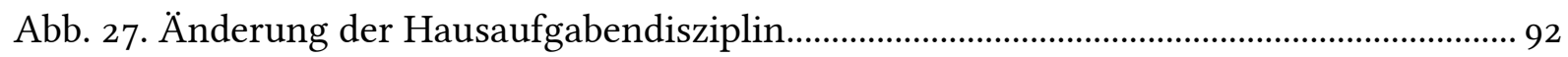

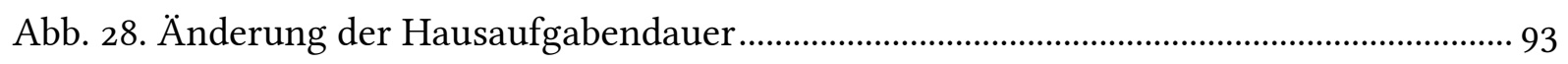

Abb. 29. Änderung der Hausaufgabendisziplin in Kontroll- und Treatmentgruppe differenziert nach Geschlecht ....................................................................................... 95

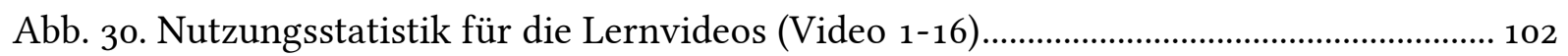

Abb. 31. Verständnisstatistik für die Lernvideos (Video 1-16) unter Angabe des

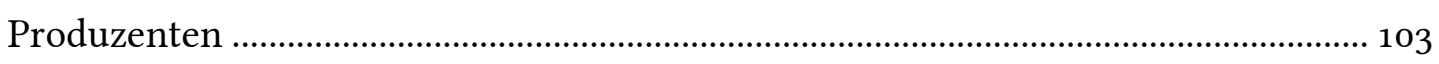




\section{Literaturverzeichnis}

Abeysekera, L. \& Dawson, P., 2015. Motivation and cognitive load in the flipped classroom: definition, rationale and a call for research. Higher Education Research \& Development, 34(1), S. 1-14.

Adidact - Beratung und Training, 2018. CPS-Pulse Flyer. Zugriff am 10.4.2018 auf http://www. adidact.de/PDF/CPS-PULSE_Flyer.pdf

Altemueller, L. \& Lindquist, C., 2017. Flipped classroom instruction for inclusive learning. British fournal of Special Education, 44(3), S. 341-358.

Amabile, T.M. et al., 1994. The Work preference inventory: Assessing intrinsic and extrinsic motivational orientation. Journal of Personality and Social Psychology, 66(5), S. 950-967.

Andrews, T. et al., 2011. Active learning not associated with student learning in a random sample of college biology courses. CBE-Life Sciences Education, 10(4), S. 394-405.

Ash, K., 2012. Educators evaluate flipped classrooms. Education Week, 32(2), S. s6-s8.

Aşıksoy, G. \& Özdaml, F., 2016. Flipped classroom adapted to the ARCS model of motivation and applied to a physics course. Eurasia fournal of Mathematics, Science \& Technology Education, 12(6), S. 1589-1603.

Atwa, Z.M., Din, R. \& Hussin, M., 2017. Effectiveness of flipped learning in physics education on Palestinian high school students' achievement. Fournal of Personalized Learning, 2(1), S. $73-85$.

Baepler, P., Walker, J. \& Driessen, M., 2014. It's not about seat time: Blending, flipping, and efficiency in active learning classrooms. Computers \& Education, 78, S. 227-236.

Bandura, A., 1997. Self-efficacy: The exercise of control, Macmillan.

Bates, S. \& Galloway, R., 2012. The inverted classroom in a large enrolment introductory physics course: a case study. Proceedings of the HEA STEM learning and teaching conference, 1.

Baumeister, R.F. et al., 2003. Does high self-esteem cause better performance, interpersonal success, happiness, or healthier lifestyles? Psychological science in the public interest, 4(1), S. $1-44$.

Bell, M.R., 2015. An investigation of the impact of a flipped classroom instructional approach on high school students' content knowledge and attitudes toward the learning environment, Brigham Young University.

Berger, R. \& Hänze, M., 2004. Das Gruppenpuzzle im Physikunterricht der Sekundarstufe II. Einfluss auf Motivation, Lernen und Leistung. Zeitschrift für Didaktik der Naturwissenschaften, 10, S. 205-219. 
Bergmann, J., 2014. Flipped-learning toolkit: Flipping the non-flippable classes, Zugriff am 10.4.2018 auf https://www.edutopia.org/blog/flipping-the-non-flippable-classes-jonbergmann.

Bergmann, J. \& Sams, A., 2012. Flip your classroom: Reach every student in every class every day, International Society for Technology in Education.

Bergmann, J. \& Sams, A., 2014. Flipping for Mastery. Educational Leadership, 71(4), S. 24-29.

Bhagat, K.K., Chang, C.-N. \& Chang, C.-Y., 2016. The impact of the flipped classroom on mathematics concept learning in high school. Educational Technology \& Society, 19(3), S. $134-142$.

Bidwell, A., 2014. Flipped classroom may help weaker STEM students, Zugriff am 10.4.2018 auf https://www.usnews.com/news/stem-solutions/articles/2014/o8/o5/taking-a-pagefrom-humanities-college-engineering-gets-flipped.

Bishop, J.L., 2013. A controlled study of the flipped classroom with numerical methods for engineers, Utah State University. ProQuest Dissertations Publishing, 3606852.

Bishop, J.L. \& Verleger, M.A., 2013. The flipped classroom: A survey of the research. ASEE National Conference Proceedings, Atlanta, GA, 30(9), S. 1-18.

Bloom, B.S., 1968. Learning for Mastery. Instruction and Curriculum. Regional Education Laboratory for the Carolinas and Virginia, Topical Papers and Reprints, Number 1. Evaluation Comment, 1(2), S. 2.

Bormann, J., 2014. Affordances of flipped learning and its effects on student engagement and achievement, University of Northern Iowa. Zugriff am 10.4.2018 auf https://pdfs. semanticscholar.org/f813/d76360937858e808ef9028c4709eegb68dge.pdf.

Brame, C.J., 2013. Flipping the classroom, Vanderbilt University Center for Teaching. Zugriff am 10.04.2018 auf https://cft.vanderbilt.edu/guides-sub-pages/flipping-the-classroom.

Braun, I. et al., 2012. Inverted Classroom an der Hochschule Karlsruhe - ein nicht quantisierter Flip. In J. Handte \& A. Sperl, eds. Das Inverted Classroom Model: Begleitband zur ersten deutschen ICM-Konferenz. arXiv:1209.4451v1: Oldenbourg Verlag.

BStMUK, Bayerisches Staatsministerium für Unterricht und Kultus, 2004. Lehrplan Physik (G8), Zugriff am 10.4.2018 auf http://www.isb-gym8-lehrplan.de/contentserv/ 3.1.neu/ g8.de/index.php?StoryID=27147.

BStMUK, Bayerisches Staatsministerium für Unterricht und Kultus, 2015. LehrplanPLUS, Zugriff am 10.4.2018 auf http://www.lehrplanplus.bayern.de/bildungs-und-erziehungs auftrag/gymnasium.

BStMUK, Bayerisches Staatsministerium für Unterricht und Kultus, 2018. MEBIS, Zugriff am 10.4.2018 auf https://www.mebis.bayern.de/ueber-mebis/.

Buff, A., 2001. Warum lernen Schülerinnen und Schüler? Eine explorative Studie zur Lernmotivation auf der Basis qualitativer Daten. Zeitschrift für Entwicklungspsychologie und Pädagogische Psychologie, 33(3), S. 157-164. 
Butt, A., 2014. Student views on the use of a flipped classroom approach: Evidence from Australia. Business Education \& Accreditation, 6(1), S. 33.

Casasola, T. et al., 2017. Can flipping the classroom work? Evidence from undergraduate chemistry. International fournal of Teaching and Learning in Higher Education, 29(3), S. $421-435$.

Christoph, G. et al., 2015. Adolescents' computer performance: The role of self-concept and motivational aspects. Computers \& Education, 81, S. 1-12.

Cieliebak, M., 2014. Einfluss von Flipped Classroom auf Fachwissen und Kompetenzen von Studierenden. Zusammenfassung einer Studie der Pädagogischen Hochschule Zürich. Zugriff am 10.04.2018 auf http://dreamboxx.com/mark/data/ZHAW_FlippedClassroom 15.pdf.

Clark, K.R., 2015. The effects of the flipped model of instruction on student engagement and performance in the secondary mathematics classroom. Fournal of Educators Online, 12(1), S. 91-115.

Clark, R. et al., 2006. Efficiency in learning: Evidence-based guidelines to manage cognitive load. Performance Improvement, 45(9), S. 46-47.

Clark, R.E., 1994. Media will never influence learning. Educational technology research and development, 42(2), S. 21-29.

Clark, R.E., 1983. Reconsidering research on learning from media. Review of Educational Research, 53(4), S. 445-459.

Clark, R.E. \& Feldon, D.F., 2014. Ten common but questionable principles of multimedia learning. In R. E. Mayer, ed. The Cambridge Handbook of Multimedia Learning. Cambridge University Press, S. 151-173.

Cohen, J., 1988. Statistical power analysis for the behavioral sciences, Lawrence Earlbaum Associates.

Cornelius-White, J., 2007. Learner-centered teacher-student relationships are effective: A meta-analysis. Review of Educational Research, 77(1), S. 113-143.

Csikszentmihalyi, M. \& Wong, M.M., 2014. Motivation and academic achievement: The effects of personality traits and the quality of experience. In M. Csikszentmihalyi, ed. Applications of flow in human development and education. Springer, S. 437-465.

Daniels, Z., 2008. Entwicklung schulischer Interessen im fugendalter, Waxman.

Day, J.A. \& Foley, J.D., 2006. Evaluating a web lecture intervention in a human - computer interaction course. IEEE Transactions on Education, 49(4), S. 420-431.

Deci, E. \& Ryan, R., 2002. Handbook of Self-Determination Research. In E. Deci \& R. Ryan, eds. University of Rochester Press, S. 3-33. 
Demo, D.H. \& Savin-Williams, R.C., 1992. Self-concept stability and change during adolescence. In R. P. Lipka \& T. M. Brinthaupt, eds. Self-concept stability and change during adolescence. State University of New York Press, S. 116-148.

Demski, J., 2013. 6 Expert Tips for Flipping the Classroom. Campus Technology, 26(5), S. $32-37$.

Deslauriers, L., Schelew, E. \& Wieman, C., 2011. Improved learning in a large-enrollment physics class. science, 332(6031), S. 862-864.

Dimitrov, D.M. \& Rumrill Jr, P.D., 2003. Pretest-posttest designs and measurement of change. Work, 20(2), S. 159-165.

Döring, N. \& Bortz, J., 2016. Forschungsmethoden und Evaluation, Springer.

Douglas, S.S. et al., 2017. Do-it-yourself whiteboard-style physics video lectures. The Physics Teacher, 55(1), S. 22-24.

Eid, M., Gollwitzer, M. \& Schmitt, M., 2010. Statistik und Forschungsmethoden, Beltz.

Enfield, J., 2013. Looking at the impact of the flipped classroom model of instruction on undergraduate multimedia students at CSUN. TechTrends, 57(6), S. 14-27.

Estes, M.D., Ingram, R. \& Liu, J.C., 2014. A review of flipped classroom research, practice, and technologies. International HETL Review, 4(7), S. 1-8.

Fahland, B. et al., 2012. Arbeitspapier Lernbegleitung., Landesinstitut für Lehrerbildung und Schulentwicklung Hamburg. Zugriff am 10.4.2018 auf http://li.hamburg.de/contentblob/ 3546232/data/pdf-grundlagenpapier-lernbegleitung.pdf.

Fähnrich, F. \& Thein, C., 2018. Flip the classroom, Zugriff am 10.4.2018 auf http://www. fliptheclassroom.de/.

Fautch, J.M., 2015. The flipped classroom for teaching organic chemistry in small classes: is it effective? Chemistry Education Research and Practice, 16(1), S. 179-186.

Field, A., 2013. Discovering Statistics using IBM SPSS, Sage.

Findlay-Thompson, S. \& Mombourquette, P., 2014. Evaluation of a flipped classroom in an undergraduate business course. Business Education \& Accreditation, 6(1), S. 63-71.

Flipped Learning Network, 2014. Definition of Flipped Learning, Zugriff am 10.4.2018 auf https://flippedlearning.org/definition-of-flipped-learning/.

Foldnes, N., 2016. The flipped classroom and cooperative learning: Evidence from a randomised experiment. Active Learning in Higher Education, 17(1), S. 39-49.

Fryling, M., Yoder, R. \& Breimer, E., 2016. Full flip, half flip and no flip: Evaluation of flipping an introductory programming course. Information Systems Education fournal, 14(5), S.4.

Fulton, K., 2012. Upside down and inside out: Flip your classroom to improve student learning. Technological Horizons In Education, 39(8), S. 12-17. 
Galway, L.P. et al., 2014. A novel integration of online and flipped classroom instructional models in public health higher education. BMC medical education, 14(1), S. 181.

Gilboy, M.B., Heinerichs, S. \& Pazzaglia, G., 2015. Enhancing student engagement using the flipped classroom. Fournal of Nutrition Education and Behavior, 47(1), S. 109-114.

Gillette, C. et al., 2018. A systematic review and meta analysis of student pharmacist outcomes comparing flipped classroom and lecture. American fournal of Pharmaceutical Education, e-View. https://doi.org/10.5688/ajpe6898.

Goerres, A., Kärger, C. \& Lambach, D., 2015. Aktives Lernen in der Massenveranstaltung: Flipped-Classroom-Lehre als Alternative zur klassischen Vorlesung in der Politikwissenschaft. Zeitschrift für Politikwissenschaft, 25(1), S. 135-152.

González-Gómez, D. et al., 2016. Performance and Perception in the Flipped Learning Model: An Initial Approach to Evaluate the Effectiveness of a New Teaching Methodology in a General Science Classroom. Journal of Science Education and Technology, 25(3), S. $450-459$.

Greenberg, B., Medlock, L. \& Stephens, D., 2011. Blend my learning: Lessons learned from a blended learning pilot, Zugriff am 10.4.2018 auf https://blendmylearning.files.wordpress. com/2011/12/lessons-learned-from-a-blended-learning-pilot4.pdf.

Gregorius, R.M., 2017. Performance of underprepared students in traditional versus animation-based flipped-classroom settings. Chemistry Education Research and Practice, 18(4), S. $841-848$.

Grehn, J. \& Krause, J., 2009. Physik Bayern 11, Schroedel.

Gudjons, H., 2014. Handlungsorientiert lehren und lernen: Schüleraktivierung - SelbständigkeitProjektarbeit, Klinkhardt.

Guo, P.J., Kim, J. \& Rubin, R., 2014. How video production affects student engagement: An empirical study of MOOC videos. Proceedings of the first ACM conference on Learning@ scale, S. 41-50.

Hake, R.R., 1998. Interactive-engagement versus traditional methods: A six-thousand-student survey of mechanics test data for introductory physics courses. American fournal of Physics, 66(1), S. 64-74.

Hannover, B. \& Kessels, U., 2001. Monoedukativer Anfangsunterricht in Physik in der Gesamtschule. Auswirkungen auf Motivation, Selbstkonzept und Einteilung in Grundund Fortgeschrittenenkurse. Zeitschrift für Entwicklungspsychologie und Pädagogische Theorie, 34, S. 201-215.

Hao, Y., 2016. Middle school students' flipped learning readiness in foreign language classrooms: Exploring its relationship with personal characteristics and individual circumstances. Computers in Human Behavior, 59, S. 295-303.

Hasselhorn, M. \& Gold, A., 2017. Pädagogische Psychologie, Kohlhammer.

Hattie, J., Beywl \& Zierer, K., 2015. Lernen sichtbar machen, Schneider. 
Häussler, P. \& Hoffmann, L., 2000. A curricular frame for physics education: Development, comparison with students' interests, and impact on students' achievement and selfconcept. Science Education, 84(6), S. 689-705.

Häussler, P. \& Hoffmann, L., 1998. Chancengleichheit für Mädchen im Physikunterricht. Ergebnisse eines erweiterten BLK-Modellversuchs. Zeitschrift für Didaktik der Naturwissenschaften, 1, S. 51-67.

Häussler, P. \& Hoffmann, L., 1995. Physikunterricht - an den Interessen von Mädchen und Jungen orientiert. Unterrichtswissenschaft, 23(2), S. 107-126.

Helmke, A., 1992. Selbstvertrauen und schulische Leistungen, Hogrefe.

Herbst, M., Fürtbauer, E.M. \& Strahl, A., 2016. Interesse an Physik - in Salzburg. PhyDid BDidaktik der Physik-Beiträge zur DPG-Frühjahrstagung.

Herreid, C.F. \& Schiller, N.A., 2013. Case studies and the flipped classroom. Fournal of College Science Teaching, 42(5), S. 62-66.

Herrmann-Rottmair, F. et al., 2009. Physik Bayern Gymnasium - Lehrbuch für die Klasse 11, Duden Schulbuch.

Herwartz-Emden, L., 2007. Neues aus alten Schulen - empirische Studien in Mädchenschulen, Budrich.

Herzog, W., 1996. Motivation und naturwissenschaftliche Bildung. Kriterien eines »mädchengerechten « koedukativen Unterrichts. Neue Sammlung, 36, S. 61-91.

Hidi, S., 200o. Chapter 11 - An interest researcher's perspective: The effects of extrinsic and intrinsic factors on motivation. In C. Sansone \& J. M. Harackiewicz, eds. Intrinsic and Extrinsic Motivation. Educational Psychology. San Diego: Academic Press, S. 309-339.

Hidi, S. \& Renninger, K.A., 2006. The four-phase model of interest development. Educational psychologist, 41(2), S. 111-127.

Hirsch, J., 2005. Interesse, Handlungen und situatives Erleben von Schülerinnen und Schülern beim Bearbeiten physikalischer Aufgaben, Logos.

Hoffmann, L., 2002. Promoting girls' interest and achievement in physics classes for beginners. Learning and instruction, 12(4), S. 447-465.

Hoffmann, L., Häußler, P. \& Lehrke, M., 1998. Die IPN-Interessenstudie, IPN.

Höttecke, D. \& Rieß, F., 2015. Naturwissenschaftliches Experimentieren im Lichte der jüngeren Wissenschaftsforschung - Auf der Suche nach einem authentischen Experimentbegriff der Fachdidaktik. Zeitschrift für Didaktik der Naturwissenschaften, 21(1), S. 127-139.

IES, Institute of Education Sciences, 2018. ERIC. Zugriff am 10.4.2018 auf https://eric.ed. gov/?q=flipped+classroom+physics. 
ISB, Staatsinstitut für Schulqualität und Bildungsforschung, 2010. Interne Evaluation an Bayerns Schulen. Konzeptionelle Grundlagen, Anregungen und Vorstellung eines Instrumentariums für einen zentralen Bereich der Schulentwicklung, Bayerisches Staatsministerium für Unterricht und Kultus. Zugriff am 10.4.2018 auf http://www.interne-evaluation.isb.bayern.de/download/15o/interne_evaluation_2010_ online.pdf.

Izard, C.E. et al., 200o. Self-organization of discrete emotions, emotion patterns, and emotioncognition relations. In M. D. Lewis \& I. Granic, eds. Emotion, development, and selforganization: Dynamic systems approaches to emotional development. Cambridge University Press, S. 15-36.

Jensen, J.L., Kummer, T.A. \& Godoy, P.D. d M., 2015. Improvements from a flipped classroom may simply be the fruits of active learning. CBE-Life Sciences Education, 14(1), S. $1-12$.

Johnson, L. \& Renner, J., 2012. Effect of the flipped classroom model on secondary computer applications course: student and teacher perceptions, questions and student achievement, University of Louisville, KY.

Keller, G. \& Thiel, R.-D., 1998. Lern- und Arbeitsverhaltensinventar (LAVI), Hogrefe.

Kenner, A. \& Jahn, D., 2016. Flipped Classroom - Hochschullehre und Tutorien umgedreht gedacht. In A. Eßer, H. Kröpke, \& H. Wittau, eds. Tutorienarbeit im Diskurs III Qualifizierung für die Zukunft. WTM, S. 35-58.

Keselman, H. et al., 1998. Statistical practices of educational researchers: An analysis of their ANOVA, MANOVA, and ANCOVA analyses. Review of Educational Research, 68(3), S. $350-386$.

Kiselka, A., 2016. NEUroLOGISCH: Inverted Classroom ermöglicht individuelles und praxisbezogenes Lernen, FFH Open Access Repository - Forschungsforum der österreichischen Fachhochschulen. Zugriff am 10.4.2018 auf http://ffhoarep.fh-ooe.at/ handle/123456789/662.

Kleickmann, T., 2011. Was passiert mit dem Interesse an Physik im Übergang von der Primarin die Sekundarstufe? In D. Kucharz, T. Irion, \& B. Reinhoffer, eds. Grundlegende Bildung ohne Brüche. Springer, S. 219-222.

Klieme, E., Rakoczy, K. \& others, 2008. Empirische Unterrichtsforschung und Fachdidaktik. Outcome-orientierte Messung und Prozessqualität des Unterrichts. Zeitschrift für Pädagogik, 54(2), S. 222-237.

Klieme, E., Schümer, G. \& Knoll, S., 2001. Mathematikunterricht in der Sekundarstufe I: »Aufgabenkultur« und Unterrichtsgestaltung. In E. Klieme \& J. Baumert, eds. TIMSSImpulse für Schule und Unterricht. Bundesministerium für Bildung und Forschung, S. $43-57$.

Klippert, H., 2008. Besser lernen: Kompetenzvermittlung und Schüleraktivierung im Schulalltag, Klippert Verlag. 
Köller, O. et al., 2006. Zum Zusammenspiel von schulischer Leistung, Selbstkonzept und Interesse in der gymnasialen Oberstufe. Zeitschrift für pädagogische Psychologie, 20(1/2), S. $27-39$.

Krapp, A., 2003. Die Bedeutung der Lernmotivation für die Optimierung des schulischen Bildungssystems. Politische Studien, 54(3), S. 91-105.

Krapp, A., 1998. Entwicklung und Förderung von Interessen im Unterricht. Psychologie in Erziehung und Unterricht, 44(3), S. 185-201.

Krapp, A., 2006. Interesse. In D. Rost, ed. Handwörterbuch Pädagogische Psychologie. Beltz, S. $880-886$.

Krapp, A., 1992. Interesse, Lernen und Leistung. Neuere Forschungsansätze in der Pädagogischen Psychologie. Zeitschrift für Pädagogik, 38(5), S. 747-770.

Krapp, A., 2002. Structural and dynamic aspects of interest development: Theoretical considerations from an ontogenetic perspective. Learning and instruction, 12(4), S. $383-409$.

Krapp, A. \& Prenzel, M., 2011. Research on interest in science: Theories, methods, and findings. International journal of science education, 33(1), S. 27-50.

Krapp, A., Schiefele, U. \& Schreyer, I., 1993. Metaanalyse des Zusammenhangs von Interesse und schulischer Leistung. Zeitschrift für Entwicklungspsychologie und pädagogische Psychologie, 10(2), S. 120-148.

Kück, A., 2014. Unterrichten mit dem Flipped Classroom-Konzept: Das Handbuch für individualisiertes und selbstständiges Lernen mit neuen Medien, Verlag an der Ruhr.

Kuhn, J., 2014. Mehrebenenanalyse am Beispiel der Lernwirkung von Aufgaben. In D. Krüger, I. Parchmann, \& H. Schecker, eds. Methoden in der naturwissenschaftsdidaktischen Forschung. Springer, S. 297-310.

Kunter, M., 2005. Multiple Ziele im Mathematikunterricht, Waxmann.

Kunter, M. \& Voss, T., 2011. Das Modell der Unterrichtsqualität in COACTIV: Eine multikriteriale Analyse. In M. Kunter et al., eds. Forschung zur professionellen Kompetenz von Lehrkräften - Ergebnisse des Projekts. Waxmann, S. 85-113.

Lage, M.J., Platt, G.J. \& Treglia, M., 200o. Inverting the classroom: A gateway to creating an inclusive learning environment. The fournal of Economic Education, 31(1), S. 30-43.

Lamprecht, J., 2011. Ausbildungswege und Komponenten professioneller Handlungskompetenz, Logos.

Lape, N.K. et al., 2014. Probing the inverted classroom: A controlled study of teaching and learning outcomes in undergraduate engineering and mathematics. 121st ASEE Annual Conference \& Expositions, Paper ID 9475.

Lee, G. \& Wallace, A., 2018. Flipped learning in the English as a foreign language classroom: Outcomes and perceptions. TESOL Quarterly, 52(1), S. 62-84. 
Lehmann, K. et al., 2015. Flipping the Classroom-IT-unterstützte Lerneraktivierung zur Verbesserung des Lernerfolges einer universitären Massenlehrveranstaltung. HMD Praxis der Wirtschaftsinformatik, 52(1), S. 81-95.

Leibniz-Institut für Wissenschaftsmedien, 2017. Inverted Classroom, Zugriff am 10.4.2018 auf https://www.e-teaching.org/lehrszenarien/vorlesung/inverted_classroom.

Lewalter \& Schreyer, I., 200o. Entwicklung von Interessen und Abneigungen - zwei Seiten einer Medaille? Studie zur Entwicklung berufsbezogener Abneigungen in der Erstausbildung. In U. Schiefele \& K.-P. Wild, eds. Interesse und Lernmotivation: Untersuchungen zu Entwicklung, Förderung und Wirkung. Waxmann, S. 11-30.

Lewis, C.E., Chen, D.C. \& Relan, A., 2018. Implementation of a flipped classroom approach to promote active learning in the third-year surgery clerkship. The American fournal of Surgery, 215(2), S. 298-303.

Lin, P.-C. \& Chen, H.-M., 2016. The effects of flipped classroom on learning effectiveness: using learning satisfaction as the mediator. World Transactions on Engineering and Technology Education, 14(2), S. 231-244.

Lipowsky, F., 2009. Unterricht. In E. Wild \& J. Möller, eds. Pädagogische Psychologie. Springer, S. $73^{-101 .}$

Lo, C.K. \& Hew, K.F., 2017. Using »First Principles of Instructions « to design secondary school mathematics flipped classroom: The findings of two exploratory studies. Educational Technology \& Society, 20(1), S. 222-236.

Love, B. et al., 2014. Student learning and perceptions in a flipped linear algebra course. International fournal of Mathematical Education in Science and Technology, 45(3), S. $317-324$.

Lyman, F.T., 1981. The responsive classroom discussion: The inclusion of all students. In A. S. Anderson, ed. Mainstreaming digest. University of Maryland, S. 109-113.

MacKinnon, G., 2015. Determining useful tools for the flipped science education classroom. Contemporary Issues in Technology and Teacher Education, 15(1), S. 44-55.

Marcey, D.J. \& Brint, M.E., 2012. Transforming an undergraduate introductory biology course through cinematic lectures and inverted classes: A preliminary assessment of the clic model of the flipped classroom. Biology Education Research Symposium at the Meeting of the National Association of Biology Teachers, 12, S. 24.

Markus, H. \& Kunda, Z., 1986. Stability and malleability of the self-concept. fournal of Personality and Social Psychology, 51(4), S. 858.

Marlowe, C., 2012. The effect of the flipped classroom on student achievement and stress, Montana State University.

Marsh, H.W. et al., 2005. Academic self-concept, interest, grades, and standardized test scores: Reciprocal effects models of causal ordering. Child development, 76(2), S. 397-416. 
Marsh, H.W. \& Craven, R.G., 2006. Reciprocal effects of self-concept and performance from a multidimensional perspective: Beyond seductive pleasure and unidimensional perspectives. Perspectives on Psychological Science, 1(2), S. 133-163.

Martin, A.P., 2018. LEIFI Physik. Available at: https://www.leifiphysik.de/bayern/11-klasse.

Mayer \& Chandler, P., 2001. When learning is just a click away: Does simple user interaction foster deeper understanding of multimedia messages? Journal of Educational Psycho$\log y, 93(2)$, S. 390-397.

Mayer, R.E., 2014. Cognitive theory of multimedia learning. In R. E. Mayer, ed. The Cambridge Handbook of Multimedia Learning. Cambridge University Press, S. 43-71.

Mazur, E., 2014. Peer instruction: A user's manual, Pearson.

McLaughlin, J.E. et al., 2014. The flipped classroom: a course redesign to foster learning and engagement in a health professions school. Academic Medicine, 89(2), S. 236-243.

Meyer, H., 2002. Einführung in die Schulpädagogik. In H. Kiper, H. Meyer, \& W. Topsch, eds. Einführung in die Schulpädagogik. Cornelson Scriptor, S. 109-121.

Meyer, H., 2016. Was ist guter Unterricht?, Cornelson Scriptor.

Missildine, K. et al., 2013. Flipping the classroom to improve student performance and satisfaction. Fournal of Nursing Education, 52(10), S. 597-599.

Mitchell, M., 1993. Situational interest: Its multifaceted structure in the secondary school mathematics classroom. Fournal of Educational Psychology, 85(3), S. 424-436.

Möller, J. \& Trautwein, U., 2009. Selbstkonzept. In E. Wild \& M. J., eds. Pädagogische Psychologie. Springer, S. 179-203.

Munson, A. \& Pierce, R., 2015. Flipping content to improve student examination performance in a pharmacogenomics course. American journal of pharmaceutical education, 79(7), S. 103.

Neumann \& Borowski, A., 2010. Naturwissenschaftliche Bildung als Beitrag zur Gestaltung partizipativer Demokratie. In D. Höttecke, ed. Naturwissenschaftliche Bildung als Beitrag zur Gestaltung partizipativer Demokratie. LIT.

Neumann, K., Kauertz, A. \& Fischer, H.E., 2012. Quality of instruction in science education. In B. Fraser, K. Tobin, \& C. J. McRobbie, eds. Second International Handbook of Science Education. Springer, S. 247-258.

Nicolaisen, T., 2016. Unterstützung von Lernprozessen in Schule und Unternehmen. In R. Wegener et al., eds. Coaching als individuelle Antwort auf gesellschaftliche Entwicklungen. Springer, S. 113-120.

Nimmerfroh, M.-C., 2016. Flipped Classroom. Der DIE-Wissensbaustein für die Praxis, Zugriff am 10.4.2018 auf http://www.die-bonn.de/wb/2016-flipped-classroom-01.pdf. 
O'Flaherty, J. \& Phillips, C., 2015. The use of flipped classrooms in higher education: A scoping review. The Internet and Higher Education, 25, S. 85-95.

OECD, Organisation for Economic Co-operation \& Development, 2016. PISA 2015 Assessment and Analytical Framework: Science, Reading, Mathematic and Financial Literacy, OECD publishing.

Oser, F.K. \& Baeriswyl, F.J., 2001. Choreographies of teaching: Bridging instruction to learning. In E. Richardson, ed. Handbook of Reseach on Teaching. AERA, S. 1031-1065.

Pearson Education, 2013. Flipped learning model dramatically improves course pass rate for atrisk students, Zugriff am 10.4.2018 auf http://assets.pearsonschool.com/asset_mgr/ current/201317/Clintondale_casestudy.pdf.

Pekrun, R., 1988. Emotion, Motivation und Persönlichkeit, Psychologie-Verlag-Union.

Pekrun, R. \& Schiefele, U., 1996. Emotions-und motivationspsychologische Bedingungen der Lernleistung. In F. E. Weinert, ed. Psychologie des Lernens und der Instruktion. S. $153-180$.

Perkhofer-Czapek, M. \& Potzmann, R., 2016. Begleiten, Beraten und Coachen, Springer.

Perselli, V., 2016. Flipped Classroom im Fremdsprachenunterricht - Möglichkeiten und Herausforderungen, Linköpings Universitet.

Pierce, R. \& Fox, J., 2012. Vodcasts and active-learning exercises in a »flipped classroom «? Model of a renal pharmacotherapy module. American fournal of Pharmaceutical Education, 76(10), S. 196.

Prenzel, M. et al., 2007. PISA 2006: Die Ergebnisse der dritten internationalen Vergleichsstudie, Waxmann.

Puentedura, R., 2006. Transformation, technology, and education, Zugriff am 10.4.2018 auf http://www.hippasus.com/resources/tte/puentedura_tte.pdf.

Rahman, A. et al., 2014. The influences of flipped classroom: A meta analysis. In ICEED, 6th International Conference on Engineering Education. IEEE Conference Publications, S. $24-28$.

Rakoczy, K., Klieme, E. \& Pauli, C., 2008. Die Bedeutung der wahrgenommenen Unterstützung motivationsrelevanter Bedürfnisse und des Alltagsbezugs im Mathematikunterricht für die selbstbestimmte Motivation. Zeitschrift für pädagogische Psychologie, 22(1), S. 25-35.

Robinson, S. et al., 2015. Effectiveness of flipped classroom techniques in an advanced laboratory physics course. In M. Eblen-Zayas, E. Behringer, \& J. Kozminski, eds. Proceedings of the Conference on Laboratory Instruction BFY 2015. DOI: 10.1119/ bfy.2015.pr.023, S. 92-95.

Roehl, A., Reddy, S.L. \& Shannon, G.J., 2013. The flipped classroom: An opportunity to engage millennial students through active learning. Fournal of Family and Consumer Sciences, 105(2), S. 44 . 
Rost, D.H. \& Sparfeldt, J.R., 2002. Facetten des schulischen Selbstkonzepts. Ein Verfahren zur Messung des differentiellen Selbstkonzepts schulischer Leistungen und Fähigkeiten (DISK-Gitter). Diagnostica, 48(3), S. 130-140.

Rost, D.H., Sparfeldt, J.R. \& Schilling, S.R., 2006. Hochbegabung. In K. Schweizer, ed. Leistung und Leistungsdiagnostik. Springer, S. 187-222.

Roth, W.-M. et al., 1997. Why may students fail to learn from demonstrations? A social practice perspective on learning in physics. Journal of Research in Science Teaching, 34(5), S. 509-533.

Ryan, M.D. \& Reid, S.A., 2015. Impact of the flipped classroom on student performance and retention: A parallel controlled study in general chemistry. Fournal of Chemical Education, 93(1), S. 13-23.

Sadaghiani, H.R., 2012. Online prelectures: An alternative to textbook reading assignments. The Physics Teacher, 50(5), S. 301-303.

Said, M.N.H.M. \& Zainal, R., 2017. A review of impacts and challenges of flipped-mastery classroom. Advanced Science Letters, 23(8), S. 7763-7766.

Schiefele, U., 2008. Lernmotivation und Interesse. In W. Schneider \& M. Hasselhorn, eds. Handbuch der Pädagogischen Psychologie. Hogrefe, S. 38-49.

Schiefele, U., 2009. Motivation. In E. Wild \& J. Möller, eds. Pädagogische Psychologie. Springer, S. $151-177$.

Schiefele, U. \& Schreyer, I., 1994. Intrinsische Lernmotivation und Lernen. Ein Überblick zu Ergebnissen der Forschung. Zeitschrift für Pädagogische Psychologie, 8(1), S. 1-13.

Schiepe-Tiska, A. et al., 2016. PISA 2015. Eine Studie zwischen Kontinuität und Innovation. In K. Reiss et al., eds. PISA. Waxmann, S. 45-98.

Schilling, S.R., Sparfeldt, J.R. \& Rost, D.H., 2006. Facetten schulischen Selbstkonzepts: Welchen Unterschied macht das Geschlecht? Zeitschrift für Pädagogische Psychologie, $20(1 / 2)$, S. $9-18$.

Schmidt, S., 2018. Mein Flipped Classroom, Zugriff am 10.4.2018 auf https://www.flippedmathe. de/mein-flipped-classroom.

Schnirch, A., 2006. Gendergerechte Interessen- und Motivationsförderung im Kontext naturwissenschaftlicher Grundbildung - Konzeption, Entwicklung und Evaluation einer multimedial unterstützten Lernumgebung, Logos.

Schraw, G. \& Dennison, R.S., 1994. The effect of reader purpose on interest and recall. fournal of Reading Behavior, 26(1), S. 1-18.

Schultz, D. et al., 2014. Effects of the flipped classroom model on student performance for advanced placement high school chemistry students. Journal of Chemical Education, 91(9), S. 1334-1339. 
Şengel, E., 2016. To FLIP or not to FLIP: Comparative case study in higher education in Turkey. Computers in Human Behavior, 64, S. 547-555.

Shavelson, R.J., Hubner, J.J. \& Stanton, G.C., 1976. Self-concept: Validation of construct interpretations. Review of Educational Research, 46(3), S. 407-441.

Van Sickle, J., 2016. Discrepancies between Student Perception and Achievement of Learning Outcomes in a Flipped Classroom. Journal of the Scholarship of Teaching and Learning, 16(2), S. 29-38.

Smith, M.K. et al., 2009. Why peer discussion improves student performance on in-class concept questions. Science, 323(5910), S. 122-124.

Souvignier, E. \& Gold, A., 2006. Wirksamkeit von Lehrmethoden. In K. Schweizer, ed. Leistung und Leistungsdiagnostik. Springer, S. 146-166.

Spannagel, C., 2017. Flip your class!, Zugriff am 10.4.2018 auf http://flipyourclass.christianspannagel.de.

Spannagel, C. \& Freisleben-Teutscher, C.F., 2016. Inverted Classroom meets Kompetenzorientierung. Beiträge zum 5. Tag der Lehre an der FH St. Pölten am 2o. Oktober 2016, Zugriff am 10.4.2018 auf http://skill.fhstp.ac.at/wp-content/uploads/2016/10/ spannagel _freisleben_kompetenz.pdf.

Spiel, C., Wagner, P. \& Fellner, G., 2002. Wie lange arbeiten Kinder zu Hause für die Schule? Eine Analyse in Gymnasium und Grundschule. Zeitschrift für Entwicklungspsychologie und Pädagogische Psychologie, 34(3), S. 125-135.

Sterner, E. et al., 2017. Evaluating the flipped classroom approach in engineering education: Students' attitudes, engagement and performance in an undergraduate sustainability course. In 45th SEFI Conference, 18-21 September 2017, Azores, Portugal. S. 1-8.

Stiensmeier-Pelster, J. \& Schöne, C., 2008. Fähigkeitsselbstkonzept. In W. Schneider \& M. Hasselhorn, eds. Handbuch der Pädagogischen Psychologie. Hogrefe, S. 62-73.

Stoll, S., 2018. 180Grad-Flip, Zugriff am 10.4.2018 auf https://www.180grad-flip.de.

Sun, J.C.-Y. \& Wu, Y.-T., 2016. Analysis of learning achievement and teacher-student interactions in flipped and conventional classrooms. The International Review of Research in Open and Distributed Learning, 17(1), S. 79-99.

Sun, J.C.-Y., Wu, Y.-T. \& Lee, W.-I., 2017. The effect of the flipped classroom approach to OpenCourseWare instruction on students' self-regulation. British fournal of Educational Technology, 48(3), S. 713-729.

Sun, Z., Xie, K. \& Anderman, L.H., 2018. The role of self-regulated learning in students' success in flipped undergraduate math courses. The Internet and Higher Education, 36, S. $41-53$.

Sweller, J., 1994. Cognitive load theory, learning difficulty, and instructional design. Learning and instruction, 4(4), S. 295-312. 
Tent, L., 2006. Zensuren. In D. Rost, ed. Handwörterbuch pädagogische Psychologie. Beltz, S. $873-880$.

Tesch, M. \& Duit, R., 2004. Experimentieren im Physikunterricht - Ergebnisse einer Videostudie. Zeitschrift für Didaktik der Naturwissenschaften, 10(10), S. 51-69.

Thai, N.T.T., De Wever, B. \& Valcke, M., 2017. The impact of a flipped classroom design on learning performance in higher education: Looking for the best »blend « of lectures and guiding questions with feedback. Computers \& Education, 107, S. 113-126.

Tobias, S., 1994. Interest, prior knowledge, and learning. Review of Educational Research, 64(1), S. $37-54$.

Tolks, D. et al., 2016. Eine Einführung in die Inverted/Flipped-Classroom-Methode in der Ausund Weiterbildung in der Medizin und den Gesundheitsberufen. GMS fournal for Medical Education, 33(3), S. 12-23.

Trautwein, U. et al., 2006. Self-esteem, academic self-concept, and achievement: how the learning environment moderates the dynamics of self-concept. Fournal of Personality and Social Psychology, 9o(2), S. 334-349.

Valentine, DuBois \& Cooper, H., 2004. The relation between self-beliefs and academic achievement: A meta-analytic review. Educational Psychologist, 39(2), S. 111-133.

Wagner, P. \& Spiel, C., 2002. Zeitinvestment und Lerneffektivität: Eine Analyse in Hauptschule und Gymnasium hinsichtlich Persönlichkeitsvariablen, Arbeitshaltung und Bedingungsfaktoren. Empirische Pädagogik, 16(3), S. 357-381.

Walper, L.M., 2017. Entwicklung der physikbezogenen Interessen und selbstbezogenen Kognitionen von Schülerinnen und Schülern in der Übergangsphase von der Primar-in die Sekundarstufe. Eine Längsschnittanalyse vom vierten bis zum siebten Schuljahr, Logos.

Weber, T., 2003. Kumulatives Lernen im Physikunterricht. Eine vergleichende Untersuchung in Unterrichtsgängen zur geometrischen Optik, Logos.

Webster, D.R., Majerich, D.M. \& Madden, A.G., 2016. Flippin’ Fluid Mechanics - Comparison Using Two Groups. Advances in Engineering Education, 5(3), S. 1-20.

Wild, E. \& Hofer, M., 200o. Elterliche Erziehung und die Veränderung motivationaler Orientierungen in der gymnasialen Oberstufe und der Berufsschule. In U. Schiefele \& K.-P. Wild, eds. Interesse und Lernmotivation: Untersuchungen zu Entwicklung, Förderung und Wirkung. Waxmann, S. 31-52.

Wild, E. \& Möller, J., 2009. Pädagogische Psychologie, Springer.

Winkelmann, J., 2013. Auswirkungen auf den Fachwissenszuwachs und auf affektive Schülermerkmale durch Schüler- und Demonstrationsexperimente im Physikunterricht, Logos.

Winter, J.B., 2013. The effect of the flipped classroom model on achievement in an introductory college physics course. Mississippi State University. 
Wodzinski, R., 2007. Physikdidaktik in der Praxis. In E. Kircher \& W. Schneider, eds. Physikdidaktik in der Praxis. Springer, S. 559-580.

Wolters, C.A. \& Pintrich, P.R., 1998. Contextual differences in student motivation and selfregulated learning in mathematics, English, and social studies classrooms. Instructional Science, 26(1), S. 27-47.

Wong, K. \& Chu, D.W., 2014. Is the Flipped Classroom Model Effective in the Perspectives of Students' Perceptions and Benefits? In S. K. S. Cheung et al., eds. Hybrid Learning. Theory and Practice. ICHL 2014. Lecture Notes in Computer Science, vol 8595. S. 93-104, DOI: $10.1007 / 978-3-319-08961-4 \_10$.

Wylie, R.C., 1979. The self concept: Theory and research on selected topics, vol 2, University of Nebraska Press.

Yıldrım, F.S. \& Kıray, S.A., 2016. Flipped classroom model in education. In W. Wu, S. Alan, \& M. T. Hebebci, eds. Research Highlights in Education and Science 2016. S. 2-8.

Zahay, D., Kumar, A. \& Trimble, C., 2017. Motivation and active learning to improve student performance. In M. Stieler, ed. Creating Marketing Magic and Innovative Future Marketing Trends. Developments in Marketing Science: Proceedings of the Academy of Marketing Science. Springer, S. 1259-1263, DOI: 10.1007/978-3-319-45596-9_231.

Zainuddin, Z. \& Halili, S.H., 2016. Flipped classroom research and trends from different fields of study. The International Review of Research in Open and Distributed Learning, 17(3), S. $313-340$.

Zainuddin, Z. \& Perera, C.J., 2017. Exploring students' competence, autonomy and relatedness in the flipped classroom pedagogical model. Fournal of Further and Higher Education, DOI: $10.1080 / 0309877 X .2017 .1356916$.

Zheng, W., Becker, T. \& Ding, X., 2014. The Effects of »Flipped Classroom« Concept on the Effectiveness of Teaching. Proceedings of 2014 of the ASEE North Midwest Section Conference, DOI: 10.17077/aseenmw2014.1019.

Ziegenspeck, J. \& Lehmann, J., 1999, Handbuch Zensur und Zeugnis in der Schule: historischer Rückblick, allgemeine Problematik, empirische Befunde und bildungspolitische Implikationen, Klinkhardt.

Zimmerman, B.J., 200o. Self-efficacy: An essential motive to learn. Contemporary Educational Psychology, 25(1), S. 82-91.

Zoe, H. et al., 2014. Role of face-to-face lecturing in large enrollment physics classes. arXiv preprint arXiv:1404.3566. 



\section{Anhang}

Anhang 1a. Zeitlicher Ablaufplan der Studie (Kontrollgruppe 2015/16)

(in Klammern: Einzel- oder Doppelstunde)

\begin{tabular}{|c|c|c|}
\hline Datum & Woche & Stundeninhalte \\
\hline Mo (2), 7.3 & & Pretest \\
\hline $\mathrm{Do}(1), 10 \cdot 3$ & & Puffer (Nachschriften) \\
\hline Mo (2), $14 \cdot 3$ & 1 & $\begin{array}{l}\text { Phänomen Induktion und Anwendungen, Leiterschaukel im Hufeisen- } \\
\text { magneten, Herleitung von } \bigcup_{i}=\text { IvB }\end{array}$ \\
\hline \multirow[t]{2}{*}{ Do (1), 17.3. } & & Magnet in Spule, Vertiefung \\
\hline & & Osterferien \\
\hline Mo (2), 4.4 & 2 & $\begin{array}{l}\text { All. Induktionsgesetz, } U_{i}=-N \text { dot Phi, Feld- und Flächenänderung, } \\
\text { Beispiele }\end{array}$ \\
\hline Do (1), 7.4 . & & Drehender Leiterrahmen im B-Feld, Transformator \\
\hline Mo (2), 11.4 & 3 & $\begin{array}{l}\text { Lenzsche Regel: Ring und Stabmagnet, Kraftaufwand bei kurzgeschlos- } \\
\text { senem Handgenerator }\end{array}$ \\
\hline Do (1), 14.4 & & Thomson-Ringversuch \\
\hline Mo (2), 18.4 & 4 & $\begin{array}{l}\text { Wechselspannung, Generator mit Messgerät, Herleitung von } \\
U_{i}=-N 2 \pi f A B \cdot \sin (2 \pi f t)\end{array}$ \\
\hline Do (1), 21.4 & & Effektivwerte, $U_{\text {eff }}=U_{\max } /$ sqrt 2 \\
\hline Mo (2), 25.4 & 5 & Vertiefung Wechselspannung, Selbstinduktion (Lampen an Spule) \\
\hline Do (1), 28.4 & & Einschaltvorgang einer Spule (Cobra), Diagramme \\
\hline Mo (2), 2.5 & 6 & Einschaltvorgang mit $U_{i}=-L$ dot I, Emag $=1 / 2 L^{2}$ \\
\hline Do, 5.5 & & Christi Himmelfahrt \\
\hline Mo (2), 9.5 & 7 & Vertiefung Selbstinduktion; Induktionsherd \\
\hline \multirow[t]{2}{*}{ Do (1), 12.5} & & Schwingkreis \\
\hline & & Pfingstferien \\
\hline Mo (2), 30.5 & 8 & Schwingkreis, Herleitung Thomsonformel \\
\hline Do (1), 2.6. & & Erzwungene Schwingung und Resonanz \\
\hline Mo (2), 6.6 & & Posttest \\
\hline
\end{tabular}

$=23$ Stunden 
Anhang 1b. Zeitlicher Ablaufplan der Studie (Treatmentgruppe 2016/17)

(in Klammern: Einzel- oder Doppelstunde)

\begin{tabular}{|c|c|c|}
\hline Datum & Woche & Stundeninhalte \\
\hline Di, 7.2. & & Pretest \\
\hline Do, 9.2. (1) & & Puffer (Nachschriften) \\
\hline $\mathrm{Di}, 14.2 .(2)$ & 1 & Einführung \\
\hline Do, 16.2. (1) & & Induktion durch Flächenänderung \\
\hline $\mathrm{Di}, 21.2 .(2)$ & 2 & Induktion durch Magnetfeldänderung \\
\hline \multirow[t]{2}{*}{ Do, 23.2. (1) } & & Lenzsche Regel \\
\hline & & Faschingsferien \\
\hline $\mathrm{Di}, 7 \cdot 3 \cdot(2)$ & 3 & Wirksame Fläche \\
\hline Do, 9.3. (1) & & - ausgefallen - \\
\hline $\mathrm{Di}, 14 \cdot 3 \cdot(2)$ & 4 & Transformator und Induktionsherd \\
\hline Do, 16.3. (1) & & Erzeugung von Wechselspannung \\
\hline $\mathrm{Di}, 21.3 .(2)$ & 5 & Effektivwerte \\
\hline Do, 23.3. (1) & & Selbstinduktion \\
\hline $\mathrm{Di}, 28.3 .(2)$ & 6 & Ein- und Ausschaltvorgang \\
\hline Do, 30.3. (1) & & Magnetische Energie \\
\hline $\mathrm{Di}, 4.4 .(2)$ & 7 & Anwendung der Selbstinduktion \\
\hline \multirow[t]{2}{*}{ Do, 6.4. (1) } & & Schwingkreis Einführung \\
\hline & & Osterferien \\
\hline Di, 25.4. (2) & 8 & Ungedämpfter Schwingkreis und Energien \\
\hline Do, 27.4. (1) & & Thomson \\
\hline $\mathrm{Di}, 2.5 .(2)$ & & Erzwungene Schwingung und Resonanz \\
\hline Do, 4.5. (1) & & Puffer \\
\hline Di 9.5. & & Posttest \\
\hline
\end{tabular}

$=25$ Stunden 
Anhang 2a. Durchführungserläuterung des Pretests

1. Vorabinformation:

Bitte vorlesen:

»Der Test besteht aus zwei Teilen, einem Fragbogenteil (15 min) und einem Leistungstest (max. $70 \mathrm{~min}$ ). Bitte lest jede Frage des Fragebogens sorgfältig durch und beantwortet sie so genau wie möglich. In diesem Fragebogen gibt es keine richtigen oder falschen Antworten. Gebt die Antworten, die ihr für richtig halten. Eure Angaben können nicht mit eurer Person in Verbindung gebracht werden. Deshalb schreibt auch bitte nicht euren Namen auf die Bögen. Die Fragebögen aller drei Kurse werden sofort gemischt.

Um den individuellen Lernzuwachs während der Unterrichtsreihe abbilden zu können, bedarf es einer Pseudonymisierung des Erhebungsbogens. Damit kann der Vor- und der Nachtest der gleichen Person zugeordnet werden.

Um einen Teilnehmercode zu erstellen, verfahrt bitte so, wie ihr es jetzt in diesem Beispiel seht.«

(Schülern die Folie zur Generierung der TN-ID zeigen)

2. Dann Fragebogen austeilen. Für das Ausfüllen ist dann 15 min Zeit.

Bitte folgende Hinweise vorlesen:

»Bitte verwendet Kugelschreiber o.̈̈.. Keinen Bleistift und keinen zu starken Filzstift.«

"Wenn ihr etwas korrigieren müsst, müsst ihr das Feld ganz ausfüllen und das Kreuz woanders setzen «

»Randmarkierungen nicht überschreiben.«

3. Nach dem Fragebogen dann diese Informationen zum Leistungstest vorlesen:

»Der Leistungstest, der nun kommt, will euer Vorwissen abfragen und überprüfen, was ihr zu diesem Thema schon könnt. Vieles werdet ihr noch nicht kennen. Es ist ganz normal, wenn ihr viele Aufgaben noch nicht lösen könnt. Es soll gewissermaßen eure individuelle Ausgangsbasis erhoben werden.

Am Ende der Unterrichtsreihe im funi werdet ihr einen ähnlichen Leistungstest bekommen, den ihr dann sicher besser lösen könnt. So zeigt sich dann euere Leistungssteigerung am besten.

Für den Leistungstest dürft ihr einen Taschenrechner, aber keine Formelsammlung verwenden."

- Bögen mit den eingelegten Tests austeilen.

- Wenn ihn alle haben, Teilnehmer-ID oben rechts auf den Rand und das Datum eintragen lassen. Dann öffnen und bearbeiten. Dafür ist 70 min (exakt) Bearbeitungszeit.

- Währenddessen können die ausgefüllten Fragebögen eingesammelt werden. 
Anhang 2b. Durchführungserläuterung des Posttests

1. Vorabinformation:

Bitte vorlesen:

»Der Test besteht wieder aus zwei Teilen, einem Fragbogenteil (ca. 8 min) und einem Leistungstest (7o min). Bitte lest jede Frage des Fragebogens sorgfältig durch und beantwortet sie so genau wie möglich.

Um den individuellen Lernzuwachs während der Unterrichtsreihe abbilden zu können, benötigt ihr aus Gründen der Pseudonymisierung den gleichen Teilnehmercode, den ihr damals beim ersten Test erstellt habt. Erstellt ihn deshalb bitte wieder genau nach folgendem Muster.«

(Schülern die Folie zur Generierung der TN-ID zeigen)

2. Dann den Fragebogen austeilen. Für das Ausfüllen sind dann 5-8 min Zeit.

Bitte folgende Hinweise vorlesen:

»Bitte verwendet Kugelschreiber oder ähnliches, keinen Bleistift und keinen zu starken Filzstift. Wenn ihr etwas korrigieren müsst, müsst ihr das Feld ganz ausfüllen und das Kreuz woanders setzen. Bitte Randmarkierungen nicht überschreiben.«

»Wenn im Fragenbogen von, den letzten zwei Monaten' die Rede ist, ist die gesamte Unterrichtsreihe, Induktion und elektromagnetischer Schwingkreis ' mit der Flipped Classroom Methode gemeint.«

3. Nach dem Fragebogen dann diese Informationen zum Leistungstest vorlesen:

»Der Leistungstest, der nun kommt, will überprüfen, welche Fähigkeiten ihr zum Thema Induktion und Schwingkreis erworben habt. Für den Leistungstest dürft ihr einen Taschenrechner, aber keine Formelsammlung verwenden.«

Bögen mit den eingelegten Tests austeilen.

»Bitte schreibt oben rechts auf den Rand eure Teilnehmer-ID und das heutige Datum."

Wenn alle die Eintragung gemacht haben:

»Ihr könnt jetzt anfangen. Ihr habt exakt 7o Minuten Zeit.«

Währenddessen können die ausgefüllten Fragebögen eingesammelt werden. 
Anhang 3a. Fachwissenstest

c01pre2016

2. Teil - Leistungstest

TN-ID:

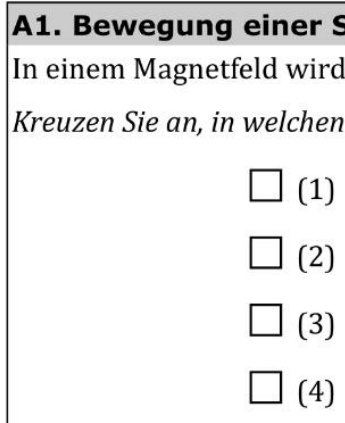

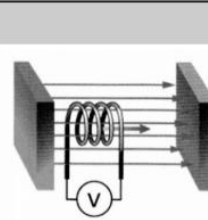

(1)

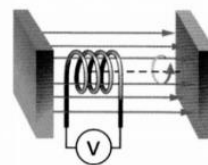

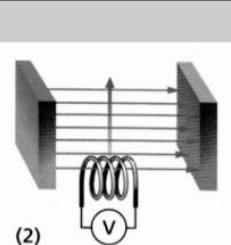

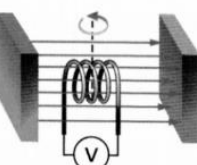

(4)

\section{A2. Generator}

Eine Leiterschleife dreht sich im Uhrzeigersinn im Magnetfeld zwischen den Polen eines Magneten.
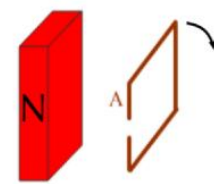

1
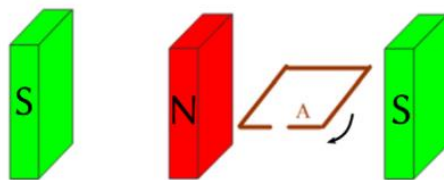

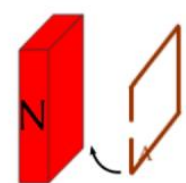

3

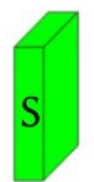

S

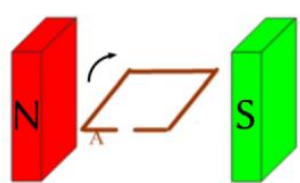

4

Kreuzen Sie alle wahren Aussagen an.

$\square$ Die Spannung ist zu den Zeitpunkten 1 und 3 maximal.

$\square_{3}$ Im Zeitpunkt 1 ist A der Pluspol.

Die Spannung ist zu den Zeitpunkten 2 und 4 maximal.

$\square$ Im Zeitpunkt 2 ist A der Pluspol.

$\square$ Im Zeitpunkt 3 ist A der Pluspol.

$\square_{6}$ Im Zeitpunkt 4 ist A der Pluspol.

\section{A3. Leiterrahmen im Magnetfeld}

Ein geschlossener quadratischer Leiterrahmen mit der Kantenlänge $l=10 \mathrm{~cm}$ besitzt den ohmschen Widerstand $\mathrm{R}=0,3 \Omega$ und eine Masse von $\mathrm{m}=7 \mathrm{~g}$. Oberhalb eines scharf begrenzten Magnetfelds der Flussdichte B =1,2 T wird er losgelassen.

Solange der Leiterrahmen nicht vollständig in das Feld eingetaucht ist, fällt er nach einer kurzen Beschleunigungsphase mit einer konstanten Geschwindigkeit nach unten.

a) Begründen Sie ohne Rechnung, weshalb ein Induktionsstrom im Leiterrahmen genau nur dann fließt, während er in das Magnetfeld ein-bzw. aus ihm austritt.

b) Erklären Sie, warum die Fallgeschwindigkeit beim Eintritt einen konstanten Wert annimmt.

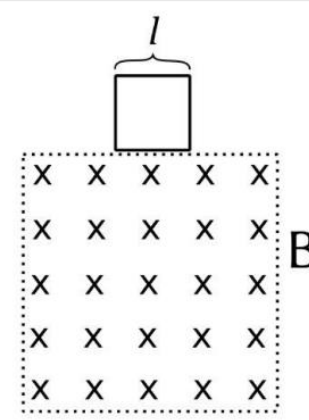

c) Zeigen Sie, dass für die konstante Geschwindigkeit in der Eintrittsphase gilt: $v=\frac{m g R}{l^{2} B^{2}}$.

Berechnen Sie den anschließend den Wert der Fallgeschwindigkeit.

\section{A4. Strom in einer Spule}

In einer luftgefüllten Spule der Länge $l=70 \mathrm{~cm}$, der Windungszahl $\mathrm{N}=500$ und $12 \mathrm{~cm}$ Durchmesser wird die Stromstärke in der Zeit $\Delta \mathrm{t}=4 \mathrm{~s}$ von $\mathrm{I}_{1}=12 \mathrm{~A}$ auf $\mathrm{I}_{2}=1 \mathrm{~A}$ reduziert. $\left[\mu_{0}=4 \pi \cdot 10^{-7} \mathrm{Vs} / \mathrm{Am}\right]$

a) Berechnen Sie die Induktivität der Spule.

b) Berechnen Sie die Selbstinduktionsspannung während des Stromabfalls. 


\section{A5. Einschaltvorgang in einer Spule}

Eine reale Spule wird an eine Spannungsquelle angeschlossen. Beim Einschaltvorgang wird die Stromstärke in Abhängigkeit von der Zeit aufgenommen. Es wird eine Messung mit Eisenkern und eine ohne Eisenkern durchgeführt. Man erhält die beiden Messkurven im unten stehenden Diagramm.
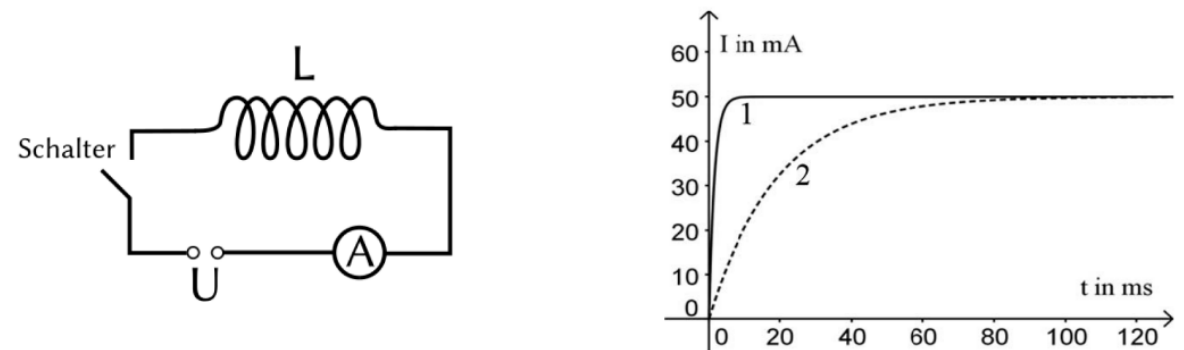

a) Erklären Sie, weshalb in beiden Fällen die Stromstärke nicht sofort ihren Maximalwert erreicht. Entscheiden und begründen Sie, bei welcher Messung der Eisenkern verwendet wurde.

b) Begründen Sie (stichpunktartig), warum sich in beiden Fällen nach einiger Zeit die gleiche Stromstärke einstellt.

\section{A6. Schwingkreis mit Lautsprecher}

Ein elektromagnetischer Schwingkreis, bestehend aus einer Spule mit Eisenkern der Induktivität $\mathrm{L}=0,25 \mathrm{H}$ und einem Kondensator der Kapazität $\mathrm{C}=0,15 \mu \mathrm{F}$, schwingt ungedämpft mit seiner Eigenfrequenz f. Als Nachweisgerät der Schwingung dient ein Lautsprecher.

a) Berechnen Sie die Frequenz $f$ des vom Lautsprecher abgegebenen Tons.

b) Erläutern und begründen Sie (stichpunktartig), wie sich die Tonhöhe verhält, wenn man den Eisenkern langsam aus der Spule herauszieht.

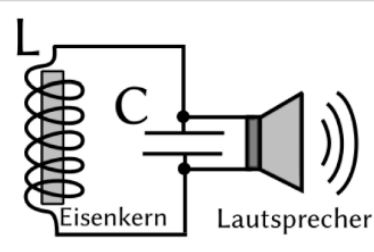

Der Eisenkern befindet vollständig in der Spule. Zum Zeitpunkt $t=0$ ist der Kondensator mit einer Spannung von $12 \mathrm{~V}$ maximal geladen.

c) Bestimmen Sie die Maximalstromstärke I im Schwingkreis. [zur Kontrolle: 9,3 mA]

d) Nach $t=0,3 \mathrm{~ms}$ ist der Kondensator das erste Mal vollständig entladen. Zeichnen Sie den zeitlichen Verlauf der Spannung und der Stromstärke innerhalb einer Schwingungsdauer in die beiden Koordinatensysteme.
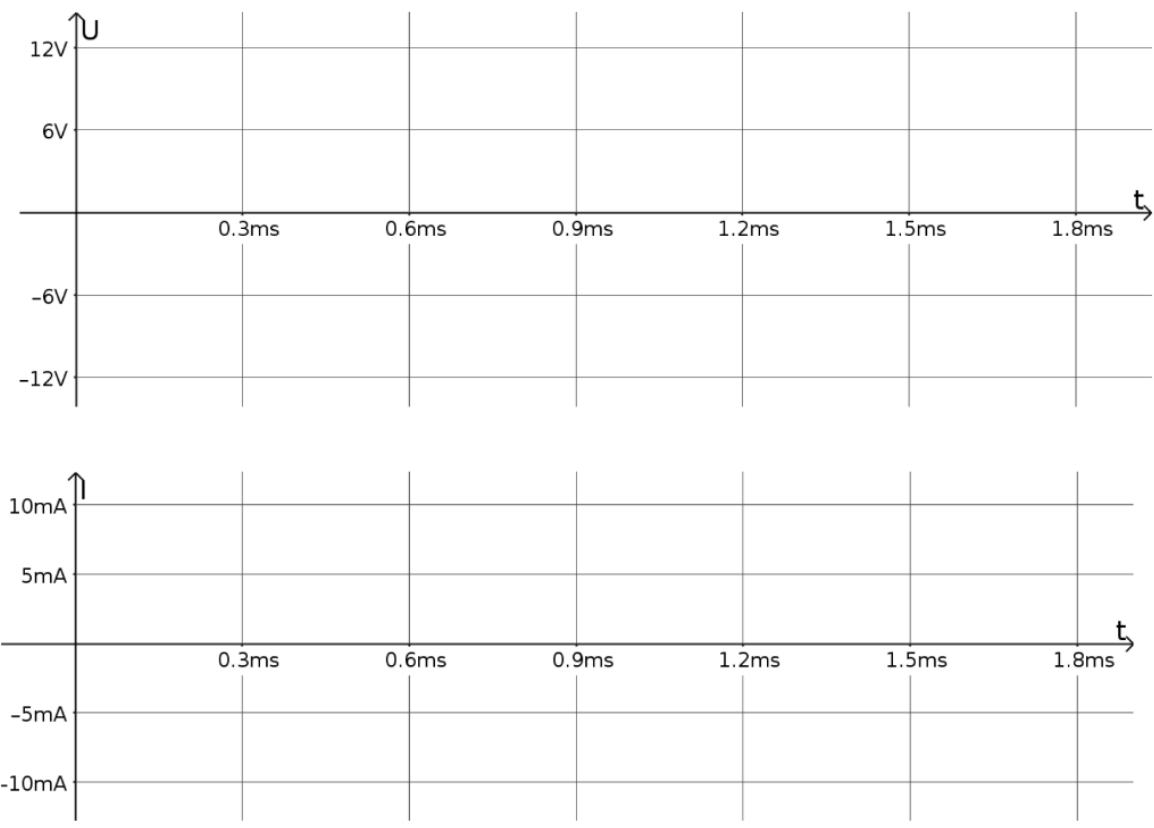
Anhang 3b. Quellen der Aufgaben des Fachwissenstests

A1 | Physik Gymnasium 11. Bayern. Berlin: Duden Paetec 2009, S. 138, 2 a

A2 | leifiphysik.de [www.leifiphysik.de/elektrizitaetslehre/elektromagnetische-induktion/aufgaben\#LEIFI-Quiz]

A3 | 'Induktion' (Abi 2004 GPh1, 2) und 'Induktion' (Abi 2002 GPh1, 2)

A4 | Metzler Physik 11, Ausgabe Bayern. Braunschweig: Schroedel 2009, S89, 1, leicht verändert

A5 | 'Spulenexperimente' (Abi $2011 \mathrm{Ph}$ 11-1, 3a+b), leicht verändert

A6 | 'Schwingkreis' (Abi 2004 GPh2, 1) und 'Spulenexperimente' (Abi 2011 Ph 11-1, 3e), teilweise verändert 
Anhang 4. Fragebogen Pretest Seite 1

\begin{tabular}{|c|cc|c|}
\hline EvaSys & 1. Teil - Fragebogen & t03pre2017 & Electric Paper \\
\hline \hline Fakultät für Physik und Astronomie & Frank Finkenberg & \\
Lehrstuhl für Physik und ihre Didaktik & Flipped Classroom & \\
& & \\
& & \\
& &
\end{tabular}

Bitte so markieren: $\square \bigotimes \square \square \square$ Bitte verwenden Sie einen Kugelschreiber oder nicht zu starken Filzstift. Dieser Fragebogen wird maschinell erfasst. Korrektur: $\quad \square \square \square \bigotimes \square$ Bitte beachten Sie im Interesse einer optimalen Datenerfassung die links gegebenen Hinweise beim Ausfüllen.

1. Teilnehmerkennung

1.1 Nennen Sie hier Ihre TN-ID:

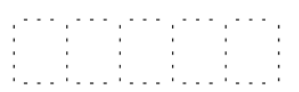

\section{Allgemeine Angaben}

2.1 Sind Sie weiblich oder männlich?

2.2 Welche Schule besuchen Sie?

2.3 Welche Sprache sprechen Sie am häufigsten zuhause?

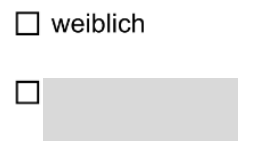

$\square$ Deutsch

$\square$ Spanisch,

Französisch,

Italienisch,

Portugisisch

Englisch $\square$ Russisch

Polnisch,

Serbisch,

Tschechisch

$\square$ andere

2.4 Haben Sie schon einmal eine Klasse wiederholt?

$\square$ Nein, nie

$\square$ Ja, einmal

$\square$ Ja, zweimal oder öfter

3. Wie sehr stimmen Sie folgenden Aussagen über sich selbst zu?

3.1 Ich arbeite lieber in der Gruppe als allein.

3.2 Ich kann gut zuhören.

3.3 Ich nehme Rücksicht auf die Interessen der anderen.

3.4 Ich finde, dass Gruppen bessere Entscheidungen fällen als Einzelpersonen.

3.5 Ich finde, dass sich beim Arbeiten in der Gruppe auch meine persönliche Leistung steigert.

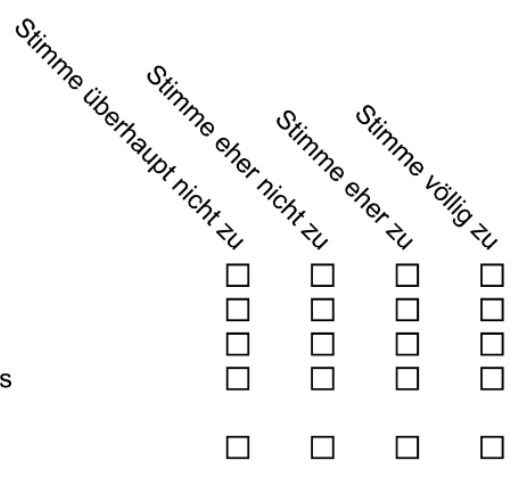


Anhang 4. Fragebogen Pretest Seite 2

EvaSys 1. Teil - Fragebogen

t03pre2017

4. Wie sehr stimmen Sie folgenden Aussagen in Bezug auf Ihre Person zu?

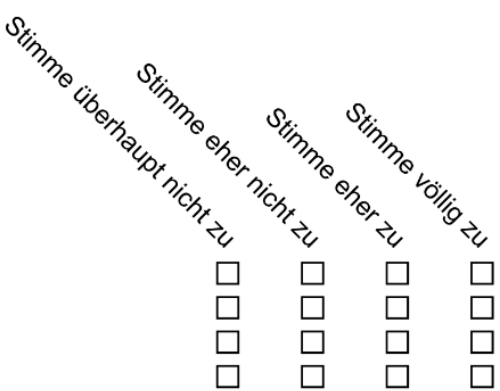

4.1 Ich möchte in möglichst allen Fächern Bestnoten erzielen.

4.2 Ich möchte in allem, was ich tue, der/die Beste sein.

4.3 Ich sehe mich selber als ehrgeizige Person.

4.4 Ich möchte einer/eine der Besten in meinem Kurs sein.

5. In welchem Umfang stimmen Sie folgenden Aussagen über sich selbst zu?

5.1 Ich lasse Dinge gern auf mich zukommen.

5.2 Ich mag es, wenn alles bei mir nach einem Plan läuft.

5.3 Ich mache mir in allen Fächern gut organisierte Mitschriften.

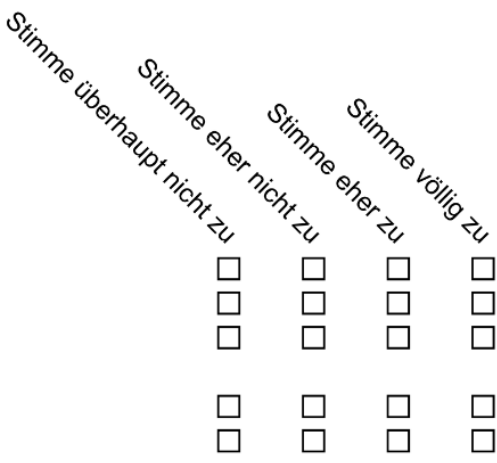

5.5 Ich mache oft detailierte to-do-Listen.

6. Wie sehr stimmen Sie folgenden Aussagen in Bezug auf Ihre Person zu?

6.1 Ich schiebe Hausaufgaben oft vor mir her.

6.2 Wenn ich mich auf einen Test oder eine Klausur vorbereite,

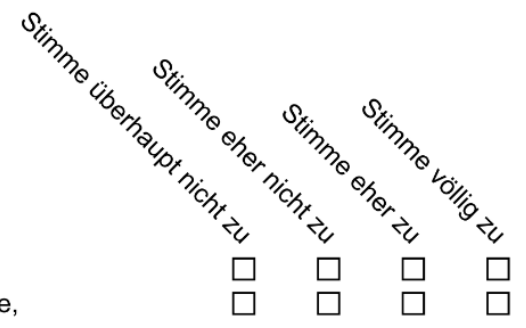
verschwende ich oft Zeit mit anderen Dingen.

6.3 Ich habe meine Aufgaben oft früher als nötig fertig.

6.4 Ich erledige Dinge oft in letzter Minute.

6.5 Es kommt vor, dass ich Dinge mache, die ich schon seit Tagen erledigen wollte. 
Anhang 4. Fragebogen Pretest Seite 3

\begin{tabular}{|l|ll|l}
\hline EvaSys & 1. Teil - Fragebogen & t03pre2017 Electric Paper \\
\hline
\end{tabular}

7. Wie sehr stimmen Sie folgenden Aussagen zu?

7.1 Meine Eltern interessieren sich für meine schulischen

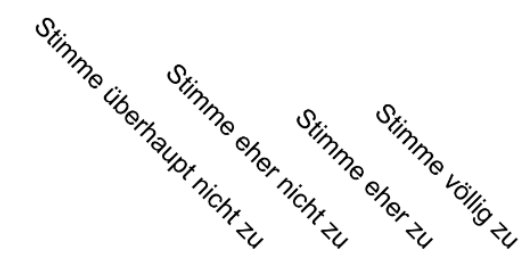
Aktivitäten.

7.2 Meine Eltern unterstützen meine schulischen Anstrengungen und Erfolge.

7.3 Meine Eltern unterstützen mich, wenn ich Schwierigkeiten in der Schule habe.

7.4 Meine Eltern ermutigen mich, auf mich selbst zu vertrauen

8. Wie sehr stimmen Sie folgenden Aussagen in Bezug auf Ihre Person zu?

8.1 Ich glaube, dass ich anspruchsvollen Stoff im

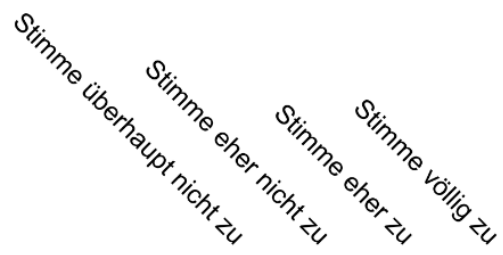
Physikunterricht leicht lernen kann.

8.2 Normalerweise kann ich Fragen im Physikunterricht gut beantworten.

8.3 Ich lerne neuen Stoff im Physikunterricht schnell.

8.4 Den Stoff im Physikunterricht finde ich einfach.

8.5 Es fällt mir leicht, neue Ideen im Physikunterricht zu verstehen.

9. Wie sehr stimmen Sie folgenden Aussagen in Bezug auf Ihre Person zu?

9.1 Im Allgemeinen macht es mir Spaß, mich mit physikalischen Themen zu befassen.

9.2 Ich lese gerne etwas über Physik

9.3 Ich beschäftige mich gerne mit physikalischen Problemen.

9.4 Ich beschäftige mich in meiner Freizeit mit Themen der Physik

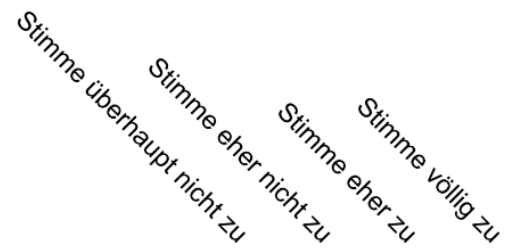


Anhang 4. Fragebogen Pretest Seite 4

\begin{tabular}{l|l} 
EvaSys & 1. Teil - Fragebogen
\end{tabular} t03pre2017

10. Wie sehr stimmen Sie folgenden Aussagen in Bezug auf Ihre Person zu?

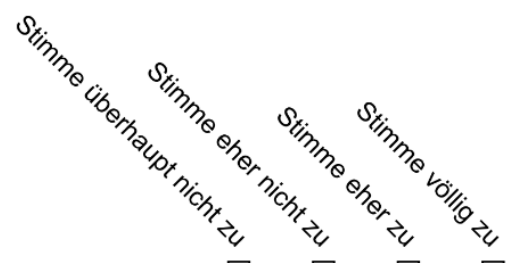

10.1 Nach der Schule würde ich gerne etwas mit Naturwissenschaften studieren.

10.2 Nach der Schule würde ich gerne einen Beruf erlernen, der etwas mit Physik zu tun hat.

10.3 Nach der Schule würde ich gerne einen Beruf erlernen, der etwas mit Technik zu tun hat.

11. Denken Sie an die letzten zwei Monate: Wie sehr waren Sie an den folgenden Schulfächern interessiert?

11.1 Deutsch

11.2 Mathematik

11.3 Chemie

11.4 Physik

11.5 Biologie
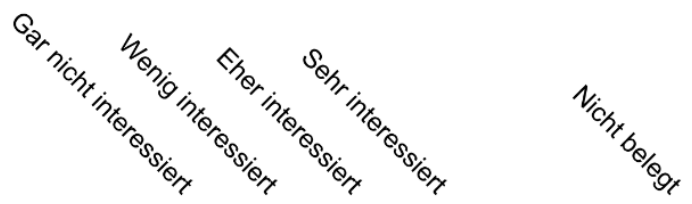

12. Denken Sie an die letzten zwei Monate: Wie oft kam Folgendes in Ihrem Physikunterricht vor?

12.1 Unser Physiklehrer interessiert sich für den Lernfortschritt jedes einzelnen Schülers.

12.2 Unser Physiklehrer unterstützt uns zusätzlich, wenn wir Hilfe brauchen.

12.3 Unser Physiklehrer unterstützt uns beim Lernen.

12.4 Unser Physiklehrer erklärt uns etwas so lange, bis wir es verstehen.

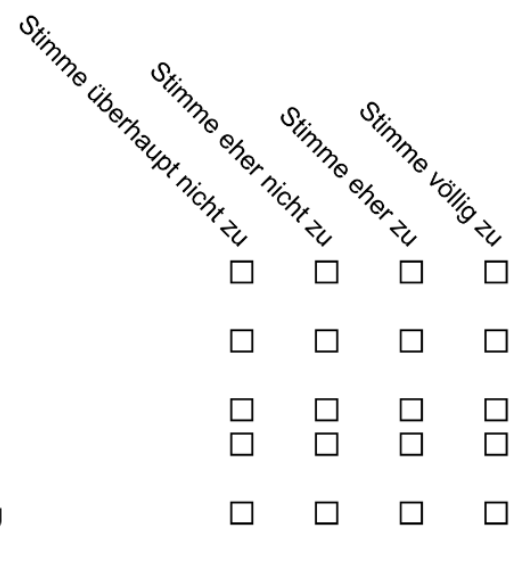


Anhang 4. Fragebogen Pretest Seite 5

13.1 Seien Sie ehrlich: Wie regelmäßig haben Sie in den letzten 2 Monaten die Physikhausaufgaben erledigt?

13.2 Wie viel Zeit haben Sie während der letzten 2 Monate durchschnittlich für eine Physikhausaufgabe investiert?

13.3 Welche Note hatten Sie im Fach Physik im Jahreszeugnis der 10. Klasse?
$\square 5$
$\square 4$
$\square 1$
$\square 3$
$\square$ weiß nicht mehr

13.4 Wie viele Punkte hatten Sie im Fach Physik im Halbjahreszeugnis der 11. Jahrgangsstufe?
$\square 0$
$\square 1$
$\square 2$
$\square 3$
$\square 4$
$\square 5$
$\square 6$
$\square 7$
$\square 8$
$\square 9$
$\square 10$
$\square 11$
$\square 12$
$\square 13$
$\square 14$
$\square 15$
$\square$ weiß nicht mehr

13.5 Wie viele Punkte würden Sie sich im Fach Physik für den Zeitraum der letzten 2 Monate selbst geben?
$\square 0$
$\square 1$
$\square 4$
$\square 7$
$\square 2$
$\square 3$
$\square 7$
$\square 5$
$\square 6$
$\square 10$
$\square 8$
$\square 12$
$\square 13$
$\square 11$
$\square 14$

$\square 15$

13.6 Wie viele Punkte hatten Sie im Fach Mathematik im Halbjahreszeugnis der 11. Jahrgangsstufe?
$\square 0$
$\square 3$
$\square 6$
$\square 1$
$\square 2$
$\square 4$
$\square 7$
$\square 5$
$\square 9$
$\square 10$
$\square 8$
$\square 12$
$\square 13$
$\square 11$
$\square 15$
$\square$ weiß nicht mehr
$\square 14$

13.7 Möchten Sie das Halbjahr Physik 11/2 ins Abitur einbringen?

$\square$ Nein

$\square$ Vielleicht

$\square \mathrm{Ja}$ 
Anhang 4. Fragebogen Pretest Seite 6

EvaSys 1. Teil - Fragebogen

t03pre2017

(2) Electric Paper

14. Wie oft verwenden Sie außerhalb der Schule den Computer (Smartphone, Tablet, PC, Notebook) für folgende Aktivitäten?

14.1 Spiele im Einzelspieler-Modus

14.2 Online-Spiele für mehrere Spieler

14.3 E-Mails lesen und schreiben

14.4 Teilnahme an sozialen Netzwerken (z.B. Facebook, WhatsApp, Twitter,...)

14.5 Zum Vergnügen im Internet surfen (wie etwa zum Videoschauen, z.B. auf YouTube)

14.6 Lesen von Nachrichten im Internet (z.B. Tagesgeschehen)

14.7 Suchen nach praktischen Informationen im Internet (z. B. Ort und Zeit von Veranstaltungen)

14.8 Musik, Filme, Spiele oder Software vom Internet herunterladen

14.9 Ins-Netz-Stellen von eigenen Inhalten (z. B. Musik, Gedichte, Videos,Computerprogramme)

14.10Neue Apps (Programme) auf ein mobiles Gerät herunterladen (z. B. Smartphone)

14.11Für schulische Aufgaben im Internet surfen.

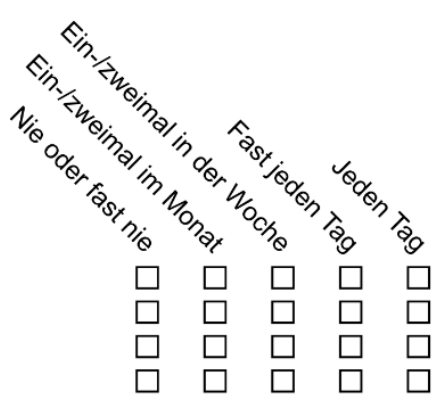

15. Weitere Angaben zur Internetnutzung

15.1 Wie viel Zeit verbringen Sie durchschnittlich am Tag ausschließlich für Fernsehen und das Ansehen von Videos oder Videoclips (auf Fernsehgerät, PC, Smartphone, Tablet, etc.)?
$\square$ weniger als $1 / 2$ Stunde
$\square 1 / 2$ - 1 Stunde
$\square$ 1-2 Stunden
$\square$ 2-3 Stunden
$\square$ 3-4 Stunden
$\square$ mehr als 4 Stunden

15.2 Lassen Sie nun Fernseh- und Videozeit außer Acht: Wie viel Zeit verbringen Sie durchschnittlich am Tag für sämtliche Tätigkeiten, die das Internet voraussetzen (auf Smartphone, PC oder Tablet für soziale Netzwerke, Spiele, Surfen, Informationen suchen,...)?
$\square$ weniger als $1 / 2$ Stunde
$\square 1 / 2$ - 1 Stunde
$\square$ 1-2 Stunden
$\square$ 2-3 Stunden
$\square$ 3-4 Stunden
$\square$ mehr als 4 Stunden

15.3 Sehen Sie sich Erklär- und Lernvideos (z.B. auf YouTube) an, wenn Sie in der Schule etwas nicht verstanden haben oder sich auf einen Test vorbereiten?
$\square$ Nie oder fast nie
$\square$ Hin und wieder
Regelmäßig
$\square$ Häufig

15.4 Hatten Sie in einem Fach schon einmal Hausaufgaben auf, bei denen Sie sich Erklär- und Lernvideos anschauen sollten?
$\square$ Nein, nie
$\square \mathrm{Ja}$, in einem Fach
$\square \mathrm{Ja}$, in mehreren Fächern 
Anhang 4. Fragebogen Pretest Seite 7

\begin{tabular}{l|ll|l} 
EvaSys & 1. Teil - Fragebogen & t03pre2017 Electric Paper
\end{tabular}

16. Wenn Sie über Ihre Erfahrungen mit digitalen Medien und digitalen Geräten nachdenken: Wie sehr stimmen Sie den folgenden Aussagen zu?

16.1 Ich vergesse schnell die Zeit, wenn ich digitale Geräte nutze.

16.2 Das Internet ist eine tolle Quelle, um an interessante Informationen zu gelangen (z. B. Nachrichten, Sport, Wörterbuch).

16.3 Es ist sehr nützlich, soziale Netzwerke im Internet zu haben.

16.4 Ich bin sehr begeistert, wenn ich neue digitale Geräte oder Anwendungen erkunde.

16.5 Ich fühle mich wirklich schlecht, wenn gerade keine Verbindung zum Internet möglich ist.

16.6 Ich mag es, digitale Geräte zu nutzen.

17. Wie sehr stimmen Sie den folgenden Aussagen zu?

17.1 Ich fühle mich auch bei der Nutzung digitaler Geräte gut,

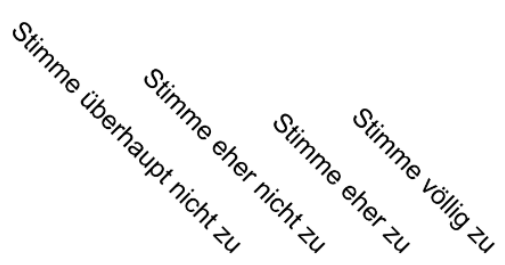

Wenn Freundinnen, Freunde oder Verwandte neue digitale Geräte oder Anwendungen kaufen wollen, kann ich innen Ratschläge geben.

17.3 Ich fühle mich gut, wenn ich zu Hause meine digitalen Geräte nutze.

17.4 Wenn sich ein Problem mit einem digitalen Gerät ergibt, denke ich, dass ich es lösen kann.

17.5 Wenn meine Freundinnen, Freunde oder Verwandte ein Problem mit einem digitalen Gerät haben, kann ich innen helfen.

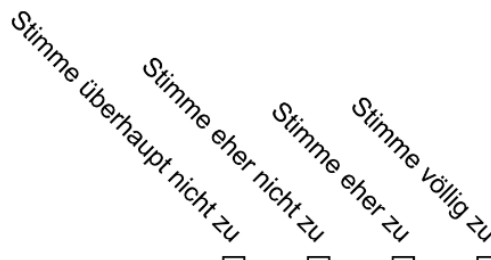

$\square \quad \square \quad \square \quad \square$

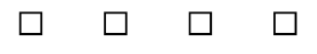




\section{Anhang 5. Fragebogen Posttest Kontrollgruppe Seite 1}

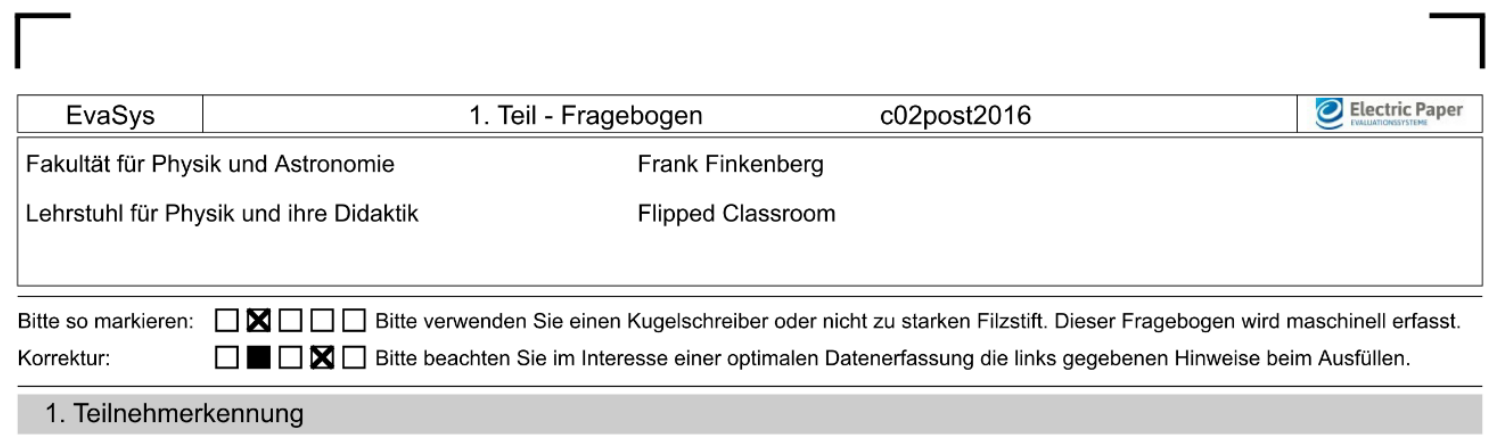

\subsection{Nennen Sie hier Ihre TN-ID:}

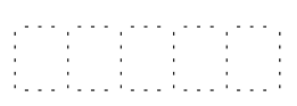

\section{Allgemeine Angaben}
2.1 Sind Sie weiblich oder männlich?
$\square$ weiblich
$\square$ männlich
2.2 Welche Schule besuchen Sie?
$\square$ Deutschhaus-
$\square$ Wirsberggymnasium

3. Wie sehr stimmen Sie folgenden Aussagen in Bezug auf Ihre Person zu?

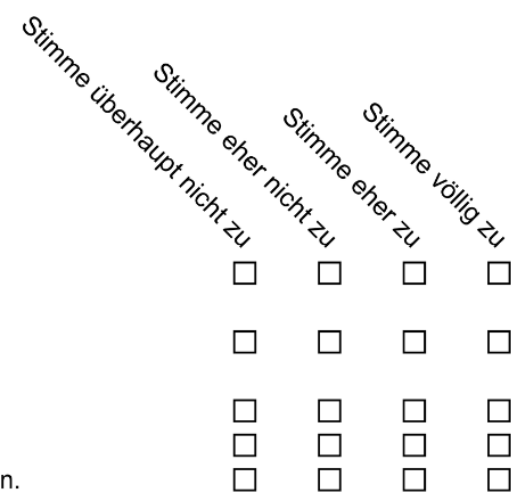

3.1 Ich glaube, dass ich anspruchsvollen Stoff im Physikunterricht leicht lernen kann.

3.2 Normalerweise kann ich Fragen im Physikunterricht gut beantworten.

3.3 Ich lerne neuen Stoff im Physikunterricht schnell.

3.4 Den Stoff im Physikunterricht finde ich einfach.

3.5 Es fällt mir leicht, neue Ideen im Physikunterricht zu verstehen.

4. Wie sehr stimmen Sie folgenden Aussagen in Bezug auf Ihre Person zu?

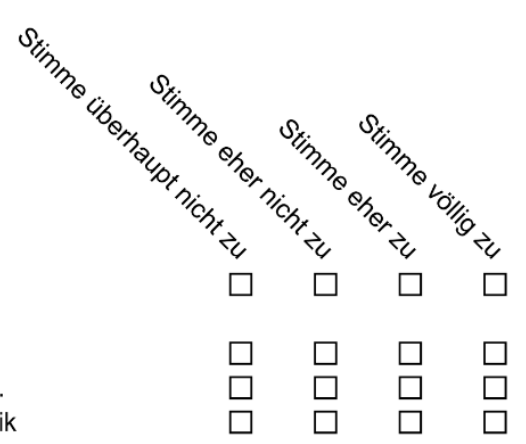

4.1 Im Allgemeinen macht es mir Spaß, mich mit physikalischen Themen zu befassen.

4.2 Ich lese gerne etwas über Physik.

4.3 Ich beschäftige mich gerne mit physikalischen Problemen.

4.4 Ich beschäftige mich in meiner Freizeit mit Themen der Physik 
Anhang 5. Fragebogen Posttest Kontrollgruppe Seite 2

\begin{tabular}{l|ll|l} 
EvaSys & 1. Teil - Fragebogen & c02post2016 & Electric Paper
\end{tabular}

5. Denken Sie an die letzten zwei Monate: Wie sehr waren Sie an den folgenden Schulfächern interessiert?

5.1 Deutsch

5.2 Mathematik

5.3 Chemie

5.4 Physik

5.5 Biologie
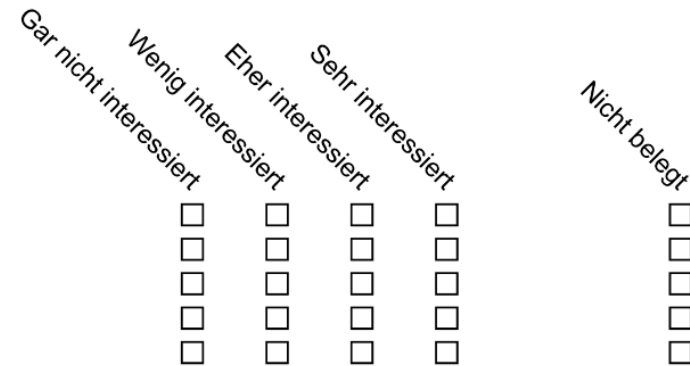

6. Denken Sie an die letzten zwei Monate: Wie oft kam Folgendes in Ihrem Physikunterricht vor?

6.1 Unser Physiklehrer interessierte sich für den Lernfortschritt

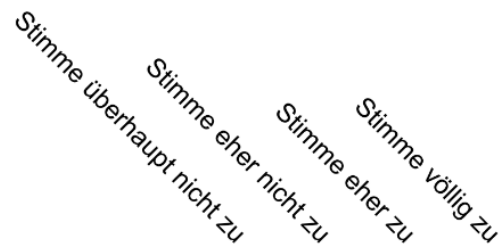
jedes einzelnen Schülers.

6.2 Unser Physiklehrer unterstützte uns zusätzlich, wenn wir Hilfe brauchten.

6.3 Unser Physiklehrer unterstützte uns beim Lernen.

6.4 Unser Physiklehrer erklärte uns etwas so lange, bis wir es verstanden haben.

6.5 Unser Physiklehrer gab uns Gelegenheit, unsere Meinung zu sagen.

7. Interesse an der Unterrichtsreihe

7.1 Die Inhalte des Themas ,Induktion und Schwingkreis' fand

ich interessanter als bisherige Themen in der 11. Jgst.

(elektrische Felder, magnetische Felder, Bewegung von

Teilchen in Feldern, Relativitätstheorie)

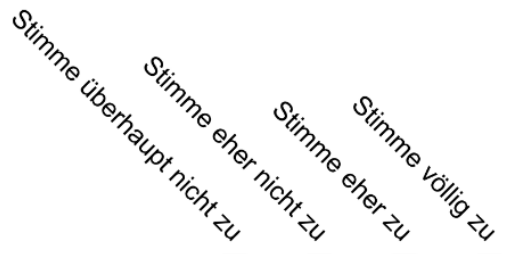


Anhang 5. Fragebogen Posttest Kontrollgruppe Seite 3

\begin{tabular}{|l|lll} 
EvaSys & 1. Teil - Fragebogen & c02post2016 Electric Paper
\end{tabular}

8. Wie sehr stimmen Sie folgenden Aussagen in Bezug auf Ihre Person zu?

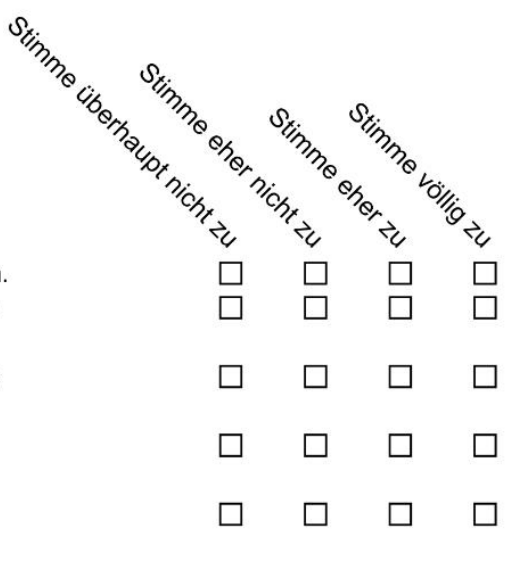

\section{Weitere Angaben}

9.1 Seien Sie ehrlich: Wie regelmäßig haben Sie in den letzten 2 Monaten die Physikhausaufgaben erledigt?
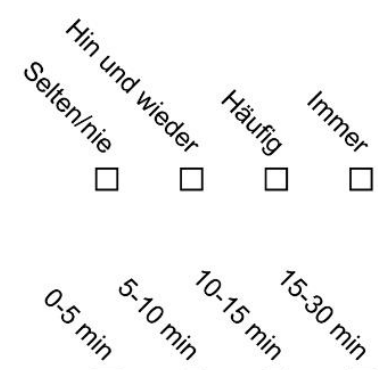

9.2 Wie viel Zeit haben Sie während der letzten 2

Monate durchschnittlich für eine Physikhausaufgabe investiert?

9.3 Wie viele Punkte hatten Sie im Fach Physik im Halbjahreszeugnis der 11. Jahrgangsstufe?
$\square 0$
$\square 1$
$\square 2$
$\square 3$
$\square 4$
$\square 5$
$\square 6$
$\square 7$
$\square 8$
$\square 9$
$\square 10$
$\square 11$
$\square 12$
$\square 13$
$\square 14$
$\square 15$
$\square$ weiß nicht mehr

9.4 Wie viele Punkte würden Sie sich im Fach Physik für den Zeitraum der letzten 2 Monate selbst geben?

$\begin{array}{lll}\square 0 & \square 1 & \square 2 \\ \square 3 & \square 4 & \square 5 \\ \square 6 & \square 7 & \square 8 \\ \square 9 & \square 10 & \square 11 \\ \square 12 & \square 13 & \square 14 \\ \square 15 & & \end{array}$

9.5 Möchten Sie das Halbjahr Physik 11/2 ins

$\square$ Nein

$\square$ Vielleicht

$\square \mathrm{Ja}$ Abitur einbringen? 
Anhang 6. Fragebogen Posttest Treatmentgruppe Seite 1

\begin{tabular}{|c|cc|c|}
\hline EvaSys & 1. Teil - Fragebogen & t04post2017 & Electric Paper \\
\hline \hline Fakultät für Physik und Astronomie & Frank Finkenberg & \\
Lehrstuhl für Physik und ihre Didaktik & Flipped Classroom & \\
& & \\
& &
\end{tabular}

Bitte so markieren: $\square \bigotimes \square \square \square$ Bitte verwenden Sie einen Kugelschreiber oder nicht zu starken Filzstift. Dieser Fragebogen wird maschinell erfasst. Korrektur: $\quad \square \square \square \bigotimes \square$ Bitte beachten Sie im Interesse einer optimalen Datenerfassung die links gegebenen Hinweise beim Ausfüllen.

1. Teilnehmerkennung

1.1 Nennen Sie hier Ihre TN-ID:

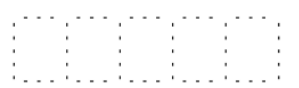

\section{Allgemeine Angaben}

2.1 Sind Sie weiblich oder männlich?

$\square$ weiblich $\square$ männlich

2.2 Welche Schule besuchen Sie?

3. Wie sehr stimmen Sie folgenden Aussagen in Bezug auf Ihre Person zu?

3.1 Ich glaube, dass ich anspruchsvollen Stoff im Physikunterricht leicht lernen kann.

3.2 Normalerweise kann ich Fragen im Physikunterricht gut beantworten.

3.3 Ich lerne neuen Stoff im Physikunterricht schnell.

3.4 Den Stoff im Physikunterricht finde ich einfach.

3.5 Es fällt mir leicht, neue Ideen im Physikunterricht zu verstehen.

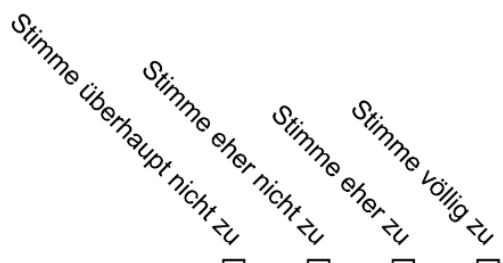

4. Wie sehr stimmen Sie folgenden Aussagen in Bezug auf Ihre Person zu?

4.1 Im Allgemeinen macht es mir Spaß, mich mit physikalischen Themen zu befassen.

4.2 Ich lese gerne etwas über Physik.

4.3 Ich beschäftige mich gerne mit physikalischen Problemen

4.4 Ich beschäftige mich in meiner Freizeit mit Themen der Physik

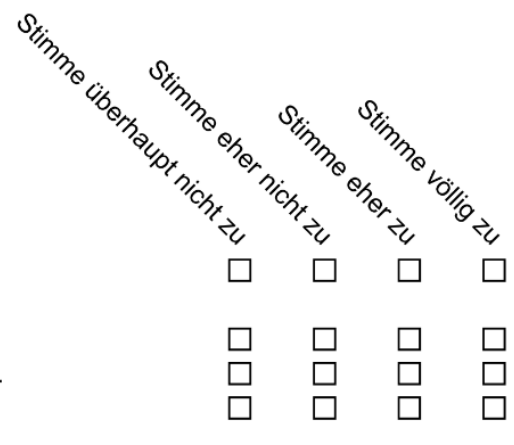

F5594U49562609P1PL1V1 
Anhang 6. Fragebogen Posttest Treatmentgruppe Seite 2

\begin{tabular}{l|l} 
EvaSys & 1. Teil - Fragebogen
\end{tabular}

t04post2017

O Electric Paper

5. Denken Sie an die letzten zwei Monate: Wie sehr waren Sie an den folgenden Schulfächern interessiert?

5.1 Deutsch

5.2 Mathematik

5.3 Chemie

5.4 Physik

5.5 Biologie

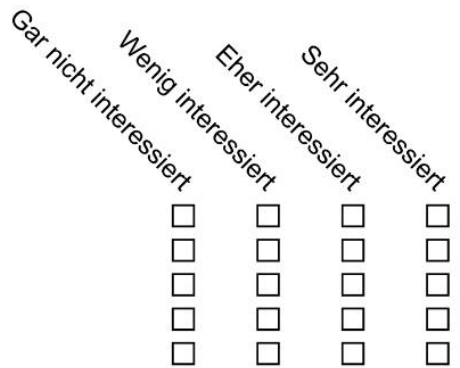

6. Denken Sie an die letzten zwei Monate: Wie oft kam Folgendes in Ihrem Physikunterricht vor?

6.1 Unser Physiklehrer interessierte sich für den Lernfortschritt

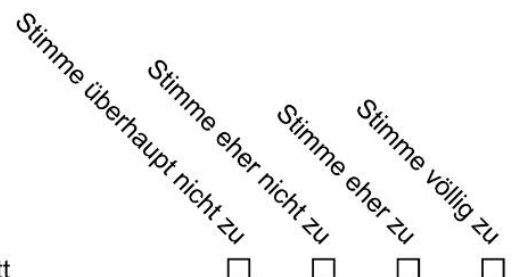
jedes einzelnen Schülers.

6.2 Unser Physiklehrer unterstützte uns zusätzlich, wenn wir Hilfe brauchten.

6.3 Unser Physiklehrer unterstützte uns beim Lernen.

6.4 Unser Physiklehrer erklärte uns etwas so lange, bis wir es verstanden haben.

6.5 Unser Physiklehrer gab uns Gelegenheit, unsere Meinung zu sagen.

7. Interesse an der Unterrichtsreihe

7.1 Die Inhalte des Themas ,Induktion und Schwingkreis' fand

ich interessanter als bisherige Themen in der 11. Jgst.

(elektrische Felder, magnetische Felder, Bewegung von

Teilchen in Feldern)

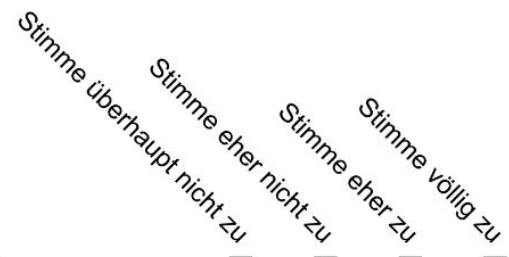

re 
Anhang 6. Fragebogen Posttest Treatmentgruppe Seite 3

\begin{tabular}{l|ll|l} 
EvaSys & 1. Teil - Fragebogen & t04post2017 Electric Paper
\end{tabular}

8. Wie sehr stimmen Sie folgenden Aussagen in Bezug auf Ihre Person zu?

8.1 Ich habe das Thema, Induktion und Schwingkreis' gut

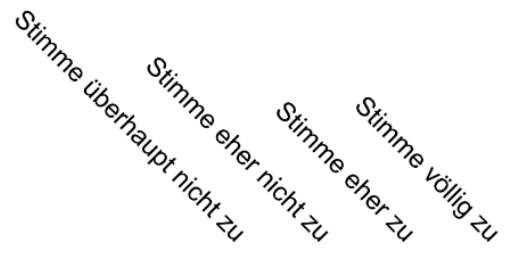
verstanden.

8.2 Ich kann auch schwierige Aufgaben zum Thema ,Induktion und Schwingkreis' lösen.

8.3 Unterrichtsfragen zum Thema, Induktion und Schwingkreis 'kann ich leicht beantworten.

8.4 Ich kann anderen Schülern Sachverhalte zum Thema ,Induktion und Schwingkreis' gut erklären.

8.5 Ich kann clevere Fragen zum Thema ,Induktion und Schwingkreis' stellen.

\section{Weitere Angaben}

9.1 Seien Sie ehrlich: Wie regelmäßig haben Sie in den letzten 2 Monaten die Physikhausaufgaben erledigt?
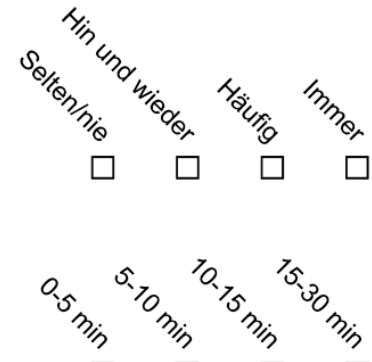

9.2 Wie viel Zeit haben Sie während der letzten 2 Monate durchschnittlich für eine Physikhausaufgabe investiert?

9.3 Wie viele Punkte hatten Sie im Fach Physik im Halbjahreszeugnis der 11. Jahrgangsstufe?
$\square 0$
$\square 1$
$\square 2$
$\square 3$
$\square 4$
$\square 7$
$\square 5$
$\square 6$
$\square 10$
$\square 8$
$\square 9$
$\square 13$
$\square 11$
$\square 12$
$\square$ weiß nicht mehr
$\square 14$

9.4 Wie viele Punkte würden Sie sich im Fach Physik für den Zeitraum der letzten 2 Monate selbst geben?
$\square 0$
$\square 1$
$\square 2$
$\square 3$
$\square 4$
$\square 7$
$\square 10$
$\square 5$
$\square 6$
$\square 13$
$\square 8$
$\square 12$
$\square 15$
$\square 11$
$\square 14$

9.5 Möchten Sie das Halbjahr Physik 11/2 ins

$\square$ Nein

Vielleicht

$\square \mathrm{Ja}$ Abitur einbringen? 
Anhang 6. Fragebogen Posttest Treatmentgruppe Seite 4

\begin{tabular}{l|l} 
EvaSys & 1. Teil - Fragebogen
\end{tabular}

t04post2017

을 Electric Paper

10. Wie sehr stimmen Sie folgenden Aussagen in Bezug auf Ihre Person zu?

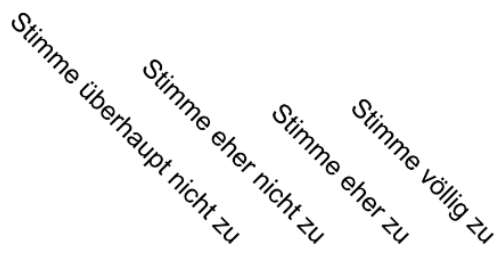

10.1 Mit der Flipped Classroom Methode habe ich mehr gelernt im Vergleich zum normalen Unterricht.

10.2 Bei dieser Methode musste ich zuhause mehr tun als im normalen Unterricht.

10.3 Bei dieser Methode musste ich im Unterricht mehr tun als im normalen Unterricht.

10.4 Bei dieser Methode habe ich aktiver mit meinen Mitschülern gearbeitet als im normalen Unterricht.

10.5 Mein Physiklehrer konnte bei Problemen, die ich hatte, besser auf mich eingehen.

10.6 Ich glaube, mein Physiklehrer hat bei dieser Methode ein besseres Bild davon, was ich kann und was nicht.

11. Wie sehr stimmen Sie folgenden Aussagen in Bezug auf Ihre Person zu?

11.1 Wenn ich die Experimente live statt nur im Video gesehen

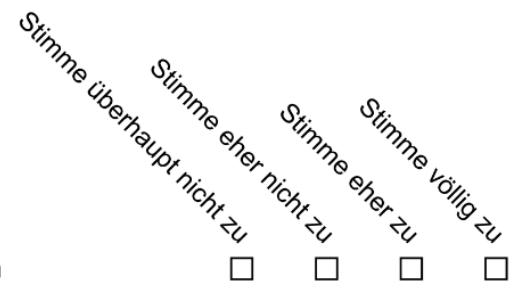
hätte, hätte ich mehr davon gehabt.

11.2 Das typische Lehrer-Schüler-Gespräch, mit dem

gemeinsam die Inhalte entwickelt werden, hat mir bei der Flipped Classroom Methode gefehlt.

11.3 Ich war mit der Vermittlung der Inhalte durch die Videos überfordert.

11.4 Diese Methode fand ich für acht Wochen zu lang und zu eintönig.

11.5 Ich hatte technische Schwierigkeiten, die Videos anzuschauen.

12. Wie sehr hat der Einsatz der Lernvideos die Zeit verändert, die Sie insgesamt vor einem Bildschirm verbracht haben?

12.1 Wie sehr hat der Einsatz der Videos die Zeit verändert, die Sie insgesamt vor einem Bildschirm verbracht haben? $\square$ Die Gesamtzeit wurde nicht $\quad \square$ Die Gesamtzeit wurde ein wenig $\square$ Die Gesamtzeit wurde deutlich wirklich länger.

$$
\text { länger. }
$$

$$
\text { länger. }
$$


Anhang 7. Vorlage offenes Feedback für die Treatmentgruppe

TN-ID:

Feedback zur Flipped Classroom Methode

1. Was fanden Sie positiv an der Flipped Classroom Methode? (Stichpunkte)

2. Was fanden Sie negativ an der Flipped Classroom Methode? (Stichpunkte) 
Bisher erschienene Bände der Reihe „Studien zum Physik- und Chemielernen“

ISSN 1614-8967（vormals Studien zum Physiklernen ISSN 1435-5280)

1 Helmut Fischler, Jochen Peuckert (Hrsg.): Concept Mapping in fachdidaktischen Forschungsprojekten der Physik und Chemie

ISBN 978-3-89722-256-4 40.50 EUR

2 Anja Schoster: Bedeutungsentwicklungsprozesse beim Lösen algorithmischer Physikaufgaben. Eine Fallstudie zu Lernprozessen von Schülern im Physiknachhilfeunterricht während der Bearbeitung algorithmischer Physikaufgaben

ISBN 978-3-89722-045-4 40.50 EUR

3 Claudia von Aufschnaiter: Bedeutungsentwicklungen, Interaktionen und situatives Erleben beim Bearbeiten physikalischer Aufgaben

ISBN 978-3-89722-143-7 40.50 EUR

4 Susanne Haeberlen: Lernprozesse im Unterricht mit Wasserstromkreisen. Eine Fallstudie in der Sekundarstufe I

ISBN 978-3-89722-172-7 40.50 EUR

5 Kerstin Haller: Über den Zusammenhang von Handlungen und Zielen. Eine empirische Untersuchung zu Lernprozessen im physikalischen Praktikum

ISBN 978-3-89722-242-7 40.50 EUR

6 Michaela Horstendahl: Motivationale Orientierungen im Physikunterricht ISBN 978-3-89722-227-4 50.00 EUR

7 Stefan Deylitz: Lernergebnisse in der Quanten-Atomphysik. Evaluation des Bremer Unterrichtskonzepts

ISBN 978-3-89722-291-5 40.50 EUR

8 Lorenz Hucke: Handlungsregulation und Wissenserwerb in traditionellen und computergestützten Experimenten des physikalischen Praktikums

ISBN 978-3-89722-316-5 50.00 EUR

9 Heike Theyßen: Ein Physikpraktikum für Studierende der Medizin. Darstellung der Entwicklung und Evaluation eines adressatenspezifischen Praktikums nach dem Modell der Didaktischen Rekonstruktion

ISBN 978-3-89722-334-9 40.50 EUR

10 Annette Schick: Der Einfluß von Interesse und anderen selbstbezogenen Kognitionen auf Handlungen im Physikunterricht. Fallstudien zu Interessenhandlungen im Physikunterricht

ISBN 978-3-89722-380-6 40.50 EUR

11 Roland Berger: Moderne bildgebende Verfahren der medizinischen Diagnostik. Ein Weg zu interessanterem Physikunterricht

ISBN 978-3-89722-445-2 40.50 EUR 
12 Johannes Werner: Vom Licht zum Atom. Ein Unterrichtskonzept zur Quantenphysik unter Nutzung des Zeigermodells

ISBN 978-3-89722-471-1 40.50 EUR

13 Florian Sander: Verbindung von Theorie und Experiment im physikalischen Praktikum. Eine empirische Untersuchung zum handlungsbezogenen Vorverständnis und dem Einsatz grafikorientierter Modellbildung im Praktikum

ISBN 978-3-89722-482-7 40.50 EUR

14 Jörn Gerdes: Der Begriff der physikalischen Kompetenz. Zur Validierung eines Konstruktes

ISBN 978-3-89722-510-7 40.50 EUR

15 Malte Meyer-Arndt: Interaktionen im Physikpraktikum zwischen Studierenden und Betreuern. Feldstudie zu Bedeutungsentwicklungsprozessen im physikalischen Praktikum

ISBN 978-3-89722-541-1 40.50 EUR

16 Dietmar Höttecke: Die Natur der Naturwissenschaften historisch verstehen. Fachdidaktische und wissenschaftshistorische Untersuchungen

ISBN 978-3-89722-607-4 40.50 EUR

17 Gil Gabriel Mavanga: Entwicklung und Evaluation eines experimentell- und phänomenorientierten Optikcurriculums. Untersuchung zu Schülervorstellungen in der Sekundarstufe I in Mosambik und Deutschland

ISBN 978-3-89722-721-7 40.50 EUR

18 Meike Ute Zastrow: Interaktive Experimentieranleitungen. Entwicklung und Evaluation eines Konzeptes zur Vorbereitung auf das Experimentieren mit Messgeräten im Physikalischen Praktikum

ISBN 978-3-89722-802-3 40.50 EUR

19 Gunnar Friege: Wissen und Problemlösen. Eine empirische Untersuchung des wissenszentrierten Problemlösens im Gebiet der Elektrizitätslehre auf der Grundlage des Experten-Novizen-Vergleichs

ISBN 978-3-89722-809-2 40.50 EUR

20 Erich Starauschek: Physikunterricht nach dem Karlsruher Physikkurs. Ergebnisse einer Evaluationsstudie

ISBN 978-3-89722-823-8 40.50 EUR

21 Roland Paatz: Charakteristika analogiebasierten Denkens. Vergleich von Lernprozessen in Basis- und Zielbereich

ISBN 978-3-89722-944-0 40.50 EUR

22 Silke Mikelskis-Seifert: Die Entwicklung von Metakonzepten zur Teilchenvorstellung bei Schülern. Untersuchung eines Unterrichts über Modelle mithilfe eines Systems multipler Repräsentationsebenen

ISBN 978-3-8325-0013-9 40.50 EUR

23 Brunhild Landwehr: Distanzen von Lehrkräften und Studierenden des Sachunterrichts zur Physik. Eine qualitativ-empirische Studie zu den Ursachen

ISBN 978-3-8325-0044-3 40.50 EUR 
24 Lydia Murmann: Physiklernen zu Licht, Schatten und Sehen. Eine phänomenografische Untersuchung in der Primarstufe

ISBN 978-3-8325-0060-3 40.50 EUR

25 Thorsten Bell: Strukturprinzipien der Selbstregulation. Komplexe Systeme, Elementarisierungen und Lernprozessstudien für den Unterricht der Sekundarstufe II

ISBN 978-3-8325-0134-1 40.50 EUR

26 Rainer Müller: Quantenphysik in der Schule

ISBN 978-3-8325-0186-0 40.50 EUR

27 Jutta Roth: Bedeutungsentwicklungsprozesse von Physikerinnen und Physikern in den Dimensionen Komplexität, Zeit und Inhalt

ISBN 978-3-8325-0183-9 40.50 EUR

28 Andreas Saniter: Spezifika der Verhaltensmuster fortgeschrittener Studierender der Physik

ISBN 978-3-8325-0292-8 40.50 EUR

29 Thomas Weber: Kumulatives Lernen im Physikunterricht. Eine vergleichende Untersuchung in Unterrichtsgängen zur geometrischen Optik

ISBN 978-3-8325-0316-1 40.50 EUR

30 Markus Rehm: Über die Chancen und Grenzen moralischer Erziehung im naturwissenschaftlichen Unterricht

ISBN 978-3-8325-0368-0 40.50 EUR

31 Marion Budde: Lernwirkungen in der Quanten-Atom-Physik. Fallstudien über Resonanzen zwischen Lernangeboten und SchülerInnen-Vorstellungen

ISBN 978-3-8325-0483-0 40.50 EUR

32 Thomas Reyer: Oberflächenmerkmale und Tiefenstrukturen im Unterricht. Exemplarische Analysen im Physikunterricht der gymnasialen Sekundarstufe

ISBN 978-3-8325-0488-5 40.50 EUR

33 Christoph Thomas Müller: Subjektive Theorien und handlungsleitende Kognitionen von Lehrern als Determinanten schulischer Lehr-Lern-Prozesse im Physikunterricht ISBN 978-3-8325-0543-1 40.50 EUR

34 Gabriela Jonas-Ahrend: Physiklehrervorstellungen zum Experiment im Physikunterricht

ISBN 978-3-8325-0576-9 40.50 EUR

35 Dimitrios Stavrou: Das Zusammenspiel von Zufall und Gesetzmäßigkeiten in der nichtlinearen Dynamik. Didaktische Analyse und Lernprozesse

ISBN 978-3-8325-0609-4 40.50 EUR

36 Katrin Engeln: Schülerlabors: authentische, aktivierende Lernumgebungen als Möglichkeit, Interesse an Naturwissenschaften und Technik zu wecken ISBN 978-3-8325-0689-6 40.50 EUR

37 Susann Hartmann: Erklärungsvielfalt

ISBN 978-3-8325-0730-5 40.50 EUR 
Knut Neumann: Didaktische Rekonstruktion eines physikalischen Praktikums für Physiker

ISBN 978-3-8325-0762-6 40.50 EUR

39 Michael Späth: Kontextbedingungen für Physikunterricht an der Hauptschule. Möglichkeiten und Ansatzpunkte für einen fachübergreifenden, handlungsorientierten und berufsorientierten Unterricht

ISBN 978-3-8325-0827-2 40.50 EUR

40 Jörg Hirsch: Interesse, Handlungen und situatives Erleben von Schülerinnen und Schülern beim Bearbeiten physikalischer Aufgaben

ISBN 978-3-8325-0875-3 40.50 EUR

41 Monika Hüther: Evaluation einer hypermedialen Lernumgebung zum Thema Gasgesetze. Eine Studie im Rahmen des Physikpraktikums für Studierende der Medizin ISBN 978-3-8325-0911-8 40.50 EUR

42 Maike Tesch: Das Experiment im Physikunterricht. Didaktische Konzepte und Ergebnisse einer Videostudie

ISBN 978-3-8325-0975-0 40.50 EUR

43 Nina Nicolai: Skriptgeleitete Eltern-Kind-Interaktion bei Chemiehausaufgaben. Eine Evaluationsstudie im Themenbereich Säure-Base

ISBN 978-3-8325-1013-8 40.50 EUR

44 Antje Leisner: Entwicklung von Modellkompetenz im Physikunterricht ISBN 978-3-8325-1020-6 40.50 EUR

45 Stefan Rumann: Evaluation einer Interventionsstudie zur Säure-Base-Thematik ISBN 978-3-8325-1027-5 40.50 EUR

46 Thomas Wilhelm: Konzeption und Evaluation eines Kinematik/Dynamik-Lehrgangs zur Veränderung von Schülervorstellungen mit Hilfe dynamisch ikonischer Repräsentationen und graphischer Modellbildung - mit CD-ROM

ISBN 978-3-8325-1046-6 45.50 EUR

47 Andrea Maier-Richter: Computerunterstütztes Lernen mit Lösungsbeispielen in der Chemie. Eine Evaluationsstudie im Themenbereich Löslichkeit ISBN 978-3-8325-1046-6 40.50 EUR

48 Jochen Peuckert: Stabilität und Ausprägung kognitiver Strukturen zum Atombegriff ISBN 978-3-8325-1104-3 40.50 EUR

49 Maik Walpuski: Optimierung von experimenteller Kleingruppenarbeit durch Strukturierungshilfen und Feedback

ISBN 978-3-8325-1184-5 40.50 EUR

50 Helmut Fischler, Christiane S. Reiners (Hrsg.): Die Teilchenstruktur der Materie im Physik- und Chemieunterricht

ISBN 978-3-8325-1225-5 34.90 EUR

51 Claudia Eysel: Interdisziplinäres Lehren und Lernen in der Lehrerbildung. Eine empirische Studie zum Kompetenzerwerb in einer komplexen Lernumgebung

ISBN 978-3-8325-1238-5 40.50 EUR 
52 Johannes Günther: Lehrerfortbildung über die Natur der Naturwissenschaften.

Studien über das Wissenschaftsverständnis von Grundschullehrkräften

ISBN 978-3-8325-1287-3 40.50 EUR

53 Christoph Neugebauer: Lernen mit Simulationen und der Einfluss auf das Problemlösen in der Physik

ISBN 978-3-8325-1300-9 40.50 EUR

54 Andreas Schnirch: Gendergerechte Interessen- und Motivationsförderung im Kontext naturwissenschaftlicher Grundbildung. Konzeption, Entwicklung und Evaluation einer multimedial unterstützten Lernumgebung

ISBN 978-3-8325-1334-4 40.50 EUR

55 Hilde Köster: Freies Explorieren und Experimentieren. Eine Untersuchung zur selbstbestimmten Gewinnung von Erfahrungen mit physikalischen Phänomenen im Sachunterricht

ISBN 978-3-8325-1348-1 40.50 EUR

56 Eva Heran-Dörr: Entwicklung und Evaluation einer Lehrerfortbildung zur Förderung der physikdidaktischen Kompetenz von Sachunterrichtslehrkräften ISBN 978-3-8325-1377-1 40.50 EUR

57 Agnes Szabone Varnai: Unterstützung des Problemlösens in Physik durch den Einsatz von Simulationen und die Vorgabe eines strukturierten Kooperationsformats ISBN 978-3-8325-1403-7 40.50 EUR

58 Johannes Rethfeld: Aufgabenbasierte Lernprozesse in selbstorganisationsoffenem Unterricht der Sekundarstufe I zum Themengebiet ELEKTROSTATIK. Eine Feldstudie in vier 10. Klassen zu einer kartenbasierten Lernumgebung mit Aufgaben aus der Elektrostatik

ISBN 978-3-8325-1416-7 40.50 EUR

59 Christian Henke: Experimentell-naturwissenschaftliche Arbeitsweisen in der Oberstufe. Untersuchung am Beispiel des HIGHSEA-Projekts in Bremerhaven ISBN 978-3-8325-1515-7 40.50 EUR

60 Lutz Kasper: Diskursiv-narrative Elemente für den Physikunterricht. Entwicklung und Evaluation einer multimedialen Lernumgebung zum Erdmagnetismus ISBN 978-3-8325-1537-9 40.50 EUR

61 Thorid Rabe: Textgestaltung und Aufforderung zu Selbsterklärungen beim Physiklernen mit Multimedia ISBN 978-3-8325-1539-3 40.50 EUR

62 Ina Glemnitz: Vertikale Vernetzung im Chemieunterricht. Ein Vergleich von traditionellem Unterricht mit Unterricht nach Chemie im Kontext

ISBN 978-3-8325-1628-4 40.50 EUR

63 Erik Einhaus: Schülerkompetenzen im Bereich Wärmelehre. Entwicklung eines Testinstruments zur Überprüfung und Weiterentwicklung eines normativen Modells fachbezogener Kompetenzen

ISBN 978-3-8325-1630-7 40.50 EUR 
64 Jasmin Neuroth: Concept Mapping als Lernstrategie. Eine Interventionsstudie zum Chemielernen aus Texten

ISBN 978-3-8325-1659-8 40.50 EUR

65 Hans Gerd Hegeler-Burkhart: Zur Kommunikation von Hauptschülerinnen und Hauptschülern in einem handlungsorientierten und fächerübergreifenden Unterricht mit physikalischen und technischen Inhalten

ISBN 978-3-8325-1667-3 40.50 EUR

66 Karsten Rincke: Sprachentwicklung und Fachlernen im Mechanikunterricht. Sprache und Kommunikation bei der Einführung in den Kraftbegriff

ISBN 978-3-8325-1699-4 40.50 EUR

67 Nina Strehle: Das Ion im Chemieunterricht. Alternative Schülervorstellungen und curriculare Konsequenzen

ISBN 978-3-8325-1710-6 40.50 EUR

68 Martin Hopf: Problemorientierte Schülerexperimente

ISBN 978-3-8325-1711-3 40.50 EUR

69 Anne Beerenwinkel: Fostering conceptual change in chemistry classes using expository texts

ISBN 978-3-8325-1721-2 40.50 EUR

70 Roland Berger: Das Gruppenpuzzle im Physikunterricht der Sekundarstufe II. Eine empirische Untersuchung auf der Grundlage der Selbstbestimmungstheorie der Motivation

ISBN 978-3-8325-1732-8 40.50 EUR

71 Giuseppe Colicchia: Physikunterricht im Kontext von Medizin und Biologie. Entwicklung und Erprobung von Unterrichtseinheiten ISBN 978-3-8325-1746-5 40.50 EUR

72 Sandra Winheller: Geschlechtsspezifische Auswirkungen der Lehrer-Schüler-Interaktion im Chemieanfangsunterricht

ISBN 978-3-8325-1757-1 40.50 EUR

73 Isabel Wahser: Training von naturwissenschaftlichen Arbeitsweisen zur Unterstützung experimenteller Kleingruppenarbeit im Fach Chemie

ISBN 978-3-8325-1815-8 40.50 EUR

74 Claus Brell: Lernmedien und Lernerfolg - reale und virtuelle Materialien im Physikunterricht. Empirische Untersuchungen in achten Klassen an Gymnasien (Laborstudie) zum Computereinsatz mit Simulation und IBE

ISBN 978-3-8325-1829-5 40.50 EUR

75 Rainer Wackermann: Überprüfung der Wirksamkeit eines Basismodell-Trainings für Physiklehrer

ISBN 978-3-8325-1882-0 40.50 EUR

76 Oliver Tepner: Effektivität von Aufgaben im Chemieunterricht der Sekundarstufe I ISBN 978-3-8325-1919-3 40.50 EUR 
77 Claudia Geyer: Museums- und Science-Center-Besuche im naturwissenschaftlichen Unterricht aus einer motivationalen Perspektive. Die Sicht von Lehrkräften und Schülerinnen und Schülern

ISBN 978-3-8325-1922-3 40.50 EUR

78 Tobias Leonhard: Professionalisierung in der Lehrerbildung. Eine explorative Studie zur Entwicklung professioneller Kompetenzen in der Lehrererstausbildung ISBN 978-3-8325-1924-7 40.50 EUR

79 Alexander Kauertz: Schwierigkeitserzeugende Merkmale physikalischer Leistungstestaufgaben

ISBN 978-3-8325-1925-4 40.50 EUR

80 Regina Hübinger: Schüler auf Weltreise. Entwicklung und Evaluation von Lehr-/ Lernmaterialien zur Förderung experimentell-naturwissenschaftlicher Kompetenzen für die Jahrgangsstufen 5 und 6

ISBN 978-3-8325-1932-2 40.50 EUR

81 Christine Waltner: Physik lernen im Deutschen Museum

ISBN 978-3-8325-1933-9 40.50 EUR

82 Torsten Fischer: Handlungsmuster von Physiklehrkräften beim Einsatz neuer Medien. Fallstudien zur Unterrichtspraxis

ISBN 978-3-8325-1948-3 42.00 EUR

83 Corinna Kieren: Chemiehausaufgaben in der Sekundarstufe I des Gymnasiums. Fragebogenerhebung zur gegenwärtigen Praxis und Entwicklung eines optimierten Hausaufgabendesigns im Themenbereich Säure-Base

978-3-8325-1975-9 37.00 EUR

84 Marco Thiele: Modelle der Thermohalinen Zirkulation im Unterricht. Eine empirische Studie zur Förderung des Modellverständnisses

ISBN 978-3-8325-1982-7 40.50 EUR

85 Bernd Zinn: Physik lernen, um Physik zu lehren. Eine Möglichkeit für interessanteren Physikunterricht

ISBN 978-3-8325-1995-7 39.50 EUR

86 Esther Klaes: Außerschulische Lernorte im naturwissenschaftlichen Unterricht. Die Perspektive der Lehrkraft

ISBN 978-3-8325-2006-9 43.00 EUR

87 Marita Schmidt: Kompetenzmodellierung und -diagnostik im Themengebiet Energie der Sekundarstufe I. Entwicklung und Erprobung eines Testinventars ISBN 978-3-8325-2024-3 37.00 EUR

88 Gudrun Franke-Braun: Aufgaben mit gestuften Lernhilfen. Ein Aufgabenformat zur Förderung der sachbezogenen Kommunikation und Lernleistung für den naturwissenschaftlichen Unterricht

ISBN 978-3-8325-2026-7 38.00 EUR

89 Silke Klos: Kompetenzförderung im naturwissenschaftlichen Anfangsunterricht. Der Einfluss eines integrierten Unterrichtskonzepts

ISBN 978-3-8325-2133-2 37.00 EUR 
90 Ulrike Elisabeth Burkard: Quantenphysik in der Schule. Bestandsaufnahme, Perspektiven und Weiterentwicklungsmöglichkeiten durch die Implementation eines Medienservers

ISBN 978-3-8325-2215-5 43.00 EUR

91 Ulrike Gromadecki: Argumente in physikalischen Kontexten. Welche Geltungsgründe halten Physikanfänger für überzeugend?

ISBN 978-3-8325-2250-6 41.50 EUR

92 Jürgen Bruns: Auf dem Weg zur Förderung naturwissenschaftsspezifischer Vorstellungen von zukünftigen Chemie-Lehrenden ISBN 978-3-8325-2257-5 43.50 EUR

93 Cornelius Marsch: Räumliche Atomvorstellung. Entwicklung und Erprobung eines Unterrichtskonzeptes mit Hilfe des Computers

ISBN 978-3-8325-2293-3 82.50 EUR

94 Maja Brückmann: Sachstrukturen im Physikunterricht. Ergebnisse einer Videostudie ISBN 978-3-8325-2272-8 39.50 EUR

95 Sabine Fechner: Effects of Context-oriented Learning on Student Interest and Achievement in Chemistry Education

ISBN 978-3-8325-2343-5 36.50 EUR

96 Clemens Nagel: eLearning im Physikalischen Anfängerpraktikum ISBN 978-3-8325-2355-8 39.50 EUR

97 Josef Riese: Professionelles Wissen und professionelle Handlungskompetenz von (angehenden) Physiklehrkräften

ISBN 978-3-8325-2376-3 39.00 EUR

98 Sascha Bernholt: Kompetenzmodellierung in der Chemie. Theoretische und empirische Reflexion am Beispiel des Modells hierarchischer Komplexität ISBN 978-3-8325-2447-0 40.00 EUR

99 Holger Christoph Stawitz: Auswirkung unterschiedlicher Aufgabenprofile auf die Schülerleistung. Vergleich von Naturwissenschafts- und Problemlöseaufgaben der PISA 2003-Studie

ISBN 978-3-8325-2451-7 37.50 EUR

100 Hans Ernst Fischer, Elke Sumfleth (Hrsg.): nwu-essen - 10 Jahre Essener Forschung zum naturwissenschaftlichen Unterricht ISBN 978-3-8325-3331-1 40.00 EUR

101 Hendrik Härtig: Sachstrukturen von Physikschulbüchern als Grundlage zur Bestimmung der Inhaltsvalidität eines Tests

ISBN 978-3-8325-2512-5 34.00 EUR

102 Thomas Grüß-Niehaus: Zum Verständnis des Löslichkeitskonzeptes im Chemieunterricht. Der Effekt von Methoden progressiver und kollaborativer Reflexion ISBN 978-3-8325-2537-8 40.50 EUR

103 Patrick Bronner: Quantenoptische Experimente als Grundlage eines Curriculums zur Quantenphysik des Photons 
ISBN 978-3-8325-2540-8 36.00 EUR

104 Adrian Voßkühler: Blickbewegungsmessung an Versuchsaufbauten. Studien zur Wahrnehmung, Verarbeitung und Usability von physikbezogenen Experimenten am Bildschirm und in der Realität

ISBN 978-3-8325-2548-4 47.50 EUR

105 Verena Tobias: Newton'sche Mechanik im Anfangsunterricht. Die Wirksamkeit einer Einführung über die zweidimensionale Dynamik auf das Lehren und Lernen ISBN 978-3-8325-2558-3 54.00 EUR

106 Christian Rogge: Entwicklung physikalischer Konzepte in aufgabenbasierten Lernumgebungen

ISBN 978-3-8325-2574-3 45.00 EUR

107 Mathias Ropohl: Modellierung von Schülerkompetenzen im Basiskonzept Chemische Reaktion. Entwicklung und Analyse von Testaufgaben

ISBN 978-3-8325-2609-2 36.50 EUR

108 Christoph Kulgemeyer: Physikalische Kommunikationskompetenz. Modellierung und Diagnostik

ISBN 978-3-8325-2674-0 44.50 EUR

109 Jennifer Olszewski: The Impact of Physics Teachers' Pedagogical Content Knowledge on Teacher Actions and Student Outcomes

ISBN 978-3-8325-2680-1 33.50 EUR

110 Annika Ohle: Primary School Teachers' Content Knowledge in Physics and its Impact on Teaching and Students' Achievement

ISBN 978-3-8325-2684-9 36.50 EUR

111 Susanne Mannel: Assessing scientific inquiry. Development and evaluation of a test for the low-performing stage

ISBN 978-3-8325-2761-7 40.00 EUR

112 Michael Plomer: Physik physiologisch passend praktiziert. Eine Studie zur Lernwirksamkeit von traditionellen und adressatenspezifischen Physikpraktika für die Physiologie

ISBN 978-3-8325-2804-1 34.50 EUR

113 Alexandra Schulz: Experimentierspezifische Qualitätsmerkmale im Chemieunterricht. Eine Videostudie

ISBN 978-3-8325-2817-1 40.00 EUR

114 Franz Boczianowski: Eine empirische Untersuchung zu Vektoren im Physikunterricht der Mittelstufe

ISBN 978-3-8325-2843-0 39.50 EUR

115 Maria Ploog: Internetbasiertes Lernen durch Textproduktion im Fach Physik ISBN 978-3-8325-2853-9 39.50 EUR 
116 Anja Dhein: Lernen in Explorier- und Experimentiersituationen. Eine explorative Studie zu Bedeutungsentwicklungsprozessen bei Kindern im Alter zwischen 4 und 6 Jahren

ISBN 978-3-8325-2859-1

45.50 EUR

117 Irene Neumann: Beyond Physics Content Knowledge. Modeling Competence Regarding Nature of Scientific Inquiry and Nature of Scientific Knowledge

ISBN 978-3-8325-2880-5 37.00 EUR

118 Markus Emden: Prozessorientierte Leistungsmessung des naturwissenschaftlichexperimentellen Arbeitens. Eine vergleichende Studie zu Diagnoseinstrumenten zu Beginn der Sekundarstufe I

ISBN 978-3-8325-2867-6 38.00 EUR

119 Birgit Hofmann: Analyse von Blickbewegungen von Schülern beim Lesen von physikbezogenen Texten mit Bildern. Eye Tracking als Methodenwerkzeug in der physikdidaktischen Forschung

ISBN 978-3-8325-2925-3 59.00 EUR

120 Rebecca Knobloch: Analyse der fachinhaltlichen Qualität von Schüleräußerungen und deren Einfluss auf den Lernerfolg. Eine Videostudie zu kooperativer Kleingruppenarbeit ISBN 978-3-8325-3006-8 36.50 EUR

121 Julia Hostenbach: Entwicklung und Prüfung eines Modells zur Beschreibung der Bewertungskompetenz im Chemieunterricht

ISBN 978-3-8325-3013-6 38.00 EUR

122 Anna Windt: Naturwissenschaftliches Experimentieren im Elementarbereich. Evaluation verschiedener Lernsituationen

ISBN 978-3-8325-3020-4 43.50 EUR

123 Eva Kölbach: Kontexteinflüsse beim Lernen mit Lösungsbeispielen ISBN 978-3-8325-3025-9 38.50 EUR

124 Anna Lau: Passung und vertikale Vernetzung im Chemie- und Physikunterricht ISBN 978-3-8325-3021-1 36.00 EUR

125 Jan Lamprecht: Ausbildungswege und Komponenten professioneller Handlungskompetenz. Vergleich von Quereinsteigern mit Lehramtsabsolventen für Gymnasien im Fach Physik

ISBN 978-3-8325-3035-8 38.50 EUR

126 Ulrike Böhm: Förderung von Verstehensprozessen unter Einsatz von Modellen ISBN 978-3-8325-3042-6 41.00 EUR

127 Sabrina Dollny: Entwicklung und Evaluation eines Testinstruments zur Erfassung des fachspezifischen Professionswissens von Chemielehrkräften

ISBN 978-3-8325-3046-4 37.00 EUR 
128 Monika Zimmermann: Naturwissenschaftliche Bildung im Kindergarten. Eine integrative Längsschnittstudie zur Kompetenzentwicklung von Erzieherinnen ISBN 978-3-8325-3053-2 54.00 EUR

129 Ulf Saballus: Über das Schlussfolgern von Schülerinnen und Schülern zu öffentlichen Kontroversen mit naturwissenschaftlichem Hintergrund. Eine Fallstudie ISBN 978-3-8325-3086-0 39.50 EUR

130 Olaf Krey: Zur Rolle der Mathematik in der Physik. Wissenschaftstheoretische Aspekte und Vorstellungen Physiklernender

ISBN 978-3-8325-3101-0 46.00 EUR

131 Angelika Wolf: Zusammenhänge zwischen der Eigenständigkeit im Physikunterricht, der Motivation, den Grundbedürfnissen und dem Lernerfolg von Schülern ISBN 978-3-8325-3161-4 45.00 EUR

132 Johannes Börlin: Das Experiment als Lerngelegenheit. Vom interkulturellen Vergleich des Physikunterrichts zu Merkmalen seiner Qualität

ISBN 978-3-8325-3170-6 45.00 EUR

133 Olaf Uhden: Mathematisches Denken im Physikunterricht. Theorieentwicklung und Problemanalyse

ISBN 978-3-8325-3170-6 45.00 EUR

134 Christoph Gut: Modellierung und Messung experimenteller Kompetenz. Analyse eines large-scale Experimentiertests

ISBN 978-3-8325-3213-0 40.00 EUR

135 Antonio Rueda: Lernen mit ExploMultimedial in kolumbianischen Schulen. Analyse von kurzzeitigen Lernprozessen und der Motivation beim länderübergreifenden Einsatz einer deutschen computergestützten multimedialen Lernumgebung für den naturwissenschaftlichen Unterricht

ISBN 978-3-8325-3218-5 45.50 EUR

136 Krisztina Berger: Bilder, Animationen und Notizen. Empirische Untersuchung zur Wirkung einfacher visueller Repräsentationen und Notizen auf den Wissenserwerb in der Optik

ISBN 978-3-8325-3238-3 41.50 EUR

137 Antony Crossley: Untersuchung des Einflusses unterschiedlicher physikalischer Konzepte auf den Wissenserwerb in der Thermodynamik der Sekundarstufe I ISBN 978-3-8325-3275-8 40.00 EUR

138 Tobias Viering: Entwicklung physikalischer Kompetenz in der Sekundarstufe I. Validierung eines Kompetenzentwicklungsmodells für das Energiekonzept im Bereich Fachwissen

ISBN 978-3-8325-3277-2 37.00 EUR

139 Nico Schreiber: Diagnostik experimenteller Kompetenz. Validierung technologiegestützter Testverfahren im Rahmen eines Kompetenzstrukturmodells ISBN 978-3-8325-3284-0 39.00 EUR 
140 Sarah Hundertmark: Einblicke in kollaborative Lernprozesse. Eine Fallstudie zur reflektierenden Zusammenarbeit unterstützt durch die Methoden Concept Mapping und Lernbegleitbogen

ISBN 978-3-8325-3251-2 43.00 EUR

141 Ronny Scherer: Analyse der Struktur, Messinvarianz und Ausprägung komplexer Problemlösekompetenz im Fach Chemie. Eine Querschnittstudie in der Sekundarstufe I und am Übergang zur Sekundarstufe II ISBN 978-3-8325-3312-0 43.00 EUR

142 Patricia Heitmann: Bewertungskompetenz im Rahmen naturwissenschaftlicher Problemlöseprozesse. Modellierung und Diagnose der Kompetenzen Bewertung und analytisches Problemlösen für das Fach Chemie ISBN 978-3-8325-3314-4 37.00 EUR

143 Jan Fleischhauer: Wissenschaftliches Argumentieren und Entwicklung von Konzepten beim Lernen von Physik ISBN 978-3-8325-3325-0 35.00 EUR

144 Nermin Özcan: Zum Einfluss der Fachsprache auf die Leistung im Fach Chemie. Eine Förderstudie zur Fachsprache im Chemieunterricht ISBN 978-3-8325-3328-1 36.50 EUR

145 Helena van Vorst: Kontextmerkmale und ihr Einfluss auf das Schülerinteresse im Fach Chemie ISBN 978-3-8325-3321-2 38.50 EUR

146 Janine Cappell: Fachspezifische Diagnosekompetenz angehender Physiklehrkräfte in der ersten Ausbildungsphase ISBN 978-3-8325-3356-4 38.50 EUR

147 Susanne Bley: Förderung von Transferprozessen im Chemieunterricht ISBN 978-3-8325-3407-3 40.50 EUR

148 Cathrin Blaes: Die übungsgestützte Lehrerpräsentation im Chemieunterricht der Sekundarstufe I. Evaluation der Effektivität ISBN 978-3-8325-3409-7 43.50 EUR

149 Julia Suckut: Die Wirksamkeit von piko-OWL als Lehrerfortbildung. Eine Evaluation zum Projekt Physik im Kontext in Fallstudien ISBN 978-3-8325-3440-0 45.00 EUR

150 Alexandra Dorschu: Die Wirkung von Kontexten in Physikkompetenztestaufgaben ISBN 978-3-8325-3446-2 37.00 EUR

151 Jochen Scheid: Multiple Repräsentationen, Verständnis physikalischer Experimente und kognitive Aktivierung: Ein Beitrag zur Entwicklung der Aufgabenkultur ISBN 978-3-8325-3449-3 49.00 EUR

152 Tim Plasa: Die Wahrnehmung von Schülerlaboren und Schülerforschungszentren ISBN 978-3-8325-3483-7 35.50 EUR 
153 Felix Schoppmeier: Physikkompetenz in der gymnasialen Oberstufe.Entwicklung und Validierung eines Kompetenzstrukturmodells für den Kompetenzbereich Umgang mit Fachwissen

ISBN 978-3-8325-3502-5 36.00 EUR

154 Katharina Groß: Experimente alternativ dokumentieren. Eine qualitative Studie zur Förderung der Diagnose- und Differenzierungskompetenz in der Chemielehrerbildung ISBN 978-3-8325-3508-7 43.50 EUR

155 Barbara Hank: Konzeptwandelprozesse im Anfangsunterricht Chemie. Eine quasiexperimentelle Längsschnittstudie

ISBN 978-3-8325-3519-3 38.50 EUR

156 Katja Freyer: Zum Einfluss von Studieneingangsvoraussetzungen auf den Studienerfolg Erstsemesterstudierender im Fach Chemie ISBN 978-3-8325-3544-5 38.00 EUR

157 Alexander Rachel: Auswirkungen instruktionaler Hilfen bei der Einführung des (Ferro-)Magnetismus. Eine Vergleichsstudie in der Primar- und Sekundarstufe ISBN 978-3-8325-3548-3 43.50 EUR

158 Sebastian Ritter: Einfluss des Lerninhalts Nanogrößeneffekte auf Teilchen- und Teilchenmodellvorstellungen von Schülerinnen und Schülern

ISBN 978-3-8325-3558-2 36.00 EUR

159 Andrea Harbach: Problemorientierung und Vernetzung in kontextbasierten Lernaufgaben

ISBN 978-3-8325-3564-3 39.00 EUR

160 David Obst: Interaktive Tafeln im Physikunterricht. Entwicklung und Evaluation einer Lehrerfortbildung ISBN 978-3-8325-3582-7 40.50 EUR

161 Sophie Kirschner: Modellierung und Analyse des Professionswissens von Physiklehrkräften

ISBN 978-3-8325-3601-5 35.00 EUR

162 Katja Stief: Selbstregulationsprozesse und Hausaufgabenmotivation im Chemieunterricht

ISBN 978-3-8325-3631-2 34.00 EUR

163 Nicola Meschede: Professionelle Wahrnehmung der inhaltlichen Strukturierung im naturwissenschaftlichen Grundschulunterricht. Theoretische Beschreibung und empirische Erfassung

ISBN 978-3-8325-3668-8 37.00 EUR

164 Johannes Maximilian Barth: Experimentieren im Physikunterricht der gymnasialen Oberstufe. Eine Rekonstruktion übergeordneter Einbettungsstrategien

ISBN 978-3-8325-3681-7 39.00 EUR

165 Sandra Lein: Das Betriebspraktikum in der Lehrerbildung. Eine Untersuchung zur Förderung der Wissenschafts- und Technikbildung im allgemeinbildenden Unterricht ISBN 978-3-8325-3698-5 40.00 EUR 
166 Veranika Maiseyenka: Modellbasiertes Experimentieren im Unterricht. Praxistauglichkeit und Lernwirkungen

ISBN 978-3-8325-3708-1 38.00 EUR

167 Christoph Stolzenberger: Der Einfluss der didaktischen Lernumgebung auf das Erreichen geforderter Bildungsziele am Beispiel der W- und P-Seminare im Fach Physik ISBN 978-3-8325-3708-1 38.00 EUR

168 Pia Altenburger: Mehrebenenregressionsanalysen zum Physiklernen im Sachunterricht der Primarstufe. Ergebnisse einer Evaluationsstudie.

ISBN 978-3-8325-3717-3 37.50 EUR

169 Nora Ferber: Entwicklung und Validierung eines Testinstruments zur Erfassung von Kompetenzentwicklung im Fach Chemie in der Sekundarstufe I

ISBN 978-3-8325-3727-2 39.50 EUR

170 Anita Stender: Unterrichtsplanung: Vom Wissen zum Handeln.

Theoretische Entwicklung und empirische Überprüfung des Transformationsmodells der Unterrichtsplanung

ISBN 978-3-8325-3750-0 41.50 EUR

171 Jenna Koenen: Entwicklung und Evaluation von experimentunterstützten Lösungsbeispielen zur Förderung naturwissenschaftlich-experimenteller Arbeitsweisen ISBN 978-3-8325-3785-2 43.00 EUR

172 Teresa Henning: Empirische Untersuchung kontextorientierter Lernumgebungen in der Hochschuldidaktik. Entwicklung und Evaluation kontextorientierter Aufgaben in der Studieneingangsphase für Fach- und Nebenfachstudierende der Physik ISBN 978-3-8325-3801-9 43.00 EUR

173 Alexander Pusch: Fachspezifische Instrumente zur Diagnose und individuellen Förderung von Lehramtsstudierenden der Physik ISBN 978-3-8325-3829-3 38.00 EUR

174 Christoph Vogelsang: Validierung eines Instruments zur Erfassung der professionellen Handlungskompetenz von (angehenden) Physiklehrkräften. Zusammenhangsanalysen zwischen Lehrerkompetenz und Lehrerperformanz

ISBN 978-3-8325-3846-0 50.50 EUR

175 Ingo Brebeck: Selbstreguliertes Lernen in der Studieneingangsphase im Fach Chemie ISBN 978-3-8325-3859-0 37.00 EUR

176 Axel Eghtessad: Merkmale und Strukturen von Professionalisierungsprozessen in der ersten und zweiten Phase der Chemielehrerbildung. Eine empirisch-qualitative Studie mit niedersächsischen Fachleiter_innen der Sekundarstufenlehrämter ISBN 978-3-8325-3861-3 45.00 EUR

177 Andreas Nehring: Wissenschaftliche Denk- und Arbeitsweisen im Fach Chemie. Eine kompetenzorientierte Modell- und Testentwicklung für den Bereich der Erkenntnisgewinnung

ISBN 978-3-8325-3872-9 39.50 EUR 
178 Maike Schmidt: Professionswissen von Sachunterrichtslehrkräften. Zusammenhangsanalyse zur Wirkung von Ausbildungshintergrund und Unterrichtserfahrung auf das fachspezifische Professionswissen im Unterrichtsinhalt „Verbrennung“ ISBN 978-3-8325-3907-8 38.50 EUR

179 Jan Winkelmann: Auswirkungen auf den Fachwissenszuwachs und auf affektive Schülermerkmale durch Schüler- und Demonstrationsexperimente im Physikunterricht ISBN 978-3-8325-3915-3 41.00 EUR

180 Iwen Kobow: Entwicklung und Validierung eines Testinstrumentes zur Erfassung der Kommunikationskompetenz im Fach Chemie ISBN 978-3-8325-3927-6 34.50 EUR

181 Yvonne Gramzow: Fachdidaktisches Wissen von Lehramtsstudierenden im Fach Physik. Modellierung und Testkonstruktion ISBN 978-3-8325-3931-3 42.50 EUR

182 Evelin Schröter: Entwicklung der Kompetenzerwartung durch Lösen physikalischer Aufgaben einer multimedialen Lernumgebung ISBN 978-3-8325-3975-7 54.50 EUR

183 Inga Kallweit: Effektivität des Einsatzes von Selbsteinschätzungsbögen im Chemieunterricht der Sekundarstufe I. Individuelle Förderung durch selbstreguliertes Lernen ISBN 978-3-8325-3965-8 44.00 EUR

184 Andrea Schumacher: Paving the way towards authentic chemistry teaching. A contribution to teachers' professional development ISBN 978-3-8325-3976-4 48.50 EUR

185 David Woitkowski: Fachliches Wissen Physik in der Hochschulausbildung. Konzeptualisierung, Messung, Niveaubildung ISBN 978-3-8325-3988-7 53.00 EUR

186 Marianne Korner: Cross-Age Peer Tutoring in Physik. Evaluation einer Unterrichtsmethode

ISBN 978-3-8325-3979-5 38.50 EUR

187 Simone Nakoinz: Untersuchung zur Verknüpfung submikroskopischer und makroskopischer Konzepte im Fach Chemie ISBN 978-3-8325-4057-9 38.50 EUR

188 Sandra Anus: Evaluation individueller Förderung im Chemieunterricht.Adaptivität von Lerninhalten an das Vorwissen von Lernenden am Beispiel des Basiskonzeptes Chemische Reaktion

ISBN 978-3-8325-4059-3 43.50 EUR

189 Thomas Roßbegalle: Fachdidaktische Entwicklungsforschung zum besseren Verständnis atmosphärischer Phänomene. Treibhauseffekt, saurer Regen und stratosphärischer Ozonabbau als Kontexte zur Vermittlung von Basiskonzepten der Chemie ISBN 978-3-8325-4059-3 45.50 EUR

190 Kathrin Steckenmesser-Sander: Gemeinsamkeiten und Unterschiede physikbezogener Handlungs-, Denk- und Lernprozesse von Mädchen und Jungen ISBN 978-3-8325-4066-1 38.50 EUR 
191 Cornelia Geller: Lernprozessorientierte Sequenzierung des Physikunterrichts im Zusammenhang mit Fachwissenserwerb. Eine Videostudie in Finnland, Deutschland und der Schweiz ISBN 978-3-8325-4082-1 35.50 EUR

192 Jan Hofmann: Untersuchung des Kompetenzaufbaus von Physiklehrkräften während einer Fortbildungsmaßnahme ISBN 978-3-8325-4104-0 38.50 EUR

193 Andreas Dickhäuser: Chemiespezifischer Humor. Theoriebildung, Materialentwicklung, Evaluation ISBN 978-3-8325-4108-8 37.00 EUR

194 Stefan Korte: Die Grenzen der Naturwissenschaft als Thema des Physikunterrichts ISBN 978-3-8325-4112-5 57.50 EUR

195 Carolin Hülsmann: Kurswahlmotive im Fach Chemie. Eine Studie zum Wahlverhalten und Erfolg von Schülerinnen und Schülern in der gymnasialen Oberstufe ISBN 978-3-8325-4144-6 49.00 EUR

196 Caroline Körbs: Mindeststandards im Fach Chemie am Ende der Pflichtschulzeit ISBN 978-3-8325-4148-4 34.00 EUR

197 Andreas Vorholzer: Wie lassen sich Kompetenzen des experimentellen Denkens und Arbeitens fördern? Eine empirische Untersuchung der Wirkung eines expliziten und eines impliziten Instruktionsansatzes

ISBN 978-3-8325-4194-1 37.50 EUR

198 Anna Katharina Schmitt: Entwicklung und Evaluation einer Chemielehrerfortbildung zum Kompetenzbereich Erkenntnisgewinnung

ISBN 978-3-8325-4228-3 39.50 EUR

199 Christian Maurer: Strukturierung von Lehr-Lern-Sequenzen ISBN 978-3-8325-4247-4 36.50 EUR

200 Helmut Fischler, Elke Sumfleth (Hrsg.): Professionelle Kompetenz von Lehrkräften der Chemie und Physik

ISBN 978-3-8325-4523-9 34.00 EUR

201 Simon Zander: Lehrerfortbildung zu Basismodellen und Zusammenhänge zum Fachwissen

ISBN 978-3-8325-4248-1 35.00 EUR

202 Kerstin Arndt: Experimentierkompetenz erfassen.

Analyse von Prozessen und Mustern am Beispiel von Lehramtsstudierenden der Chemie

ISBN 978-3-8325-4266-5 45.00 EUR

203 Christian Lang: Kompetenzorientierung im Rahmen experimentalchemischer Praktika ISBN 978-3-8325-4268-9 42.50 EUR

204 Eva Cauet: Testen wir relevantes Wissen? Zusammenhang zwischen dem Professionswissen von Physiklehrkräften und gutem und erfolgreichem Unterrichten

ISBN 978-3-8325-4276-4 39.50 EUR 
205 Patrick Löffler: Modellanwendung in Problemlöseaufgaben. Wie wirkt Kontext? ISBN 978-3-8325-4303-7 35.00 EUR

206 Carina Gehlen: Kompetenzstruktur naturwissenschaftlicher Erkenntnisgewinnung im Fach Chemie ISBN 978-3-8325-4318-1 43.00 EUR

207 Lars Oettinghaus: Lehrerüberzeugungen und physikbezogenes Professionswissen. Vergleich von Absolventinnen und Absolventen verschiedener Ausbildungswege im Physikreferendariat ISBN 978-3-8325-4319-8 38.50 EUR

208 Jennifer Petersen: Zum Einfluss des Merkmals Humor auf die Gesundheitsförderung im Chemieunterricht der Sekundarstufe I.

Eine Interventionsstudie zum Thema Sonnenschutz ISBN 978-3-8325-4348-8 40.00 EUR

209 Philipp Straube: Modellierung und Erfassung von Kompetenzen naturwissenschaftlicher Erkenntnisgewinnung bei (Lehramts-) Studierenden im Fach Physik ISBN 978-3-8325-4351-8 35.50 EUR

210 Martin Dickmann: Messung von Experimentierfähigkeiten. Validierungsstudien zur Qualität eines computerbasierten Testverfahrens ISBN 978-3-8325-4356-3 41.00 EUR

211 Markus Bohlmann: Science Education. Empirie, Kulturen und Mechanismen der Didaktik der Naturwissenschaften ISBN 978-3-8325-4377-8 44.00 EUR

212 Martin Draude: Die Kompetenz von Physiklehrkräften, Schwierigkeiten von Schülerinnen und Schülern beim eigenständigen Experimentieren zu diagnostizieren ISBN 978-3-8325-4382-2 37.50 EUR

213 Henning Rode: Prototypen evidenzbasierten Physikunterrichts. Zwei empirische Studien zum Einsatz von Feedback und Blackboxes in der Sekundarstufe ISBN 978-3-8325-4389-1 42.00 EUR

214 Jan-Henrik Kechel: Schülerschwierigkeiten beim eigenständigen Experimentieren. Eine qualitative Studie am Beispiel einer Experimentieraufgabe zum Hooke'schen Gesetz

ISBN 978-3-8325-4392-1 55.00 EUR

215 Katharina Fricke: Classroom Management and its Impact on Lesson Outcomes in Physics. A multi-perspective comparison of teaching practices in primary and secondary schools

ISBN 978-3-8325-4394-5 40.00 EUR

216 Hannes Sander: Orientierungen von Jugendlichen beim Urteilen und Entscheiden in Kontexten nachhaltiger Entwicklung. Eine rekonstruktive Perspektive auf Bewertungskompetenz in der Didaktik der Naturwissenschaft ISBN 978-3-8325-4434-8 46.00 EUR 
217 Inka Haak: Maßnahmen zur Unterstützung kognitiver und metakognitiver Prozesse in der Studieneingangsphase. Eine Design-Based-Research-Studie zum universitären Lernzentrum Physiktreff ISBN 978-3-8325-4437-9

$$
\text { 46.50 EUR }
$$

218 Martina Brandenburger: Was beeinflusst den Erfolg beim Problemlösen in der Physik? Eine Untersuchung mit Studierenden ISBN 978-3-8325-4409-6 42.50 EUR

219 Corinna Helms: Entwicklung und Evaluation eines Trainings zur Verbesserung der Erklärqualität von Schülerinnen und Schülern im Gruppenpuzzle ISBN 978-3-8325-4454-6 42.50 EUR

220 Viktoria Rath: Diagnostische Kompetenz von angehenden Physiklehrkräften. Modellierung, Testinstrumentenentwicklung und Erhebung der Performanz bei der Diagnose von Schülervorstellungen in der Mechanik

ISBN 978-3-8325-4456-0 42.50 EUR

221 Janne Krüger: Schülerperspektiven auf die zeitliche Entwicklung der Naturwissenschaften

ISBN 978-3-8325-4457-7 45.50 EUR

222 Stefan Mutke: Das Professionswissen von Chemiereferendarinnen und -referendaren in Nordrhein-Westfalen. Eine Längsschnittstudie ISBN 978-3-8325-4458-4 37.50 EUR

223 Sebastian Habig: Systematisch variierte Kontextaufgaben und ihr Einfluss auf kognitive und affektive Schülerfaktoren

ISBN 978-3-8325-4467-6 40.50 EUR

224 Sven Liepertz: Zusammenhang zwischen dem Professionswissen von Physiklehrkräften, dem sachstrukturellen Angebot des Unterrichts und der Schülerleistung ISBN 978-3-8325-4480-5 34.00 EUR

225 Elina Platova: Optimierung eines Laborpraktikums durch kognitive Aktivierung ISBN 978-3-8325-4481-2 39.00 EUR

226 Tim Reschke: Lesegeschichten im Chemieunterricht der Sekundarstufe I zur Unterstützung von situationalem Interesse und Lernerfolg ISBN 978-3-8325-4487-4 41.00 EUR

227 Lena Mareike Walper: Entwicklung der physikbezogenen Interessen und selbstbezogenen Kognitionen von Schülerinnen und Schülern in der Übergangsphase von der Primar- in die Sekundarstufe. Eine Längsschnittanalyse vom vierten bis zum siebten Schuljahr

ISBN 978-3-8325-4495-9 43.00 EUR

228 Stefan Anthofer: Förderung des fachspezifischen Professionswissens von Chemielehramtsstudierenden ISBN 978-3-8325-4498-0 39.50 EUR

229 Marcel Bullinger: Handlungsorientiertes Physiklernen mit instruierten Selbsterklärungen in der Primarstufe. Eine experimentelle Laborstudie ISBN 978-3-8325-4504-8 44.00 EUR 
230 Thomas Amenda: Bedeutung fachlicher Elementarisierungen für das Verständnis der Kinematik

ISBN 978-3-8325-4531-4 43.50 EUR

231 Sabrina Milke: Beeinflusst Priming das Physiklernen?

Eine empirische Studie zum Dritten Newtonschen Axiom

ISBN 978-3-8325-4549-4 42.00 EUR

232 Corinna Erfmann: Ein anschaulicher Weg zum Verständnis der elektromagnetischen Induktion. Evaluation eines Unterrichtsvorschlags und Validierung eines Leistungsdiagnoseinstruments

ISBN 978-3-8325-4550-5 49.50 EUR

233 Hanne Rautenstrauch: Erhebung des (Fach-)Sprachstandes bei Lehramtsstudierenden im Kontext des Faches Chemie

ISBN 978-3-8325-4556-7 40.50 EUR

234 Tobias Klug: Wirkung kontextorientierter physikalischer Praktikumsversuche auf Lernprozesse von Studierenden der Medizin

ISBN 978-3-8325-4558-1 37.00 EUR

235 Mareike Bohrmann: Zur Förderung des Verständnisses der Variablenkontrolle im naturwissenschaftlichen Sachunterricht

ISBN 978-3-8325-4559-8 52.00 EUR

236 Anja Schödl: FALKO-Physik - Fachspezifische Lehrerkompetenzen im Fach Physik. Entwicklung und Validierung eines Testinstruments zur Erfassung des fachspezifischen Professionswissens von Physiklehrkräften ISBN 978-3-8325-4553-6 40.50 EUR

237 Hilda Scheuermann: Entwicklung und Evaluation von Unterstützungsmaßnahmen zur Förderung der Variablenkontrollstrategie beim Planen von Experimenten ISBN 978-3-8325-4568-0 39.00 EUR

238 Christian G. Strippel: Naturwissenschaftliche Erkenntnisgewinnung an chemischen Inhalten vermitteln. Konzeption und empirische Untersuchung einer Ausstellung mit Experimentierstation

ISBN 978-3-8325-4577-2 41.50 EUR

239 Sarah Rau: Durchführung von Sachunterricht im Vorbereitungsdienst. Eine längsschnittliche, videobasierte Unterrichtsanalyse

ISBN 978-3-8325-4579-6 46.00 EUR

240 Thomas Plotz: Lernprozesse zu nicht-sichtbarer Strahlung. Empirische Untersuchungen in der Sekundarstufe 2

ISBN 978-3-8325-4624-3 39.50 EUR 
241 Wolfgang Aschauer: Elektrische und magnetische Felder. Eine empirische Studie zu Lernprozessen in der Sekundarstufe II

ISBN 978-3-8325-4625-0 50.00 EUR

242 Anna Donhauser: Didaktisch rekonstruierte Materialwissenschaft. Aufbau und Konzeption eines Schülerlabors für den Exzellenzcluster Engineering of Advanced Materials

ISBN 978-3-8325-4636-6 39.00 EUR

243 Katrin Schüßler: Lernen mit Lösungsbeispielen im Chemieunterricht. Einflüsse auf Lernerfolg, kognitive Belastung und Motivation

ISBN 978-3-8325-4640-3 42.50 EUR

244 Timo Fleischer: Untersuchung der chemischen Fachsprache unter besonderer Berücksichtigung chemischer Repräsentationen

ISBN 978-3-8325-4642-7 46.50 EUR

245 Rosina Steininger: Concept Cartoons als Stimuli für Kleingruppendiskussionen im Chemieunterricht. Beschreibung und Analyse einer komplexen Lerngelegenheit ISBN 978-3-8325-4647-2 39.00 EUR

246 Daniel Rehfeldt: Erfassung der Lehrqualität naturwissenschaftlicher Experimentalpraktika

ISBN 978-3-8325-4590-1 40.00 EUR

247 Sandra Puddu: Implementing Inquiry-based Learning in a Diverse Classroom: Investigating Strategies of Scaffolding and Students' Views of Scientific Inquiry ISBN 978-3-8325-4591-8 35.50 EUR

248 Markus Bliersbach: Kreativität in der Chemie. Erhebung und Förderung der Vorstellungen von Chemielehramtsstudierenden ISBN 978-3-8325-4593-2 44.00 EUR

249 Lennart Kimpel: Aufgaben in der Allgemeinen Chemie. Zum Zusammenspiel von chemischem Verständnis und Rechenfähigkeit

ISBN 978-3-8325-4618-2 36.00 EUR

250 Louise Bindel: Effects of integrated learning: explicating a mathematical concept in inquiry-based science camps

ISBN 978-3-8325-4655-7 37.50 EUR

251 Michael Wenzel: Computereinsatz in Schule und Schülerlabor. Einstellung von Physiklehrkräften zu Neuen Medien

ISBN 978-3-8325-4659-5 38.50 EUR

252 Laura Muth: Einfluss der Auswertephase von Experimenten im Physikunterricht. Ergebnisse einer Interventionsstudie zum Zuwachs von Fachwissen und experimenteller Kompetenz von Schülerinnen und Schülern

ISBN 978-3-8325-4675-5 36.50 EUR 
253 Annika Fricke: Interaktive Skripte im Physikalischen Praktikum. Entwicklung und Evaluation von Hypermedien für die Nebenfachausbildung

ISBN 978-3-8325-4676-2 41.00 EUR

254 Julia Haase: Selbstbestimmtes Lernen im naturwissenschaftlichen Sachunterricht. Eine empirische Interventionsstudie mit Fokus auf Feedback und Kompetenzerleben ISBN 978-3-8325-4685-4 38.50 EUR

255 Antje J. Heine: Was ist Theoretische Physik? Eine wissenschaftstheoretische Betrachtung und Rekonstruktion von Vorstellungen von Studierenden und Dozenten über das Wesen der Theoretischen Physik

ISBN 978-3-8325-4691-5 46.50 EUR

256 Claudia Meinhardt: Entwicklung und Validierung eines Testinstruments zu Selbstwirksamkeitserwartungen von (angehenden) Physiklehrkräften in physikdidaktischen Handlungsfeldern

ISBN 978-3-8325-4712-7 47.00 EUR

257 Ann-Kathrin Schlüter: Professionalisierung angehender Chemielehrkräfte für einen Gemeinsamen Unterricht

ISBN 978-3-8325-4713-4 53.50 EUR

258 Stefan Richtberg: Elektronenbahnen in Feldern. Konzeption und Evaluation einer webbasierten Lernumgebung

ISBN 978-3-8325-4723-3 49.00 EUR

259 Jan-Philipp Burde: Konzeption und Evaluation eines Unterrichtskonzepts zu einfachen Stromkreisen auf Basis des Elektronengasmodells

ISBN 978-3-8325-4726-4 57.50 EUR

260 Frank Finkenberg: Flipped Classroom im Physikunterricht ISBN 978-3-8325-4737-4 42.50 EUR

261 Florian Treisch: Die Entwicklung der Professionellen Unterrichtswahrnehmung im Lehr-Lern-Labor Seminar ISBN 978-3-8325-4741-4 41.50 EUR

262 Desiree Mayr: Strukturiertheit des experimentellen naturwissenschaftlichen Problemlöseprozesses

ISBN 978-3-8325-4757-8 37.00 EUR

263 Katrin Weber: Entwicklung und Validierung einer Learning Progression für das Konzept der chemischen Reaktion in der Sekundarstufe I

ISBN 978-3-8325-4762-2 48.50 EUR

Alle erschienenen Bücher können unter der angegebenen ISBN direkt online (http://www.logosverlag.de) oder per Fax (030 - 428510 92) beim Logos Verlag Berlin bestellt werden. 



\section{Studien zum Physik- und Chemielernen}

Herausgegeben von Hans Niedderer, Helmut Fischler und Elke Sumfleth

Die Reihe umfasst inzwischen eine große Zahl von wissenschaftlichen Arbeiten aus vielen Arbeitsgruppen der Physik- und Chemiedidaktik und zeichnet damit ein gültiges Bild der empirischen physik- und chemiedidaktischen Forschung in Deutschland.

Die Herausgeber laden daher Interessenten zu neuen Beiträgen ein und bitten sie, sich im Bedarfsfall an den Logos-Verlag oder an ein Mitglied des Herausgeberteams zu wenden.

\section{Kontaktadressen:}

Prof. Dr. Hans Niedderer

Institut für Didaktik der Naturwissenschaften,

Abt. Physikdidaktik, FB Physik/Elektrotechnik,

Universität Bremen,

Postfach 3304 40, 28334 Bremen

Tel. 0421-218 2484/4695, e-mail:

niedderer@physik.uni-bremen.de

Prof. Dr. Helmut Fischler

Didaktik der Physik, FB Physik, Freie Universität Berlin,

Arnimallee 14, 14195 Berlin

Tel. 030-838 56712/55966, e-mail:

fischler@physik.fu-berlin.de

Prof. Dr. Elke Sumfleth

Didaktik der Chemie,

Fachbereich Chemie,

Universität Duisburg-Essen,

Schützenbahn 70, 45127 Essen

Tel. 0201-183 3757/3761, e-mail:

elke.sumfleth@uni-essen.de 
Flipped Classroom ist eine innovative Unterrichtsmethode, bei der sich Schülerinnen und Schüler zuhause mit Lernvideos auf den Unterricht vorbereiten. Im Unterricht selbst steht dann die Anwendung und Vertiefung des Wissens in kooperativen Lernsettings im Vordergrund. Wissenschaftlich ist der Einsatz dieser Methode im deutschen Schulunterricht bislang nicht untersucht worden, auch nicht im Physikunterricht. Haben die Schülerinnen und Schüler hier einen höheren Lernzuwachs? Ändern sich das Selbstkonzept, das Fachinteresse und die Motivation? Welche Rolle spielt dabei das Leistungsvermögen oder das Geschlecht?

Die vorliegende quasi-experimentelle Interventionsstudie im Pre-/Postdesign mit Kontrollgruppe untersuchte den Einsatz des Flipped Classroom im Physikunterricht. Dabei zeigten sich positive Effekte auf den Lernerfolg sowie teilweise auf affektive Lernmerkmale. Motivational profitierten vor allem die Mädchen vom Flipped Classroom, die Jungen hingegen verbesserten deutlich ihre Hausaufgabendisziplin.

\section{Logos Verlag Berlin}

ISBN 978-3-8325-4737-0 